\title{
Labelling Policies for Food
}

\author{
Dissertation \\ zur Erlangung des Doktorgrades \\ der Fakultät für Agrarwissenschaften \\ der Georg-August-Universität Göttingen
}

vorgelegt von

Ramona Weinrich

geboren in Kassel

Göttingen, im März 2015 
D 7

1. Referent:

Prof. Dr. Achim Spiller

2. Korreferent:

PD Dr. Gunnar Breustedt

Tag der mündlichen Prüfung:

21. Mai 2015 


\section{Inhaltsverzeichnis}

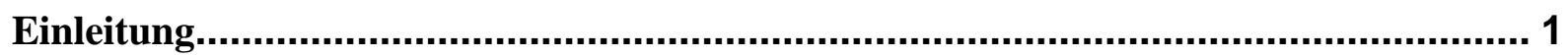

Teil I: Potentials for multi-level food labelling systems ........................................ 16

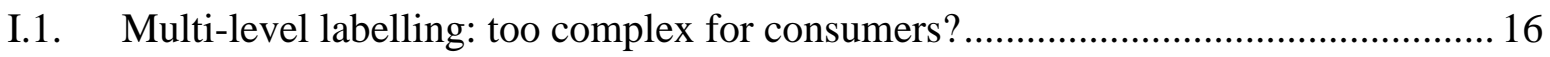

I.2 Developing food labelling strategies: multi-level labelling ................................. 17

I.3 Zahlungsbereitschaftsanalysen in mehrstufigen Labelsystemen. Das Beispiel

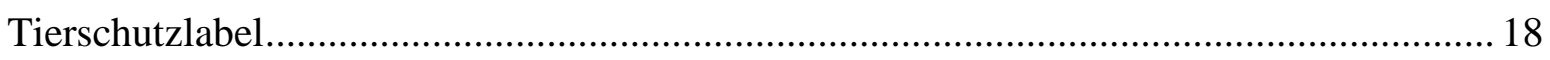

Teil II: Consumer understanding of food packaging.......................................... 44

II.1 Verbraucherverständnis von Verkehrsbezeichnungen ....................................... 44

II.2 Verbraucherverständnis der Gestaltung von Lebensmittelverpackungen ................. 66

II.3 Zum Verbraucherverständnis von Alpen- und Weidemilch..................................... 88

Teil III: Marketing strategies for niche products ............................................. 114

III.1 Consumer attitudes in Germany towards different dairy housing systems and their implications for the marketing of pasture-raised milk................................................. 114

III.2 Consumer preferences for high welfare meat in Germany: self-service counter or

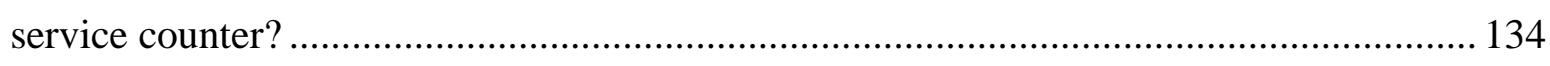

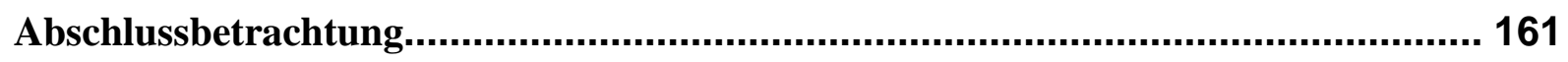

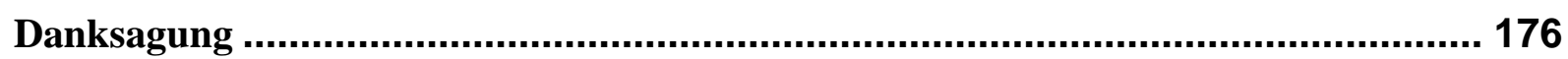

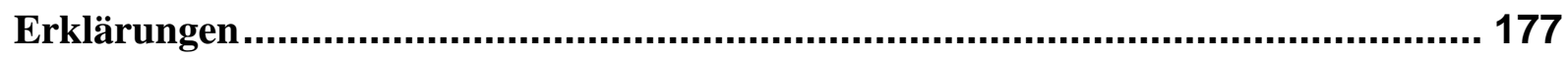





\section{Einleitung}

Das Interesse der Verbraucher ${ }^{1}$ an Lebensmitteln mit besonderen Qualitätseigenschaften nimmt zu. Bedingt durch verschiedene Lebensmittelskandale stellt Qualität ein wichtiges Einkaufsmotiv dar (BVE 2011, Caswell und Joseph 2008, Grunert 2005, Zühlsdorf et al. 2013). $\mathrm{Zu}$ den bekanntesten Lebensmittelskandalen zählen die BSE-Krise, der Nachweis von Nitrofen in Bio-Futtermitteln, Gammelfleischskandale, die EHEC-Krise oder der PferdefleischSkandal. Zuletzt wurde im Januar 2015 bekannt, dass Putenfleisch aus Discountern zu $88 \%$ mit antibiotikaresistenten Keimen belastet ist (BUND 2015). Verstärkt wird die Debatte um die Lebensmittelqualität aktuell durch das geplante Freihandelsabkommen TTIP (Transatlantic Trade and Investment Partnership). Zum Symbol der Verbraucherängste ist das „Chlorhühnchen“ geworden. Zwar ist in der EU Chlordioxid zur Fleischdesinfektion verboten, jedoch befürchten Verbraucher, dass aus den USA mit Chlordioxid behandeltes Geflügelfleisch auf dem deutschen Markt eingeführt wird.

Vor diesem Hintergrund rückt die Lebensmittelkennzeichnung stärker in den Fokus der Öffentlichkeit. Diese ist für Verbraucher ein wichtiges Informationsinstrument beim Lebensmitteleinkauf. Informationen auf der Verpackung geben Verbrauchern Aufschluss über Produktund Prozesscharakteristika des Verpackungsinhalts. So können europäische Verbraucher die Herkunft von Fleisch anhand der Kennzeichnung erkennen (Art. 26 Abs. 2 lit. b LMIV). Die Wichtigkeit der Lebensmittelkennzeichnung äußert sich nicht zuletzt durch die am 13. Dezember 2014 in Kraft getretene Lebensmittel-Informationsverordnung (LMIV), die die bis dahin in Deutschland geltende Lebensmittel-Kennzeichnungsverordnung ersetzt. Neuerungen betreffen z. B. eine verpflichtende Herkunftskennzeichnung von unverarbeitetem Schweine-, Ziegen-, Schaf- und Geflügelfleisch, eine Mindestschriftgröße auf Verpackungen oder die Kennzeichnung von Lebensmittelimitaten (Verordnung EU Nr. 1169/2011). Die Verordnung soll umfassende Verbraucherinformationen und europaweit einheitliche Herstellervorgaben gewährleisten (BMEL o. J.).

Generell können Lebensmittelkennzeichnungen in gesetzlich verpflichtende und freiwillige Angaben auf der Lebensmittelverpackung untergliedert werden. $\mathrm{Zu}$ den verpflichtenden Angaben zählen u. a. (Art. 9 Abs. 1 LMIV):

- die Bezeichnung des Lebensmittels,

\footnotetext{
${ }^{1}$ Aus Gründen der Vereinfachung wird ausschließlich die männliche Form verwendet. Personen weiblichen wie männlichen Geschlechts sind darin gleichermaßen eingeschlossen.
} 
- das Zutatenverzeichnis,

- potenziell Allergien und Unverträglichkeiten auslösende Zutaten und Verarbeitungshilfsstoffe,

- die Menge bestimmter Zutaten, wenn diese z. B. auf der Verpackung grafisch oder verbal hervorgehoben werden oder wesentlich für die Charakterisierung des Lebensmittels sind,

- die Nettofüllmenge des Lebensmittels,

- das Mindesthaltbarkeits- oder Verbrauchsdatum,

- das Ursprungsland oder der Herkunftsort bei bestimmten Lebensmitteln,

- eine Nährwertdeklaration.

Die Informationen auf Lebensmittelverpackungen sind für Verbraucher von besonderer Wichtigkeit, da Konsumenten beim Einkauf nicht alle Eigenschaften am Produkt vor Ort überprüfen können. Generell haben Hersteller und Produzenten mehr Informationen über ihre Produkte als Verbraucher, wodurch Informationsasymmetrien hervorgerufen werden. Bei Vertrauenseigenschaften ist die Informationsasymmetrie besonders stark ausgeprägt, da diese i. d. R. weder vor noch nach dem Kauf bzw. Verzehr von Verbrauchern überprüft werden können (Darby und Karni 1973, Becker 2000). Ein Beispiel hierfür ist der Eiweißanteil im Fleisch, der nur durch eine Laboranalyse ermittelt werden kann. Darüber hinaus gibt es Eigenschaften, die am Endprodukt auch von Experten nicht analysiert werden können. Beim Fleisch ist dies etwa die Haltungsform der Tiere. Diese nicht am Produkt nachweisbaren Eigenschaften werden in der Literatur als Potemkin-Eigenschaften bezeichnet (Tietzel und Weber 1991$)^{2}$. Da jedoch i. d. R. weder Vertrauens- noch Potemkin-Eigenschaften von Verbrauchern überprüft werden können, wird zur Vereinfachung im Folgenden nur von Vertrauenseigenschaften gesprochen.

Daher werden verschiedene Kennzeichnungselemente eingesetzt, um Informationsasymmetrien abzubauen und Verbrauchern Prozess- und / oder Produktqualitäten als sog. Sucheigenschaft zu kommunizieren (Antle 2001, Darby und Karni 1973, Nelson 1970, Caswell und Mojduszka 1996, Caswell und Padberg 1992, Jahn et al. 2005). Bspw. kann ein Label signalisieren, dass Fleisch besonders tierfreundlich produziert wurde. Sucheigenschaften zeichnen sich dadurch aus, dass Verbraucher sie selbst am Lebensmittel und direkt beim Einkaufen überprüfen können. Ein weiteres Beispiel neben Labeln ist die Marmorierung eines Fleisch-

\footnotetext{
${ }^{2}$ Den Begriff Potemkin-Eigenschaft leiten Tietzel und Weber (1991) von folgendem Ereignis ab: Der politische Ratgeber und Günstling Grigori Alexandrowitsch Potjomkin der russischen Zarin Katharina II. soll Dörfer auf der neu eroberten Krim-Halbinsel zum Schein errichtet haben, um einen besseren Zustand der Gegend vorzutäuschen.
} 
stückes. Hingegen sind Erfahrungseigenschaften, z. B. die Menge Wasser, die ein Fleischstück beim Braten verliert, für Verbraucher nach dem Kauf überprüfbar (Nelson 1970, 1974). Abbildung 1 gibt die Gütereigenschaften in Zusammenhang mit der Informationsasymmetrie wieder.

Abbildung 1: Gütertypologie nach Grad der Informationsasymmetrie

\begin{tabular}{|cccc|}
\hline $\begin{array}{c}\text { Such- } \\
\text { eigenschaften }\end{array}$ & $\begin{array}{c}\text { Erfahrungs- } \\
\text { eigenschaften }\end{array}$ & $\begin{array}{c}\text { Vertrauens- } \\
\text { eigenschaften }\end{array}$ & $\begin{array}{c}\text { Potemkin- } \\
\text { eigenschaften }\end{array}$ \\
\hline & Zunahme der Informationsasymmetrie & \\
\hline
\end{tabular}

Quelle: eigene Darstellung in Anlehnung an Tietzel und Weber (1991)

In der vorliegenden Dissertation liegt der Schwerpunkt auf der Lebensmittelkennzeichnung von Vertrauenseigenschaften. Für Verbraucher besonders wichtige Eigenschaften sind nach Fernqvist und Ekelund (2014) gesundheitsbezogene Angaben, ökologische Produktionsweise, Herkunftsangaben, ethische Angaben wie z. B. Fair Trade oder Tierschutz und beschreibende Lebensmittelnamen. Hersteller bzw. Produzenten heben durch Kennzeichnungselemente auf Verpackungen solche Produktbesonderheiten hervor. Diese Angaben sind z. T. freiwillig und können folgendermaßen untergliedert werden:

- Nährwert- und gesundheitsbezogene Angaben wie z. B.:

$\checkmark$,Vitamin C erhöht die Eisenaufnahme“

$\checkmark$,enthält viel Vitamin C“

- Freiwillige Label, die auf gesetzlichen Regeln beruhen, wie z. B.:

$\checkmark$ Staatliches Bio-Siegel

$\checkmark$ EU-Gütesiegel (z B. „Geschützte Ursprungsbezeichnung“, „Geschützte geografische Angabe“, „Garantiert traditionelle Spezialität“)

$\checkmark$,Ohne-Gentechnik“-Siegel

- Freiwillige, privatwirtschaftlich getragene Label wie z. B.:

$\checkmark$ Bio-Siegel der Anbauverbände (bspw. Demeter, Bioland oder Naturland)

$\checkmark$ Label, die durch unabhängige Stellen mitentwickelt und vergeben werden (z. B. das Marine Stewardship Council (MSC)-Siegel oder das Fair-TradeSiegel)

Die freiwilligen und verpflichtenden Angaben sind jeweils geeignet, Unterschiede bei Produktqualitäten hervorzuheben. Gelingt dies durch die Kennzeichnung nicht, können Verbraucher Unterschiede in der Qualität nicht erkennen. Folglich werden Verbraucher für qualitativ 
höherwertige Produkte in diesem Fall keine höheren Zahlungsbereitschaften haben. Dann würden bessere Qualitäten vom Markt verschwinden, da für diese kein Mehrpreis erzielt werden kann. Die Folge kann langfristig Marktversagen sein (Akerlof 1970). Ein Mehrpreis wird von Verbrauchern nur dann gezahlt, wenn sie eine höhere Qualität erkennen (McCluskey und Leiro 2003). Daher ist es von essenzieller Bedeutung, dass die Lebensmittelkennzeichnung transparent ist und unterschiedliche Qualitäten an Hand der Lebensmittelverpackung für Verbraucher kenntlich werden.

Die Literatur zeigt jedoch auf, dass die derzeit gängige Lebensmittelkennzeichnung auf Verpackungen Schwächen aufweist. So konstatieren Annunziata et al. (2011), dass zwar nur 7 \% der Teilnehmer ihrer Studie Nachhaltigkeits-Label auf Verpackungen gar nicht beachten. Die Verbraucher, die die Kennzeichnung beachten, sind aber mit dieser oftmals nicht zufrieden. Die Studienteilnehmer berichten von Schwierigkeiten beim Verstehen der Informationen auf Verpackungen und von mangelndem Vertrauen in zugrunde liegende Produktstandards. Eine weltweite Studie mit 21.000 Verbrauchern legt zudem offen, dass Lebensmittelverpackungen für viele Verbraucher unverständlich sind: Etwa $90 \%$ der Verbraucher lesen Informationen auf Lebensmittelverpackungen, aber mehr als die Hälfte verstehen die Angaben auf den Verpackungen nicht (ACNielsen 2005). Weiterhin empfinden viele Verbraucher die zunehmende Anzahl an Labeln als verwirrend (Brécard 2014, European Commission 2009, Harbaugh et al. 2011), obwohl diese eine Einkaufsorientierung und -erleichterung darstellen sollen (Verbeke 2005a).

Die Ursachen für die unzureichende Lebensmittelkennzeichnung sind vielfältig. Die von der Marketingforschung seit längerem konstatierte Informationsüberlastung (information overload) ist ein Erklärungsfaktor (Kolodinsky 2012, Kroeber-Riel und Esch 2004, van Kleef et al. 2008). Die Informationsüberlastung ist dadurch gekennzeichnet, dass Verbraucher sich in der heutigen Zeit mit einer Vielzahl an Informationen konfrontiert sehen. Die Aufnahme von Informationen auf Lebensmittelverpackungen ist aber durch die Aufnahmekapazität, die Verarbeitungsfähigkeit (Verbeke 2005b) und die Fähigkeit, die Informationen zu verstehen, limitiert (Sørensen et al. 2012). Eine Studie von Chen et al. (2009) bestätigt, dass die Informationsüberlastung bei Konsumenten mit der Zunahme der Informationsbereitstellung stärker wird. Demzufolge nimmt die Informationsverarbeitung mit der Menge an Informationen ab. Daher ist es für Verbraucher schwierig, die entscheidenden Informationen auf Lebensmittelverpackungen auszuwählen und zu verarbeiten (Jacoby et al. 1974). 
Neben der Informationsüberlastung sind Verbraucher zudem mit einer Auswahlüberlastung (choice overload) konfrontiert. Das führt dazu, dass Verbraucher bei zu viel Auswahl keine rationale (Jacoby et al. 1974) oder eine falsche Entscheidung bei der Produktwahl treffen (Malhotra 1982). Verschärft wird die Informations- und Auswahlüberlastung durch die abnehmende Zeit, die Verbraucher für den Lebensmitteinkauf aufwenden (Caswell und Padberg 1992, Procher und Vance 2013). Verbraucherinformationen auf der Verpackungsvorderseite werden daher nur kurz betrachtet, was die Informationsaufnahme und -verarbeitung zusätzlich limitiert (Königstorfer und Gröppel-Klein 2012).

Ausgelobte Prozess- und Produkteigenschaften sollten wegen der aufgezeigten Probleme transparent und in vereinfachter Form kommuniziert werden. Verbrauchern muss es möglich sein, durch Schlüsselinformationen auf Verpackungen die Produktbeschaffenheit und die zu Grunde liegenden Prozesseigenschaften zu erkennen. Vor diesem Hintergrund wird im Folgenden die Dissertation in den aktuellen Stand der Forschung eingeordnet und der Forschungsbedarf wird aufgezeigt.

\section{Einordnung der Dissertation in den Forschungsbereich}

In der Konsumentenforschung bisher weitgehend unbeachtet sind mehrstufige Labelsysteme, die unterschiedliche Standards von Prozesseigenschaften kommunizieren. Wissenschaftliche Veröffentlichungen zu Kennzeichnungssystemen analysieren schwerpunktmäßig die Wirkung von komplexen Nährstoffkennzeichnungen im Vergleich zu vereinfachten Labelsystemen (Andrews et al. 2007, Feunekes et al. 2008, Grunert und Wills 2007, Kelly et al. 2009). Erste Studien von Fischer and Lyon (2012, 2013) weisen Potenzial für ein mehrstufiges Kennzeichnungssystem für Ökolabel auf. Nach eingehender Literaturrecherche sind keine Arbeiten in der Konsumentenforschung veröffentlicht, die Auswirkungen mehrstufiger Labelsysteme untersuchen, die unterschiedliche Prozessstandards in der Lebensmittelherstellung kommunizieren.

Auf Grund der beschriebenen Informationsüberlastung ist es für Verbraucher von großer Wichtigkeit, sich auf Schlüsselinformationen verlassen zu können, um die Beschaffenheit und damit die Produktzusammensetzung beurteilen zu können. Bisherige Veröffentlichungen beschäftigen sich schwerpunktmäßig mit binären Food Labeln (z. B. Harper et al. 2007). Health Claims (z. B. de la Hunty et al. 2014) und die Nährwertkennzeichnung auf Lebensmittelverpackungen (z. B. Bialkova und van Trijp 2010) sind weitere Schwerpunkte in Veröffentlichungen. Das Verbraucherverständnis von Kennzeichnungselementen wie Verkehrsbezeich- 
nung, Produktname und Produkt- bzw. Zutatenabbildung sowie Clean Labeln ist bisher aus wissenschaftlicher Sicht empirisch nicht untersucht worden.

Neben der Kennzeichnung von Lebensmitteln ist es ebenso wichtig, dass das Marketing von Produkten bestimmter Prozesseigenschaften an die jeweiligen Zielgruppen angepasst wird. Für verschiedene Nischenprodukte wie Tierschutzfleisch und Weidemilch, denen ethische Vertrauenseigenschaften zu Grunde liegen, sind bereits Marktpotenziale aufgedeckt worden (Elbakadize et al. 2013, Ellis et al. 2009, Harper und Makatouni 2002, Hellberg-Bahr et al. 2012, Kehlbacher et al. 2012, Lagerkvist und Hess 2011, Pirog 2004, Schulze et al. 2008). Bisher sind Zielgruppen für Weidemilch nicht umfassend charakterisiert worden. Zudem gibt es keine Studien dazu, wo Tierschutzfleisch im Lebensmitteleinzelhandel am erfolgversprechendsten zu platzieren ist. Die Analyse dieser Forschungslücken kann einen Beitrag zur optimierten Vermarktung von Nischenprodukten leisten.

Die vorliegende Dissertation ist der Konsumentenforschung zuzuordnen und findet Anwendung im Marketing und in der Verbraucherpolitik. Die fokussierten Forschungsthemen sind anwendungsorientiert und haben das Ziel, einen Wissenstransfer in die Praxis zu leisten.

\section{Ziele und Aufbau der Dissertation}

Die vorliegende Dissertation hat das Ziel, verschiedene Labelling Policies ${ }^{3}$ mittels empirischer Konsumentenbefragungen $\mathrm{zu}$ untersuchen, zu bewerten und Empfehlungen für Entscheidungsträger in der Lebensmittelkennzeichnung abzuleiten. Die Ergebnisse der Arbeit lassen Rückschlüsse darauf zu, ob bzw. welche Defizite die derzeitige Lebensmittelkennzeichnungspraxis aufweist. Darüber hinaus werden Empfehlungen gegeben, wie die Kennzeichnungspraxis verbessert werden kann. Die Erkenntnisse werden durch Marketingstrategien für Nischenprodukte erweitert und vertieft.

Die Dissertation untergliedert sich in die drei oben beschriebenen Forschungsfelder, die sich mit unterschiedlichen Aspekten von Labelling Policies beschäftigen. In den drei Kapiteln soll auf folgende Forschungsfragen eingegangen werden:

- Wie können Prozess- und Produkteigenschaften differenziert auf Lebensmittelverpackungen kommuniziert werden? (Teil I: Potentials for multi-level food labelling systems)

\footnotetext{
${ }^{3}$ Mit Labelling Policies sind in dieser Arbeit Prinzipien und / oder Vorgehensweisen in der Lebensmittelkennzeichnung gemeint, die von Organisationen oder Individuen übernommen sind oder vorgeschlagen werden (vgl. Oxford Dictionary 2015). Labelling Policies sind wichtige Konzepte, um Konsumenten entscheidende Informationen eines Lebensmittels anhand von Kennzeichnungselementen zu vermitteln (vgl. Wies 1994).
} 
- Wie werden verschiedene Kennzeichnungselemente auf Produktverpackungen, die Schlüsselinformationen für Verbraucher darstellen, von Verbrauchern wahrgenommen und beurteilt? (Teil II: Consumer understanding of food packaging)

- Welches sind für die Nischenprodukte Tierschutzfleisch und Weidemilch geeignete Marketingstrategien und durch welche Merkmale lassen sich Zielgruppen charakterisieren? (Teil III: Marketing strategies for niche products)

Der Aufbau der Dissertation ist in Tabelle 1 beschrieben. Der Darstellung ist zu entnehmen, welche Artikel dem jeweiligen Kapitel zugeordnet sind. Ergänzend werden die Autoren der Artikel angegeben und Informationen zur Veröffentlichung bzw. Einreichung der Artikel geliefert. 
Tabelle 1: Aufbau der Dissertation

\begin{tabular}{|c|c|c|}
\hline \multicolumn{3}{|c|}{ Einleitung } \\
\hline \multicolumn{3}{|c|}{ Potentials for multi-level food labelling systems } \\
\hline I. 1 & $\begin{array}{l}\text { Multi-level labelling: too complex } \\
\text { for consumers? }\end{array}$ & $\begin{array}{l}\text { R. Weinrich, A. Franz, A. Spiller } \\
\text { Eingereicht bei Economia Agro-Alimentare }\end{array}$ \\
\hline I. 2 & $\begin{array}{l}\text { Developing food labelling strate- } \\
\text { gies with the help of extremeness } \\
\text { aversion }\end{array}$ & $\begin{array}{l}\text { R. Weinrich, A. Spiller } \\
\text { Eingereicht beim Journal of Cleaner } \\
\text { Production }\end{array}$ \\
\hline I.3 & $\begin{array}{l}\text { Zahlungsbereitschaftsanalysen in } \\
\text { mehrstufigen Labelsystemen. Das } \\
\text { Beispiel Tierschutzlabel }\end{array}$ & $\begin{array}{l}\text { R. Weinrich, A. Franz, A. Spiller } \\
\text { Berichte über Landwirtschaft, 2014: } 92 \text { (2), 1- } \\
19\end{array}$ \\
\hline \multicolumn{3}{|c|}{ Consumer understanding of food packaging } \\
\hline II.1 & $\begin{array}{l}\text { Verbraucherverständnis von } \\
\text { Verkehrsbezeichnungen }\end{array}$ & $\begin{array}{l}\text { R. Weinrich, S. Nitzko, A. Spiller, A. Zühls- } \\
\text { dorf } \\
\text { Journal für Verbraucherschutz und Lebens- } \\
\text { mittelsicherheit, 2015: } 10(1), 13-22\end{array}$ \\
\hline II. 2 & $\begin{array}{l}\text { Verbraucherverständnis der Ge- } \\
\text { staltung von Lebensmittelverpa- } \\
\text { ckungen }\end{array}$ & $\begin{array}{l}\text { R. Weinrich, S. Nitzko, A. Spiller, A. Zühls- } \\
\text { dorf } \\
\text { Schriften der Gesellschaft für Wirtschafts- } \\
\text { und Sozialwissenschaften des Landbaues e.V. } \\
\text { "Neuere Theorien und Methoden in den Wirt- } \\
\text { schafts- und Sozialwissenschaften des Land- } \\
\text { baus", 2015: 50, 235-248 }\end{array}$ \\
\hline II. 3 & $\begin{array}{l}\text { Zum Verbraucherverständnis von } \\
\text { Alpen- und Weidemilch }\end{array}$ & $\begin{array}{l}\text { R. Weinrich, S. Kühl, A. Zühlsdorf, A. Spiller } \\
\text { Angenommen zur Veröffentlichung beim } \\
\text { German Journal of Agricultural Economics }\end{array}$ \\
\hline \multicolumn{3}{|c|}{ Marketing strategies for niche products } \\
\hline III.1 & $\begin{array}{l}\text { Consumer attitudes in Germany } \\
\text { towards different dairy housing } \\
\text { systems and their implications for } \\
\text { the marketing of pasture-raised } \\
\text { milk }\end{array}$ & $\begin{array}{l}\text { R. Weinrich, S. Kühl,, A. Zühlsdorf, A. Spil- } \\
\text { ler } \\
\text { International Food and Agribusiness Man- } \\
\text { agement Review, 2014: } 17 \text { (4), 205-222 }\end{array}$ \\
\hline III. 2 & $\begin{array}{l}\text { Consumer preferences for meat in } \\
\text { Germany: self-service counter or } \\
\text { service counter? }\end{array}$ & $\begin{array}{l}\text { R. Weinrich, S. Kühl, A. Franz, A. Spiller } \\
\text { International Journal on Food System Dynam- } \\
\text { ics, 2015: } 6 \text { (1), 32-49 }\end{array}$ \\
\hline \multicolumn{3}{|c|}{ Abschlussbetrachtung } \\
\hline
\end{tabular}

Im Folgenden werden die einzelnen Artikel der Dissertation vorgestellt und in den Kontext des jeweiligen Kapitels gesetzt. Zusätzlich werden die jeweiligen Ziele der Artikel vertieft 
und die verwendeten Methoden dargestellt. Anschließend folgen die jeweiligen Forschungsartikel in der Form, wie sie im jeweiligen Journal bzw. Tagungsband veröffentlich bzw. eingereicht sind. Die Dissertation endet mit Schlussfolgerungen, Implikationen für die Agrar- und Ernährungswirtschaft und Politikempfehlungen. Ergänzend werden Limitationen der Arbeit sowie ein Überblick über weiteren Forschungsbedarf dargestellt. Abschließend werden ein Fazit und ein Ausblick auf weitere Entwicklungen gegeben.

\section{Teil I: Potentials for multi-level food labelling systems}

Der erste Teil der Dissertation hat das Ziel, Potenziale für ein mehrstufiges Labelsystem aufzudecken. Derzeit dominieren binäre Label den Lebensmittelmarkt. Trägt bspw. ein Produkt das Bio-Label, ist es biologisch herstellt. Die Abwesenheit sagt aus, dass es nicht nach biologischen Richtlinien hergestellt ist. Ein mehrstufiges Labelsystem dagegen kann verschiedene Standards von Prozesseigenschaften in abgestufter Form kommunizieren. Ein Beispiel ist Tierschutz: Tierschutz ist wissenschaftlich betrachtet eine stetige und keine binäre Variable.

Um Erfolg bzw. die Marktdurchdringung für neu einzuführende, mehrstufige Labelsysteme zu erreichen, müssen Verbraucher die Stufigkeit des Labels erkennen und verstehen. Einerseits können Verbrauchern durch die Mehrstufigkeit unterschiedliche Standards transparent kommuniziert werden, andererseits ist das Label komplexer als ein binäres Labelsystem. Daher ist die Wirkung eines mehrstufigen Labels auf Konsumenten unklar. Hier setzt Artikel I.1 „Multi-level labelling: too complex for consumers?” an. Es werden zwei Verbraucherstudien miteinander verglichen. Die Studien unterscheiden sich durch den Informationsgrad: In der ersten Studie erhielten die Teilnehmer eine Erklärung zu einem zweistufigen Labelsystem. In der zweiten Studie wurden zu demselben Labelsystem keine Erläuterungen gegeben. Anhand von Mittelwertvergleichen wird analysiert, ob hypothetisch ermittelte Zahlungsbereitschaften Rückschlüsse darauf zulassen, ob ein mehrstufiges Labelsystem funktionieren kann.

Eine weitere Fragestellung ist, welche Veränderungen ein mehrstufiges Label am Markt hervorruft. Artikel I.2, „Developing food labelling strategies with the help of extremeness aversion“ untersucht, ob ein mehrstufiges Labelsystem im Vergleich zu einem binären Labelsystem den Marktanteil an gelabelten Produkten erhöhen kann. Dabei wird methodisch ein SplitSampling in einer Konsumentenbefragung angewandt: Die eine Hälfte der Probanden wird zu einem binären, die zweite Hälfte zu einem analogen mehrstufigen Label befragt. Mittels uni-, bi- und multivariater Analysemethoden wird herausgearbeitet, wie sich die hypothetisch berechneten Marktanteile zwischen den beiden Splits unterscheiden. 
Weiterhin ist für die Etablierung eines mehrstufigen Labels wichtig, dass dem Mehraufwand der Produktion entsprechend unterschiedliche Zahlungsbereitschaften für die jeweiligen Stufen des Labels erreicht werden. Artikel I.3 ermittelt, ob sich Zahlungsbereitschaften zweier Stufen in einem Labelsystem signifikant unterscheiden. Nur wenn die Mehrkosten, die in der Herstellung, Verarbeitung und Vermarktung durch höhere Produktions- und Prozessstandards entstehen, auch monetär durch den Verbraucher getragen werden können, ist das System für Produzenten, Verarbeiter und Einzelhändler finanziell attraktiv. In zwei Verbraucherstudien werden Zahlungsbereitschaften deutscher Verbraucher für ein zweistufiges Tierschutzlabel analysiert. Methodisch werden Unterschiede in den Zahlungsbereitschaften durch Mittelwertvergleiche berechnet.

\section{Teil II: Consumer understanding of food packaging}

Während im ersten Teil der Arbeit Label als Kennzeichnungsinstrumente analysiert werden, werden im zweiten Teil weitere wichtige Schlüsselelemente auf Lebensmittelverpackungen untersucht. Schlüsselinformationen helfen Verbrauchern, die Beschaffenheit von Produkten zu erkennen. Denn die Auswahl an Produkten im Lebensmitteleinzelhandel ist groß. Die Zeit, die Verbraucher zum Einkaufen verwenden, nimmt hingegen ab (Procher und Vance 2013). Umso wichtiger ist es, dass Verbraucher sich auf Schlüsselinformationen auf Lebensmittelverpackungen verlassen können.

Eine solche Schlüsselinformation ist die Verkehrsbezeichnung, deren Verbraucherverständnis in Artikel II.1 „Verbraucherverständnis von Verkehrsbezeichnungen“ analysiert wird. Die Verkehrsbezeichnung soll ermöglichen, die Art eines Produktes auf den ersten Blick zu identifizieren (Art. 9 Abs. 1 lit. a VO 1169/2011/EG). Welche Verkehrsbezeichnungen zu verwenden sind, ist für einige Produkte gesetzlich festgeschrieben, z. B. für Käsesorten in der Käseverordnung. In anderen Fällen kann auf die allgemeine Verkehrsauffassung Bezug genommen oder eine beschreibende Bezeichnung genutzt werden (Art. 2 Abs. 1 lit. np VO 1169/2011/EG). Beispielsweise können einige Verkehrsbezeichnungen den Leitsätzen des Deutschen Lebensmittelbuches entnommen werden. Ist keine Bezeichnung geregelt, wird diese vom Produzenten gewählt. Bei zahlreichen Lebensmitteln kommt es zwischen Herstellern und Verbrauchervertretern zu Konflikten die Frage betreffend, ob die verwendeten Bezeichnungen der Verbrauchererwartung entsprechen bzw. irreführend wirken. Bisher gibt es keine wissenschaftlichen Untersuchungen, die das Verbraucherverständnis von Verkehrsbezeichnungen analysieren. Mittels einer Verbraucherbefragung und uni- sowie bivariater Aus- 
wertungsmethoden wird untersucht, ob die derzeit gängige Kennzeichnungspraxis ausgewählter Verkehrsbezeichnungen dem Verbraucherverständnis gerecht wird.

Neben Verkehrsbezeichnungen können auch Produkt- bzw. Zutatenabbildungen in Zusammenhang mit dem Zutatenverzeichnis oder Clean Label entscheidende, prägnante Produktcharakteristika vermitteln. Artikel II.2 „Verbraucherverständnis der Gestaltung von Lebensmittelverpackungen“ befasst sich mit der Problematik, wie Verbraucher Schlüsselinformationen auf Lebensmitteln auffassen. Wissenschaftlich ist die Verbraucherauffassung dieser Schlüsselinformationen bisher nicht untersucht worden. Ist eine Abweichung von Verbraucherwahrnehmung und der tatsächlichen Beschaffenheit und Qualität eines Produktes zu erkennen, ist eine Änderung der Kennzeichnungsregulierung bzw. der Kennzeichnungspraxis von Nöten (Palma et al. 2014). Uni- und bivariate Analysemethoden dienen der Ermittlung des Verbraucherverständnisses der genannten Schlüsselinformationen.

Der letzte Artikel des zweiten Kapitels widmet sich dem Verbraucherverständnis von Produktbezeichnungen, die mit nicht beobachtbaren Prozesseigenschaften werben. Der Artikel „Zum Verbraucherverständnis von Alpen- und Weidemilch“ untersucht, wie weitreichend das Verbraucherverständnis dieser beiden ungeschützten Produktbezeichnungen ist. Gehen Verbraucher davon aus, dass Kühe bei Alpenmilch in einem bestimmten geografischen Gebiet gehalten werden? Und bedeutet Weidemilch für sie, dass die Milchkühe Auslauf auf der Weide haben und wenn ja, über welchen Zeitraum? Nur wenn die entsprechenden Verbrauchererwartungen an Produkte, die sich durch nicht beobachtbare Prozesseigenschaften auszeichnen, nicht enttäuscht werden, können sich solche Produkte auf dem Markt etablieren. Methodisch finden uni- und bivariate Analysemethoden Anwendung.

\section{Teil III: Marketing strategies for niche products}

Im dritten Kapitel der Dissertation werden exkursartig Marketingstrategien für Nischenprodukte vertieft. Neben den Forschungsfragen der beiden vorangegangenen Kapitel zur Lebensmittelkennzeichnung ist es ebenso wichtig, Produkte durch Marketingstrategien auf die Zielgruppen auszurichten, um die Produkte erfolgreich zu vermarkten.

Derzeit gibt es aktuell viele Diskussionen über Haltungssysteme bei Milchkühen. Bisher ist aber wenig bekannt, wie das Image von Stall- und Weidehaltungssystemen in einer Gegenüberstellung von Verbrauchern bewertet wird. Artikel III.1 „Consumer attitudes in Germany towards different dairy housing systems and their implications for the marketing of pastureraised milk" hat die Zielsetzung, Verbraucher entsprechend ihren Einstellungen zu gruppieren und Marketingpotenziale für Weidemilch mittels einer Zielgruppenanalyse zu bestimmen. 
Eine Faktorenanalyse reduziert die Komplexität der untersuchten Daten. Die anschließend ausgeführte Clusteranalyse hat das Ziel, unterschiedliche Verbrauchergruppen bezüglich Einstellung zu Stall- und Weidehaltungssystemen und Qualitätsaspekten bei Lebensmitteln zu ermitteln. Ergänzend werden die Cluster durch Zahlungsbereitschaften für Weidemilch charakterisiert.

Der zweite Artikel des dritten Kapitels „Consumer preferences for meat in Germany: selfservice counter or service counter?“ thematisiert die Problematik der Vermarktung von Tierschutzfleisch, das unter höheren Tierschutzstandards produziert wird als konventionelles Fleisch. Derzeit ist das Angebot an Tierschutzfleisch marginal, obwohl Marktpotenzial nachgewiesen ist (Harper und Makatouni 2002, Kehlbacher et al. 2012, Lagerkvist und Hess 2011, Schulze et al. 2008). Mittels Faktoren- und Clusteranalysen werden Verbrauchereinstellungen zu Tierschutz und Einkaufspräferenzen im Supermarkt bezüglich Bedienungstheken und Selbstbedienungstheken untersucht. Kreuztabellen analysieren, ob Tierschutzfleisch besser an der Bedienungs- oder an der Selbstbedienungstheke platziert werden sollte, um die Zielgruppe zu erreichen.

\section{Literatur}

ACNielsen (2005). The nutrition-Conscious Global Shopper - Consumer attitudes towards nutrition label on food packaging in Europe. ACNielsen UK.

Akerlof, G.A. (1970). The market for "Lemons": Qualitative uncertainty and the market mechanism. Quarterly Journal of Economics 84 (3), 488-500.

Andrews, J.C., Burton, S., Kees, J. (2011). Is Simpler Always Better? Consumer Evaluations of Front-of-Package Nutrition Symbols. Journal of Public Policy and Marketing 30 (2), 175-190.

Annunziata, A., Ianuario, S., Pascale, P. (2011). Consumers' attitudes toward labelling of ethical products: the case of organic and fair trade products. Journal of Food Products Marketing, 17 (5), 518-535.

Antle, J.M. (2001). Economic analysis of food safety. In: Gardner, B., Rausser, G. (Hrsg.), Handbook of agricultural economics, 1B Amsterdam, S. 1084-1136.

Becker, T. (2000). Consumer perception of fresh meat quality: a framework for analysis. British Food Journal 102 (3), 158-176.

Bialkova, S., van Trijp, H. (2010). What determines consumer attention to nutrition labels? Food Quality and Preference 21 (8), 1042-1051.

BMEL (o. J.). EU-weit einheitliche Lebensmittelkennzeichnung. URL: http://www. bmel.de/DE/Ernaehrung/Kennzeichnung/_Texte/DossierKennzeichnung.html?docId=43 1912. Abrufdatum: 08.01.2015. 
Brécard, D. (2014). Consumer confusion over the profusion of eco-labels: Lessons from a double differentiation model. Resource and Energy Economics 37: 64-84.

BUND (2015). Putenfleisch aus Discountern mit antibiotikaresistenten Keimen belastet. URL: http://www.bund.net/nc/presse/pressemitteilungen/detail/artikel/putenfleisch-ausdiscountern-mit-krankheitskeimen-belastet-risiken-und-nebenwirkungen-der-industrie/. Abrufdatum: 27.02.2015.

BVE (Hrsg.) (2011). Consumers' Choice '11 - Lebensmittelqualität im Verbraucherfokus: Chancen für Ernährungsindustrie und Handel, Nürnberg.

Caswell, J.A., Joseph, S. (2008). Consumer Demand for Quality: Major Determinant for Agricultural and Food Trade in the Future? Journal of International Agricultural Trade and Development 4 (1), 99-116.

Caswell, J.A., Mojduszka, E.M. (1996). Using informational labeling to influence the market for quality and in food products. American Journal of Agricultural Economics 78 (5), 1248-1253.

Caswell, J.A., Padberg, D.I. (1992). Toward a more comprehensive theory of food labels. American Journal of Agricultural Economics 74 (2), 460-468.

Chen, Y.-C., Shang, R.-A., Kao, C.-Y. (2009). The effects of information overload on consumers' subjective state towards buying decision in the internet shopping environment. Electronic Commerce Research and Application 8 (1), 48-58.

Darby, M.R., Karni, E. (1973). Free competition and the optimal amount of fraud. Journal of Law and Economics 16 (1), 67-88.

de la Hunty, A., Ashwell, M., Arens, U., Gibson, S., Sadler, M. (2014). Authorised Health Claims May Not Help Consumers to Choose a Healthy Diet. Annals of Nutrition and Metabolism 64 (1), 1-5.

Elbakidze, L., Nayga, R.M.J., LI, H. (2013). Willingness to pay for multiple quantities of animal welfare dairy products: results from random nth-, second-price, and incremental second-price auctions. Canadian Journal of Agricultural Economics 61 (3), 417-438.

Ellis, K.A., K. Billington, B. McNeil, D., McKeegan, E.F. (2009). Public opinion on UK milk marketing and dairy cow welfare. Animal Welfare 18 (3), 267-282.

European Commission (2009). Europeans' attitudes towards the issue of sustainable consumption and production. Flasheurobarometer, 256.

Fernqvist, F., Ekelund, L. (2014). Credence and the effect of consumer liking on food. Food Quality and Preference 32 (Part C), 340-353.

Feunekes, G.I.J., Gortemaker, I.A., Willems, A.A., Lion, R., van den Kommer, M. (2008). Front-of-pack nutrition labelling: Testing effectiveness of different nutrition labelling formats front-of-pack in four European countries. Appetite 50 (1), 57-70.

Fischer, C., Lyon, T.P., Arbor, A. (2012). Competing Environmental Labels. Washington and Ann Arbor.

Fischer, C., Lyon, T.P., Arbor, A. (2013). A Theory of Multi-Tier Ecolabels. 134th EAAE Seminar on "Labels on sustainability: an issue for consumers, producers, policy makers, and NGOs" | European Association of Agricultural Economists (EAAE) | Paris, France | March 22, 2013.

Grunert, K.G. (2005). Food quality and safety: consumer perception and demand. European Review of Agricultural Economics 32 (3), 369-391. 
Grunert, K.G., Wills, J.M. (2007). A review of European research on consumer response to nutrition information on food labels. Journal of Public Health 15 (5), 385-399.

Harbaugh, R., Maxwell, J. W., Roussillon, B. (2011). Label confusion: the groucho effect of uncertain standards. Management Science 57 (9), 1512-1527.

Harper, G.C., Makatouni, A., (2002) Consumer perception of organic food production and farm animal welfare. British Food Journal 104 (3), 287-299.

Harper, L., Souta, P., Ince, J., Mckenzie, J. (2007). Food labelling consumer research: what consumers want. A Literature Review. Food Standards Agency.

Hellberg-Bahr, A., N. Steffen, N., Spiller, A. (2012). Marketingpotentiale für Weidemilch. Jahrbuch der Österreichischen Gesellschaft für Agrarökonomie 21 (1), 3-12.

Jacoby, J., Speller, D.E., Kohn, C.A. (1974). Brand choice behavior as a function of information load. Journal of Marketing Research 11 (1), 63-69.

Jahn, G., Schramm, M., Spiller, A. (2005). The reliability of certification : quality labels as a consumer policy tool. Journal of Consumer Policy 28(1), 53-73.

Kehlbacher, A., Bennett, R., Balcombe, K., (2012). Measuring the consumer benefits of improving farm animal welfare to inform welfare labelling Food Policy 37 (6), 627-633.

Kelly, B. Hughes, C., Chapman, K., Louie, J.C.-Y., Dixon, H., Crawford, J., King, L., Daube, M., Slevin, T. (2009). Consumer testing of the acceptability and effectiveness of front-ofpack food labelling systems for the Australian grocery market. Health Promotion International 24 (2), 120-129.

Kolodinsky, J. (2012). Persistence of health labeling information asymmetry in the United States: historical perspectives and twenty-first century realities. Journal of Macromarketing 32 (2), 193-207.

Königstorfer, J., Gröppel-Klein, A. (2012). Wahrnehmungs- und Kaufverhaltenswirkungen von Nährwertkennzeichen auf Lebensmitteln. Marketing ZFP - Journal for Research and Management 34 (3), 213-226.

Kroeber-Riel, W., Esch, F.-R. (2004). Strategie und Technik der Werbung: Verhaltenswissenschaftliche Ansätze, Kohlhammer GmbH, Stuttgart.

Lagerkvist, C.J., Hess, S., (2010). A meta-analysis of consumer willingness to pay for farm animal welfare. European Review of Agricultural Economics 38 (1), 55-78.

Malhotra, N.K. (1982). Information load and consumer decision making. Journal of Consumer Research 8 (4), 419-430.

McCluskey, J.J., Lureiro, M.L. (2003). Consumer preferences and willingness to pay for food labeling: A discussion of empirical studies. Journal of Food Distribution Research 34 (3), 95-102.

Nelson, P. (1970). Information and consumer behaviour. Journal of Political Economy 78 (2), 311-329.

Nelson, P. (1974). Advertising as information. Journal of Political Economy 82 (4), 729-754.

Oxford Dictionary (2015). Policy. URL: http://www.oxforddictionaries.com/definition /english/policy. Abrufdatum: 01.02.2015.

Palma, M.A., Collart, A.J., Chammoun C.J. (2014). Information asymmetry in consumer perceptions of quality-differentiated food products. Journal of Consumer Affairs. Article first published online: 27 Nov 2014. DOI: 10.1111/joca.12053 
Pirog, R., (2004). Consumer perceptions of pasture-raised beef and dairy products: An Internet Consumer Study. Leopold Center, Iowa State University.

Procher, V., Vance, C. (2013). Who does the shopping? German time-use evidence, 1996 2009. Ruhr Economic Papers, No. 393. URL: http://dx.doi.org/10.4419/86788448. Abrufdatum: 14.11.2014.

Schulze, B., Spiller, A., Lemke, D. (2008). Glücksschwein oder arme Sau? Die Einstellung der Verbaucher zur modernen Nutztierhaltung. In Spiller, A., Schulze, B. (Hrsg.). Zukunftsperspektiven der Fleischwirtschaft - Verbraucher, Märkte Geschäftsbeziehungen, pp. 465-489. Universitätsverlag Göttingen, Göttingen.

Sørensen, H.S., Clement, J., Gabrielsen, G. (2012). Food labels - an exploratory study into label information and what consumers see and understand. The International Review of Retail, Distribution and Consumer Research 22 (1), 101-114.

Tietzel, M., Weber, M. (1991). Von Betrügern , Blendern und Opportunisten. Eine ökonomische Analyse. Zeitschrift für Wirtschaftspolitik 40 (2), 109-138.

Van Kleef, E., van Trijp, H., Paeps, F., Fernández-Celemín, L. (2008). Consumer preferences for front-of-pack calories labelling. Public Health Nutrition 11 (2), 203-13.

Verbeke, W. (2005a). Food quality policies and consumer interests in the EU. In: M. Klopčič, M. Kuipers, A., Hocquette, J-F. (Hrsg.): Consumer attitudes to food quality products: Emphasis on Southern Europe, S. 13-23.

Verbeke, W. (2005b). Agriculture and the food industry in the information age. European Review of Agricultural Economics 32 (3), 347-368.

Wies, R. (1994). Policy Definition and Classification: Aspects, Criteria, and Examples. Proceeding of the IFIP/IEEE International Workshop on Distributed Systems: Operations \& Management, Toulouse, France, 10 - 12 October 1994.

Zühlsdorf, A., Nitzko, S., Spiller, A. (2013). Kennzeichnung und Aufmachung von Lebensmitteln aus Sicht der Verbraucher: Empirische Untersuchungsbefunde, Göttingen. 


\section{Teil I: Potentials for multi-level food labelling systems}

I.1. Multi-level labelling: too complex for consumers?

Ramona Weinrich, Annabell Franz, Achim Spiller

Eingereicht bei Economia Agro-Alimentare 


\section{Teil I: Potentials for multi-level food labelling systems}

I.2 Developing food labelling strategies: multi-level labelling

Autoren: Ramona Weinrich, Achim Spiller

Eingereicht beim Journal of Cleaner Production 


\section{Teil I: Potentials for multi-level food labelling systems}

I.3 Zahlungsbereitschaftsanalysen in mehrstufigen Labelsystemen. Das Beispiel Tierschutzlabel

Autoren: Ramona Weinrich, Annabell Franz, Achim Spiller

Veröffentlicht bei Berichte über Landwirtschaft, 2014: 92 (2), 1-19,

DOI: http://dx.doi.org/10.12767/buel.v92i2.45, Herausgeber: Bundesministerium für Ernährung und Landwirtschaft, Referat 531, Postfach 1402 70, D-53107 Bonn 


\section{$1 \quad$ Einleitung}

Die landwirtschaftliche Nutztierhaltung ist eine aktuelle und kontrovers diskutierte Fragestellung. Besonders aus Verbrauchersicht ist die Thematik negativ besetzt und wird oft unter dem Begriff „Massentierhaltung“ diskutiert (8). Tatsache ist, dass die Haltungsbedingungen und damit das Tierwohl sich innerhalb der einzelnen Betriebsformen und Betriebe stark unterscheiden. Neben der konventionellen Landwirtschaft hat sich die ökologische Bewirtschaftung etabliert, deren Fleisch- und Wurstprodukte eine Alternative zu Produkten aus der dominierenden konventionellen landwirtschaftlichen Tierhaltung sein können. Doch der Konsum biologisch produzierter Lebensmittel bleibt anteilsmäßig sehr gering. Der Marktanteil für Schweinefleisch aus ökologischer Landwirtschaft lag 2012 bei 0,8 \% (4). Eine weitere Möglichkeit für Verbraucher, sich durch ihren Konsum für mehr Tierwohl in der Landwirtschaft einzusetzen, ist die Etablierung eines neuen Marktsegments: Ein landwirtschaftliches Nutztierhaltungssystem, dessen Vorgaben über den gesetzlichen Tierschutzrichtlinien liegen, ohne ökologische Futtermittel einzusetzen. Die Nachfrage nach solchen preiswerteren „TierschutzProdukten“"wird national auf etwa $20 \%$ geschätzt (36).

Fleisch und Fleischprodukte, die einem tierfreundlicheren Haltungssystem entstammen, können derzeit am besten durch ein privatwirtschaftliches Label vermarktet werden (11). Labelling (englisch für Kennzeichnung) ist ein besonders sinnvolles Instrument, wenn vom Gesetzgeber verschiedene Standards erlaubt sind (17). Es vermittelt dem Konsumenten auf schnellstem Wege Informationen zum Produkt und zum Produktionsprozess. Die potenziellen Vorteile eines Tierschutzlabels liegen darin, dass neben einer hohen Glaubwürdigkeit, wie im BioSegment, auch eine hohe Marktdurchdringung erzielt werden kann (44).

In Deutschland sind in jüngster Vergangenheit solche Tierschutz-Produkte auf den Markt gekommen (bspw. die Label „Für mehr Tierschutz“, „Tierschutz kontrolliert“ oder „Aktion Tierwohl“). In anderen Ländern gibt es Tierschutzlabel schon länger, wie etwa in den Niederlanden das Label „Beter Leven“ oder im Vereinigten Königreich die Label „Freedom Food“ sowie „Specially Selected Pork“. In der Schweiz gibt es das privatwirtschaftliche Label „TerraSuisse“ von Migros, das seit 1989 vertrieben wird, sowie seit 2007 „Naturafarm“ des Lebensmitteleinzelhändlers Coop. Weiterhin gibt es in den USA seit 2008 das fünfstufige Programm „Global Animal Partnership“.

Im Folgenden werden nach einem Literaturüberblick über die Zahlungsbereitschaft der Verbraucher für Produkte aus besonders tiergerechter Haltung die Unterschiede in den Haltungsformen des Labels „Für mehr Tierschutz“ mit einer Einstiegsstufe und einer Premiumstufe 
erläutert. Hierbei wird insbesondere auf die dadurch entstehenden zusätzlichen Kosten eingegangen. Im Anschluss daran werden zwei empirische Analysen vorgestellt, in denen das Verbraucherverständnis des zweistufigen Labels abgefragt wurde. Die zentralen Fragestellungen dieses Beitrags lauten: Wird durch eine Auszeichnung der Produkte mit dem höherwertigen Label der Premiumstufe eine entsprechend höhere Zahlungsbereitschaft erlangt? Ist die Stufigkeit des Labels „Für mehr Tierschutz“ allein durch seine grafische Darstellung für die Verbraucher nachvollziehbar?

Der Artikel schließt mit einer Diskussion sowie Schlussfolgerungen aus den Untersuchungsergebnissen. Er leistet damit einen Beitrag zur Analyse der in Politik wie Wirtschaft zunehmend diskutierten Konzeption mehrstufiger Label, wie es für den Food-Sektor unter anderem von den wissenschaftlichen Beiräten für Verbraucher- und Agrarpolitik in ihrem Gemeinschaftsgutachten gefordert wurde (11).

\section{Mehrstufigkeit in Labelsystemen}

Im internationalen Vergleich liegt die Besonderheit der beiden o. g. deutschen Label „Tierschutz-Kontrolliert“ und „Für mehr Tierschutz“ darin, dass sie zweistufig sind. Das heißt, es gibt jeweils zwei Level mit unterschiedlich strengen Anforderungen: Eine Einstiegsstufe, die gegenüber der am Markt dominierenden Haltungsform nur relativ geringfügige Investitionen der Landwirte notwendig macht, und eine Premiumstufe mit höheren Anforderungen, die entsprechend größere Investitionen erfordern. Den Landwirten wird es einerseits erleichtert, ihre Haltungssysteme umzustellen, andererseits kann der Markt nach der Preisbereitschaft der Konsumenten segmentiert werden.

Insgesamt gewinnen mehrstufige Systeme im Bereich der Kennzeichnung von Vertrauenseigenschaften, d. h. Eigenschaften, die nicht durch Endverbraucher zu überprüfen sind, zunehmend an Bedeutung. Als Vorreiter und etablierte Systeme können hier die Hotel- und Restaurantklassifizierung durch Sterne oder die Energieklassenkennzeichnung bei Haushaltsgroßgeräten angeführt werden. Bereits seit 1996 wird in Deutschland durch die DEHOGA (Deutscher Hotel- und Gaststättenverband e. V.) das Sternesystem zur Hotelklassifizierung genutzt. Ein Stern steht für „Tourist“, fünf Sterne bedeuten „Luxus“. Es gibt 270 Kriterien, die an Hand eines Punktesystems bewertet werden. Mindestkriterien sind zu erfüllen und eine Mindestpunktzahl ist zu erreichen. Das Klassifizierungssystem gewährleistet für die Gäste Transparenz und Sicherheit (10) und signalisiert einen weltweit anerkannten Hotelstandard (9). Mit je mehr Sternen ein Hotel ausgezeichnet ist, desto mehr Qualität erwartet der Gast. Entsprechend korrelieren die Anzahl der Sterne und höhere Preise positiv (1). Ein weiteres Beispiel 
Tierschutzlabel

ist das dreistufige Sternesystem in der Gastronomie. Das französische Klassifizierungssystem Michelin Guide umfasst die Auszeichnung eines Restaurants mit einem, zwei oder drei Sternen. Ausgezeichnete Restaurants werden auch überregional von Gästen aufgesucht, wenn sie mit einem oder mehreren Sternen prämiert sind (29). Es besteht ein Zusammenhang zwischen der Auszeichnung mit einem oder mehreren Sternen und einer anschließenden Preiserhöhung in den entsprechenden Restaurants (39).

Die Kennzeichnung von Energieklassen ist das dritte Beispiel für ein funktionierendes mehrstufiges Labelsystem. Sieben verschiedene Energieeffizienzklassen signalisieren Verbrauchern die relative Höhe des Stromverbrauchs: An den farbigen Balken von dunkelgrün über gelb und orange zu rot und der Einteilung in A+++ für die sparsamste Energieklasse bis D für die Energieklasse, deren Haushaltsgeräte relativ gesehen am meisten Strom verbrauchen. Das Label muss verpflichtend auf Fernsehgeräten und Haushaltsgeräten wie Waschmaschinen oder Trocknern angebracht werden (12). HEINZLE und WÜSTENHAGEN (23) fanden in diesem Zusammenhang heraus, dass eine Skala von A bis G von Verbrauchern besser verstanden wird als die Einteilung von A+++ bis D. Findet die zweite Klassifizierung Anwendung, so ist hier der Preis ein wichtigeres Kaufkriterium als die Höhe des Energieverbrauchs. Bei der Einteilung von A bis $\mathrm{G}$ werden diese Kriterien im Entscheidungsprozess etwa gleich gewichtet.

Insgesamt deuten die Beispiele an, dass ein mehrstufiges Label ein sinnvolles Instrument der Markt- und Preisdifferenzierung darstellen kann. In einem mehrstufigen System ist es notwendig, dass die Zahlungsbereitschaft (ZB) der Konsumenten für die jeweiligen Segmente erhoben wird. Nur so kann sichergestellt werden, dass für die neuen Produkte eine hinreichende ZB besteht und die Preisabstände richtig gewählt sind. 


\section{$3 \quad$ Zahlungsbereitschaft für Tierschutz-Produkte}

\subsection{Zahlungsbereitschaft für Tierwohl}

Die differierende ZB für Produkte mit unterschiedlichen Qualitätsleveln wird durch wissenschaftliche Studien gestützt $(42 ; 45)$. Auch Produkte aus besonders tiergerechter Haltung bieten für den Konsumenten eine (subjektiv wahrgenommene) höhere Qualität als das konventionelle Standardangebot an Fleisch- und Wurstwaren. Hier können nach SPILLER und ScHULZE (40) als Kaufmotive neben der ökologischen Erzeugung auch kontrollierte Qualität, Regionalität, eine extensive Haltungsform und eine besonders artgerechte Tierhaltung angebracht werden. Auch SCHÄFER (34) fand heraus, dass die Qualität ein entscheidendes Kaufkriterium bei Bio-Fleisch darstellt. Für österreichische Verbraucher ist ebenfalls die Qualität das wichtigste Konsumkriterium für Bio-Lebensmittel (43). Dass Absatzmöglichkeiten für TierschutzProdukte vorhanden sind $(15 ; 21 ; 22 ; 28)$, hat auch die Privatwirtschaft in Deutschland erkannt. Seit Juni 2011 kann Fleisch mit dem Label „Aktion Tierwohl“ gekauft werden, seit Januar 2013 können Verbraucher in ausgewählten Regionen Fleisch und Wurst mit den Labeln „Für mehr Tierschutz“ und „Tierschutz-Kontrolliert“" erwerben.

Die Preise für die Produkte dieser Labelsysteme müssen unter denen für vergleichbare Produkte aus ökologischer Tierhaltung liegen, um Tierschutz-Produkte am Markt konkurrenzfähig zu machen. Verschiedene Arbeiten haben sich mit der ZB für Produkte aus tierfreundlichen Haltungssystemen beschäftigt. In einer Studie im Auftrag der Europäischen Kommission (18) geben $53 \%$ der Verbraucher an, dass sie im Supermarkt Schwierigkeiten haben, Informationen zu tierfreundlichen Produkten zu erhalten. $54 \%$ geben an, dass ein Label allein nicht hilft, eine Unterscheidung zu konventionellen Produkten festzustellen. Als beste Möglichkeit zur Produktidentifizierung werden jedoch Texte und Kennzeichnungen auf Verpackungen genannt. Eine andere Studie der Europäischen Kommission (16) untersucht die Bereitschaft, einen Aufschlag für Tierschutz-Eier zu bezahlen. Im Durchschnitt geben $57 \%$ der Europäer an, einen Aufschlag zahlen zu wollen. $25 \%$ akzeptieren einen Mehrpreis von $5 \%$, $21 \%$ würden bis zu $10 \%$ mehr ausgeben und $11 \%$ sehen ihr Limit bei einem Mehrpreis von $25 \%$. Insbesondere skandinavische und niederländische Konsumenten sind bereit, mehr Geld für Tierschutz-Produkte auszugeben, gefolgt von Deutschen, Luxemburgern und Österreichern. Eine geringe Bereitschaft findet sich in den neuen und südlichen EU-Ländern wie Griechenland oder Spanien. In einer Studie von KEHLBACHER et al. (26) wird nachgewiesen, dass in Großbritannien mit steigendem Tierschutz in der Landwirtschaft auch die ZB steigt. BENNETT und THOMPSEN (6) haben ebenfalls eine Befragung zu Zahlungsbereitschaften briti- 
scher Bürger durchgeführt. Dabei wurde eine Skala von 0 bis 100 Welfare Scores benutzt, ein Wert von 40 Welfare Scores stellt dabei den gesetzlichen Mindeststandard dar. Für Welfare Scores von 60 bis 80 ergibt sich eine marginale ZB von $£ 2,59$ je Welfare Score und Jahr. Für Scores von 70 bis 90 ergibt sich eine ZB von $£ 1,36$. Dieses Ergebnis wirkt zunächst überraschend, wurde von den Autoren jedoch so erwartet und spiegelt auch das Gesetz des abnehmenden Grenznutzens wider.

NORWOOD und LUSK (33) befassten sich mit der hypothetischen und der tatsächlichen ZB. Während 63,5\% der kalifornischen Verbraucher eine Abschaffung der Haltung von Hennen in Käfigen befürworten, weisen Scannerdaten aus San Francisco auf, dass nur $10 \%$ der verkauften Eier aus Bio-, Boden- oder Freilandhaltung stammen. AKAICHI et al. (5) haben sich mit den Tierschutzlabeln „Freedom Food“ und „Specially Selected Pork“ beschäftigt. Sie fanden heraus, dass für schottische Verbraucher der Preis von so gelabelten Produkten zu konventionellen Produkten konkurrenzfähig sein muss, der Aufpreis also nicht zu hoch angesetzt sein darf. Speziell zur ZB bei Schweinefleisch fanden MiLLER und UnNEVEHR (30) heraus, dass vor allem bei Frauen und älteren Konsumenten eine höhere ZB besteht.

Auch ist die ZB in Städten höher als in ländlichen Gebieten. Insgesamt haben die Befragten, die mehr Fleisch essen, weniger Bedenken bzgl. Herkunft und Sicherheit von Schweinefleisch. GRANNis und ThILMANY (20) haben eine Befragung zur ZB bei PremiumSchweinefleisch mit US-amerikanischen Verbrauchern durchgeführt. 29,7\% der Probanden gaben an, dass sie für einen Premium-Schinken einen Aufpreis von $10 \%$ zahlen würden. Einen Aufpreis von $20 \%$ akzeptierten 6,25 \% der Befragten. Für Hackfleisch ist die ZB höher. Einen Mehrpreis von $10 \%$ akzeptieren $40 \%$ der Befragten, 14,2\% halten einen Mehrpreis von $20 \%$ für angemessen. Nur ein sehr kleiner Anteil von unter $5 \%$ ist bereit, für eines der beiden Produkte einen Aufpreis von 30 bis $50 \%$ zu bezahlen.

NoCELla et al. (32) haben die absoluten Wochenausgaben für Lebensmittel in verschiedenen europäischen Ländern untersucht. Die Probanden wurden gefragt, wie viel mehr sie pro Woche für Tierschutz-Fleisch ausgeben würden. In Deutschland liegt der Mehrpreis bei 8,11€, im Vereinigten Königreich bei 9,11€. In südlichen EU-Ländern lag der akzeptierte Aufschlag auf die Ausgaben für den Wocheneinkauf höher, so wird in Italien ein Aufpreis von 11,81€ akzeptiert, in Spanien sogar von 13,85€. Eine Metanalyse zur ZB von LAGERKVIST und HESS (27) hingegen widerlegt die regional unterschiedlichen ZB. In der Studie belegen die Autoren jedoch, dass Informationen über die Haltungsbedingungen die ZB erhöhen (ebd.). 
Die bestehende Literatur verdeutlicht, dass es bei der Einführung von Labelsystemen, denen tierschutzfreundlichere Richtlinien zu Grunde liegen, viele Barrieren gibt, die nicht ohne weiteres überwunden werden können. Die (bekundete) ZB ist eher moderat. Um eine entsprechende ZB realisieren zu können, ist es unerlässlich, ein Tierschutzlabel umfassend zu kommunizieren. Vor diesem Hintergrund werden die Ergebnisse von zwei eigenen Studien dargestellt, die das Ziel hatten, das Verständnis des deutschen Tierschutzlabels „Für mehr Tierschutz" und die entsprechende ZB für dessen Einstiegs- und Premiumstufe zu analysieren.

\subsection{Kosten und Preise im Tierschutzsegment}

\subsubsection{Anforderungen in der landwirtschaftlichen Produktion}

Die Wahl des Labels für die hier vorgestellten Studien fiel auf das zweistufige Zeichen „Für mehr Tierschutz“, weil es derzeit die umfangreichsten und detailliertesten Richtlinien auf dem deutschen Markt aufweist. Zudem ist der Träger des Labels, der Deutsche Tierschutzbund, eine neutrale Organisation, die eine große Reichweite und einen hohen Bekanntheitsgrad hat. Darüber hinaus wurde das Label durch einen Multi-Stakeholder-Ansatz entwickelt und von Beginn an durch einen Labelbeirat begleitet. Dieser setzt sich aus Vertretern von NGOs (nongovernmental organisations), der Wissenschaft sowie der einzelnen Wertschöpfungsstufen Landwirtschaft, Schlachtung und Handel zusammen (13).

Im Folgenden werden beispielhaft die Anforderungen dargelegt, die ein Haltungssystem für Schweine erfüllen muss, damit die Produkte das Tierschutzlabel „Für mehr Tierschutz“ des Deutschen Tierschutzbundes tragen können. Auf Grund dieser Anforderungen erhöhen sich die Kosten für das Endprodukt. Die Anforderungen für beide Labelstufen gehen über die gesetzlichen Mindeststandards des Tierschutzes hinaus. Die erste Stufe hat dabei geringere Vorgaben als die zweite Stufe. So soll es Landwirten erleichtert werden, ihre Haltungssysteme schrittweise tierfreundlicher umzugestalten.

Abbildung 1 zeigt die grafische Darstellung der beiden Labelstufen. Das Label der sog. Einstiegsstufe ist dabei durch einen gelben Stern und einen zweiten, abgeblassten Stern gekennzeichnet. Letzterer soll auf das Premium-Produkt mit strengeren Richtlinien, das mit zwei Sternen gekennzeichnet ist, hinweisen. 


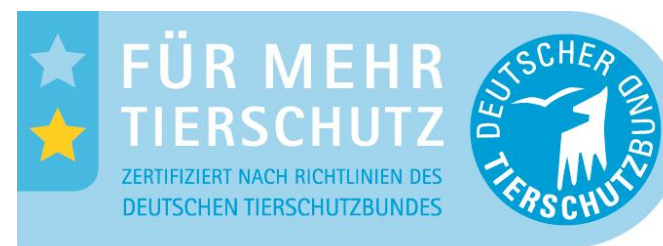

tierschutzlabel.info Einstiegsstufe

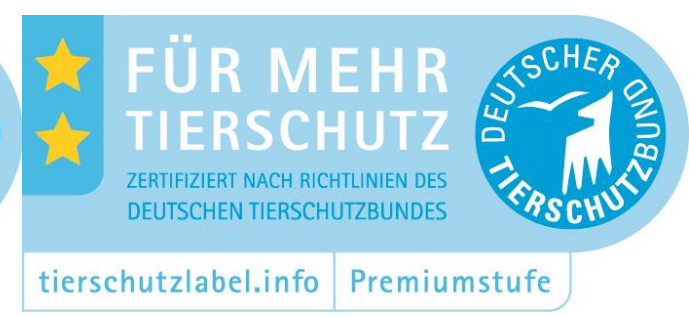

Abb. 1: Das zweistufe Label „Für mehr Tierschutz“ des Deutschen Tierschutzbunds (links: Einstiegsstufe, rechts Premiumstufe)

Die Richtlinien, die dem Label zu Grunde liegen, umfassen alle Stufen der Produktion, beginnend bei dem landwirtschaftlichen Betrieb über den Transport bis hin zur Schlachtung. Die Mehrkosten, die sich für den Landwirt aus den Änderungen der Haltungsanforderungen ergeben, setzen sich vor allem aus den Umbau- und Ausbaumaßnahmen, der Zertifizierung, dem erhöhten bürokratischen Aufwand, wie Kontrollen und Dokumentation, und der zusätzlichen Stallarbeit zusammen. Beim Transport fallen Kosten auf Grund kürzerer Transportzeiten (und damit verbunden einer geringeren Auslastung der Fahrzeuge) sowie einem erhöhten Platzangebot für die Tiere im Transportfahrzeug an. Bei der Schlachtung steigen die Kosten insbesondere auf Grund der Dokumentation und den Anforderungen an den Umgang mit den Tieren.

Eine prägnante Kennzahl ist in den landwirtschaftlichen Betrieben der Platz, der pro Tier zur Verfügung steht. Gesetzlich vorgeschrieben sind 0,75 qm pro Schwein. In der Endmast stehen einem Tier in der Einstiegsstufe hingegen 1,1 qm zur Verfügung, in der Premiumstufe sind es 1,5 qm. Insgesamt darf ein Betrieb der Einstiegsstufe max. 3.000 Mastplätze haben, ein Betrieb, der Schweine für die Premiumstufe mästet, nur 950 Mastschweine halten. Neben einem erhöhten Platzangebot für die Tiere muss auch ein planbefestigter Liegebereich, der in der Premiumstufe mit Stroh eingestreut sein muss, zur Verfügung stehen. Den Tieren in der Premiumstufe muss zusätzlich ein Auslauf im Freien von 0,5 qm zugänglich sein. Alternativ ist der Kontakt zum Außenklima durch Netze statt Wände zugelassen. Der zusätzliche Quadratmeteranteil des Auslaufs wird dann auf die Stallfläche angerechnet. Die Fütterung mit gentechnisch verändertem Futter ist für Tiere des zwei Sterne-Labels verboten. Für den Einstiegsbereich gibt es eine Übergangsfrist von drei Jahren. Ebenso verboten ist die betäubungslose Kastration von Ferkeln. Auch das Kürzen der Schwänze ist unzulässig. Hier gibt es ebenfalls eine Übergangsfrist: Bis Ende 2015 darf ein Drittel des Schwanzes gekürzt werden. Die Transportdauer vom Betrieb zum Schlachthof ist auf vier Stunden und max. $200 \mathrm{~km}$ begrenzt. Zwischen der Ankunft am Schlachthof und dem Abladen des ersten Tieres dürfen max. 60 Minuten liegen. Auf dem Schlachthof muss ein Wartestall vorhanden und eine sichere Betäu- 
bung vor dem Schlachten gewährleistet sein. Tierbezogene Kriterien wie Verletzungen, Verluste oder Notschlachtungen müssen auf allen drei Stufen dokumentiert werden. Durch die höheren Auflagen entstehen folglich höhere Kosten (14).

\subsubsection{Mehrkosten in der landwirtschaftlichen Produktion}

Bisher gibt es nur wenige Studien zu den zusätzlich entstehenden Kosten auf Grund der höheren Anforderungen und der Verteilung der Kosten entlang der Wertschöpfungskette. Erste Schätzungen von ACHILLES und FRITZSCHE (2) ergeben, dass die Mehrkosten in der Produktion für den Einstiegsstandard des Tierschutzlabels um 0,20€ je kg SG steigen. Hierbei ist zu beachten, dass nicht alle Kosten durch die veränderten Haltungsbedingungen einbezogen sind. Nach einer Kalkulation von HEMPLER (24) liegt die Differenz zwischen konventioneller Erzeugung und dem 1-Sterne-Standard bei $0,15 €$.

Eine Studie der Universität Wageningen (25) beschäftigt sich mit dem Einfluss von zusätzlichem Platz für die Schweine ab $85 \mathrm{~kg}$ auf die Kosten. Wenn der Landwirt einen Anbau vornehmen kann, um den zusätzlichen Platzbedarf zu decken, erhöhen sich die Kosten um 2,4 Cent je kg SG (Platzangebot erhöht sich von 0,7 auf $1 \mathrm{qm}$ ) oder 1,5 Cent je kg SG (Erhöhung von 0,8 auf $1 \mathrm{qm})$. Muss allein das vorhandene Platzangebot ausreichen, würden sich analog die Kosten je kg SG um 5,6 Cent (Platzangebot erhöht sich von 0,7 auf 1 qm) bzw. 8,4 Cent erhöhen (Erhöhung von 0,8 auf 1 qm).

Auf Grund der wenigen vorhandenen Studien werden in diesem Artikel ebenfalls Zahlen und Literatur aus dem Bio-Bereich und des Neuland-Vereins heranzogen, um Kosten- und Preisunterschiede für das 2-Sterne-Segment abzuschätzen. Das Neulandprogramm besteht seit 1988 und hat strengere Richtlinien, als die gesetzlichen Vorgaben es vorschreiben. Im Unterschied zur Bio-Haltung müssen die Tiere nicht mit ökologischem Futter gefüttert werden. Nach Hempler (24) liegen die Mehrkosten für eine Produktion nach Neuland-Richtlinien bei $0,77 €$ je kg SG und für den Bio-Standard bei $1,70 €$ je kg SG. In der Studie von ACHILLES und FRITZSCHE (2) erhöhen sich die Kosten für den 2-Sterne-Standard um 0,35 € je kg SG und für die Einhaltung der Bio-Richtlinien um 1,65€ je kg SG.

BEUKERT und SimON (7) haben eine Modellrechnung aufgestellt, deren Kosten sich an der EG-Öko-VO orientieren. Demnach entstehen zusätzliche Kosten von etwa $1 € /$ kg Schlachtgewicht (SG) bei Schweinefleisch. Die Vorschriften der EU-Öko-Verordnung führen vor allem zu höheren Kosten beim Futter und bei der Arbeitskraft. Aus den Bestimmungen der EGÖko-Basisverordnung (EG) Nr. 834/2007 entstehen in Schlachtung, Verarbeitung und Ver- 
Tierschutzlabel

marktung nur geringfügig höhere Kosten. Laut BEUKERT und SIMON (7) ist eine Quantifizierung der Kosten nicht möglich, da keine belastbaren Daten dazu vorliegen.

Nach den Angaben der AMI für die Marktdaten Öko-Landbau (3) lag der Erzeugerpreis für Öko-Mastschweine pauschal im Jahr 2012 durchschnittlich bei 3,15€ je kg SG, im gleichen Jahr lag der Durchschnittspreis für konventionelle Schweine bei 1,74€ je kg SG (4).

\subsubsection{Mehrkosten und Preise auf den nachgelagerten Stufen der Werstschöpfungskette}

Da keine Kostendaten für die nachgelagerten Stufen in der Wertschöpfungskette vorhanden sind, werden daher hilfsweise Verbraucherpreise als Indikator verwendet.

Der recht hohe Preis für den Endverbraucher, eine geringe Distributionsdichte und Zweifel an der Echtheit der Produkte hemmen die Ausweitung des Marktanteils für ökologisches Schweinefleisch (37). Das geringe Marktvolumen führt wiederum zu zusätzlichen Kosten im Handel: die Umschlagshäufigkeit ist gering, was zu steigenden Opportunitätskosten führt. Außerdem entstehen Verluste durch häufiges Überschreiten des Mindesthaltbarkeitsdatums, sodass Produkte vernichtet werden müssen (38). Ein weiterer Grund ist, dass oft nur die Edelteile als Biofleisch verkauft werden und die übrigen Teile als konventionelles Fleisch vermarktet werden (Kuppelproduktionsproblematik). Aus diesem Grund liegen die Preisunterschiede beim Endverbraucher um ein Vielfaches höher als die eigentlichen Mehrkosten, die in der landwirtschaftlichen Produktion bestehen.

Eigene Recherchen haben ergeben, dass das Tierschutz-Fleisch der Einstiegsstufe im Lebensmitteleinzelhandel beim Vollsortimenter etwa $1 €$ je kg Schweinefleisch mehr kostet als konventionelle Vergleichsprodukte. Zu beachten ist dabei, dass bei einer Preiserhöhung von Biofleisch die Nachfrage nach Biofleisch sehr sensibel regiert: Steigt der Preis um $1 \%$, so sinkt die Nachfrage ceteris paribus um $2 \%$ (35). Daher stellt sich die Frage, wie Konsumenten in der Nachfrage nach Tierschutzfleisch reagieren. Eine Übersicht über ausgewählte Produkte gibt Tabelle 1. 
Tabelle 1: Preis für Schweinefleisch nach eigener Recherche bei ausgewählten Einzelhändlern in $€ / \mathrm{kg}$ (Stand: April/ Mai 2013)

\begin{tabular}{|c|c|c|c|}
\hline Markt & Teilstiick & Konventionell & Tierwohl 1-Stern \\
\hline Kaiser's Tengelmann & Filet & 6,90 (Angebot) & 16,90 \\
\hline Kaiser's Tengelmann & Kotelett & 8,99 & 9,99 \\
\hline Edeka & Nacken & 7,98 & 8,99 \\
\hline Edeka & Schnitzel & 8,90 & 9,99 \\
\hline Sky & Nacken & 7,29 & 7,99 \\
\hline Sky & Kotelett & 8,49 & 4,44 (Angebot) \\
\hline
\end{tabular}

\section{Quelle: eigene Recherche}

Tabelle 1 zeigt deutlich abweichende Preisunterschiede, sobald ein Produkt mit einem Aktionspreis beworben wird. Da es derzeit fast keine Produkte mit dem Premiumlabel zu erwerben gibt, können hier keine Aussagen über Preise und Kosten getroffen werden.

Die Preise für den Endverbraucher werden aber nach Schätzungen deutlich über den entstehenden Mehrkosten in der Produktion liegen. Die Gründe dafür sind ähnlich gelagert wie bei Bio-Fleisch: eine geringe Distributionsdichte, die niedrige Umschlagshäufigkeit, die damit verbundenen Opportunitätskosten, die Kuppelproduktionsproblematik und der hohe Anteil an verderbender Ware treiben Kosten und Preise in die Höhe. Wenn mehr Tierschutzfleisch vermarktet werden kann,können die Preise für den Endverbraucher sinken. Der Mehrpreis, den er bezahlt, kommt dann zu einem größeren Anteil dem Tier zu Gute, womit die ZB durch entsprechende Kommunikationsmaßnahmen besser erreicht werden kann.

Die folgenden zwei Fallstudien analysieren, wie hoch die Zahlungsbereitschaften für Produkte mit dem Tierschutzlabel ausfallen, und wie wichtig dabei die Kommunikation bei der Etablierung des Labels ist.

\section{Case Study Tierschutzlabel}

Im Folgenden werden zwei empirische Studien vorgestellt, in denen jeweils Zahlungsbereitschaften zum Tierschutzlabel abgefragt wurden. Es wird aufgezeigt, wie die Kommunikation zu einem Label die Zahlungsbereitschaft bei den Konsumenten beeinflusst. Abbildung 2 zeigt das Label in einem früheren Design, welches zum Zeitpunkt der ersten Befragung in 2011 verwendet wurde. 

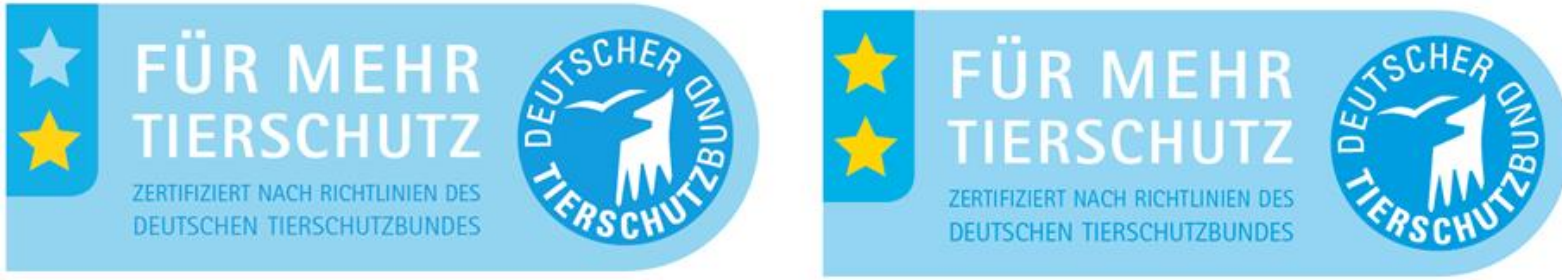

Abb. 2: Das zweistufige Label „Für mehr Tierschutz“ des Deutschen Tierschutzbunds in der für die Studie verwendeten Aufmachung (links: Einstiegsstufe, rechts: Premiumstufe)

Der Unterschied zu dem neueren Design (vgl. Abb. 1) ist der fehlende Zusatz am unteren Rand des Labels. Hinzugefügt wurde der Verweis auf die Website des Tierschutzlabels „tierschutzlabel.info“" sowie rechts davon die zusätzliche Information Einstiegs- bzw. Premiumstufe.

\subsection{Studie 1: Zahlungsbereitschaftsanalyse mit Aufklärung über die Stufigkeit des Labels „Für mehr Tierschutz“6}

\subsubsection{Material und Methoden}

Die Befragung fand zwischen dem 31. August und dem 5. September 2011 statt. Die Stichprobe umfasst 306 Haushaltsentscheider aus Deutschland. Die Probanden wurden mit Hilfe eines Online-Access-Panels rekrutiert. Im Rahmen dieser Studie wurden den Probanden nach einleitenden ersten Fragen Informationen über die Stufigkeit des Labels „Für mehr Tierschutz" in folgender Form gegeben: An dieser Stelle möchten wir Sie über das eben gezeigte Siegel des Deutschen Tierschutzbundes aufklären. Es handelt sich hierbei um ein freiwilliges Tierschutz-Siegel, das voraussichtlich Ende des Jahres auf den Markt kommen wird, um das aktuelle Angebot von Lebensmitteln tierischen Ursprungs zu ergänzen. Das Siegel wird Produkte kennzeichnen, die unter besonders tiergerechten Bedingungen produziert wurden. Das heißt, die Tiere wurden unter Bedingungen gehalten, die deutlich über die gesetzlichen Mindestvorschriften hinausgehen. Diese Bedingungen werden vom Deutschen Tierschutzbund vorgegeben und ihre Einhaltung durch unabhängige, externe Prüfinstitute kontrolliert. Das Siegel wird Produkten in zwei verschiedenen Kategorien verliehen werden:

1) Die Produkte der ersten Kategorie erfüllen Kriterien, die über die gesetzlichen Vorgaben hinausgehen (größeres Platzangebot, kurze Transportwege u.a.) und werden mit EINEM Stern ausgezeichnet (linkes Bild).

2) Produkte der zweiten Kategorie erfüllen noch höhere Bedingungen (zusätzlicher Auslauf ins Freie für die Tiere u.a.) und werden daher mit ZWEI Sternen ausgezeichnet (rechtes Bild). 
Anschließend wurden allen Teilnehmern Bilder von jeweils zwei identischen Fleisch- [Nackensteak (250 g)] oder Wurstprodukten [Bacon (150 g)] mit und ohne Label gezeigt. Links war jeweils das konventionelle Referenzprodukt ohne Label und rechts das Produkt mit dem Label der Einstiegs- oder der Premiumstufe. Auf dem Produkt ohne Label war jeweils der Preis angegeben, dieser diente als Referenzpreis. Die Teilnehmer wurden ungestützt nach ihrer Zahlungsbereitschaft für das Produkt mit dem Label gefragt. Alle bekamen die Produkte jeweils mit einem Stern und mit zwei Sternen nacheinander vorgelegt. Zusätzlich hatten sie die Möglichkeit anzugeben, dass sie die gezeigten Produkte generell nicht kaufen (no choice option).

\subsubsection{Stichprobenbeschreibung}

Die 306 Teilnehmer setzen sich entsprechend der Quotenvorgabe (70\% Frauen und $30 \%$ Männer) aus 69,9 \% Frauen und 30,1 \% Männern zusammen, sodass die Geschlechterverteilung in der Stichprobe der Verteilung der Haushaltsentscheider in Deutschland entspricht. Das Durchschnittsalter liegt bei 41 Jahren. 55,5 \% haben einen Hochschulabschluss, was über dem nationalen Durchschnitt von $26 \%$ liegt (41). Tabelle 2 gibt einen detaillierteren Überblick über die soziodemografischen Eigenschaften der Stichprobe wieder.

Tabelle 2: Charakterisierung der Stichprobe

\begin{tabular}{|c|c|}
\hline Variable & Anteil \\
\hline Stichprobengröße & 306 \\
\hline Geschlecht & \\
\hline Männlich & $30,1 \%$ \\
\hline Weiblich & $69,9 \%$ \\
\hline Alter & $28,1 \%$ \\
\hline 18 bis 30 Jahre & $45,1 \%$ \\
\hline 31 bis 50 Jahre & $26,8 \%$ \\
\hline älter als 50 Jahre & \\
\hline Haushaltseinkommen & $54,4 \%$ \\
\hline Nettoeinkommen <2500 $€$ & $29,2 \%$ \\
\hline Nettoeinkommen > 2500 $€$ & $16,4 \%$ \\
\hline Keine Angabe & \\
\hline Bildung & $55,5 \%$ \\
\hline Hochschulabschluss & \\
\hline
\end{tabular}

Quelle: eigene Darstellung 
Tierschutzlabel

Die Stichprobe ist repräsentativ für die deutsche Bevölkerung bzgl. des Geschlechts der Haushaltsentscheider, die über den Kauf bzw. Nicht-Kauf von Tierschutz-Produkten entscheiden.

\subsubsection{Ergebnisse}

Vor der Präsentation der Informationen wurden die Teilnehmer ungestützt nach der Bedeutung der Sterne in dem Label gefragt. 16,1 \% verbinden die Sterne mit einem Ranking, einer Kategorisierung oder einer Bewertung. 12,6 \% der Teilnehmer können den Sternen keine Bedeutung zuordnen. $11 \%$ bringen die Sterne mit der EU in Verbindung oder messen ihnen eine internationale Bedeutung bei. $7 \%$ denken, dass die Sterne Qualität symbolisieren und 6,8 \% denken an eine Auszeichnung. Die übrigen Aussagen sind von weniger als $5 \%$ der Teilnehmer getroffen worden oder konnten nicht kategorisiert werden.

Vor der Aufklärung über die Bedeutung der Label geben $80 \%$ der Teilnehmer an, dass sie Produkte mit dem Label kaufen würden, nach der Aufklärung erhöht sich dieser Anteil auf $87 \%$. Weiterhin wurden eine Frage zur Vertrauenswürdigkeit und eine Frage zur Glaubwürdigkeit gestellt. $65 \%$ der Teilnehmer halten das Label des Deutschen Tierschutzbundes ohne Erklärung nicht für vertrauensvoll, nach der Aufklärung reduziert sich dieser Anteil auf $37 \%$. Auch die Ergebnisse der zweiten Frage lassen auf ein Vertrauensdefizit schließen: Vor der Aufklärung halten $23 \%$ das Zeichen für glaubwürdig, nach der Aufklärung über das Label erhöht sich die Glaubwürdigkeit auf $40 \%$. Während vor der Aufklärung 46,8 \% nicht sicher sind, für wie glaubwürdig sie das Label halten sollen, sind nach der Aufklärung nur noch $38,3 \%$ unsicher.

Im Anschluss daran wurden die Befragten über die Bedeutung des Tierschutzlabels aufgeklärt und zu ihrer ZB für die jeweiligen Produkte befragt.

Die folgenden Tabellen zeigen in der linken Spalte jeweils die Stufe des Labels. Die mittlere Spalte beinhaltet die durchschnittliche ZB, die rechte den Preisaufschlag gegenüber dem Referenzprodukt, der zusätzlich in Prozent berechnet wurde Tabelle 3 zeigt die ZB für Bacon. Diese ist 25,7 \% höher, wenn das Produkt aus einer Tierhaltung stammt, die den Vorgaben der Einstiegsstufe entspricht. Der Preisaufschlag beträgt 47,5\%, wenn das Produkt mit dem Label der Premiumstufe gekennzeichnet ist. 
Tabelle 3: ZB für Bacon (Referenzpreis: 1,79€/150 g)

\begin{tabular}{|c|c|c|c|}
\hline Standard & Durchschnittliche ZB & Preisaufschlag in \% & Standardabweichung \\
\hline Einstiegsstufe & $2,25 €$ & 25,7 & 0,55 \\
\hline Premiumstufe & $2,64 €$ & 47,5 & 0,83 \\
\hline
\end{tabular}

Quelle: eigene Darstellung, $\mathrm{n}=206-263$

Die folgende Tabelle beinhaltet die ZB für Nackensteak. Der akzeptierte Preisaufschlag ist höher im Vergleich zu Bacon: 39,4 \% für das Label der Einstiegsstufe und 66,7 \% für das Label der Premiumstufe.

Tabelle 4: ZB für Nackensteak (Referenzpreis: 2,49 € / $250 \mathrm{~g}$ )

\begin{tabular}{|c|c|c|c|}
\hline Standard & Durchschnittliche ZB & Preisaufschlag in \% & Standardabweichung \\
\hline Einstiegsstufe & $3,47 €$ & 39,4 & 1,14 \\
\hline Premiumstufe & $4,15 €$ & 66,7 & 1,72 \\
\hline
\end{tabular}

Quelle: eigene Darstellung, $n=260-263$

Die Preisunterschiede bei beiden Produkten sind jeweils signifikant auf dem 0,1\% Niveau, sodass eine systematische Preisdifferenz für die Premiumstufe erkennbar ist.

\subsection{Studie 2: Zahlungsbereitschaftsanalyse ohne Aufklärung über die Stufigkeit des Labels}

\subsubsection{Material und Methoden}

In einer weiteren Online-Studie zur ZB für Produkte mit dem Label „Für mehr Tierschutz“ wurde die Stichprobe in zwei Subsamples unterteilt. Der eine Teil der Stichprobe wurde zu seiner ZB zu Frischfleisch befragt, der andere Teil bekam die gleichen Fragen in Bezug auf Wurstprodukte gestellt. Das Subsample „Wurst“ hatte eine Größe von 318 Teilnehmern, 324 Probanden wurden dem Sample „Frischfleisch“ zugeordnet. Die Befragung fand im August 2012 statt. Um die Repräsentativität der Stichprobe zu gewährleisten, wurden Quoten im Hinblick auf das Geschlecht des Haushaltsentscheiders (70 \% Frauen, 30 \% Männer), das Nettohaushaltseinkommen und das Alter festgelegt (41).

Im Vergleich zu Fallstudie 1 wurden die Teilnehmer dieser Studie nicht über die Stufigkeit des Labels informiert. Sie wurden in beiden Subsamples zufällig in Gruppen eingeteilt, die entweder Produkte mit dem Label der Einstiegsstufe oder der Premiumstufe sahen. Die Darstellung der Produkte erfolgte entsprechend Fallstudie 1: Auf der linken Seite war das konventionelle Referenzprodukt, rechts war das jeweilige Produkt mit dem Label der Einstiegs- 
Tierschutzlabel

oder der Premiumstufe abgebildet. Die Teilnehmer sahen jeweils ein unverarbeitetes und ein verarbeitetes Produkt nacheinander. Im Subsample „Fleisch“ waren dies Kotelett (250 g) und Geschnetzeltes nach Gyros Art (400 g). Im Subsample „Wurst“ wurden Fotos der Produkte Nussschinken (150 g) und Leberwurst (125 g) vorgelegt. Vor der Befragung zur ZB erhielten die Probanden in Form eines Cheap-Talk-Skripts (19) den Hinweis, dass die hypothetische ZB oft überschätzt wird. Daher sollten sie sich eine realistische Einkaufssituation vorstellen und angeben, was sie wirklich zu zahlen bereit sind. Für jedes Produkt wurde die ZB je nach Gruppe für das Einstiegslabel oder das Premiumlabel ungestützt abgefragt. Zusätzlich hatten die Teilnehmer auch hier die Möglichkeit anzugeben, dass sie die gezeigten Produkte generell nicht kaufen.

\subsubsection{Stichprobenbeschreibung}

Die Stichprobe ist für die zuvor gesetzten Quoten Alter und Haushaltseinkommen repräsentativ. Ebenso ist die Stichprobe repräsentativ für das Geschlecht des Haushaltsentscheiders (Männer: Frischfleisch Befragung: 28,9\% [n=291], Wurst Befragung: 29,6\% [n=289]; Frauen: Frischfleisch Befragung: 71,1\%, Wurst Befragung: 70,4 \%). Das durchschnittliche Alter lag bei beiden Subsamples bei 46 Jahren. 27,6 \% der Teilnehmer in der Stichprobe „Fleisch“ und 25,1\% der Teilnehmer in der Stichprobe „Wurst“ haben einen Hochschulabschluss erworben. Tabelle 5 liefert detaillierte Informationen über die soziodemografischen Merkmale beider Subsamples. 
Tabelle 5: Charakterisierung der Stichprobe

\begin{tabular}{|c|c|c|}
\hline Variable & $\begin{array}{c}\text { Anteil (Fleisch-Sample; } \\
\text { in Prozent) }\end{array}$ & $\begin{array}{c}\text { Anteil (Wurst-Sample; in } \\
\text { Prozent) }\end{array}$ \\
\hline Stichprobengröße & 318 & 324 \\
\hline Geschlecht & $28,9 \%$ & $29,6 \%$ \\
\hline Männlich & $71,1 \%$ & $70,4 \%$ \\
\hline Weiblich & & $5,2 \%$ \\
\hline Alter & $2,4 \%$ & $32,9 \%$ \\
\hline 18 bis 20 Jahre & $31,3 \%$ & $35,3 \%$ \\
\hline 21 bis 39 Jahre & $42,6 \%$ & $26,6 \%$ \\
\hline 41 bis 59 Jahre & $23,7 \%$ & $14,8 \%$ \\
\hline 60 Jahre oder älter & & $22,5 \%$ \\
\hline Netto-Haushaltseinkommen & $11,9 \%$ & $30,9 \%$ \\
\hline Nettoeinkommen $<900 €$ & $24,8 \%$ & $23,8 \%$ \\
\hline 901 bis 1500 $€$ & $32,8 \%$ & $8,0 \%$ \\
\hline 1501 bis 2600 $€$ & $22,5 \%$ & $79 \%$ \\
\hline 2601 bis 4500 $€$ & $8,0 \%$ & $67 \%$ \\
\hline Nettoeinkommen $>4500 €$ & $75 \%$ & $25,1 \%$ \\
\hline Kinder unter 18 Jahren im HH & $64 \%$ & \\
\hline In einer Beziehung lebend / & & \\
\hline verheiratet & & \\
\hline Bildung & & \\
\hline Hochschulabschluss & & \\
\hline & & \\
\hline & & \\
\hline
\end{tabular}

Quelle: eigene Darstellung

\subsubsection{Ergebnisse}

78,3\% der Teilnehmer des Fleischfragebogens geben an, dass sie Produkte mit einem Tierschutzlabel kaufen würden; im Wurstsample sind es 77,7\%. Auch in dieser Befragung ist das Vertrauen in das Label eher verhalten. Auf einer 7-stufigen Likert Skala von -3 (trifft überhaupt nicht zu) bis +3 (trifft voll und ganz zu) liegt der Mittelwert bei 0,35 bei der Fleischbefragung, bei der Befragung zu Wurst liegt der Mittelwert bei 0,46. Danach gefragt, ob die Probanden ihr Kaufverhalten für mehr Tierschutz ändern würden, lässt sich bei Fleisch auf einer entsprechenden Likert Skala ein Mittelwert von 0,75 berechnen, bei Wurst ein Mittelwert von 0,52. 34,2\% der Teilnehmer geben an, dass sie einen Aufpreis für ein tierfreundlicher hergestelltes Kotelett zahlen würden, für Geschnetzeltes nach Gyros Art liegt der Anteil 
bei 14,6\%. Für Wurst ist die Bereitschaft, für mehr Tierschutz einen Aufpreis zu zahlen, geringer: $25,1 \%$ der Verbraucher würden mehr für einen tierfreundlicher hergestellten Nussschinken ausgeben, bei der Leberwurst liegt die Bereitschaft bei 14,1\%.

Tabelle 6 zeigt die durchschnittliche ZB für Kotelett. Die ZB für Produkte der Einstiegsstufe liegt 32,6\% über dem Referenzpreis. Für ein Produkt der Premiumstufe sind die Verbraucher bereit, einen Preisaufschlag von 32,2 \% zu zahlen. Die Ergebnisse verdeutlichen, dass die Zahlungsbereitschaft nahezu identisch ist. Dieses Ergebnis ist konträr zu den Resultaten der vorher vorgestellten Fallstudie, in der die Teilnehmer vor der Befragung über die Stufigkeit des Labels aufgeklärt wurden. Die Preisdifferenz zwischen dem 1-Sterne-Produkt und dem 2Sterne-Produkt ist statistisch nicht signifikant.

Tabelle 6: ZB für Kotelett (Referenzpreis: 2,49€/250 g)

\begin{tabular}{|c|c|c|c|}
\hline Standard & Durchschnittliche ZB & Preisaufschlag in \% & Standardabweichung \\
\hline Einstiegsstufe & $3,30 €$ & 32,6 & 0,84 \\
\hline Premiumstufe & $3,29 €$ & 32,2 & 1,35 \\
\hline
\end{tabular}

Quelle: eigene Darstellung, $n=70-72$

Ein ähnliches Ergebnis kann für das verarbeitete Fleischprodukt Geschnetzeltes nach Gyros Art beobachtet werden (Tabelle 7). Die ZB für das Premiumprodukt mit zwei Sternen ist sogar etwas niedriger als die für das Einstiegsprodukt mit einem Stern. Die ZB für die Einstiegsstufe ist 16,8 \% höher als der Referenzpreis. Der hypothetische Aufpreis für das Premiumprodukt liegt hingegen nur 12,7 \% höher als der angegebene Referenzpreis. Der Preisunterschiede für beide Labelstufen ist auch für das Geschnetzelte nach Gyros Art nicht signifikant.

Tabelle 7: ZB für Geschnetzeltes nach Gyros Art (Referenzpreis: 4,76€ / 400 g)

\begin{tabular}{|c|c|c|c|}
\hline Standard & Durchschnittliche ZB & Preisaufschlag in \% & Standardabweichung \\
\hline Einstiegsstufe & $5,56 €$ & 16,8 & 0,86 \\
\hline Premiumstufe & $5,37 €$ & 12,7 & 0,66 \\
\hline
\end{tabular}

Quelle: eigene Darstellung, $n=42-54$

Im Subsample „Wurst“ wurden die Zahlungsbereitschaften für die Produkte Nussschinken und Leberwurst getestet. Die Ergebnisse werden in den folgenden Tabellen 8 und 9 dargestellt. Im Fall des Nussschinkens ist die ZB für das Premiumprodukt höher als für das Einstiegsprodukt. Der Unterschied beträgt 4,9 Prozentpunkte zwischen Premium- und Einstiegs- 
produkt. Für den Nussschinken kann kein statistisch signifikanter Unterschied zwischen der ZB für das 1-Sterne-Produkt und der ZB für das 2-Sterne-Produkt ermittelt werden.

Tabelle 8: ZB für Nussschinken (Referenzpreis: 1,79€ / $150 \mathrm{~g}$ )

\begin{tabular}{|c|c|c|c|}
\hline Standard & Durchschnittliche ZB & Preisaufschlag in \% & Standardabweichung \\
\hline Einstiegsstufe & $2,21 €$ & 23,4 & 0,40 \\
\hline Premiumstufe & $2,30 €$ & 28,3 & 0,68 \\
\hline
\end{tabular}

Quelle: eigene Darstellung, $\mathrm{n}=76-81$

Die Ergebnisse für das verarbeitete Produkt Leberwurst (Tabelle 9) sind vergleichbar mit denen für Kotelett: Die ZB für das Premiumprodukt (2,11 €) ist fast identisch mit dem Wert für das Einstiegsprodukt $(2,12 €)$. Die ZB für die Produkte ist auch für die Leberwurst statistisch nicht signifikant unterschiedlich.

Tabelle 9: ZB für Leberwurst (Referenzpreis: $1,85 € / 125 \mathrm{~g}$ )

\begin{tabular}{|c|c|c|c|}
\hline Standard & Durchschnittliche ZB & Preisaufschlag in \% & Standardabweichung \\
\hline Einstiegsstufe & $2,12 €$ & 14,4 & 0,26 \\
\hline Premiumstufe & $2,11 €$ & 13,9 & 0,35 \\
\hline
\end{tabular}

Quelle: eigene Darstellung, $n=61-69$

Insgesamt kann als Zwischenfazit festgestellt werden, dass für die Premiumstufe nur nach einer Aufklärung über die Bedeutung der Label (Fallstudie 1) eine höhere ZB besteht als für die Produkte der Einstiegsstufe. Die Unterschiede in der ZB bei Fallstudie 2 erweisen sich als nicht signifikant, sodass sich keine systematisch höhere Preisbereitschaft für die Premiumstufe erkennen lässt.

\section{$5 \quad$ Diskussion und Schlussfolgerung}

Die vorgestellten Fallstudien verdeutlichen, dass Verbrauchern allein anhand der Gestaltung die Bedeutung der unterschiedlichen Labelstufen nicht klar wird. Zwar assoziieren rund $16 \%$ der Verbraucher ungestützt mit der graphischen Gestaltung und spezifisch der Abbildung der beiden Sterne ein mehrstufiges kategoriales System, aber ohne eine weitere Aufklärung über die verschiedenen Level wird die Stufigkeit nicht erkannt. Dies spiegelt sich in den analysierten Zahlungsbereitschaften wider.

Während die ZB für die Tierschutz-Produkte mit Aufklärung über das Label (Fallstudie 1) für beide Stufen deutlich voneinander abweichen (die Premiumprodukte generieren eine höhere ZB als die Einstiegsprodukte), werden in der zweiten Fallstudie - ohne Aufklärung - relativ 
ähnliche ZB für die beiden Stufen angegeben. Lediglich für den Nussschinken ist die ZB für die zweite Stufe 4,9 Prozentpunkte höher als für das Produkt der Einstiegsstufe.

Für die Befragung mit Aufklärung ergeben sich wesentlich deutlichere Preisabstände zwischen den Labelstufen: Im Fall des Bacons sind die Probanden bereit, für das Premiumprodukt einen zusätzlichen Aufschlag von 21,8 Prozentpunkten gegenüber der Einstiegsstufe zu zahlen. Für das Premium-Nackensteak besteht sogar eine um 27,3 Prozentpunkte höhere ZB. Durch die Aufklärung über die Stufigkeit des Labels wurde demnach eine deutlich höhere ZB für die Premiumprodukte erzielt. Als Begründung für die höhere ZB im Falle des unverarbeiteten Produkts Nackensteak kann die größere Nähe des Produktes zum Tier vermutet werden.

Die in der ersten Fallstudie durchschnittlich akzeptierten Preisaufschläge für die Premiumprodukte verdeutlichen, dass die erhöhten Kosten für die Premiumstufe gegenüber der Einstiegsstufe durch die erzielten Preisaufschläge zumindest teilweise abgedeckt werden könnten. Die unsystematischen Werte in der zweiten Fallstudie verdeutlichen dagegen die Wichtigkeit der Verwendung von Kommunikationsmaterialien, um die Stufigkeit sowie deren Hintergründe und Folgen zu vermitteln. Die Preisaufschläge unterscheiden sich bei der zweiten Studie zwischen der ersten und der zweiten Stufe nicht signifikant, sodass hier die Mehrkosten für das Premiumsegment keinesfalls abgedeckt sind. Um den Verbrauchern zu kommunizieren, warum sie für die Premiumstufe mehr zahlen sollen als für Produkte der Einstiegsstufe, muss ihnen dies bspw. durch die Erläuterung zu den unterschiedlich strengen Vorgaben der beiden Labelstufen, den sich ergebenden Vorteilen für die Tiere und den daraus resultierenden höheren Kosten, die vor allem dem Landwirt entstehen, vermittelt werden. Nur so kann das zweistufige Labelsystem funktionieren und den deutlichen Mehrpreis für die Premiumstufe gegenüber den Verbrauchern rechtfertigen, damit diese die erforderliche höhere ZB für die Premiumprodukte akzeptieren und die Kosten so gedeckt werden können.

Hinzu kommt ein weiteres Problem: Die erste Studie zeigt, dass das Vertrauen in das Label relativ niedrig ist: Nur $35 \%$ der Teilnehmer halten das Label ohne Erläuterung für vertrauensvoll und nur $23 \%$ halten es für glaubwürdig. Nach einer Erläuterung zum Label erhöht sich der Anteil der Probanden, die Vertrauen in das Label haben, auf $63 \%$, und $40 \%$ halten nun das Label für glaubwürdig. Eine Etablierung des Labels ist daher nur möglich, wenn das basale Vertrauensdefizit beseitigt wird und auch dem Zeichengeber ein größeres Vertrauen entgegengebracht wird. Die generelle Kaufbereitschaft für Tierschutz-Produkte ebnet jedoch den Weg einer erfolgreichen Etablierung eines Tierschutzlabels: In der ersten Befragung sind 
$80 \%$ bereit, Tierschutzfleisch zu kaufen, nach der Aufklärung über das Label sogar $87 \%$. Bei der zweiten Befragung liegt der Anteil mit 78,3\% ebenfalls sehr hoch.

\section{$6 \quad$ Ausblick und Limitationen}

Die zweite, hier vorgestellte Studie ohne Aufklärung über die Bedeutung der Qualitätssymbole lässt erkennen, dass die Premiumstufe eines mehrstufigen Systems zumindest in der Einführungsphase ohne ausreichende Kommunikation auf Eintrittsbarrieren stoßen kann. Möglicherweise reicht die ZB dann nicht aus, um die beachtlichen Mehrkosten der zweiten Stufe zu decken. Entsprechende Erfahrungen werden aus dem bereits etwas länger etablierten niederländischen Tierschutzlabel berichtet, wo das 2-Sterne-Segment kaum akzeptiert wird (31).

Die Studie kann mangels detaillierter Kostendaten nicht ermitteln, ob der 1-Sterne-Standard für die Anbieter rentabel ist. Hierzu müssten neben den oben ansatzweise präsentierten landwirtschaftlichen Kosten auch Daten zu den Trennungs- und Vermarktungskosten für Industrie und Handel erhoben werden. Auch kann die Frage nicht beantwortet werden, ob bei hinreichender Aufklärung der Konsumenten (siehe Studie 1) das 1- oder das 2-Sterne-Segment rentabler ist. Solche Studien sollten zudem tierartenspezifisch, zunächst für die Haltungssysteme Schwein und Huhn, durchgeführt werden, da hier die Entwicklung der Labelstandards am weitesten ausgereift ist.

Es wird sich zeigen, ob sich ein Tierschutzlabel am Markt durchsetzen kann. Langfristig wird die Etablierung des Labels auf dem deutschen Markt schwierig werden, wenn es nicht gelingt, Kuppelprodukte (Wurst) zu einem höheren Preis zu verkaufen. Bislang wird relativ wenig Wurst als Tierschutz-Wurst vermarktet, ein Großteil wird als konventionelle Wurst zu einem niedrigeren Preis vertrieben. Der Absatz der Kuppelprodukte zu einem höheren Preis wird entscheidend sein, um die Kosten in der Wertschöpfungskette insgesamt geringer zu halten. Die Zahlungsbereitschaftsanalyse hat für verarbeitete Produkte erheblich niedrigere Werte aufgezeigt, sodass der Aufpreis nicht zu hoch sein darf, da sonst das Käufersegment deutlich kleiner wird (vgl. 20).

Weiterhin ist es wichtig, Landwirte für die Premiumstufe zu gewinnen, um auch eine größere Menge an entsprechenden Produkten vertreiben zu können und so die Nachfrage decken zu können, was wiederum auch den Erfolg des Labels beeinflusst.

Wie wichtig die Kommunikation bei der Einführung eines Labels ist, konnte in den Fallstudien gezeigt werden. Insgesamt ist der Zeichengeber (im Fallbespiel der Deutsche Tierschutzbund) gefordert, die Unterschiede zwischen den Standards im Labeldesign und durch Kom- 
munikation so zu gestalten, dass die Kosten der jeweiligen Anforderungen zum Ausdruck gebracht werden. Nur so können die entsprechenden notwendigen Zahlungsbereitschaften beim Verbraucher realisiert werden.

Grenzen der Studie sind weiterhin, dass es sich bei der abgefragten ZB um die hypothetische und nicht um die realisierte ZB handelt. Zwar wurde in der zweiten Studie ein Cheap-TalkSkript zur Vermeidung des hypothetical bias genutzt, gleichwohl ist nicht gewährleistet, dass die Probanden die angegebene ZB auch tatsächlich im Supermarkt umsetzen würden. In einem Marktexperiment oder in einem Testsupermarkt, der zusätzlich die Kontrolle von Störfaktoren erleichtert, ließe sich die tatsächliche ZB genauer testen. Schließlich ist die Vergleichbarkeit der Studien zusätzlich zu den unterschiedlichen Informationen noch dadurch gemindert, dass in der Befragung mit Aufklärung allen Probanden Produkte der Einstiegsund der Premiumstufe vorlagen, während die Teilnehmer der Studie ohne Aufklärung entweder Produkte der Einstiegs- oder der Premiumstufe vorliegen hatten.

\section{Zusammenfassung}

Die landwirtschaftliche Nutztierhaltung ist ein aktuelles und kontrovers diskutiertes Thema. Besonders aus Verbrauchersicht ist die Thematik negativ besetzt und wird oft unter dem Begriff „Massentierhaltung“ gerahmt. Tatsache ist, dass die Haltungsbedingungen und damit das Tierwohl sich innerhalb der einzelnen Betriebsformen und Betriebe stark unterscheiden. Neben der konventionellen und der ökologischen Tierhaltung entwickelt sich derzeit im Markt eine Zwischenstufe: Ein Haltungssystem, dessen Anforderungen über den gesetzlichen, aber unter den vorgeschriebenen ökologischen Standards liegt. Um diese bestehenden Unterschiede auch innerhalb eines Zertifizierungssystems für Verbraucher sichtbar zu machen, kann ein mehrstufiges Labellingsystem eine Lösung sein. Ein höheres Maß an Tierwohl ist dabei mit höheren Kosten verbunden. Im vorliegenden Beitrag werden daher an Hand von zwei Fallstudien Zahlungsbereitschaften deutscher Verbraucher für ein mehrstufiges Tierschutzlabel analysiert. Es zeigt sich, dass die entstehenden Mehrkosten der Premiumstufe ohne vertiefte Informationen nicht durch die ermittelten Zahlungsbereitschaften abgedeckt werden können. Um die Zahlungsbereitschaft zu erhöhen, kann eine verbesserte Kommunikationsstrategie, die über die Unterschiede informiert, eine Lösung sein. 
Summary: Willingness to pay analyses in multi-level labelling systems: The animal welfare label example

Livestock farming is a current and controversially discussed topic. Especially from the consumer's point of view, the topic has a negative connotation and is often discussed using the term "factory farming". Animal welfare conditions differ greatly within single farming companies. Besides conventional and organic livestock farming there is an intermediate stage developing in the market: A husbandry system whose requirements are higher than the legal ones but lower than organic standards. A multi-level labelling system could possibly be a solution to uncovering, for consumers, the existing differences within a certification system. A higher degree of animal welfare is associated with higher costs. Therefore, in the present paper two case studies analyze the willingness to pay of German consumers for a multi-level animal welfare label. It is shown that the willingnesses to pay do not cover the additional costs for the premium level without in-depth information. To increase the willingness to pay, an improved communication strategy could potentially be a solution.

Résumé : Analyse de disponibilité de consommateurs à payer pour des labels successifs: Prenant pour l'exemple le label bien-être des animaux

La production agricole est un sujet d'actualité et prête à des discussions controversées. Particulièrement du point vue du consommateur ce sujet a une connotation négative et les discussions sont encadrés par la notion ,élevage / production de masse d'animaux“. Le fait est que les conditions d'élevage et par conséquent le bien-être des animaux différent fortement par rapport au mode et forme d'exploitation agricole. Outre l'élevage d'animaux conventionnels ou biologiques le marché développe également un mode d'élevage intermédiaire: ayant des standards plus élevés que ceux requis par la loi mais inférieurs aux standards biologiques. Pour rendre ces différences visibles aux consommateurs un label avec plusieurs niveaux pourrait être une solution. Cela implique qu'un niveau plus élevé de bien-être animal est lié à des dépenses plus élevés. A l'aide de deux études modèles cet article analyse la disponibilité du consommateur allemand à payer pour un label successif de bien-être animal. On constate que les surcoûts pour l'élevage respectant les plus hauts standards de bien-être des animaux ne peuvent pas être couverts par la disposition des consommateurs analysés à payer plus sans informations approfondies. Pour augmenter leur disposition à payer une meilleure stratégie de communication qui informe sur les raisons des surcouts pourrait être une solution. 


\section{Literatur}

1. Abrate, G., Capriello, A., Fraquelli, G., 2011: When quality signals talk: Evidence from the Turin hotel industry. In: Tourism Management, 32 (4), S. 912-921.

2. ACHILles, F., Fritzsche, S., 2014: Kosten und Nutzen eines Tierschutzlabels in der Schweinemast. In: Landtechnik, 68 (4), S. 235-241.

3. Agrarmarkt Informations-Gesellschaft $\mathrm{mbH}$, 2013: Marktdaten Ökolandbau. URL: http://www.ami-informiert.de/ami-onlinedienste/serviceportal-forschunglehre/oekolandbau/marktdaten-download.html. Abrufdatum: 19.06.2013.

4. Agrarmarkt Informations-Gesellschaft mbH, 2014: EU-Schweinepreise erreichen 2013 ein Rekordniveau. URL: http://www.ami-informiert.de/ami-maerkte/ami-weiteremaerkte/ami-maerkte-agrarwirtschaft/meldungen/meldungen-single-ansicht/article/euschweinepreise-erreichten-2013-rekordniveau.html. Abrufdatum: 07.07.2014.

5. Akaichi, F., Revoredo-Giha, C., Leat, P., ARnoult, M., 2013: Consumer Demand for Products with Animal Welfare Attributes. In: Labels on sustainability: an issue for consumers, producers, policy makers and NGOs. Paris.

6. Bennett, R., Thompson, P., 2011: Economics. In: Appleby, M. C., Mench, J. A., Olsson, I. A. S., Hughes, B. O. (Hrsg.). Animal Welfare. 2nd ed., S. 279-290. Wallingford: Centre for Agricultural Bioscience International.

7. Beukert, C., Simons, J., 2006: Der Markt für ökologisch erzeugte Fleischprodukte: Wachstumsimpulse durch den Aufbau einer effizienten und konsumtenorientierten Wertschöpfungskette, No. 135. Bonn.

8. Busch, G., KAYSER, M., SpILler, A., 2012: Massentierhaltung aus Vebrauchersicht Assoziationen und Einstellungen. In: Ökodienstleistungen und Landwirtschaft: Herausforderungen und Konsequenzen für Foschung und Praxis, S. 123-124. Wien: 22. Jahrestagung der Österreichischen Gesellschaft für Agrarökonomie.

9. DEHOGA Bundesverband, 2012a: 5 gute Gründe für die deutsche Hotelklassifizierung. URL: http://www.hotelsterne.de/de/?open=Gute_Gruende. Abrufdatum: 08.12.2012

10. DEHOGA Bundesverband, 2012b: Deutsche Hotelklassifizierung. URL: http://www.dehoga-bundesverband.de/. Abrufdatum: 08.12.2012.

11. Deimel, I., Franz, A., Frentrup, M., von Meyer, M., Spiller, A., Theuvsen, L., 2010: Perspektiven für ein Europäisches Tierschutzlabel. URL: download.ble.de/08HS010.pdf. Abrufdatum: 30.11.2012.

12. Deutsche Energie Agentur, 2012: EU-Label: Energieeffizienzsteigerung durch Energieverbrauchskennzeichnung.

URL: http://www.dena.de/fileadmin/user_upload/Nachrichten/2012/Factsheet_EU_Label.pdf. Abrufdatum: 02.05.2013

13. Deutscher Tierschutzbund e. V., 2013a: Entwicklung des Tierschutzlabels. URL: http://www.tierschutzlabel.info/wissenschaft/entwicklung/. Abrufdatum: 30.03.2013.

14. Deutscher Tierschutzbund e. V., 2013b: Kriterienkataloge. URL: http://www.tierschutzlabel.info/tierschutzlabel/. Abrufdatum: 07.07.2014.

15. ElbakidZE, L., NAYGA, R. M. J., LI, H., 2012: Willingness to Pay for Multiple Quantities of Animal Welfare Dairy Products: Results from Random Nth-, Second-Price, and Incremental Second-Price Auctions. In: Canadian Journal of Agricultural Economics/Revue Canadienne D'agroeconomie. doi:10.1111/j.1744-7976.2012.01263.x.

16. European Commission, 2005: Attitudes of consumers towards the welfare of farmed animals. Special Eurobarometer 229 / Wave 63.2.

17. European Commission, 2006: Labelling: competitiveness, consumer information and better regulation for the EU. URL: http://ec.europa.eu/food/food/labellingnutrition /betterregulation/competitiveness_consumer_info.pdf. Abrufdatum: 16.04.2014. 
18. European Commission., 2007: Attitudes of EU citizens towards Animal Welfare. Special Eurobarometer 270 / Wave 66.1.

19. FARrell, J., RABIN, M., 2013: Cheap Talk. In: The Journal of Economic Perspectives, 10 (3), S. 103-118.

20. GRAnNis, B. J., Thilmany, D., 2001: Marketing Opportunities for Natural Pork Products : Defining the Consumers Who Would Purchase Natural Pork. In: Journal of the American Society of Farm Managers and Rural Appraisers, S. 93-98.

21. Grimsrud, K. M., Nielsen, H. M., NAVRud, S., Olesen, I., 2013: Households' willingness-to-pay for improved fish welfare in breeding programs for farmed Atlantic salmon. In: Aquaculture, 372-375, S. 19-27.

22. Harper, G. C., MaKatouni, A., 2002: Consumer perception of organic food production and farm animal welfare. In: British Food Journal, 104 (3), S. 287-299. doi:10.1108/00070700210425723.

23. HeInZle, S., WÜSTEnHAGEN, R., 2010: Disimproving the European Energy Label's value for consumers? - Results of a consumer survey. St. Gallen.

24. Hempler, J. (2014): Drei Programme im Vergleich. URL: http://www.neulandfleisch.de/assets/files/Pressespiegel/2014/DLG3-2014.pdf. Abrufdatum: 07.07.2014.

25. Hoste, R., 2010: Economische gevolgen van meer leefoppervlakte voor vleesvarkens: Economische gevolgen van meer leefoppervlakte voor vleesvarkens, No. 2010-012. Den Haag.

26. Kehlbacher, A., Bennett, R., Balcombe, K., 2012: Measuring the consumer benefits of improving farm animal welfare to inform welfare labelling. In: Food Policy, 37 (6), S. 627-633. doi:10.1016/j.foodpol.2012.07.002

27. LAGerkVist, C. J., HeSs, S., 2010: A meta-analysis of consumer willingness to pay for farm animal welfare. In: European Review of Agricultural Economics, 38 (1), S. 55-78. doi:10.1093/erae/jbq043

28. LeE, T.-R., Lin, C.-H., DaduRA, A. M., Genshi, K., 2012: Promotion of economic animal welfare by market force: a case study. In: International Journal of Business Innovation and Research, 6 (3), S. 302-321.

29. LÜTH, M., SPILLER, A., 2003: Qualitätssignaling in der Gastronomie, No. 0301. Göttingen.

30. Miller, G. Y., Unnevehr, L. J., 2001: Characteristics of Consumers Demanding and Their Willingness to Pay for Certified Safer Pork. In: Journal of Agribusiness, 19 (2), S. 101-119.

31. Ministerie van Economische Zaken, 2012: Monitor Duurzaam Voedsel 2012. Consumentenbestedingen aan duurzaam gelabelde producten. URL: file:///C:/Users/rwe.AGCC/Downloads/monitor-duurzaam-voedsel-2012.pdf. Abrufdatum: 07.07.2014.

32. Nocella, G., Hubbard, L., Scarpa, R., 2010: Farm Animal Welfare, Consumer Willingness to Pay, and Trust: Results of a Cross-National Survey. In: Applied Economic Perspectives and Policy, 32 (2), S. 275-297. doi:10.1093/aepp/ppp009

33. Norwood, F. B., LusK, J. L., 2011: Compassion, by the Pound:The Economics of Farm Animal Welfare. Oxford: Oxford University Press.

34. SCHÄFER, M., 2003: Der Geist ist willig, alleine das Fleisch ist schwach... Motivationen für die Ernährung mit Bioprodukten und der Zusammenhang mit umweltfreundlichem Handeln. In: AgrarBündnis AG Land- und Regionalentwicklung und Universität \& Kassel (Hrsg.), Der kritische Agrarbericht. Jahrbuch, S. 276-277. Hamm: AbL Bauernblatt Verlags-GmbH.

35. SCHRÖCK, R., 2013: Analyse der Preiselastizitäten der Nachfrage nach Biolebensmitteln unter Berücksichtigung nicht direkt preisrelevanten Verhaltens der Verbraucher. Abschlussbericht des BÖLN Projekts 2808 OE 148. Gießen. 
36. Schulze, B., SpIller, A., Lemke, D., 2008: Glücksschwein oder arme Sau? Die Einstellung der Verbaucher zur modernen Nutztierhaltung. In Spiller, A., Schulze, B. (Hrsg.): Zukunftsperspektiven der Fleischwirtschaft - Verbraucher, Märkte Geschäftsbeziehungen, S. 465-489. Göttingen: Universitätsverlag Göttingen.

37. SimONS, J., HARMANN, M., 2007: Klare Vorstellungen fehlen weitgehend. Bio-Fleisch im Spannungsfeld zwischen Exklusivität und Banalität - Teil 1: Der Markt. In: Fleischwirtschaft, 2, S. 56-60.

38. Simons, J., Harmann, M., Beukert, C., 2007: Bio-Fleisch im Spannungsfeld zwischen Exklusivität und Banalität - Teil 2: Die Wertschöpfungskette. In: Fleischwirtschaft, 3, S. 85-92.

39. Snyder, W., Cotter, M., 1998: The Michelin Guide and Restaurant Pricing Strategies. In: Journal of Restaurant \& Foodservice Marketing, 3 (1), S. 51-67.

40. SPILlER, A., SCHUlzE, B., 2008: Zukunftsperspektiven der Fleischwirtschaft. Göttingen: Universitätsverlag Göttingen.

41. Statistisches Bundesamt, 2011: Statistisches Jahrbuch 2011. Wiesbaden. URL: https://www.destatis.de/DE/Publikationen/StatistischesJahrbuch/StatistischesJahrbuch201 1.pdf?_blob=publicationFile. Abrufdatum: 16.04.2014.

42. Stolz, H., 2009: Warum deutsche Verbraucher Einzelmerkmale des Öko-Landbaus stärker bevorzugen als das Gesamtsystem Öko-Landbau. In: Mayer, J., Alföldi, T., Leiber, F., Dubois, D., Fried, P., Heckendorn, F., ... Willer, H. (Hrsg.): Werte - Wege Wirkungen: Biolandbau im Spannungsfeld zwischen Ernährungssicherung, Markt und Klimawandel. Beiträge zur 10. Wissenschaftstagung Ökologischer Landbau, ETH Zürich, 11.-13. Februar 2009. Band 2: Tierhaltung, Agrarpolitik und Betriebswirtschaft, S. 336340. Berlin: Verlag Dr. Köster.

43. Thelen, E., Botschen, M., 2005: Warum Bio? - Einstellungen zum Konsum von Bioprodukten in Österreich. In: Groier, M. Schermer, M. (Hrsg.), Bio-Landbau in Österreich im internationalen Kontext, S. 53-67. Wien.

44. Truffler, B., Markard, J., Wüstenhagen, R., 2001: Eco-labeling of electricity strategies and tradeoffs in the definition of environmental standards. In: Energy Policy, 29, S. 885-897.

45. ZANDER, K., HAMM, U., 2009: Informationsverhalten der Konsumenten und ethische Werte ökologischer Lebensmittel Methode Ergebnisse und Diskussion. In: Mayer, J., Alföldi, T., Leiber, F., Dubois, D., Fried, P., Heckendorn, F., ... Willer, H. (Hrsg.): Werte - Wege - Wirkungen: Biolandbau im Spannungsfeld zwischen Ernährungssicherung, Markt und Klimawandel. Beiträge zur 10. Wissenschaftstagung Ökologischer Landbau, ETH Zürich, 11.-13. Februar 2009. Band 2: Tierhaltung, Agrarpolitik und Betriebswirtschaft, S. 340-342. Verlag Dr. Köster.

\section{Dank}

Die Veröffentlichung wurde im Projekt "Tierwohllabel - Aufbau eines marktgerechten Tierwohlprogramms in der Schweinefleischkette" durch das Bundesministerium für Ernährung und Landwirtschaft und die Bundesanstalt für Landwirtschaft und Ernährung gefördert. 


\section{Teil II: Consumer understanding of food packaging}

II.1 Verbraucherverständnis von Verkehrsbezeichnungen

Autoren: Ramona Weinrich, Sina Nitzko, Achim Spiller, Anke Zühlsdorf

Veröffentlicht bei Journal für Verbraucherschutz und Lebensmittelsicherheit, 2015: 10 (1), 13-22, with permisson of Springer 


\section{Zusammenfassung}

Viele Verbraucher interessieren sich für die Qualität von Lebensmitteln und deren Eigenschaften und Zusammensetzung. Eine wichtige Schlüsselinformation über die Beschaffenheit eines Produktes kann für Verbraucher die Verkehrsbezeichnung auf Fertigverpackungen von Lebensmitteln sein, deren Angabe gesetzlich verpflichtend ist. Die Verkehrsbezeichnung, d. h. die kurze Charakterisierung der Produktart, zielt auf die eindeutige Benennung eines Produktes für den Verbraucher ab, sodass dieser das Erzeugnis von anderen unterscheiden kann. Welche Verkehrsbezeichnung zu verwenden ist, ist für einige Produkte gesetzlich festgeschrieben. In anderen Fällen kann auf die allgemeine Verkehrsauffassung Bezug genommen oder eine beschreibende Bezeichnung genutzt werden. Bei zahlreichen Lebensmitteln kommt es zwischen Herstellern und Verbrauchervertretern zu Konflikten um die Frage, ob die verwendeten Bezeichnungen der Verbrauchererwartung entsprechen bzw. irreführend wirken. Mittels einer Verbraucherbefragung (repräsentativ für Alter, Geschlecht, Einkommen, regionale Verteilung, Bildung und Haushaltsgröße in Deutschland, $n=1.021$ ) wird überprüft, ob das Begriffsverständnis der Konsumenten mit der tatsächlichen Beschaffenheit von drei Beispielprodukten übereinstimmt. Anhand der Ergebnisse der Studie lässt sich aufzeigen, dass Verbraucher die Lebensmittelkennzeichnungen teilweise anders verstehen, als es der tatsächlichen Beschaffenheit des Produktes entspricht. Auch werden veränderte Verarbeitungstechnologien nicht immer schnell genug in die Regelung zur Verkehrsbezeichnung übernommen.

Schlüsselwörter Lebensmittelkennzeichnung, Verkehrsbezeichnung, Verbrauchertäuschung, Irreführung, Verkehrsauffassung, Konsumentenverhalten

\section{Einleitung}

Heutzutage sieht sich der Verbraucher einer großen Auswahl an Produkten im Lebensmitteleinzelhandel gegenüber. Dabei nimmt die Zeit, die er zum Einkaufen verwendet, tendenziell ab (Schrankel 2005). Das Resultat ist eine Verschärfung der von der Marketingforschung seit geraumer Zeit konstatierten Informationsüberlastung (Kolodinsky 2012; Kroeber-Riel und Esch 2004; van Kleef et al. 2008).

Umso wichtiger ist es für Verbraucher, dass sie sich auf Schlüsselelemente einer Lebensmittelverpackung verlassen können. Jedes Lebensmittel, das in einer Fertigverpackung vertrieben wird, muss z. B. mit einer Verkehrsbezeichnung versehen sein ( 3 Abs. 1 S. 1 LMKV). Die 
Verkehrsbezeichnung soll ermöglichen, die Art eines Produktes auf den ersten Blick zu identifizieren ( $\$ 4$ Abs. 1 S. 2 LMKV).

Konkrete Verkehrsbezeichnungen werden für einige Produkte in spezifischen Produktverordnungen gesetzlich vorgegeben. Teilweise sind sie in den Leitsätzen des Deutschen Lebensmittelbuchs beschrieben oder können durch die Hersteller selbst festgelegt werden, was zu Konflikten führen kann (z. B. LMRR 1984, 38; LMRR 1989, 59; LMRR 1999, 132; LMRR 2012, 53). In anderen Fällen sind auch gesetzlich festgelegte Verkehrsbezeichnungen unklar geregelt und stoßen auf Protest (z. B. LMRR 2001, 76; LMRR 2010, 72; LMRR 2011, 95). Für einen funktionierenden Markt ist es aber wichtig, dass ausgelobte Produkteigenschaften klar und deutlich in korrekter Form kommuniziert werden und sich hinter derselben Verkehrsbezeichnung dieselben Zusammensetzungen und Inhaltsstoffe verbergen. Nur so kann gewährleistet werden, dass keine Verdrängung lauterer Wettbewerber bzw. Produkte von besseren Qualitäten aus dem Markt stattfindet (Akerlof 1970).

In dem vorliegenden Artikel soll geklärt werden, inwiefern das Verbraucherverständnis bei ausgewählten Verkehrsbezeichnungen von der Kennzeichnungspraxis abweicht. Der Beitrag ist dabei wie folgt aufgebaut: Nach der Darlegung der Verbraucherwahrnehmungen von Produktinformationen auf Lebensmittelverpackungen wird aufgeführt, welche rechtlichen Grundlagen zur Verkehrsbezeichnung auf Lebensmitteln bestehen. Es werden Rechtsurteile herangezogen, welche verdeutlichen, wie Gerichte in Streitfragen mit der Beurteilung von Verkehrsbezeichnungen umgehen. Im Anschluss wird eine eigene Studie vorgestellt, die analysiert, wie bestimmte Verkehrsbezeichnungen von Verbrauchern verstanden werden. Als Fallbeispiele werden die Produkte Geflügelwurst, Kalbswurst und alkoholfreies Bier angeführt. Anschließend werden die Ergebnisse der Studie dargestellt und diskutiert. Die Produktbeispiele sollen aufzeigen, ob die derzeit geltenden Vorschriften der Lebensmittelkennzeichnung für Verkehrsbezeichnungen dem Verbraucherverständnis entsprechen.

\section{Verbraucherwahrnehmung von Produktinformationen}

Die Lebensmittelkennzeichnung gibt Verbrauchern wichtige Hinweise über die Beschaffenheit und Zusammensetzung des Produktes. Neben den Schwierigkeiten, die Verbraucher haben, die Informationen auf der Verpackung zu verstehen (Harper et al. 2007), erscheinen die Lebensmittelverpackungen oft mit Abbildungen, Markennamen, Labeln und Texten überladen (Orth und Malkewitz 2008). Dies macht es noch schwieriger für die Konsumenten, wichtige Informationen zu filtern. Das Resultat ist ein sogenannter ,information overload“ (Informationsüberlastung) (Kolodinsky 2012; Kroeber-Riel and Esch 2004; van Kleef et al., 2008). Die 
Aufnahmefähigkeit der Verbraucher begrenzt letztendlich die Fähigkeit, Informationen auf Verpackungen zu verarbeiten (Sørensen et al. 2012). Einige Konsumenten sind sogar durch die vielen Informationen auf Verpackungen gelangweilt und ignorieren sie (Iyengar et al. 2003; Iyengar und Lepper 2000). Die Platzierung von entscheidenden Informationen wie der Verkehrsbezeichnung auf der Produktvorderseite wird in diesem Zusammenhang als eine Möglichkeit diskutiert, die Beschaffenheit eines Produktes auf den ersten Blick erkennen zu können (Dantas 2004; van Herpen und van Trijp 2011; Zühlsdorf et al. 2013). In der Konsumentenforschung ist bisher allerdings nicht geklärt, wie Informationen auf einem Produkt auf Grund der derzeitigen Gesetzeslage von Konsumenten wahrgenommen und interpretiert werden.

\section{Rechtlicher Hintergrund}

Zum Schutz der Verbraucher vor irreführenden Kennzeichnungen und zur Reglementierung des Verhaltens der Anbieter untereinander wurden verschiedene Rechtsvorschriften und Verordnungen erlassen. Zunächst findet sich im Gesetz gegen den unlauteren Wettbewerb (UWG) ein allgemeines Irreführungsverbot. § 3 Absatz 1 UWG ist zu entnehmen, dass unlautere Geschäftshandlungen unzulässig sind, sobald sie die Interessen von Verbrauchern (oder Wettbewerbern) spürbar beeinträchtigen. Nach $\S 5$ Absatz 1 UWG handelt unlauter, „,wer eine irreführende geschäftliche Handlung vornimmt“. Entscheidend ist dabei, dass die Aufmachung eines Produkts geeignet ist, eine nicht unerhebliche Anzahl an Verbrauchern irrezuführen.

Im Lebensmittelrecht sind auf nationaler Ebene die $\S \S 11$ und 12 des Lebens- und Futtermittelgesetzbuches (LFGB) einschlägig. Hier wird auf den Gesundheitsschutz der Verbraucher abgestellt. In $§ 11$ sind die Vorschriften zum Schutz vor Täuschung geregelt. Nach Absatz 1 ist es verboten, Lebensmittel unter irreführender Bezeichnung, Angabe oder Aufmachung in den Verkehr zu bringen oder mit irreführenden Darstellungen oder Aussagen zu werben. Des Weiteren ist es nach Absatz 2 Satz 2 verboten, Lebensmittel, die in ihrer Beschaffenheit von der Verkehrsauffassung abweichen und dadurch in der Qualität nicht unerheblich gemindert sind, ohne Kenntlichmachung in den Verkehr zu bringen. Maßgeblich für eine Irrführungsgefahr ist die Verkehrsauffassung (Helm 2010, § 59 Rdnr. 67), d. h. die Einschätzung der angesprochenen Verkehrskreise (Sosnitza 2010, UWG § 5 Rn 114). Unter der Verkehrsauffassung werden zum einen die redliche Herstellerpraxis und zum anderen die Verbrauchererwartungen an das Produkt zusammengefasst (BMELV 2013a). 
Die Kennzeichnung von Lebensmitteln in Fertigverpackungen ist in Deutschland in der Lebensmittelkennzeichnungsverordnung (LMKV) geregelt. Diese setzt die EU-Richtlinie 2000/13/EG vom 20. März 2000 um. Nach § 3 Absatz 1 Satz 1 LMKV ist ein Lebensmittel in einer Fertigverpackung mit einer Verkehrsbezeichnung zu versehen. Insgesamt ergeben sich drei Möglichkeiten, nach denen die Verkehrsbezeichnung gewählt wird (Grube 1997):

i) Die Verkehrsbezeichnung ist in einer Rechtsvorschrift festgelegt. Damit ist die Verwendung dieser Bezeichnung rechtsbindend vorgeschrieben ( 4 Abs. 1 LMKV; vgl. auch Art. 2 Abs. 1 lit. n VO 1169/2011/EG).

ii) Es wird die verkehrsübliche Bezeichnung gewählt. Diese entspricht einer ,nach allgemeiner Verkehrsauffassung übliche[n] Bezeichnung“ (§ 4 Abs. 1 S. 1 LMKV; vgl. auch Art. 2 Abs. 1 lit. o VO 1169/2011/EG). Sie kann beispielsweise den Vorgaben

a. der Deutschen Lebensmittelbuch-Kommission oder

b. einem Wirtschaftsverband

entnommen werden.

iii) Ist weder eine rechtsverbindliche Verkehrsbezeichnung noch eine verkehrsübliche Bezeichnung für das Produkt vorhanden, so findet eine Beschreibung des Lebensmittels durch den Produzenten statt ( $\$ 4$ Abs. 1 S. 2 LMKV; vgl. auch Art. 2, Abs. 1 lit. p VO 1169/2011/EG).

Eine große praktische Bedeutung bei der Findung der Verkehrsauffassung hat die Deutsche Lebensmittelbuch-Kommission, die in den Leitsätzen des Deutschen Lebensmittelbuchs die Verkehrsbezeichnung vieler Produkte definiert. Diese Leitsätze haben den Charakter von objektivierten Sachverständigengutachten und sind keine bindenden Rechtsvorschriften. Nach $\S 15$ LFGB sind die Leitsätze vor allem für die Verkehrsfähigkeit von Lebensmitteln von Bedeutung. Die Leitsätze werden von der Lebensmittelbuch-Kommission erarbeitet, die paritätisch aus Vertretern der Wissenschaft, Lebensmittelwirtschaft, Verbraucherschaft und der Lebensmittelüberwachung zusammengesetzt ist. Das Deutsche Lebensmittelbuch beinhaltet Beschreibungen zu Herstellung, Beschaffenheit und Merkmalen von Lebensmitteln (BMELV 2013b).

\section{Feststellung der Verkehrsauffassung}

Bei gerichtlichen Entscheidungen über strittige Verkehrsbezeichnungen kommt der Feststellung der Verkehrsauffassung eine zentrale Rolle zu. Die Verkehrsauffassung ist ein Konstrukt, welches sowohl die Usancen der Hersteller und Händler (,redliche Herstellerpraxis“, 
„Handelsbrauch“) als auch das Verständnis der angesprochenen Verbraucher zusammenfasst und daraus den subjektiven (d. h. durch die Sicht der angesprochenen Verkehrskreise geprägten) Bedeutungsgehalt einer werblichen Aussage - hier der Verkehrsbezeichnung - ableitet. Im Zweifel, wenn sich Position der Anbieter und Erwartungen der Nachfrager widersprechen, kommt dabei der Verbrauchererwartung Priorität zu (Hohmann 1994, 382; Hecker 2001).

Da die Richtlinien zur Festlegung der Verkehrsbezeichnung Gestaltungsräume offen lassen, setzen Hersteller die Vorgaben teilweise unterschiedlich um (vgl. Kapitel 6). Diese rechtlichen Grauzonen führen dazu, dass bei Unklarheiten über die Richtigkeit bzw. Verständlichkeit der Verkehrsbezeichnung in zunehmendem Maße Gerichte angerufen werden, um Streitfragen zwischen Wettbewerbern oder zwischen einem Hersteller und Verbrauchern zu klären.

Bei der Feststellung der Verkehrsauffassung kommen empirische Erhebungen heute nach gängiger Praxis nur in Ausnahmefällen zum Zuge. In einem Urteil aus dem Jahre 1969 legt der BGH dar, dass der Richter unabhängig von Sachverständigen eine Entscheidung treffen kann, wenn er selbst über die notwendige Sachkunde verfügt (GRUR 1970, 462, 464 - EuroSpirituosen). Generell kann ein Richter auf Grund seiner eigenen Sachkunde ein Urteil treffen, wenn Folgendes zutrifft:

i) Es muss sich um Waren des täglichen Bedarfs handeln.

ii) Der Richter muss Teil des angesprochenen Verkehrskreises sein.

iii) Es dürfen keine Umstände vorliegen, die Zweifel an der Sachkunde des Richters aufkommen lassen (Tilmann 1984, 716f).

In Zweifelsfällen ist die empirische Sozialforschung zur Feststellung der Verkehrsauffassung der Verbraucher jedoch zulässig. Entscheidend ist, dass es um die Verkehrsauffassung im Sinne des Verbraucherverständnisses geht (Sosnitza 2010, UWG $§ 2$ Rdnr. 95). Bei der Anrufung des EuGH durch ein deutsches Gericht verwies dieser darauf, dass bei besonderen Schwierigkeiten bei der Beurteilung der Irreführungsgefahr das nationale Gericht ein Sachverständigengutachten oder eine Verbraucherbefragung zur Ermittlung heranziehen kann (GRUR Int. 1998, 295, 296, 297). In der Praxis werden Verbraucherbefragungen zumeist von Wettbewerbern erhoben, wenn der Richter dazu neigt, die Irreführungsgefahr zu verneinen (vgl. dazu BGH GRUR 1963, 270 - Bärenfang). In der Literatur wird darüber hinaus grundsätzlich diskutiert, ob ein Richter noch unvoreingenommen sein kann, wenn er durch die ausführliche Darlegung des Falls stark involviert ist und daher über deutlich mehr Informationen als ein Verbraucher vor einem Regal im Supermarkt verfügt (Deutsch 1996). 
Mögliche Gründe für die geringe Relevanz der demoskopischen Empirie bei Gericht liegen in den hohen Kosten, die mit einer repräsentativen Befragung verbunden sind, aber auch im Zeitaufwand für Konzeption und Durchführung. Bedeutend dürfte zudem die Entscheidungshoheit der Gerichte sein. Die Richter folgen meist der Auffassung, dass eine normative Bewertung zur sachgerechten Entscheidungsfindung der richtige Weg ist (Pflüger 2010, § 42 Rdnr. 5ff). Ein weiterer wichtiger Grund, warum Gerichte selten Sachverständigengutachten in Form von Meinungsumfragen zu Rate ziehen, sind Zweifel an der Methodik, d. h. die Gefahr einer suggestiven Fragestellung und einer nicht objektiven Betrachtungsweise bei der Fragebogenerstellung und der Befragung (Köhler und Bornkamm 2013, UWG $\S 12$ Rdnr. 2.78-2.85).

Insgesamt zeigt sich damit, dass die juristische Überprüfung der Verkehrsauffassung der Verkehrsbezeichnung mittels empirischer Untersuchungen selten ist. Auch ansonsten wurden zu dieser Thematik in der Konsumforschung u. W. bisher keine Studien durchgeführt. Gleichzeitig gibt es zu einer Reihe von Verkehrsbezeichnungen derzeit eine lebhafte öffentliche Diskussion, z. B. im Rahmen des von den Verbraucherzentralen realisierten Internetportals Lebensmittelklarheit.de. Die folgende Befragung soll vor diesem Hintergrund weiterführende Hinweise liefern, ob Verbraucher die Verkehrsbezeichnungen so auffassen, wie es das $\mathrm{Zu}$ tatenverzeichnis zu verstehen gibt.

\section{Material und Methoden}

In einer eigenen Studie wurde untersucht, wie Lebensmittelkennzeichnungen und Produktaufmachungen vom Verbraucher verstanden werden. Die Befragung ist im Rahmen der begleitenden Verbraucherforschung zum Internetportal Lebensmittelklarheit.de der Verbraucherzentralen und des Verbraucherzentrale Bundesverbands durchgeführt worden (BMELV 2013c $)^{4}$. Der hier präsentierten quantitativen Studie waren Fokusgruppendiskussionen zur Festlegung der Befragungsthemen und zur Hypothesengenerierung vorangestellt (Zühlsdorf et al. 2013).

Die Verbraucherbefragung wurde als standardisierte computergestützte persönliche Befragung (CAPI-Interviews) mit 1.021 Teilnehmern mit Hilfe eines führenden Marktforschungsunternehmens durchgeführt. Die Studienteilnehmer waren deutsche Verbraucherinnen und Verbraucher ab 16 Jahren. Die Befragung fand im November und Dezember 2012 statt. Um

\footnotetext{
${ }^{4}$ Das Projekt wurde im Rahmen der Initiative „Klarheit und Wahrheit bei der Kennzeichnung und Aufmachung von Lebensmitteln“ vom Bundesministerium für Ernährung und Landwirtschaft gefördert.
} 
Reihenfolgeneffekte auszuschließen, wurden die Items strikt randomisiert. Die Auswertung erfolgte mit dem Statistikprogramm IBM ${ }^{\circledR}$ SPSS, Version 20, anhand univariater (Mittelwerte und Standardabweichungen) und bivariater (ANOVA, Korrelation und Kreuztabellen) Statistik.

Um eine Repräsentativität für die deutsche Bevölkerung zu gewährleisten, wurden Quoten für die Merkmale Alter, Geschlecht, Einkommen, regionale Distribution, Bildung und Haushaltsgröße entsprechend der Verteilung gesetzt. Die detaillierten soziodemografischen Merkmale sind Tabelle 1 des Anhangs zu entnehmen. Die Quotenvorgaben wurden erfüllt. Somit kann die Stichprobe als repräsentativ für die Bevölkerung in der Bundesrepublik Deutschland angesehen werden (Statistisches Bundesamt 2012).

Ergänzt werden die Darstellungen der Fallstudien durch eigene Recherchen zur Produktzusammensetzung der Produkte unterschiedlicher Hersteller ${ }^{5}$.

\section{Analysen der Fallbeispiele}

Im Folgenden werden drei Produkte (Geflügelwurst, Kalbswurst, alkoholfreies Bier) als Fallbeispiele für das Verbraucherverständnis von Verkehrsbezeichnungen herangezogen. Diese Erzeugnisse wurden ausgewählt, da sie in der Vergangenheit mehrfach in der (Fach)Öffentlichkeit kontrovers diskutiert wurden (z. B. Stiftung Warentest 2011).

Für die Produkte Geflügelwurst und Kalbswurst sind die Leitsätze für Fleisch und Fleischerzeugnisse relevant. Für alkoholfreies Bier können verkehrsübliche Bezeichnungen des Wirtschaftsverbands Deutscher Brauer-Bund herangezogen werden. Der Deutsche Brauer-Bund bezieht sich in Teilen wiederum auf die Weinverordnung (WeinV). Tabelle 1 stellt die relevanten Quellen zusammenfassend dar.

\footnotetext{
${ }^{5}$ Die Recherchen zu den Produktzusammensetzungen fanden im Frühjahr 2013 statt.
} 
Tabelle 1 Quellen für die Festlegung der Verkehrsbezeichnungen der Fallbeispiele

\begin{tabular}{l|l} 
Fallbeispiel & Lebensmittelrechtlich relevante Quelle \\
\hline Geflügelwurst & $\begin{array}{l}\text { Leitsätze für Fleisch und Fleischerzeugnisse 2.11: Wenn außer Rind } \\
\text { und / oder Schwein eine andere Tierart enthalten ist, so muss dies in der } \\
\text { Verkehrsbezeichnung kenntlich gemacht werden. }\end{array}$ \\
\hline Kalbswurst & $\begin{array}{l}\text { Leitsätze für Fleisch und Fleischerzeugnisse 2.11: min. 15\% Kalb- } \\
\text { fleisch }\end{array}$ \\
\hline Alkoholfreies Bier & $\begin{array}{l}\text { Wirtschaftsverband Deutscher Brauer-Bund in Anlehnung an die } \\
\text { WeinV: max. 0,5 Vol-\% }\end{array}$
\end{tabular}

\subsection{Produktbeispiel 1: Geflügelwurst}

Durch das Produktbeispiel Geflügelwurst wird das Kennzeichnungsfeld Tierartenkennzeichnung thematisiert. Dabei geht es insbesondere darum, inwieweit die als Zutat verwendete Fleischsorte namensgebend für das Produkt ist und wie bei der Verarbeitung mehrerer Fleischsorten zu verfahren ist. Anwendung findet bei der Bezeichnung Geflügelwurst der generelle Leitsatz für Fleisch und Fleischerzeugnisse Nr. 2.11, da Geflügel als Tierart selbst nicht genannt wird: Steht kein Hinweis einer Tierart in der Verkehrsbezeichnung, so besteht das Produkt aus Rind- und / oder Schweinefleisch. Verwenden Hersteller außer Schweineund Rindfleisch eine weitere oder ausschließlich eine andere Fleischsorte, so ist dies in der Verkehrsbezeichnung durch die Nennung der Tierart kenntlich zu machen. Im Umkehrschluss bedeutet Leitsatz 2.11, dass die Zumischung von Schwein oder Rind zu einer Geflügelwurst nicht in der Verkehrsbezeichnung Ausdruck finden muss.

Die Stiftung Warentest hat im Juni 2011 Geflügelfleischerzeugnisse getestet. Dazu wurden im März 2011207 Geflügelfleischerzeugnisse gekauft, darunter 14 Bio-Produkte. Bei der Auswertung wurden die Etiketten und die Zutatenlisten begutachtet. Bei $51 \%$ der Produkte wird in der Verkehrsbezeichnung eine Geflügelart genannt und das Produkt besteht zu $100 \%$ aus Geflügel im Fleischanteil. $19 \%$ der Produkte tragen Bezeichnungen wie $100 \%$ GeflügelWürstchen. Bei $5 \%$ der Produkte verwenden die Hersteller in der Produktbezeichnung die Formulierung „mit Geflügel“, was dem Verbraucher einen Hinweis gibt, dass das Produkt auch andere Fleischarten wie Schwein oder Rind enthält. $25 \%$ der Produkte tragen eine zusammengesetzte Bezeichnung, in der das Wort „Geflügel-“ vorkommt, wie bspw. „Geflügelwurst", aber keine weitere Tierart in der Verkehrsbezeichnung angesprochen wird. Bei diesen 53 Produkten können Verbraucher erst beim Betrachten der Zutatenliste erkennen, dass die Wurst neben Geflügel- auch Schweine- oder Rindfleisch enthält (Stiftung Warentest 2011). 
Zur Untersuchung des Kennzeichnungsfeldes Tierartenkennzeichnung wurde die Stichprobe in zwei Sub-Samples unterteilt. 512 Teilnehmer wurden zu Geflügelwurst befragt, 509 Probanden bekamen das Produktbeispiel Kalbswurst vorgelegt ${ }^{6}$. Die Fragestellungen waren für die beiden Produkte analog. Die Verbraucher wurden zunächst gefragt, wie sie die Verkehrsbezeichnung Geflügelwurst auffassen. Unter einer Geflügelwurst verstehen demnach 73,6 \% der Verbraucher, dass für eine Wurst mit dieser Bezeichnung ausschließlich Geflügelfleisch verarbeitet wird. 22,7\% sind der Auffassung, dass das Produkt sowohl Schweine- als auch Geflügelfleisch enthält. 3,7\% sind sich nicht sicher, wie die Bezeichnung zu verstehen ist. Die Verteilung ist in Tabelle 2 dargestellt.

Tabelle 2 Verbraucherverständnis „Geflügelwurst“ (Bitte sagen Sie mir, was Sie unter dem Namen , Geflügelwurst" verstehen.)

Wurst, für die ausschließlich Geflügelfleisch verarbeitet worden ist.
Wurst, für die eine Mischung aus Schweine- und Geflügelfleisch verarbeitet worden ist.

\begin{tabular}{l|l|l}
\hline $73,6 \%$ & $22,7 \%$ & $3,7 \%$
\end{tabular}

Die anschließenden Fragen zielten auf die Ermittlung eines Toleranzbereiches namentlich nicht genannter Fleischarten. Zunächst wurden die Teilnehmer darüber informiert, dass Hersteller unterschiedliche Rezepturen verwenden, wobei einige ausschließlich Geflügelfleisch verarbeiten und andere Geflügel- und Schweinefleisch mischen. Dann wurden die Befragten gebeten, auf einem stufenlos verstellbaren Schieberegler einzustellen, welchen Mindestfleischanteil Geflügelfleisch sie in einer Geflügelwurst erwarten. Für Geflügelwurst wird ein durchschnittlicher Geflügelfleischanteil von 85,9\% gefordert. $96 \%$ des Sub-Samples geben einen Anteil von $50 \%$ und mehr an. Bei der Frage, wie viel Schweinefleisch ihrer Meinung nach in einem Produkt mit der Kennzeichnung Geflügelwurst höchstens verarbeitet sein dürfte (Tabelle 3), tolerieren die Probanden im Durchschnitt einen Schweinefleischanteil von 10,4 \%. 41,2 \% der Probanden vertreten die Meinung, dass gar kein Schweinefleisch verarbeitet sein sollte.

\footnotetext{
${ }^{6}$ Es besteht auch innerhalb der beiden Subsamples Repräsentativität.
} 
Tabelle 3 Zusammensetzung „Geflügelwurst“

\begin{tabular}{|c|c|c|}
\hline & Mittelwert & Standardabweichung \\
\hline $\begin{array}{l}\text { Wie viel Prozent Geflügelfleisch sollte Ih- } \\
\text { rer Meinung nach mindestens in einer } \\
\text { Wurst mit dem Namen „Geflügelwurst“" } \\
\text { verarbeitet sein? }\end{array}$ & $85,9 \%$ & 17,328 \\
\hline $\begin{array}{l}\text { Wie viel Prozent Schweinefleisch dürfte } \\
\text { Ihrer Ansicht nach in einer Wurst mit dem } \\
\text { Namen „Geflügelwurst“ höchstens verar- } \\
\text { beitet sein? }\end{array}$ & $10,4 \%$ & 13,224 \\
\hline
\end{tabular}

\subsection{Produktbeispiel 2: Kalbswurst}

Zusätzlich zur Geflügelwurst wurde „Kalb“ als Tierart gewählt, um ggf. Unterschiede zwischen der Kennzeichnung verschiedener Tierarten aufdecken zu können. Um die Produktbeispiele einheitlich und verständlich zu gestalten, wurde der Begriff Kalbswurst gewählt. In der Praxis finden sich neben Kalbswurst verschiedene andere Produktbezeichnungen mit der Tierart „Kalb“.

Nach den Leitsätzen des Deutschen Lebensmittelbuchs für Fleisch und Fleischerzeugnisse (2.11) darf ein Hersteller in der Verkehrsbezeichnung den Begriff „Kalb-“ verwenden, wenn der Fleischanteil des Produktes min. $15 \%$ Kalb- und / oder Jungrindfleisch enthält. Die Begriffe Kalb und Jungrind sind wiederum durch eine Verordnung der EU geregelt (EUKalbfleisch-VermarktungsVO [VO (EG) 566/2008]). Im deutschen Lebensmitteleinzelhandel finden sich verschiedene Produkte, in deren Verkehrsbezeichnung das Wort „Kalb“ auftaucht. Neben der Kalbswurst sind dies Kalbsleberwurst und Kalbfleisch-Leberwurst. Heißt das Produkt Kalbsleberwurst, so muss zudem die Leber vom Kalb stammen. Wählt der Hersteller die Verkehrsbezeichnung Kalbfleisch-Leberwurst, so enthält das Produkt Kalbfleisch, die Leber kann von einer anderen Tierart stammen (Leitsätze für Fleisch und Fleischerzeugnisse, 2.2312.1).

Die praktische Umsetzung durch die Hersteller bei Kalbsleberwurst, Kalbsfleisch-Leberwurst oder Kalbsbratwürsten weicht deutlich voneinander ab. Um die Problematik der unterschiedlichen Zusammensetzungen der Produkte durch die Hersteller einordnen zu können, werden im Folgenden beispielhaft Rezepturen namhafter Hersteller vorgestellt: Lutz Kalbsleberwurst der Lutz Fleischwaren GmbH besteht zu $15 \%$ aus Kalbfleisch und zu $5 \%$ aus Kalbsleber. Einer Kalbsleberwurst der Westfälischen Fleischwarenfabrik Stockmeyer GmbH wird zu $15 \%$ Kalbfleisch beigemengt. „Du darfst Feine Kalbfleisch Leberwurst“ der Unilever 
Deutschland $\mathrm{GmbH}$ enthält $17 \%$ Kalbfleisch. Der Bio-Hersteller Fleischwerke E. Zimmermann GmbH \& Co. KG hat eine Kalbsfleisch-Leberwurst im Sortiment, die den gesetzlichen Mindestanteil von $15 \%$ Kalbfleisch enthält. Bei Kalbsbratwürsten fallen die Kalbfleischanteile höher aus. Die Kalbsbratwurst der Kurstadtmetzgerei Fries hat einen Kalbfleischanteil von $60 \%$. Es gibt aber auch Beispiele von Herstellern, die für ihre Produkte als Fleischsorte ausschließlich Kalbfleisch verarbeiten. Die Kalbsbratwurst des FleischereiFachgeschäftes Wiedemann enthält zu 100 \% Kalbfleisch im Fleischanteil.

Die Befragung der Probanden zur Kalbswurst erfolgte analog zu Geflügelwurst. Tabelle 4 gibt die prozentuale Verteilung der Antwortmöglichkeiten wieder.

Tabelle 4 Verbraucherverständnis „Kalbswurst“ (Bitte sagen Sie mir, was Sie unter dem Namen „Kalbswurst" verstehen.)

Wurst, für die ausschließlich Kalbfleisch verarbeitet worden ist.
Wurst, für die eine Mischung aus Schweine- und Kalbfleisch verarbeitet worden ist.

$66,4 \%$

$30,3 \%$

$3,3 \%$

$66,4 \%$ der Teilnehmer erwarten von einer Kalbswurst, dass ausschließlich Kalbfleisch im Produkt verarbeitet wird. 30,3\% sind der Auffassung, dass eine Mischung aus Schweine- und Kalbfleisch vorhanden ist. 3,3\% geben an, dass sie sich nicht sicher sind, wie die Produktbezeichnung zu verstehen ist.

Nachdem die Probanden Informationen über die unterschiedliche Kennzeichnungspraxis der Hersteller erhalten haben, wird bei Kalbswurst im Mittel 82,6 \% Kalbfleischanteil im Produkt vorausgesetzt (Tabelle 5). 93 \% erwarten, dass mindestens die Hälfte des Fleisches vom Kalb stammt. Zuletzt wurden die Teilnehmer gebeten anzugeben, wie viel Schweinefleisch in einer Kalbswurst höchstens verarbeitet werden dürfe. Auch hier wurde wieder ein stufenlos verstellbarer Schieberegler vorgelegt. Im Ergebnis tolerieren die Verbraucher im Durchschnitt einen verarbeiteten Schweinefleischanteil von 13,5\%. $33 \%$ halten Schweinefleisch in einem Produkt mit der Bezeichnung Kalbswurst für inakzeptabel. 
Tabelle 5 Zusammensetzung „Kalbswurst“

\begin{tabular}{l|ll} 
& Mittelwert & Standardabweichung \\
\hline $\begin{array}{l}\text { Wie viel Prozent Kalbsfleisch sollte Ihrer } \\
\text { Meinung nach mindestens in einer Wurst mit } \\
\text { dem Namen „Kalbswurst“ verarbeitet sein? }\end{array}$ & $\mathbf{8 2 , 6} \%$ & $\mathbf{1 8 , 7 1 5}$ \\
\hline $\begin{array}{l}\text { Wie viel Prozent Schweinefleisch dürfte Ihrer } \\
\text { Ansicht nach in einer Wurst mit dem Namen } \\
\text { „Kalbswurst“ höchstens verarbeitet sein? }\end{array}$ & $\mathbf{1 5 , 5 \%}$ & $\mathbf{1 5 , 0 2 1}$
\end{tabular}

\subsection{Produktbeispiel 3: Alkoholfreies Bier}

Die Verwendung der Kennzeichnung ,alkoholfreies Bier“ ist in Deutschland gesetzlich nicht speziell geregelt. Allgemein muss der Alkoholgehalt in einem Getränk in Deutschland erst angegeben werden, wenn dieser über 1,2 Vol.-\% liegt ( 3 Abs. 1 S. 5 LMKV). Für die Feststellung der Verkehrsbezeichnung greift in diesem Fallbeispiel die verkehrsübliche Bezeichnung, wie sie der zuständige Wirtschaftsverband vertritt: Der Deutsche Brauer-Bund orientiert sich an der WeinV (Deutscher Brauer-Bund 2013). Demnach darf ein Wein als alkoholfrei deklariert werden, wenn er max. 0,5 Vol.-\% enthält ( $\$ 47$ Abs. 1 S. 2 WeinV). Zudem verweist der Deutsche Brauer-Bund darauf, dass die Bezeichnung alkoholfreies Bier in anderen europäischen Ländern erlaubt ist. In $\S 47$ der WeinV wird allerdings auch der Begriff ,alkoholreduziert" verwendet.

In der Praxis werden Biere mit z. T. sehr unterschiedlichen Alkoholgehalten als alkoholfrei deklariert und vermarktet. Die Spanne umfasst sowohl Produkte wie die Marke Warsteiner Premium alkoholfrei der Warsteiner Brauerei, das gar keinen Alkohol enthält (0,0 Vol.-\%), als auch Biere mit höheren Alkoholgehalten wie z. B. das Clausthaler Classic Premium alkoholfrei der Radeberger Gruppe mit einem Alkoholgehalt von 0,45 Vol.-\%. Laut Clausthaler bedarf es einer gewissen Menge Alkohols, um den typischen Geschmack zu gewährleisten. Im Ausland gibt es z. T. strengere Regeln. So darf in Großbritannien ein Produkt nur bis max. 0,05 Vol.-\% als alkoholfrei deklariert werden, weshalb dasselbe Bier der Radeberger Gruppe in Großbritannien mit „low alcohol“ gekennzeichnet wird (Clausthaler 2013).

Um Erkenntnis darüber zu erlangen, wie Verbraucher die Kennzeichnung alkoholfreies Bier verstehen, sollten die Probanden in der Befragung angeben, ob sie Alkohol in einem so gekennzeichneten Bier erwarten. Auf einer fünfstufigen Likert Skala von „Ja, auf jeden Fall“ bis „Nein, auf keinen Fall“ wurde das Verbraucherverständnis mit zwei Items abgefragt. Dabei wurden die Antwortmöglichkeiten randomisiert vorgelegt. 69,2\% der Teilnehmer verstehen 
unter der Kennzeichnung alkoholfreies Bier, dass das Getränk keinen Alkohol enthält. Nur 30,3 \% vermuten, dass das Bier eine gewisse Menge an Alkohol enthält. Die genaue Verteilung kann Tabelle 6 entnommen werden.

Tabelle 6 Verbraucherverständnis ,alkoholfreies Bier“6

\begin{tabular}{|c|c|c|c|c|c|c|c|}
\hline $\begin{array}{l}\text { Ich verstehe unter } \\
\text { der Bezeichnung } \\
\text { „alkoholfreies Bier“, } \\
\text { dass das Bier... }\end{array}$ & $\begin{array}{l}\text { Ja, auf } \\
\text { jeden } \\
\text { Fall. }\end{array}$ & $\mathrm{Ja}$ & $\begin{array}{l}\text { Bin mir } \\
\text { unsicher }\end{array}$ & Nein & $\begin{array}{l}\text { Nein, } \\
\text { auf kei- } \\
\text { nen } \\
\text { Fall. }\end{array}$ & $\begin{array}{l}\text { Mittel- } \\
\text { wert }\end{array}$ & $\begin{array}{l}\text { Stand- } \\
\text { ardab- } \\
\text { weich- } \\
\text { ung }\end{array}$ \\
\hline $\begin{array}{l}\text {...keinen Alkohol } \\
\text { enthält. }\end{array}$ & $42,9 \%$ & $26,3 \%$ & $10,6 \%$ & $16,2 \%$ & $4,0 \%$ & $\mathbf{0 , 8 8}$ & 1,237 \\
\hline $\begin{array}{l}\text {...geringe Mengen } \\
\text { Alkohol enthält (bis } \\
0,5 \text { Vol.-\%). }\end{array}$ & $8,9 \%$ & $21,4 \%$ & $16,7 \%$ & $31,6 \%$ & $21,4 \%$ & $-\mathbf{0 , 3 5}$ & 1,237 \\
\hline
\end{tabular}

Für die Tierartenkennzeichnung sind keinerlei signifikante Zusammenhänge zur Soziodemografie nachzuweisen, was an Hand von ANOVAs, Korrelationen und Kreuztabellen überprüft wurde. Für das Fallbeispiel alkoholfreies Bier liegt lediglich ein signifikanter Zusammenhang zum Geschlecht vor. Signifikant (0,1 \% Niveau) weniger Männer glauben, dass ein alkoholfreies Bier keinen Alkohol enthält und signifikant (0,1 \% Niveau) mehr Männer versteht die Bezeichnung so, dass geringe Mengen Alkohol enthalten sind.

\section{Diskussion der Fallbeispiele}

Insgesamt lässt sich festhalten, dass in den drei untersuchten Produktbereichen unterschiedliche Produktqualitäten unter derselben Verkehrsbezeichnung vermarktet werden. Das Verbraucherverständnis weicht in allen drei Beispielen von der tatsächlichen Beschaffenheit eines Teils der Produkte ab. Es ist daher wenig verwunderlich, dass diese Fallkomplexe auch in den Medien kontrovers diskutiert werden.

Für die Produktbeispiele Geflügelwurst und Kalbswurst kann festgestellt werden, dass der Großteil der Verbraucher an Hand der Verkehrsbezeichnung schlussfolgert, von welchen Tierarten Fleisch in einem Fleischprodukt verarbeitet worden ist. Aus Verbrauchersicht ist diese Information nicht unwesentlich. Neben religiösen Gründen (vgl. Roth 2008) können Verbraucher auch aus gesundheitlichen Gründen bestimmte Fleischarten bevorzugen, bspw. auf Grund eines niedrigen Fettgehaltes (Hauner 2013).

Aus Herstellersicht gibt es mehrere Gründe, Fleisch verschiedener Tierarten in einem Produkt zu verarbeiten. Neben Kostenüberlegungen spielen auch technologische und sensorische Ar- 
gumente eine Rolle. Schweinefleisch ist für den Produzenten günstiger im Einkauf als Kalbfleisch, sodass eine Kalbswurst, der Schweinefleisch beigemengt ist, zu niedrigeren Kosten herzustellen ist. Bei Geflügelwurst spielt dieses Argument keine Rolle, da Geflügelfleisch preiswerter als Schweinefleisch ist. Vielmehr wurden hier in der Vergangenheit sensorische und produktionstechnische Argumente angeführt. Aus technologischen Gründen ist es allerdings heute nicht mehr notwendig, Schweinefleisch bei der Geflügelwurstproduktion einzusetzen. Wie die Bespiele in Kapitel 5.1 verdeutlichen, gelingt es vielen Produzenten, Produkte zu $100 \%$ aus Geflügelfleisch herzustellen. Anstelle von Speck kann z. B. Geflügelfett oder Pflanzenfett eingesetzt werden (Stiftung Warentest 2011). So ist auch die Sensorik nicht zwangsläufig schlechter zu bewerten als bei Produkten mit der Verwendung von Schweinespeck. Die technologischen Entwicklungen lassen es damit zu, ein den überwiegenden Verbrauchererwartungen entsprechendes Wurstprodukt herzustellen.

Eine vergleichbare Problematik zeigt sich für das dritte Produktbeispiel. Eine klare Mehrheit der Verbraucher versteht die Kennzeichnung von ,alkoholfreiem Bier““ so, dass es gar keinen Alkohol enthält. Für verschiedene Verbrauchergruppen, wie abstinente Alkoholiker oder Konsumenten, die aus religiösen Gründen auf Alkohol verzichten, ist eine korrekte und eindeutige Deklaration wünschenswert. Während vormals aus technologischen und sensorischen Gründen ein geringer Alkoholgehalt im Bier verblieb, gibt es schon seit einiger Zeit Biere auf dem Markt, die einen Alkoholgehalt von 0,0 Vol.-\% haben. Das spricht dafür, den Unterschied der Herstellungsweise und damit auch den Innovationsgehalt des Herstellers durch eine entsprechende Kennzeichnung auszuweisen. Dies fördert ähnlich wie bei der Wurstkennzeichnung den Wettbewerb und dient der Marktdifferenzierung. Verbraucher können dann informierter entscheiden, welches Produkt sie bevorzugen.

In beiden Fällen (Geflügelwurst und alkoholfreies Bier) wäre es heute umsetzbar, Verkehrsbezeichnungen so eng zu fassen, dass diese stärker selbsterklärend sind und kaum noch Potenzial für Fehlinterpretationen bieten. Damit geht es um die Frage der Anpassung tradierter Regeln für Verkehrsbezeichnungen an geänderte technologische Bedingungen vor dem Hintergrund von Verbrauchererwartungen. Offensichtlich hat z. B. die in der Vergangenheit gängige und von der Lebensmittelbuch-Kommission zugelassene Praxis des Fleisch-Mix nicht dazu geführt, dass sich das Verbraucherverständnis an die Regelungen der Herstellungspraxis angepasst hat. Es muss offen bleiben, ob dies das Ergebnis geringer Informationsbemühungen der Konsumenten oder wenig aufklärender Kommunikationsmaßnahmen der Hersteller ist (vgl. Kühl et al. 2013). Jedenfalls führt die derzeitige Regelungspraxis wie aufgezeigt zu ei- 
nem hohen Irreführungspotenzial und zahlreichen negativen Medienberichten (Brühl 2011; Kirschey und Hauer 2012; Kuhlmann 2012).

\section{Schlussfolgerungen}

Verbraucherpolitik soll den Konsumenten vor Irreführung und Täuschung schützen (BMELV o. J.; vgl. Lebensmittel-Informationsverordnung VO 1169/2011/EG). Auch wenn ein Produkt im Zutatenverzeichnis viele detaillierte Informationen liefert, so haben bzw. nehmen sich viele Verbraucher realiter kaum die Zeit, diese bei jedem Produkt zu lesen, zu verstehen und miteinander zu vergleichen. Die in 2014 in Kraft tretende Lebensmittelinformationsverordnung (LMIV) der Europäischen Union weist explizit darauf hin, dass Lebensmittel klar und verständlich gekennzeichnet sein müssen (VO 1169/2011/EG, Erwägungsgrund 9; Art. 7 Abs. 2 VO 1169/2011/EG).

Um dem Verbraucher zu einer rationaleren Entscheidung zu verhelfen, ist es vor dem Hintergrund des faktischen Verbraucherverhaltens wichtig, auf der Produktvorderseite prägnante, entscheidende Informationen zu liefern (Dantas 2004; van Herpen und van Trijp 2011; Zühlsdorf et al. 2013). Eine solche Schlüsselinformation kann die Verkehrsbezeichnung sein. Aus den vorliegenden Ergebnissen der Studie lässt sich schlussfolgern, dass die deutschen Verbraucher die Verkehrsbezeichnungen in den Fallbeispielen nicht ohne weitere Erklärung so verstehen, wie es der Produktzusammensetzung entspricht.

Die fehlende Anpassung der Definition der Verkehrsbezeichnungen an die Verbrauchererwartungen führt in den drei untersuchten Fällen zu Fehleinschätzungen bei Verbrauchern und zu einem hohen Maß an wahrgenommener Täuschung. Die Lebensmittelkennzeichnung muss die Interessen von Verbrauchern, Produzenten und Herstellern gleichermaßen berücksichtigen. Im Zweifelsfall wiegen jedoch die Verbraucherinteressen auf Grund des Schutzes der Verbraucher vor Irreführung und Täuschung stärker als die der Wirtschaft (Hohmann 1994, 382). Die Hersteller sollten dabei ebenfalls ein Interesse daran haben, das Vertrauen ihrer Kunden zu erlangen und Transparenz zu schaffen. In diesem Sinne ist es zu begrüßen, wenn der Deutsche Brauer-Bund sich nicht zuletzt vor dem Hintergrund der hier vorgestellten Ergebnisse bereit erklärt hat, den Restalkoholgehalt ab 2015 zu kennzeichnen. Im Sinne der Verständlichkeit der herausgehobenen Verkehrsbezeichnung vermag allerdings die britische Unterscheidung in alkoholfrei und alkoholarm noch mehr zu überzeugen. 


\section{Limitationen}

Die Studie beruht auf drei Fallbeispielen und lässt daher keine Aussage über die Breite möglicher Probleme bei der Definition von Verkehrsbezeichnungen zu. Sie dient zunächst einmal zur Darlegung des grundsätzlichen Problems und beleuchtet spezifisch den dynamischen Charakter der Fragestellung mit dem Verweis auf neue Technologien, die eine Änderung von Verkehrsbezeichnungen im Zeitablauf sinnvoll erscheinen lassen. Hier wäre z. B. mit Blick auf die Regelungspraxis in der Lebensmittelbuchkommission genauer zu untersuchen, warum solche Revisionen zumindest in den vorliegenden Fällen nicht erfolgt sind. Möglicherweise könnte es sinnvoll sein, in der Lebensmittelbuchkommission, aber auch in der Rechtsprechung oder von Seiten des Gesetzgebers, häufiger demoskopische Studien einzusetzen, um Hinweise auf ein abweichendes Verbraucherverständnis zu erhalten. Der Verweis auf die hohen Kosten einer Befragung ist nachvollziehbar, verblasst aber vor dem Hintergrund der beachtlichen Verunsicherung der Verbraucher.

Interessant wäre es auch, in weiteren Studien zu untersuchen, ob der Verbraucher beim Einkaufen gezielt die Verkehrsbezeichnung eines Produktes sucht. Es ist in der LMKV nicht geregelt, wo diese sich auf der Fertigverpackung zu befinden hat. Oft ist die Verkehrsbezeichnung nur auf der Verpackungsrückseite zu finden. Es sollte untersucht werden, wie stark der Verbraucher Informationen an welcher Stelle der Verpackung in seinen Entscheidungsprozess einbezieht.

\section{Literatur}

Akerlof GA (1970) The Market for "Lemons": Qualitative Uncertainty and the Market Mechanism. Quarterly Journal of Economics. 84(3):488-500

BMELV Bundesministerium für Ernährung, Landwirtschaft und Verbraucherschutz (2013a) Hinweise für die Anwendung der Leitsätze des deutschen Lebensmittelbuchs. http://www.bmelv.de/SharedDocs/Standardartikel/Ernaehrung/SichereLebensmittel/Kennz eichnung/Lebensmittelbuch/HinweiseLeitsaetzeLebensmittelbuch.html. Abgerufen am 22. April 2013

BMELV Bundesministerium für Ernährung, Landwirtschaft und Verbraucherschutz (2013b) Das Deutsche Lebensmittelbuch. http://www.bmelv.de/SharedDocs/Standardartikel/ Ernaehrung/SichereLebensmittel/Kennzeichnung/Lebensmittelbuch/Deutsches Lebensmittelbuch.html. Abgerufen am 8. Februar 2013

BMELV Bundesministerium für Ernährung, Landwirtschaft und Verbraucherschutz (2013c) BMELV-Initiative „Klarheit und Wahrheit“. $\quad$ http://www.bmelv.de/SharedDocs/Stand ardartikel/Ernaehrung/SichereLebensmittel/Kennzeichnung/KlarheitUndWahrheit/Klarheit UndWahrheitBeiLebensmitteln.html. Abgerufen am 7. August 2013 
BMELV Bundesministerium für Ernährung, Landwirtschaft und Verbraucherschutz (o. J.) Verbraucherschutz und Informationsrechte. http://www.bmelv.de/DE/EuropaInternationales/Verbraucherpolitik/verbraucherpolitik_node.html. Abgerufen am 26. September 2013

Brühl J (2011) Irreführende Lebensmittelpackungen - Erdbeeren, mit Datteln gestreckt. Süddeutsche.de. Wirtschaft. http://www.sueddeutsche.de/wirtschaft/irrefuehrendelebensmittelpackungen-erdbeeren-mit-datteln-gestreckt-1.1129249. Abgerufen am 15. Oktober 2013

Clausthaler (2013) Info Center FAQ. http://us.clausthaler.com/cms/startordner/infocenter/ 1390_u1.html. Abgerufen am 26. Februar 2013

Dantas MIS, Minim VPR, Rosires D, Puschmann R (2004) The effect of packaging on the perception of minimally processed products. Journal of International Food \& Agribusiness Marketing. 16 (2): 71-83

Deutsch V (1996) Der Einfluss des europäischen Rechts auf den Irreführungstatbestand des § 3 UWG - Gedanken zum Verbraucher-Leitbild und zur Relevanz bei Täuschungen. Gewerblicher Rechtsschutz und Urheberrecht. 541, 545

Deutscher Brauer-Bund e.V. (2013) Alkoholfreies Bier. http://www.brauerbund.de/aktuell/alkoholfreies-bier.html. Abgerufen am 25. Februar 2013

Grube C (1997) Verbraucherschutz durch Lebensmittelkennzeichnung? Eine Analyse des deutschen und europäischen Lebensmittelkennzeichnungsrechts. Springer Verlag, Berlin u. a.

Harper L, Souta P, Ince, J., Mckenzie J (2007) Food Labelling Consumer Research: What Consumers Want. A Literature Review. http://www.ncbi.nlm.nih.gov/pubmed/21383370. Abgerufen am 09. Februar 2013

Hauner H (2013) Ernährungstherapie des Typ-2-Diabetes. Der Diabetologe 9 (5): $405-416$

Hecker B (2001) Strafbare Produktwerbung im Lichte des Gemeinschaftsrechts. Europäisierung des deutschen Täuschungsschutzstrafrechtes am Beispiel des Lebensmittel-, Wettbewerbs- und Betrugsstrafrechts. Mohr-Siebeck, Tübingen

Helm H (2010) Irreführende geschäftliche Handlungen und Unterlassungen. In: Gloy/Loschelder/Erdmann, § 59. Handbuch des Wettbewerbsrechts. 4. Auflage. Verlag C. H. Beck, München

Hohmann G (1994) Die Verkehrsauffassung im deutschen und europäischen Lebensmittelrecht. P.O.C.-Verlag, Bayreuth

Iyengar R, Anasar A, Gupta S (2003) Leveraging Information Across Categories. Quantitative Marketing and Economics. 1(4): 425-465

Iyengar SS, Lepper MR (2000) When Choice is Demotivating: Can One Desire too much of a Good Thing? Journal of Personality and Social Psychology. 79(6): 995-1006

Kirschey R, Hauer T (2012) Traumfabrik Essen - Das Märchen vom natürlichen Lebensmittel. 3 sat. Scobel. http://www.3sat.de/page/?source=/scobel/160819/index.html. Abgerufen am 15. Oktober 2013

Köhler H, Bornkamm J (2013) Gesetz gegen den unlauteren Wettbewerb. Preisangabenverordnung, Unterlassungklagegesetz, Dienstleistungs-InformationspflichtenVerordnung. 31. Auflage. Verlag C. H. Beck, München 
Kolodinsky J (2012) Persistence of Health Labeling Information Asymmetry in the United States: Historical Perspectives and Twenty-First Century Realities. Journal of Macromarketing. 32(2): 193-207

Kroeber-Riel W, Esch F-R (2004) Strategie und Technik der Werbung: Verhaltenswissenschaftliche Ansätze. Kohlhammer GmbH, Stuttgart

Kühl R, Mäncher A, Piper A (2013) Lebensmittelklarheit.de und Verbraucherpolitik - oder wie viel Schutz verdient Ignoranz?. Diskussionspapier Nr. 2013-1. Universität Gießen

Kuhlmann S (2012) Irreführende Bezeichnungen von Lebensmitteln. Deutschlandfunk. Umwelt und Verbraucher. http://www.dradio.de/dlf/sendungen/umwelt/1960820/. Abgerufen am 15. Oktober 2013

Orth UR, Malkewitz K (2008) Holistic Package and Consumer Brand Impressions. Journal of Marketing. 36(5): 706-712

Pflüger A (2010) Ermittlung der Verkehrsauffassung durch demoskopische Umfragen. In: Gloy/Loschelder/Erdmann, § 42. Handbuch des Wettbewerbsrechts. 4. Auflage. Verlag C. H. Beck, München

Roth K (2008) Chemische Produktion gemäß Koran und Thora. Chemie in unserer Zeit 42 (1):42-51

Schrankel H (2005) Zeitverwendung der privaten Haushalte Vergleich der Zeitbudgeterhebungen 2001/02 gegenüber 1991/92. In: Seifert/Möhring/Zühlke/Schrankel: Statistische Analysen und Studien. 24. http://www.it.nrw.de/statistik/analysen/stat_studien/2005/band_24/schrankel_24_2005.pdf. Abgerufen am 12. März 2013

Sørensen HS, Clement J, Gabrielsen, G (2012) Food Labels - an Exploratory Study into Lable Information and what Consumers See and Understand. The International Review of Retail, Distribution and Consumer Reseach. 22(1): 101-114

Sosnitza O (2010) Gesetz gegen den unlauteren Wettbewerb: mit Preisangabenverordnung; Kommentar. In: Ohly/Sosnitza. Gesetz gegen den unlauteren Wettbewerb: mit Preisangabenverordnung; Kommentar. 5. Auflage 2010. Beck, München

Statistisches Bundesamt (2012) Statistisches Jahrbuch. Deutschland und Internationales. Wiesbaden.

https://www.destatis.de/DE/Publikationen/StatistischesJahrbuch/StatistischesJahrbuch2012 .pdf?_blob=publicationFile. Abgerufen am 29. Juli 2013

Stiftung Warentest (2011) Geflügelwurst. http://www.test.de/filestore/4272420_t201109020. pdf?path=/protected/24/71/7afdae8f-e1d4-4a9f-b692-dc2638f068ad-protectedfile.pdf\&key =D674D4FF9BAC5175854255D637630E17C3A94531. Abgerufen am 1. März 2013

Tilmann W (1984) Die Verkehrsauffassung im Wettbewerbs- und Warenzeichenrecht Möglichkeiten und Grenzen der demoskopischen Wahrheitsfindung im Prozess. GRUR 1984. $716 f$

Van Herpen E, Van Trijp HCM (2011) Front-of-pack nutrition labels. Their effect on attention and choices when consumers have varying goals and time constraints. Appetite. 57(1):148-160

Van Kleef E, Van Trijp H, Paeps F, Fernández-Celemín L (2008) Consumer preferences for front-of-pack calories labelling. Public health nutrition 11(2):203-13 
Zühlsdorf A, Nitzko S, Spiller A (2013) Kennzeichnung und Aufmachung von Lebensmitteln aus Sicht der Verbraucher: Empirische Untersuchungsbefunde. Begleitende Verbraucherforschung zum Internetportal www.lebensmittelklarheit.de der Verbraucherzentralen und des Verbraucherzentrale Bundesverbands, Göttingen 
Anhang (electronic supplement)

Supplementary Tabelle 1 Soziodemografie der Stichprobe

\begin{tabular}{|c|c|c|}
\hline Merkmal & $\begin{array}{l}\text { Häufigkeit }(\%) \text { in der } \\
\text { Stichprobe }\end{array}$ & Häufigkeit (\%) BRD \\
\hline \multicolumn{3}{|l|}{ Altersgruppen } \\
\hline 16 bis 30 & 25,0 & 24,8 \\
\hline 31 bis 50 & 41,2 & 41,2 \\
\hline Über 50 & 33,8 & 34 \\
\hline Altersdurchschnitt & 43,0 & - \\
\hline \multicolumn{3}{|l|}{ Geschlecht } \\
\hline Männer & 50,4 & 50,4 \\
\hline Frauen & 49,6 & 49,6 \\
\hline \multicolumn{3}{|l|}{ Einkommensklassen } \\
\hline Unter 500 Euro & 1,0 & \multirow{2}{*}{5,7} \\
\hline $500-999$ Euro & 5,1 & \\
\hline $1000-1499$ Euro & 9,2 & 9,5 \\
\hline $1500-1999$ Euro & 13,6 & 13,8 \\
\hline $2000-2499$ Euro & 14,6 & 15 \\
\hline $2500-2999$ Euro & 13,4 & 12,6 \\
\hline 3000 Euro und mehr & 42 & 43 \\
\hline Keine Angabe & 1,1 & 0,4 \\
\hline \multicolumn{3}{|l|}{ Region } \\
\hline Nord & 15,6 & 15,9 \\
\hline West & 35,5 & 35,6 \\
\hline Süd & 27,9 & 28 \\
\hline Ost & 21,1 & 20,5 \\
\hline \multicolumn{3}{|l|}{ Schulabschluss } \\
\hline $\begin{array}{l}\text { Volksschul- / } \\
\text { Hauptschulabschluss }\end{array}$ & 36,3 & 36,6 \\
\hline Realschulabschluss / POS & 28,8 & 28,8 \\
\hline $\begin{array}{l}\text { (Fach-) Hochschulreife / } \\
\text { (Fach-) Abitur }\end{array}$ & 13,6 & 13,6 \\
\hline (Fach-) Hochschulabschluss & 13,5 & 13,2 \\
\hline $\begin{array}{l}\text { Kein Abschluss / noch in der } \\
\text { Schulausbildung }\end{array}$ & 7,7 & 7,8 \\
\hline
\end{tabular}




\begin{tabular}{|l|l|l|}
\hline Haushaltsgröße & & \\
\hline 1 Person & 40,3 & 40,4 \\
\hline 2 Personen & 33,8 & 34,2 \\
\hline 3 Personen & 12,8 & 12,6 \\
\hline 4 Personen und mehr & 13,1 & 12,8 \\
\hline Lebenssituation & & \\
\hline Bei meinen Eltern & 2,4 & - \\
\hline In einer Wohngemeinschaft & 1,2 & - \\
\hline $\begin{array}{l}\text { Mit meinem Partner (Kinder } \\
\text { ausgezogen) }\end{array}$ & 11,7 & - \\
\hline $\begin{array}{l}\text { Mit meinem Partner (ohne } \\
\text { Kinder) }\end{array}$ & 18,6 & - \\
\hline Mit Partner und Kindern & 22,0 & - \\
\hline Allein erziehend mit Kindern & 3,7 & - \\
\hline Bei meinen Kindern & 0,1 & - \\
\hline Allein & 40,3 & 40,4 \\
\hline
\end{tabular}

Quelle: eigene Berechnungen; Statistisches Bundesamt (2012) 


\section{Teil II: Consumer understanding of food packaging}

II.2 Verbraucherverständnis der Gestaltung von Lebensmittelverpackungen

Autoren: Ramona Weinrich, Sina Nitzko, Achim Spiller, Anke Zühlsdorf

Veröffentlicht in Schriften der Gesellschaft für Wirtschafts- und Sozialwissenschaften des Landbaus e.V. „Neuere Theorien und Methoden in den Wirtschafts- und Sozialwissenschaften des Landbaus “, 2015: Band 50, im Druck 


\section{Zusammenfassung}

Viele Verbraucher interessieren sich für die Qualität von Lebensmitteln und deren Eigenschaften und Zusammensetzung. Wichtige Schlüsselinformationen über die Beschaffenheit eines Produktes können Verbraucher auf der Verpackung von Produkten finden. Solch eine Information kann die Verkehrsbezeichnung darstellen, die eine kurze Charakterisierung der Produktart darstellt und auf die eindeutige Benennung eines Produktes für den Verbraucher abzielt, sodass dieser das Erzeugnis von anderen unterscheiden kann. Weiterhin liefern der Produktname und Produkt- bzw. Zutatenabbildungen auf der Verpackungsvorderseite Informationen für Verbraucher. Auch Clean Label können prägnante Schlüsselinformationen über ein Lebensmittel vermitteln. Mittels einer Verbraucherbefragung (näherungsweise repräsentativ für Alter, Geschlecht, Einkommen, regionale Verteilung, Bildung und Haushaltsgröße in Deutschland, $\mathrm{n}=1.021$ ) wird analysiert, inwieweit die Verpackungsaufmachung bei Verbrauchern Fehlassoziationen über die tatsächliche Produktbeschaffenheit auslöst. Untersucht wird das Verbraucherverständnis in den drei Fallgruppen Verkehrsbezeichnung, Produktbzw. Zutatenabbildung und Clean Label. Anhand der Ergebnisse der Studie lässt sich aufzeigen, dass Verbraucher die Lebensmittelkennzeichnungen vielfach anders verstehen, als es der tatsächlichen Beschaffenheit des Produktes entsprechen würde.

\section{Keywords}

Lebensmittelkennzeichnung, Verbrauchertäuschung, Irreführung, Verkehrsauffassung, Front of Pack Labelling

\section{$1 \quad$ Einleitung}

Heutzutage sieht sich der Verbraucher einer großen Auswahl an Produkten im Lebensmitteleinzelhandel gegenüber. Dabei nimmt die Zeit, die er zum Einkaufen verwendet, tendenziell ab (SchRANKEL 2005). Das Resultat ist eine Verschärfung der von der Marketingforschung schon seit geraumer Zeit konstatierten Informationsüberlastung (KOLODINSKY 2012; KROEBER-RIEL und EsCH 2004; VAN KLEEF et al. 2008). Verbraucher nehmen Informationen nur in limitierter Form auf (SøRENSEN et al. 2012), visuelle Informationen dominieren.

Umso wichtiger ist es für Verbraucher, dass sie sich auf Schlüsselelemente einer Lebensmittelverpackung verlassen können. Verbraucher ziehen Schlüsselelemente heran, um die Beschaffenheit und damit auch die Zusammensetzung eines Produktes beurteilen zu können. 
Solch ein Element ist z. B. die Verkehrsbezeichnung, die es Verbrauchern ermöglichen soll, den Inhalt eines Produktes auf den ersten Blick zu identifizieren ( $\$ 4$ Abs. 1 S. 2 LMKV). Weitere Schlüsselelemente stellen Produktname und Produkt- bzw. Zutatenabbildung dar, die Rückschlüsse auf einzelne Bestandteile zulassen. Ein drittes Kennzeichnungsfeld, das in den letzten Jahren bei Verpackungsaufmachungen deutlich zugenommen hat, sind Clean Label (,saubere Etiketten“). Sie sollen ggü. Konkurrenzprodukten herausstellen, dass auf kritische Inhaltsstoffe mit Negativimage in der Rezeptur verzichtet wird. Solche Signalling Cues sind ein zentrales Element des Qualitätswettbewerbs (FERNQUIST und EKELUND 2014; KIRMANI und RAO 2000; VerbeKE 2013). Für einen funktionierenden Markt ist es wichtig, dass ausgelobte Produkteigenschaften so kommuniziert werden, dass Verbraucher sie richtig verstehen. Nur so kann gewährleistet werden, dass keine Verdrängung lauterer Wettbewerber aus dem Markt stattfindet (AKERLOF 1970) und dass Verbraucher durch Schlüsselelemente die Produktbeschaffenheit erkennen können. Bisher wurden diese drei genannten Kennzeichnungsfelder in der Marketingforschung kaum beachtet; bisherige Veröffentlichungen beschäftigen sich schwerpunktmäßig mit Food Labelling - jedoch nicht auf die zunehmende Praxis des Clean Labellings - (z. B. HARPER et al. 2007), Health Claims (z. B. DE LA HunTY et al. 2014) und der Nährwertkennzeichnung auf Lebensmittelverpackungen (z. B. BIALKOVA und VAN TRIJP 2010).

In dem vorliegenden Artikel soll diskutiert werden, inwiefern ausgewählte Kennzeichnungselemente auf Lebensmittelverpackungen Falschassoziationen über die Produktbeschaffenheit beim Verbraucher auslösen können. Hierzu wird die Verbraucherauffassung von Signalling Cues in Bezug auf drei Fallgruppen analysiert: (1) Verkehrsbezeichnung, (2) Produktname in Verbindung mit Produkt- und Zutatenabbildungen in Bezug auf die Zutatenliste und (3) Clean Label. Die Produktbeispiele der Fallgruppen sollen aufzeigen, ob die derzeitige Kennzeichnungspraxis bei Lebensmittelverpackungen dem Verbraucherverständnis gerecht wird.

\section{Aufmachung von Lebensmittelverpackungen: Stand der Forschung zur Ver- braucherwahrnehmung}

Die Lebensmittelverpackung gibt Verbrauchern beim Lebensmitteleinkauf Informationen über die Beschaffenheit eines Produktes, die in die Kaufentscheidung mit einfließen (FRENQUIST und EKELUND 2014). Solche Informationen werden u. a. durch die Verkehrsbezeichnung, den Produktnamen, verbale Claims, Label und Produkt- oder Zutatenabbildungen vermittelt. 
Bisher finden sich in der Literatur in großer Mehrzahl Studien zur Ernährungskennzeichnung auf der Verpackungsvorderseite und den verschiedenen Wirkungen dieser (COWBURN und StOCKLEY 2004; FeunEKES et al. 2008; Grunert et al. 2010). BialKOVA und VAN TriJP (2010) bestimmen weiterhin in einer Studie die Faktoren, die die Aufmerksamkeit auf die Nährwertkennzeichnung ziehen. Diese sind Größe, Farbe, Vertrautheit mit der Nährwertkennzeichnung und die Platzierung auf die Verpackungsvorderseite. Die Ergebnisse lassen den Schluss zu, dass visuelle Reize wie Produkt- oder Zutatenabbildungen wichtige Schlüsselinformationen für Verbraucher darstellen. Eine Studie von KÖNIGSTORFER und GRÖPPELKLEIN (2012) deckt auf, dass Verbraucher die Informationen auf der Verpackungsvorderseite nicht länger als 29 bis 351 msec. fixieren, was jedoch nach RAYNER (1998) nicht ausreicht, um die Verarbeitung von numerischen Informationen zu gewährleisten. Daraus resultiert, dass Schlüsselinformationen auf Verpackungen für Verbraucher einen hohen Stellenwert haben, um die Beschaffenheit eines Produktes in kurzer Zeit erkennen zu können. Dass Lebensmittelverpackungen für Verbraucher zudem oft unverständlich sind, zeigt eine groß angelegte, weltweite Studie mit über 21.000 Verbrauchern: Etwa $90 \%$ lesen die Informationen, aber für mehr als die Hälfte der Probanden bleiben die Informationen unverständlich (ACNIELSEN 2005). Die Frage bleibt offen, ob dies auch bei Verkehrsbezeichnungen, Produkt- und Zutatenabbildungen und Clean Labeln der Fall ist.

Hinsichtlich der Schlüsselinformation „Produktname“ zeigt eine Studie von OAKES und SLOTTERBACK (2001), dass Produktnamen Erwartungen hervorrufen, die nicht immer mit der Zutatenliste zusammenpassen. Zur Bedeutung des Produktnamens gibt es eine weitere Studie von IRMAK et al. (2011): Der Name eines Lebensmittels beeinflusst die Wahrnehmung des Gesundheitswertes. Demnach liefert der Produktname für Verbraucher offensichtlich wichtige Informationen, die die Kaufentscheidung bzw. den Verzehr beeinflussen können.

Zur Verbraucherwahrnehmung von Clean Labeln konnten nach intensiver Recherche in der Literatur keine wissenschaftlichen Veröffentlichungen im Marketingbereich gefunden werden. Vereinzelt finden sich praxisnahe Veröffentlichungen (UNLAND 2011; SPILLER und ZÜHLSDORF 2012; VERBRAUCHERZENTRALE NORDRHEIN-WESTFALEN E. V. 2010), die die derzeitige Kennzeichnungspraxis von Clean Labeln kritisieren. Jedoch gibt es verschiedene wissenschaftliche Studien, die sich mit den Verbraucherpräferenzen für natürliche Produkte beschäftigen. Zum Beispiel arbeiten LI und CHAPMAN (2012) heraus, dass Verbraucher als natürlich ausgelobte Produkte mit einem besseren Geschmack verbinden und für gesünder und umweltfreundlicher halten. 
Insgesamt bleibt festzuhalten, dass in der Forschung bisher der Schwerpunkt auf Fragen der Nährwertkennzeichnung liegt. Vereinzelt finden sich Studien zur Wirkung der Aufmachung der Produktvorderseite. Die Forschungsfrage, ob Verbraucher Signalling Cues so verstehen, wie es der Zutatenliste bzw. der Zusammensetzung des Produktes zu entnehmen ist, hat bisher kaum Beachtung gefunden.

\section{$3 \quad$ Material und Methoden}

In der vorliegenden Studie wurde untersucht, wie Lebensmittelkennzeichnungen und Produktaufmachungen von Verbrauchern verstanden werden. ${ }^{7}$ Die Befragung ist im Rahmen der begleitenden Verbraucherforschung zum Internetportal Lebensmittelklarheit.de der Verbraucherzentralen und des Verbraucherzentrale Bundesverbands durchgeführt worden (BMELV 2013). ${ }^{8}$ Der hier präsentierten quantitativen Erhebung waren Fokusgruppendiskussionen zur Festlegung der Befragungsthemen und zur Hypothesengenerierung vorangestellt (ZÜHLSDORF et al. 2013).

Die Verbraucherbefragung wurde als standardisierte computergestützte persönliche Befragung (CAP-Interviews) mit 1.021 Teilnehmern mit Hilfe eines führenden Marktforschungsunternehmens durchgeführt. Die Studienteilnehmer waren deutsche Verbraucherinnen und Verbraucher ab 16 Jahren. Die Befragung fand im November und Dezember 2012 statt. Die Auswertung erfolgte mit dem Statistikprogramm IBM ${ }^{\circledR}$ SPSS, Version 20, anhand univariater Statistik.

Um eine Repräsentativität für die deutsche Bevölkerung zu gewährleisten, wurden Quoten für die Merkmale Alter, Geschlecht, Einkommen, regionale Distribution, Bildung und Haushaltsgröße entsprechend der Verteilung gesetzt. Die Quotenvorgaben wurden erfüllt. Der Altersdurchschnitt liegt bei 41 Jahren. 49,4\% der Teilnehmer sind männlich, 50,6 \% weiblich. 20,7 \% leben in Ostdeutschland, 27,5 \% im Süden, 36,1 \% in der Mitte Deutschlands und 15,8 \% der Probanden sind in Norddeutschland wohnhaft. 26,4 \% haben das Abitur oder einen Hochschulabschluss erworben. 26,5 \% leben alleine, 35,6 \% in einem zwei Personen Haushalt und $37,8 \%$ in einem Haushalt mit drei oder mehr Personen. 43,8 \% der Haushalte haben ein Nettoeinkommen von weniger als $2000 €$ zur Verfügung, 38,4 \% mehr als $2000 €$. Somit kann

\footnotetext{
${ }^{7}$ Der vollständige, detaillierte Endbericht des Projektes kann unter dem Link http://www.vzbv.de/cps/rde/xbcr /vzbv/lebensmittelkennzeichnung-ergebnisbericht-agrifood-2013.pdf eingesehen werden.

${ }^{8}$ Das Projekt wurde im Rahmen der Initiative „Klarheit und Wahrheit bei der Kennzeichnung und Aufmachung von Lebensmitteln" vom Bundesministerium für Ernährung und Landwirtschaft gefördert.
} 
die Stichprobe als näherungsweise repräsentativ für die Bevölkerung in der Bundesrepublik Deutschland angesehen werden (STATISTISCHES BunDESAMT 2012).

\section{$4 \quad$ Rechtliche Grundlagen für die Fallgruppen}

Dieses Kapitel liefert essenzielle Hintergrundinformationen, um die Problematik der derzeitigen Kennzeichnungspraxis zu verstehen. Für die drei im Rahmen des vorliegenden Beitrages dargestellten Fallgruppen gibt es keine eindeutige rechtliche Kennzeichnungsregelung. Zum Teil werden in der Praxis die Vorgaben der deutschen Lebensmittelbuchkommission herangezogen, in der Wirtschaft, Lebensmittelüberwachung, Wissenschaft und Verbraucherschaft paritätisch beteiligt sind. Allerdings sind diese Regelungen teilweise umstritten.

\section{Fallgruppe 1: Verkehrsbezeichnungen}

Im Folgenden werden die Befragungsergebnisse $\mathrm{zu}$ drei Produkten (Geflügelwurst, Kalbswurst, alkoholfreies Bier) als Fallbeispiele für das Verbraucherverständnis von Verkehrsbezeichnungen herangezogen. Diese Erzeugnisse wurden ausgewählt, da sie in der Vergangenheit mehrfach in der (Fach-)Öffentlichkeit kontrovers diskutiert wurden.

Das erste Produktbeispiel für die Gruppe Verkehrsbezeichnungen ist Geflügelwurst, mit dem das Kennzeichnungsfeld Tierartenkennzeichnung thematisiert wird. Dabei geht es insbesondere darum, inwieweit die als Zutat verwendete Fleischsorte namensgebend für das Produkt ist und wie bei der Verarbeitung mehrerer Fleischsorten zu verfahren ist. In der Praxis findet bei der Bezeichnung Geflügelwurst der generelle Leitsatz für Fleisch und Fleischerzeugnisse Nr. 2.11 der Lebensmittelbuchkommission Anwendung: Steht kein Hinweis einer Tierart in der Verkehrsbezeichnung, so besteht das Produkt aus Rind- und / oder Schweinefleisch. Verwenden Hersteller außer Schweine- und Rindfleisch eine weitere oder ausschließlich eine andere Fleischsorte, so ist dies in der Verkehrsbezeichnung durch die Nennung der Tierart kenntlich zu machen.

Das zweite Produktbeispiel ist Kalbswurst. Nach den Leitsätzen des Deutschen Lebensmittelbuchs für Fleisch und Fleischerzeugnisse (2.11) darf ein Hersteller in der Verkehrsbezeichnung den Begriff „Kalb-“ verwenden, wenn der Fleischanteil des Produktes min. 15 \% Kalbund / oder Jungrindfleisch enthält.

Das dritte Beispiel ist alkoholfreies Bier. Die Verwendung der Kennzeichnung ,alkoholfreies Bier“ ist in Deutschland gesetzlich nicht speziell geregelt. Allgemein muss der Alkoholgehalt in einem Getränk in Deutschland erst angegeben werden, wenn dieser über 1,2 Vol.-\% liegt ( 3 Abs. 1 S. 5 LMKV). Für die Feststellung der Verkehrsbezeichnung greift in diesem 
Fallbeispiel die verkehrsübliche Bezeichnung, wie sie der zuständige Wirtschaftsverband vertritt: Der Deutsche Brauer-Bund orientiert sich an der WeinV (DEUTSCHER BRAUER-Bund 2013). Demnach darf ein Wein als alkoholfrei deklariert werden, wenn er max. 0,5 Vol.-\% enthält ( $\$ 47$ Abs. 1 S. 2 WeinV). Zudem verweist der Deutsche Brauer-Bund darauf, dass die Bezeichnung alkoholfreies Bier in anderen europäischen Ländern erlaubt ist. In $\S 47$ der WeinV wird allerdings auch der Begriff ,alkoholreduziert“" verwendet.

\section{Fallgruppe 2: Produktnamen und -bilder in Zusammenhang mit der Zutatenliste}

Durch Produktnamen und -bilder auf der Verpackungsvorderseite wollen Hersteller Verbrauchern den Inhalt einer Verpackung und die Beschaffenheit des Produktes vermitteln. Hierbei geht es um die Frage, ob die durch den Produktnamen und die visuelle Aufmachung der Verpackung herausgehobenen Zutaten von Verbrauchern im Produkt selbst erwartet oder als Symbole für eine Geschmacksrichtung interpretiert werden. Die abgefragten Beispiele wurden herstellerneutral als Dummies gestaltet. Thematisch greifen sie typische Fallkonstellationen auf, wie sie im Portal Lebensmittelklarheit.de diskutiert werden.

Als lebensmittelrechtliche Grundlage kann hier allgemein $\S 8$ der LMKV (ab 13.12.2014 Art. 9 Abs. 1 lit. d LMIV), der die Mengenkennzeichnung regelt, herangezogen werden. Hiernach müssen Mengen bestimmter Zutaten im Zutatenverzeichnis angegeben werden. So ist in den drei im Rahmen der vorliegenden Studie behandelten Produktbeispielen Beerenfruchtschnitte, Thai-Food Ente und gefüllte Frischnudeln durch Bilder auf der Produktvorderseite die Mengenangabe der abgebildeten Zutaten in der Zutatenliste erforderlich. Ein weiteres Produktbeispiel ist Ziegenfrischkäse. Hier kommt zusätzlich die KäseVO zur Anwendung. Für den Begriff Käse ist dort geregelt, dass Milcherzeugnisse ganz oder teilweise durch entsprechende Erzeugnisse aus Ziegenmilch ersetzt werden können ( 1 Abs. 2 KäseVO). Das letzte Fallbeispiel ist ein Wellness Wasser, auf dessen Etikett Früchte abgebildet sind. Zur Beurteilung der verwendeten Abbildung werden die Leitsätze der Lebensmittelbuchkommission für Erfrischungsgetränke, insbesondere I C 3, herangezogen. Naturgetreue Abbildungen von Früchten sind demnach auch ohne Fruchtsaftanteil im Getränk möglich.

\section{Fallgruppe 3: Clean Label}

In den letzten Jahren hat die Kennzeichnung von Lebensmitteln mit sog. Clean Labeln deutlich zugenommen, womit Hersteller und Produzenten den Verzicht des Einsatzes von Verbrauchern als kritisch betrachteten Zusatzstoffen ausloben. Im dritten Abschnitt wird daher das Verbraucherverständnis von Clean Labeln an Hand der Beispiele „Cappuccino ungesüßt“, 
„Erdbeerjoghurt ohne Farbstoffe“ und des Claims „frei von Geschmacksverstärkern“ analysiert. Rechtlich sind diese Aussagen zulässig, wenn i. S. v. § 11 LFGB keine Irreführung vorliegt. Detaillierte Regelungen gibt es nicht. Maßgeblich ist die Auffassung durch den „durchschnittlich informierten, situationsadäquat aufmerksamen und verständigen Verbraucher“" (z. B. GRUR Int. 1999, 354ff, „Sektkellerei Kessler“).

\section{$5 \quad$ Verbrauchereinschätzung ausgewählter Kennzeichnungen}

\subsection{Fallgruppe 1: Verkehrsbezeichnungen}

Verkehrsbezeichnungen sollen Verbrauchern Aufschluss über die Beschaffenheit eines Produktes liefern (Art. 17 Abs. 1 LMIV). Die Frage ist, ob die bisher verwendeten Bezeichnungen dem Verbraucherverständnis gerecht werden, da es hierzu keine empirischen Untersuchungen gibt. Die ersten beiden Fallbeispiele beziehen sich auf die Verkehrsbezeichnungen Geflügel- und Kalbswurst. Erwarten Verbraucher die Nennung aller verarbeiten Fleischarten in der Produktbezeichnung oder schließen sie auf Grund des Zutatenverzeichnisses auf die Tierarten? Um mögliche tierartenspezifische Unterschiede zu erfassen, wurde die Stichprobe für die Untersuchung des Kennzeichnungsfeldes Tierartenkennzeichnung in zwei SubSamples unterteilt. 512 Teilnehmer wurden zu Geflügelwurst befragt, 509 Probanden bekamen das Produktbeispiel Kalbswurst vorgelegt. Die Fragestellungen waren für die beiden Produkte analog.

Das erste Fallbeispiel betrifft die Verkehrsbezeichnung Geflügelwurst. In 2011 hat die Stiftung Warentest Geflügelfleischerzeugnisse getestet. Dazu wurden 207 Geflügelfleischerzeugnisse gekauft und anhand der Verkehrsbezeichnung und der Zutatenliste bewertet. Bei 53 Produkten können Verbraucher erst beim Betrachten der Zutatenliste erkennen, dass die Wurst neben Geflügel- auch Schweine- oder Rindfleisch enthält (STIFTUNG WARENTEST 2011).

Die Verbraucher wurden gefragt, wie sie die Verkehrsbezeichnung Geflügelwurst auffassen. Unter einer Geflügelwurst verstehen demnach 73,6\% der Verbraucher, dass für eine Wurst mit dieser Bezeichnung ausschließlich Geflügelfleisch verarbeitet wird. 22,7 \% sind der Auffassung, dass das Produkt sowohl Schweine- als auch Geflügelfleisch enthält. 3,7 \% sind sich nicht sicher, wie die Bezeichnung zu verstehen ist.

Zusätzlich zur Geflügelwurst wurde „Kalb“ als Tierart gewählt, um ggf. Unterschiede zwischen der Kennzeichnung verschiedener Tierarten aufdecken zu können. Um die Produktbeispiele einheitlich und verständlich zu gestalten, wurde der Begriff Kalbswurst gewählt. In 
der Praxis finden sich neben Kalbswurst verschiedene andere Produktbezeichnungen mit der Tierart „Kalb“.

Die praktische Umsetzung durch die Hersteller bei Kalbsleberwurst, Kalbfleisch-Leberwurst oder Kalbsbratwürsten weicht deutlich voneinander ab. Während Kalbfleisch-Leberwurst und Kalbsleberwurst meist zwischen $15 \%$ und $17 \%$ Kalbfleisch enthalten, fallen die Kalbfleischanteile bei Kalbsbratwurst mit $60 \%$ bis z. T. $100 \%$ Kalbfleisch im Fleischanteil deutlich höher aus.

Die Befragung der Probanden zur Kalbswurst erfolgte analog zu Geflügelwurst. Im Ergebnis erwarten $66,4 \%$ der Teilnehmer von einer Kalbswurst, dass ausschließlich Kalbfleisch im Produkt verarbeitet wird. 30,3\% sind der Auffassung, dass eine Mischung aus Schweine- und Kalbfleisch vorhanden ist. 3,3\% geben an, dass sie sich nicht sicher sind, wie die Produktbezeichnung zu verstehen ist.

Ein anderes kontrovers diskutiertes Fallbeispiel ist alkoholfreies Bier. Erwarten Verbraucher in einem als akoholfrei deklarierten Bier einen Restalkoholgehalt oder gehen sie davon aus, dass das Bier gänzlich alkoholfrei ist? In der Praxis werden Biere mit z. T. sehr unterschiedlichen Alkoholgehalten als alkoholfrei deklariert und vermarktet. Die Spanne umfasst sowohl Produkte wie die Marke Warsteiner Premium alkoholfrei der Warsteiner Brauerei, das gar keinen Alkohol enthält (0,0 Vol.-\%), als auch Biere mit höheren Alkoholgehalten wie z. B. das Clausthaler Classic Premium alkoholfrei der Radeberger Gruppe mit einem Alkoholgehalt von 0,45 Vol.- $\%$.

Um Erkenntnis darüber zu erlangen, wie Verbraucher die Kennzeichnung alkoholfreies Bier verstehen, sollten die Probanden in der Befragung angeben, ob sie Alkohol in einem so gekennzeichneten Bier erwarten. Auf einer fünfstufigen Likert Skala von „Ja, auf jeden Fall“ bis „Nein, auf keinen Fall“ wurde das Verbraucherverständnis mit zwei Items abgefragt. Dabei wurden die Antwortmöglichkeiten randomisiert vorgelegt. 69,2\% der Teilnehmer verstehen unter der Kennzeichnung alkoholfreies Bier, dass das Getränk keinen Alkohol enthält. Nur 30,3\% vermuten, dass das Bier eine gewisse Menge an Alkohol enthält. ${ }^{9}$

\footnotetext{
${ }^{9}$ Die genaue Verteilung der Antworten dieses und der folgenden Fallbeispiele können den Tabellen im Anhang entnommen werden.
} 


\subsection{Fallgruppe 2: Produktnamen und Zutaten- bzw. Produktabbildungen ${ }^{10}$}

Die zweite Fallgruppe beschäftigt sich mit der Frage nach demVerbraucherverständnis, das sich aus der Kombination von Abbildungen und Produktbezeichnungen auf der Produktvorderseite ergibt. Interpretieren Verbraucher die Nennung von Lebensmitteln im Produktnamen und deren visuelle Darstellung als Hinweis auf die Geschmacksrichtung eines Produktes oder als Hinweis auf die Produktbeschaffenheit? Zur Untersuchung dieser Fragestellung wurde den Probanden jeweils zunächst ein Produktdummy mit Ansicht der Schauseite einer Lebensmittelverpackung gezeigt und anschließend die zugehörige Zutatenliste. Die dargestellten Fallbeispiele wurden in der Befragung randomisiert vorgelegt. Zur Erhebung des Verbraucherverständnisses wurden die Probanden gefragt, ob Bilder und Bezeichnung zu den Produkten passen (fünfstufige Likert Skala von „Ja, auf jeden Fall“ bis „Nein, auf keinen Fall“).

Derzeit sind am Lebensmittelmarkt mehrere Beeren-Fruchtschnitten verfügbar, die oft zu einem Großteil aus Sultaninen oder Apfelpulver bestehen und Beeren in geringen Umfang enthalten. Auf der Produktvorderseite sind allerdings ausschließlich oder dominierend Beeren abgebildet. 42,8 \% der Verbraucher finden die Bilder der gezeigten Beerenfruchtschnitte passend zu der vorgelegten Zutatenliste. 44,8 \% lehnen dies ab. 38,7 \% finden, dass die Produktbezeichnung zu den Zutaten passt. Hier lehnen $43 \%$ der Konsumenten ab, dass die Produktbezeichnung passt.

Das nächste Produktbeispiel thematisiert den Produktbereich Erfrischungsgetränke. Mineralwässer mit Fruchtgeschmack werden bei Verbrauchern immer beliebter. Stiftung Warentest hat 25 Wässer mit Geschmack getestet. Obwohl alle Hersteller mit Früchten auf der Flasche werben, ist fast immer Aroma im Wasser enthalten (STIFTUNG WARENTEST 2013). Bei dem vorgelegten Produktdummy sind vorne auf der Flasche deutlich sichtbar Lychees und Guaven abgebildet. Die Zutatenliste legt offen, dass der Geschmack durch Trauben und natürliches Aroma erzielt wird. 35,4\% stimmen der Aussage zu, dass die Zutatenabbildungen zu den angegebenen Zutaten passen, 49,1\% lehnen dies ab. 32,2\% der Befragten finden, dass die Produktbezeichnung „Wellness-Lychee-Guave“ zu den angegeben Zutaten passt. Mehr als die Hälfte $(51,3 \%)$ findet die Produktbezeichnung unpassend.

\footnotetext{
${ }^{10}$ Die in der Befragung verwendeten Produkt- bzw. Zutatenbilder und Zutatenlisten können ebenfalls dem Endbericht entnommen werden (http://www.vzbv.de/cps/rde/xbcr/vzbv/lebensmittelkennzeichnung-ergebnisberichtagrifood-2013.pdf).
} 
Das nächste Produktbeispiel bezieht sich auf Instant-Produkte. Auf dem Lebensmittelmarkt finden sich Instant-Nudelsuppen, die mit Abbildungen von Fleisch auf der Produktvorderseite werben, aber lt. Zutatenliste ausschließlich Aroma im Produkt enthalten ist. Exemplarisch dafür wurde eine Instant-Nudelsuppe mit der Bezeichnung Thai-Food Ente getestet. Hier finden 33,5\%, dass die Bilder und die Zutaten zusammen passen. Mit der Produktbezeichnung sind 26,5\% einverstanden. Es zeigt sich insgesamt, dass bei diesem Produktbeispiel mehr als die Hälfte der Probanden (53,1\% und 54,5\%) Bezeichnung und Bebilderung als nicht passend bewerten.

Ein weiteres Beispiel betrifft die Tierartenkennzeichnung von Milchprodukten, wenn andere Milchsorten als Kuhmilch verwendet werden. Angeboten werden auf dem Markt z. B. Frischkäsesorten, die zu $100 \%$ aus Ziegenmilch hergestellt werden, andere haben nur einen Anteil von $5 \%$. Dabei ist auf der Produktvorderseite häufig eine Ziege abgebildet; die Milchkuh, die den größeren Milchanteil liefert, jedoch nicht. Im vorliegenden Fall versucht der Hersteller dies durch den Zusatz „mit“ mildem Ziegenkäse zu kennzeichnen. Mit 60,9 \% der Teilnehmer ist weit mehr als die Hälfte mit der Bebilderung einverstanden. Weiterhin sind 61,7 \% der Meinung, dass die Produktbezeichnung mit der Zutatenliste im Einklang steht.

Das letzte Produktbeispiel sind gefüllte Frischnudeln (Triangolini) mit Steinpilzen und Trüffeln. Auf dem Lebensmittelmarkt werden verschiedene Produkte (gefüllte Nudeln, Risottomischungen und Fertigsuppen) angeboten, die im Produktnamen Steinpilz oder Trüffel enthalten. Die Zutatenliste legt offen, dass diese Zutaten aber nur einen ausgesprochen geringen Anteil am Gesamtprodukt ausmachen.

Die Mittelwerte $(0,04$ und 0,00) bei beiden Statements zeigen an, dass sich keine klare Meinung zur Produktaufmachung herausstellen lässt. 43,5 \% finden, dass die Verpackung zu den Zutaten passt, 37,2 \% finden das Gegenteil. Mit der Produktbezeichnung sind 41,8 \% einverstanden, 39,9\% denken, dass die Bezeichnung nicht passt.

\subsection{Fallgruppe 3: Clean Label}

Die letzte Fallgruppe thematisiert das Kennzeichnungsfeld der Clean Label bzw. Aussagen auf den Verzicht bestimmter Inhaltsstoffe. Die Zunahme dieser Kennzeichnungspraxis ist dem Verbraucherwunsch nach natürlichen Lebensmitteln geschuldet. Es stellt sich die Frage, wie weitreichend das Verbraucherverständis so gekennzeichneter Produkte bei der derzeitigen Kennzeichnungspraxis ist. Die von den Probanden zu bewertenden Aussagen zu den Fallbeispielen wurden jeweils randomisiert vorgelegt und das Verbraucherverständnis auf einer fünfstufigen Likert Skala von „Ja, auf jeden Fall“ bis „Nein, auf keinen Fall“ abgefragt. 
Ernährungsempfehlungen raten von zu hohem Zuckerkonsum ab. Daher bieten viele Hersteller „Cappuccino ungesüßt“ an, um ernähungsbewusste Verbraucher anzusprechen. In der Health Claims Verordnung (HCVO) sind die Begriffe „zuckerarm“, „zuckerfrei“ und „ohne Zuckerzusatz“ geregelt. Nach der HCVO sollten diese Regelungen auch für Angaben gelten, die für Verbraucher dieselbe Bedeutung haben, wie es bei „ungesüßtem“ Cappuccino der Fall sein kann. Im Handel erhältlichem „ungesüßten“ Cappuccino sind oft für einen zuckrigen Geschmack andere süßende Zutaten wie Glukosesirup, Lactose, Magermilchpulver, Maltodextrin oder Süßmolkenpulver zugefügt. Beim Beispiel Cappuccino ungesüßt wurden die Probanden gefragt, wie sie den Aufdruck ,ungesüßt“ verstehen. 80,6\% geben an, dass sie glauben, dass dem Produkt kein Zucker zugesetzt ist, nur 5,8 \% glauben dies nicht. 54,3\% sind der Meinung, dass das Produkt insgesamt wenig Zucker enthält, hier denken 29,4 \%, dass dies nicht darunter zu verstehen ist. Weiterhin erwarten $74,8 \%$, dass dem Produkt keinerlei süßende Zutaten zugesetzt sind, nur 9,6\% erwarten diese nicht.

Ein weiterer, oft verwendeter Claim lautet „ohne Farbstoffe“. Eine bundesweite Markterhebung der Verbraucherzentralen (VERBRAUCHERZENTRALE NORDRHEIN-WESTFALEN 2010), bei der 272 Auslobungen von Clean Labeln bewertet wurden, zeigt, dass in $62 \%$ der Fälle bei der Verwendung des Labels „ohne Farbstoffe“ oder „ohne künstliche Farbstoffe“ mit anderen Zutaten (z. B. Rote Bete) gefärbt wurde. Bei dem Produktbeispiel Erdbeerjoghurt mit dem Claim ohne Farbstoffe denkt der Großteil der Befragten (80,1\%), dass dem Produkt keine Farbstoffe zugesetzt sind. Bei dem Zusatz von färbenden Lebensmitteln ist sich ein Viertel $(25 \%)$ der Probanden unsicher, aber mehr als die Hälfte $(57,6 \%)$ glaubt, dass auch keine färbenden Lebensmittel enthalten sind. Weiterhin denken 60,4 \%, dass die Farbe des Joghurts nur aus Erdbeeren stammt. Bei diesem Statement ist sich knapp ein Viertel $(21,9 \%)$ der Verbraucher unsicher.

Das letzte Fallbeispiel fragt das Verständnis der Aussage „frei von Geschmacksverstärkern“ ab. Bei der bereits oben erwähnten Erhebung der Verbraucherzentrale (VERBRAUCHERZENTRALE NORDRHEIN-WESTFALEN 2010) enthalten $92 \%$ der untersuchten Produkte, die das Label „ohne Geschmacksverstärker“ tragen, potenziell geschmacksverstärkende Zutaten mit einem hohen Anteil an Aminosäuren. Konkret wurde in der Befragung getestet, ob die Verbraucher bei der Aufschrift „frei von Geschmacksverstärkern“ erwarten, dass Hefeextrakt, Sojasauce, Aroma oder Parmesankäse enthalten ist (Statement: „Ich verstehe unter dem Aufdruck „frei von Geschmacksverstärkern“, dass das Produkt ... kein XX enthält“). Dabei wurden die Probanden zuvor informiert, dass Hersteller für einen intensiveren Geschmack manchmal Zutaten einsetzen, die natürlicherweise freie Glutaminsäure enthalten. Die Mittel- 
werte (0,46 für Hefeextrakt; 0,42 für Sojasauce; 0,71 für Aromen; 0,39 für Parmesankäse) zeigen bereits an, dass die Mehrheit der Probanden ein geschmackliches Tuning bei allen vier Substituten nicht erwartet. Im Detail sind dies bei Hefeextrakt 49,9\%, bei Sojasauce 48,9\%, bei Aromen 63,87\% und bei Parmesankäse 48,7\%. Zu beobachten ist auch, dass der Anteil unsicherer Konsumenten relativ hoch ausfällt: Jeweils $30 \%$ sind sich bei Hefeextrakt und Sojasauce unsicher, bei Aromen 18,5 \% und bei Parmesan 27,8 \%.

\section{Diskussion der Fallgruppen}

Insgesamt lässt sich festhalten, dass über alle drei untersuchten Fallgruppen hinweg mehr als die Hälfte der Teilnehmer die Produkte anders interpretieren, als es Hersteller durch Verkehrsbezeichnung, Namensgebung, Bebilderung oder Clean Label darstellen. Eine Ausnahme bildet das Produkt Ziegenkäse: Hier sind jeweils über $60 \%$ der Befragten der Meinung, dass die Zutatenabbildungen auf der Verpackung und der Produktname zu der Zutatenliste passen. Dies könnte dadurch erklärt werden, dass das Produkt selbst als Frischkäse deklariert ist und den relativierenden Zusatz „,mit“ mildem Ziegenkäse trägt. Offensichtlich trägt die sprachliche Präzisierung zu einem besseren Produktverständnis bei.

Für die Fallbeispiele Geflügelwurst und Kalbswurst kann festgestellt werden, dass der Großteil der Verbraucher an Hand der Verkehrsbezeichnung schlussfolgert, von welchen Tierarten Fleisch in einem Fleischprodukt verarbeitet worden ist. Aus Verbrauchersicht ist diese Information nicht unwesentlich. Neben religiösen Gründen (vgl. ROTH 2008) können Verbraucher auch aus gesundheitlichen Gründen bestimmte Fleischarten bevorzugen, bspw. auf Grund eines niedrigen Fettgehaltes (HAUNER 2013).

Eine vergleichbare Problematik zeigt sich für das Fallbeispiel alkoholfreies Bier. Eine klare Mehrheit der Verbraucher versteht die Kennzeichnung von ,alkoholfreiem Bier“ so, dass es gar keinen Alkohol enthält. Für verschiedene Verbrauchergruppen, wie abstinente Alkoholiker oder Konsumenten, die aus religiösen Gründen auf Alkohol verzichten, ist eine korrekte und eindeutige Deklaration wünschenswert.

Bei der Abfrage der Kongruenz zwischen Produktnamen und Produkt- bzw. Zutatenabbildungen auf der einen und der Zutatenliste auf der anderen Seite zeigt sich, dass mehr als die Hälfte der Verbraucher der Auffassung ist, dass die Aufmachung der Produktvorderseite nicht angemessen ist, wenn man die Zusammensetzung des Produktes berücksichtigt. Damit rufen Bilder und Namen bei Verbrauchern offensichtlich Assoziationen hervor, die sich nicht mit der Zutatenliste decken. Dies kann der knappen Zeit beim Einkaufen 
(SCHRANKEL 2005) geschuldet sein, sodass sich Verbraucher mehr auf die Verpackungsvorderseite als auf die -rückseite konzentrieren. So entsteht Potenzial für Täuschungsempfinden, wenn die Rückseite nicht aufmerksam gelesen wird.

Bei Clean Labeln ist die Verbrauchererwartung bei allen drei Beispielen (ungesüßt, ohne Farbstoffe, frei von Geschmacksverstärkern) bezogen auf den Verzicht ähnlich wirkender Ersatzstoffe relativ weitgehend und weitgehend unabhängig von den lebensmittelrechtlichen Klassifizierungen. Zugleich zeigt sich, dass ein nicht unerheblicher Anteil der Probanden über den Einsatz der abgefragten Zusätze verunsichert ist (13\% und mehr bei der Antwortoption „Bin mir unsicher“). Bei den geschmacksverstärkenden Zusätzen ist die Verunsicherung besonders ausgeprägt: Bei Hefeextrakt und Sojasauce sind sich jeweils $30 \%$ der Befragten unsicher, ob bei der Kennzeichnung frei von Geschmacksverstärken diese Zutaten enthalten sein können. Um Clean Label umfassend interpretieren zu können, bräuchten Verbraucher tiefer gehendes Detailwissen, was wenig realistisch erscheint.

Es muss offen bleiben, ob die Verunsicherung das Ergebnis geringer Informationsbemühungen der Konsumenten oder wenig aufklärender Kommunikationsmaßnahmen der Hersteller ist (KÜHL et al. 2013). Jedenfalls führt die derzeitige Kennzeichnungspraxis - obwohl lebensmittelrechtlich unter Umständen konform - wie aufgezeigt zu einem hohen Irreführungspotenzial und zahlreichen negativen Medienberichten (BRÜHL 2011, KIRSCHEY und HAUER 2012, KUHLMANN 2012).

\section{$7 \quad$ Schlussfolgerungen und Limitationen}

Verbraucherpolitik soll den Konsumenten vor Irreführung und Täuschung schützen (BMELV o. J.; LMIV). Auch wenn ein Produkt im Zutatenverzeichnis viele detaillierte Informationen liefert, so haben bzw. nehmen sich viele Verbraucher realiter kaum die Zeit, diese bei jedem Produkt zu lesen, zu verstehen und miteinander zu vergleichen. Die in 2014 in Kraft tretende Lebensmittelinformationsverordnung der Europäischen Union weist deshalb explizit darauf hin, dass Lebensmittel klar und verständlich gekennzeichnet sein müssen (VO 1169/2011/EG, Erwägungsgrund 9; Art. 7 Abs. 2 VO 1169/2011/EG).

Um dem Verbraucher zu einer rationaleren Entscheidung zu verhelfen, ist es vor dem Hintergrund des faktischen Verbraucherverhaltens wichtig, auf der Produktverpackung prägnante, entscheidende Informationen zu liefern (DANTAS et al. 2004, VAN HERPEN und VAN TRIJP 2011, ZÜHLSDORF et al. 2013). Die Marketingforschung konzentriert sich hier bisher fast ausschließlich auf Food Labelling - jedoch nicht auf die zunehmende Kennzeichnungspraxis des Clean Labellings - (z. B. HARPER et al. 2007) und nährwert- (z. B. DE LA HUNTY et al. 
2014) bzw. gesundheitsbezogene Angaben (BIALKOVA und VAN TRIJP 2010). Schlüsselinformation können aber auch die Verkehrsbezeichnung, Produkt- bzw. Zutatenbilder und -namen oder Clean Label sein. Aus den vorliegenden Ergebnissen lässt sich schlussfolgern, dass deutsche Verbraucher die Verpackungsaufmachung der Fallgruppen nicht ohne weitere Erklärung so verstehen, wie es der Produktzusammensetzung entspricht. Die erste Fallgruppe der Verkehrsbezeichnungen zeigt auf, dass die Verbraucherauffassung nicht mit der jeweiligen Verkehrsbezeichnung in Kongruenz steht. Stellt man Verbrauchern Produkt- bzw. Zutatenbilder und -namen direkt neben die Zutatenliste, wird deutlich, dass bis auf die Ausnahme des Ziegenkäses jeweils weniger als die Hälfte der Verbraucher der Auffassung ist, dass die Bilder und Namen der Produkte bei der gezeigten Zutatenliste angemessen sind.

Die fehlende Anpassung der Produktaufmachung an die Verbrauchererwartungen führt in den drei untersuchten Fallgruppen zu Fehleinschätzungen bei Verbrauchern und birgt die Gefahr, dass Verbraucher sich getäuscht fühlen, sobald Ihnen ihre Fehleinschätzung bewusst wird. Die Lebensmittelkennzeichnung muss die Interessen von Verbrauchern, Produzenten und Herstellern gleichermaßen berücksichtigen. Im Zweifelsfall wiegen jedoch die Verbraucherinteressen auf Grund des Schutzes der Verbraucher vor Irreführung und Täuschung stärker als die der Wirtschaft (HOHMANN 1994). Die Hersteller sollten dabei ebenfalls ein Interesse daran haben, das Vertrauen ihrer Kunden zu erlangen und Transparenz zu schaffen.

\section{Literatur}

ACNiElsen (2005): The Nutrition-CONSCIOUs GlobAl ShOPPER - CONSUMER AtTitudes TOWARDS NUTRITION LABEL ON FOOD PACKAGING IN EUROPE. AUSTRALIA.

AKERLOF, G. A. (1970): THE Market for "Lemons": Qualitative Uncertainty and the Market Mechanism. In: Quarterly Journal of Economics. 84 (3): 488-500.

BIALKOVA, S. und VAN TRIJP, H. (2010): What determines consumer attention to nutrition labels? In: Food Quality and Preference 21: 1042-1051.

BMELV BUNDESMINISTERIUM FÜR ERNÄHRUNG, LANDWIRTSCHAFT UND VERBRAUCHERSCHU TZ (2013): BMELV-Initiative „Klarheit und Wahrheit“. http://www.bmelv.de/Shared Docs/Standardartikel/Ernaehrung/SichereLebensmittel/Kennzeichnung/KlarheitUndWa hrheit/KlarheitUndWahrheitBeiLebensmitteln.html. Abgerufen am 7. August 2013.

BMELV BUNDESMINISTERIUM FÜR ERNÄHRUNG, LANDWIRTSCHAFT UND VERBRAUCHERSCHU TZ (o. J.): Verbraucherschutz und Informationsrechte. http://www.bmelv.de/DE/EuropaInternationales/Verbraucherpolitik/verbraucherpolitik_node.html. Abgerufen am 26. September 2013.

BRÜHL, J. (2011): Irreführende Lebensmittelpackungen - Erdbeeren, mit Datteln gestreckt. Süddeutsche.de. Wirtschaft. http://www.sueddeutsche.de/wirtschaft/irrefuehrendelebens 
mittelpackungen-erdbeeren-mit-datteln-gestreckt-1.1129249. Abgerufen am 15. Oktober 2013.

Cowburn, G. und Stockley, L. (2004): Consumer understanding and use of nutrition labelling: a systematic review. In: Public Health Nutrition 8 (1): 21-28.

Dantas, M. I. S., Minim, V. P. R., Rosires, D. und Puschmann, R. (2004): The effect of packaging on the perception of minimally processed products. In: Journal of International Food \& Agribusiness Marketing. 16 (2): 71-83.

De la Hunty, A., Ashwell, M., Arens, U., Gibson, S. und Sadler, M. (2014): Authorised Health Claims May Not Help Consumers to Choose a Healthy Diet. In: Annals of Nutrition and Metabolism 64 (1): 1-5.

DEUTSCHER BRAUER-BUND E. V. (2013) Alkoholfreies Bier. http://www.brauerbund.de/aktuell/

alkoholfreies-bier.html. Abgerufen am 25. Februar 2013.

FeuneKes, G. I. J., GortemaKer, I. A., Willems, A. A., LiOn, R. und VAN DEN Kommer, M. (2008): Front-of-pack nutrition labelling: Testing effectiveness of different nutrition labelling formats front-of-pack in four European countries. In: Appetite 50: 57-70.

FERNQUIST, F. und EKELUND, L. (2014): Credence and the effect on consumer liking of food A review. In: Food Quality and Preference, 32: 340-353.

Grunert, K. G., Fernández-Clemín, L., Wills, J. M., Storcksdiek, S. und Nureeva, L. (2010): Use and understanding of nutrition information on food labels in six European countries. In: Journal of Public Health, 18 (3): 261-277.

Harper, L., Souta, P., Ince, J. und Mckenzie, J. (2007): Food Labelling Consumer Research: What Consumers Want. A Literature Review. Food Standards Agency.

HAuner, H. (2013): Ernährungstherapie des Typ-2-Diabetes. In: Der Diabetologe 9 (5): 405416.

HoHMANN, G. (1994): Die Verkehrsauffassung im deutschen und europäischen Lebensmittelrecht. P.O.C.-Verlag, Bayreuth.

Irmak, C., VAllen, B. und Robinson, S. R. (2011): The Impact of Product Name on Dieters' and Nondieters' Food Evaluations and Consumption. In: Journal of Consumer Research 38 (2): 390-405.

KIRMANI, A. und RAO, A. R. (2000): No pain, no gain: A critical review of the literature on signaling unobservable product quality. In: Journal of Marketing 64 (2): 66-79.

KIRSCHEY, R. und HAUER, T. (2012): Traumfabrik Essen - Das Märchen vom natürlichen Lebensmittel. 3 sat. Scobel. http://www.3sat.de/page/?source=/scobel/160819 /index.html. Abgerufen am 15. Oktober 2013.

KolODINSKY, J. (2012): Persistence of Health Labeling Information Asymmetry in the United States: Historical Perspectives and Twenty-First Century Realities. In: Journal of Macromarketing 32 (2): 193-207.

KÖNIGSTORFER, J. und GRÖPPEL-KLEIN, A. (2012): Wahrnehmungs- und Kaufverhaltenswirkungen von Nährwertkennzeichen auf Lebensmitteln. In: Marketing ZFP - Journal for Research and Management 34 (3): 213-226.

Kroeber-RIEL, W. und Esch, F.-R. (2004): Strategie und Technik der Werbung: Verhaltenswissenschaftliche Ansätze. Kohlhammer GmbH, Stuttgart.

KÜHL, R., MÄNCHER, A., und PIPER, A. (2013): Lebensmittelklarheit.de und Verbraucherpolitik - oder wie viel Schutz verdient Ignoranz?. Diskussionspapier Nr. 2013-1. Universität Gießen. 
Kuhlmann, S. (2012): Irreführende Bezeichnungen von Lebensmitteln. Deutschlandfunk. Umwelt und Verbraucher. http://www.dradio.de/dlf/sendungen/umwelt/1960820/. Abgerufen am 15. Oktober 2013.

LI, M., und CHAPMAN, G. B. (2012): Why do people like natural? Instrumental and ideational bases for the naturalness preference. In: Journal of Applied Social Psychology, 42 (12), 2859-2878.

OAKes, M. E. und SLOTTERBACK, C. S. (2001): What's in a name? A comparison of men's and women's judgements about food names and their nutrient contents. In: Appetite 36: 29-40.

RAYNER, K. (1998): Eye movements in reading and information processing: 20 years of research. In: Psychological Bulletin, 124 (3): 372-422.

Roth, K. (2008): Chemische Produktion gemäß Koran und Thora. In: Chemie in unserer Zeit 42 (1): 42-51.

SCHRANKEL, H. (2005): Zeitverwendung der privaten Haushalte Vergleich der Zeitbudgeterhebungen 2001/02 gegenüber 1991/92. In: Seifert, W., Möhring, K., Zühlke, S., Schrankel, H.: Statistische Analysen und Studien. Band 24. http://www.it.nrw.de/statistik/analysen/stat studien/2005/band_24/schrankel_24_2005.pdf. Abgerufen am 12. März 2013.

Sørensen, H. S., Clement, J. und Gabrielsen, G. (2012): Food Labels - an Exploratory Study into Lable Information and what Consumers See and Understand. In: The International Review of Retail, Distribution and Consumer Research. 22 (1): 101-114.

SPILLER, A. und ZüHLSDORF, A. (2012): Lebensmittelzusatzstoffe und Clean Labelling: Der Verbraucher im Spannungsfeld zwischen Information und Erwartung, in: H. Wilhelm Schaumann Stiftung (Hrsg.): 24. Hülsenberger Gespräche 2012: 180-191.

StATISTisches Bundesamt (2012): Statistisches Jahrbuch. Deutschland und Internationales. Wiesbaden.

https://www.destatis.de/DE/Publikationen/StatistischesJahrbuch/Statistisches Jahrbuch2012.pdf?_blob=publicationFile. Abgerufen am 29. Juli 2013.

STIFTUNG WARENTEST (2013): Wasser mit Geschmack. http://www.test.de/Wasser-mitGeschmack-Kunstaroma-statt-Frucht-4533487-0/. Abgerufen am 05. August 2014.

STIFTUNG WARENTEST (2011): Geflügelwurst. http://www.test.de/filestore/4272420_t201109 020.pdf?path=/protected/24/71/7afdae8f-e1d4-4a9f-b692-dc2638f068adprotectedfile.pdf\&key=D674D4FF9BAC5175854255D637630E17C3A94531. Abgerufen am 1. März 2013.

UnLAND, P. (2010): Clean Labelling, in: Behr's Jahrbuch für die Lebensmittelwirtschaft, Hamburg.

VAN HeRPEN, E. und VAN TRIJP, H. C. M. (2011): Front-of-pack nutrition labels. Their effect on attention and choices when consumers have varying goals and time constraints. In: Appetite. 57 (1): 148-160.

Van Kleef, E., Van Trijp, H., Paeps, F. und FernándeZ-Celemín， L. (2008): Consumer preferences for front-of-pack calories labelling. In: Public health nutrition 11 (2): 20313.

VERBEKE, W. (2013): Food quality policies and consumer interests in the EU. 13-22. In: M. Klopčič, M., Kuipers, A. und Hocquette, J-F. (Hrsg.) (2013): Consumer attitudes to food quality products: Emphasis on Southern Europe. EAAP publication No. 133 , Wageningen. 
VERbraucherZENTRALE NordRHEIN-WestFalen E. V. (2010): „Ohne Zusatzstoffe“ - Clean Labeling: Werbeaussagen kritisch beleuchtet. http://www.vzhh.de/ernaehrung/79067 /Clean\%20Label-Bericht\%2027-9-2010.pdf. Abgerufen am 23. Juli 2014.

ZÜHLSDORF, A., NitZKO, S. und SPILlER, A. (2013): Kennzeichnung und Aufmachung von Lebensmitteln aus Sicht der Verbraucher: Empirische Untersuchungsbefunde. Begleitende Verbraucherforschung zum Internetportal www.lebenmittelklarheit.de der Verbraucherzentralen und des Verbraucherzentrale Bundesverbands, Göttingen. 
Anhang: Detaillierte Ergebnisse des Verbraucherverständnisses der Fallgruppen

Tabelle 1: Verbraucherverständnis ,alkoholfreies Bier“

\begin{tabular}{|l|c|c|c|c|c|c|c|}
\hline $\begin{array}{l}\text { Ich verstehe unter der Be- } \\
\text { zeichnung ,alkoholfreies } \\
\text { Bier“, dass das Bier.. }\end{array}$ & $\begin{array}{c}\text { Ja, auf } \\
\text { jeden } \\
\text { Fall }\end{array}$ & Ja & $\begin{array}{c}\text { Bin mir } \\
\text { un- } \\
\text { sicher }\end{array}$ & Nein & $\begin{array}{c}\text { Nein, } \\
\text { auf } \\
\text { keinen } \\
\text { Fall. }\end{array}$ & $\begin{array}{c}\text { Mittel- } \\
\text { wert }\end{array}$ & $\begin{array}{c}\text { Stand- } \\
\text { ardab- } \\
\text { weich- } \\
\text { ung }\end{array}$ \\
\hline ...keinen Alkohol enthält. & $\mathbf{4 2 , 9 \%}$ & $\mathbf{2 6 , 3} \%$ & $\mathbf{1 0 , 6} \%$ & $\mathbf{1 6 , 2} \%$ & $\mathbf{4 , 0} \%$ & $\mathbf{0 , 8 8}$ & $\mathbf{1 , 2 3 7}$ \\
\hline $\begin{array}{l}\text {...geringe Mengen Alkohol } \\
\text { enthält (bis 0,5 Vol.-\%). }\end{array}$ & $\mathbf{8 , 9 \%}$ & $\mathbf{2 1 , 4} \%$ & $\mathbf{1 6 , 7} \%$ & $\mathbf{3 1 , 6 \%}$ & $\mathbf{2 1 , 4} \%$ & $\mathbf{- 0 , 3 5}$ & $\mathbf{1 , 2 7 3}$ \\
\hline
\end{tabular}

Tabelle 2: Verbraucherverständnis Beeren-Fruchtschnitte

\begin{tabular}{|l|c|c|c|c|c|c|c|}
\hline & $\begin{array}{c}\text { Ja, auf } \\
\text { jeden } \\
\text { Fall }\end{array}$ & Ja & $\begin{array}{c}\text { Bin } \\
\text { mir } \\
\text { un- } \\
\text { sicher }\end{array}$ & Nein & $\begin{array}{c}\text { Nein, } \\
\text { auf } \\
\text { keinen } \\
\text { Fall }\end{array}$ & $\begin{array}{c}\text { Mittel- } \\
\text { wert }\end{array}$ & $\begin{array}{c}\text { Stand- } \\
\text { ardab- } \\
\text { weich- } \\
\text { ung }\end{array}$ \\
\hline $\begin{array}{l}\text { Die Bilder auf der Verpa- } \\
\text { ckung passen zu den ange- } \\
\text { gebenen Zutaten. }\end{array}$ & $\mathbf{5 , 2} \%$ & $\mathbf{3 7 , 6} \%$ & $\mathbf{1 4 , 4} \%$ & $\mathbf{2 6 , 0} \%$ & $\mathbf{1 6 , 8} \%$ & $\mathbf{- 0 , 1 2}$ & $\mathbf{1 , 2 2 7}$ \\
\hline $\begin{array}{l}\text { Die Produktbezeichnung } \\
\text { „Beeren-Fruchtschnitte“ } \\
\text { passt zu den angegebenen } \\
\text { Zutaten. }\end{array}$ & $\mathbf{1 1 , 9 \%}$ & $\mathbf{2 6 , 8} \%$ & $\mathbf{1 8 , 2} \%$ & $\mathbf{2 7 , 8} \%$ & $\mathbf{1 5 , 2} \%$ & $\mathbf{- 0 , 0 7}$ & $\mathbf{1 , 2 7 6}$ \\
\hline
\end{tabular}

Tabelle 3: Verbraucherverständnis Wellness Wasser

\begin{tabular}{|l|c|c|c|c|c|c|c|}
\hline & $\begin{array}{c}\text { Ja, auf } \\
\text { jeden } \\
\text { Fall }\end{array}$ & Ja & $\begin{array}{c}\text { Bin } \\
\text { mir } \\
\text { un- } \\
\text { sicher }\end{array}$ & Nein & $\begin{array}{c}\text { Nein, } \\
\text { auf } \\
\text { keinen } \\
\text { Fall }\end{array}$ & $\begin{array}{c}\text { Mittel- } \\
\text { wert }\end{array}$ & $\begin{array}{c}\text { Stand- } \\
\text { ardab- } \\
\text { weich- } \\
\text { ung }\end{array}$ \\
\hline $\begin{array}{l}\text { Die Bilder auf der Verpa- } \\
\text { ckung passen zu den ange- } \\
\text { gebenen Zutaten. }\end{array}$ & $\mathbf{4 , 4} \%$ & $\mathbf{3 1 , 0} \%$ & $\mathbf{1 5 , 6} \%$ & $\mathbf{3 0 , 0} \%$ & $\mathbf{1 9 , 1} \%$ & $\mathbf{- 0 , 2 8}$ & $\mathbf{1 , 2 1 3}$ \\
\hline $\begin{array}{l}\text { Die Produktbezeichnung } \\
\text {,Wellness Lychee-Guave“ } \\
\text { passt zu den angegebenen } \\
\text { Zutaten. }\end{array}$ & $\mathbf{7 , 5 \%}$ & $\mathbf{2 4 , 7} \%$ & $\mathbf{1 6 , 5} \%$ & $\mathbf{2 9 , 5} \%$ & $\mathbf{2 1 , 8} \%$ & $\mathbf{- 0 , 3 3}$ & $\mathbf{1 , 2 6 8}$ \\
\hline
\end{tabular}


Tabelle 4: Verbraucherverständnis Instant Nudelsuppe

\begin{tabular}{|l|c|c|c|c|c|c|c|}
\hline & $\begin{array}{c}\text { Ja, auf } \\
\text { jeden } \\
\text { Fall }\end{array}$ & Ja & $\begin{array}{c}\text { Bin } \\
\text { mir } \\
\text { un- } \\
\text { sicher }\end{array}$ & Nein & $\begin{array}{c}\text { Nein, } \\
\text { auf } \\
\text { keinen } \\
\text { Fall }\end{array}$ & $\begin{array}{c}\text { Mittel- } \\
\text { wert }\end{array}$ & $\begin{array}{c}\text { Stand- } \\
\text { ardab- } \\
\text { weich- } \\
\text { ung }\end{array}$ \\
\hline $\begin{array}{l}\text { Die Bilder auf der Verpa- } \\
\text { ckung passen zu den ange- } \\
\text { gebenen Zutaten. }\end{array}$ & $\mathbf{3 , 3} \%$ & $\mathbf{3 0 , 2} \%$ & $\mathbf{1 3 , 3} \%$ & $\mathbf{2 6 , 8} \%$ & $\mathbf{2 6 , 3} \%$ & $\mathbf{- 0 , 4 3}$ & $\mathbf{1 , 2 5 6}$ \\
\hline $\begin{array}{l}\text { Die Produktbezeichnung } \\
\text {,Thai-Food Ente“ passt zu } \\
\text { den angegebenen Zutaten. }\end{array}$ & $\mathbf{6 , 1} \%$ & $\mathbf{2 0 , 4} \%$ & $\mathbf{1 9 , 0} \%$ & $\mathbf{2 6 , 9} \%$ & $\mathbf{2 7 , 6} \%$ & $\mathbf{- 0 , 5}$ & $\mathbf{1 , 2 5 5}$ \\
\hline
\end{tabular}

Tabelle 5: Verbraucherverständnis Ziegenkäse

\begin{tabular}{|l|c|c|c|c|c|c|c|}
\hline & $\begin{array}{c}\text { Ja, auf } \\
\text { jeden } \\
\text { Fall }\end{array}$ & $\mathbf{J a}$ & $\begin{array}{c}\text { Bin } \\
\text { mir } \\
\text { un- } \\
\text { sicher }\end{array}$ & Nein & $\begin{array}{c}\text { Nein, } \\
\text { auf } \\
\text { keinen } \\
\text { Fall }\end{array}$ & $\begin{array}{c}\text { Mittel- } \\
\text { wert }\end{array}$ & $\begin{array}{c}\text { Stand- } \\
\text { ardab- } \\
\text { weich- } \\
\text { ung }\end{array}$ \\
\hline $\begin{array}{l}\text { Die Bilder auf der Verpa- } \\
\text { ckung passen zu den ange- } \\
\text { gebenen Zutaten. }\end{array}$ & $\mathbf{1 1 , 9} \%$ & $\mathbf{4 9 , 0} \%$ & $\mathbf{9 , 7} \%$ & $\mathbf{2 0 , 2} \%$ & $\mathbf{9 , 3} \%$ & $\mathbf{0 , 3 4}$ & $\mathbf{1 , 1 9 3}$ \\
\hline $\begin{array}{l}\text { Die Produktbezeichnung } \\
\text { „Frischkäse mit mildem } \\
\text { Ziegenkäse" passt zu den } \\
\text { angegebenen Zutaten. }\end{array}$ & $\mathbf{1 9 , 6} \%$ & $\mathbf{4 2 , 1} \%$ & $\mathbf{1 5 , 7} \%$ & $\mathbf{1 6 , 5} \%$ & $\mathbf{6 , 2} \%$ & $\mathbf{0 , 5 2}$ & $\mathbf{1 , 1 5 8}$ \\
\hline
\end{tabular}

Tabelle 6: Verbraucherverständnis gefüllte Frischnudeln

\begin{tabular}{|l|c|c|c|c|c|c|c|}
\hline & $\begin{array}{c}\text { Ja, auf } \\
\text { jeden } \\
\text { Fall }\end{array}$ & $\mathbf{J a}$ & $\begin{array}{c}\text { Bin } \\
\text { mir } \\
\text { un- } \\
\text { sicher }\end{array}$ & Nein & $\begin{array}{c}\text { Nein, } \\
\text { auf } \\
\text { keinen } \\
\text { Fall }\end{array}$ & $\begin{array}{c}\text { Mittel- } \\
\text { wert }\end{array}$ & $\begin{array}{c}\text { Stand- } \\
\text { ardab- } \\
\text { weich- } \\
\text { ung }\end{array}$ \\
\hline $\begin{array}{l}\text { Die Bilder auf der Verpa- } \\
\text { ckung passen zu den ange- } \\
\text { gebenen Zutaten. }\end{array}$ & $\mathbf{5 , 5 \%}$ & $\mathbf{3 8 , 0} \%$ & $\mathbf{1 9 , 3} \%$ & $\mathbf{2 9 , 0} \%$ & $\mathbf{8 , 2} \%$ & $\mathbf{0 , 0 4}$ & $\mathbf{1 , 1 0 4}$ \\
\hline $\begin{array}{l}\text { Die Produktbezeichnung } \\
\text {,Triangolini mit Steinpil- } \\
\text { zen und Trüffeln“ passt zu } \\
\text { den angegebenen Zutaten. }\end{array}$ & $\mathbf{7 , 9 \%}$ & $\mathbf{3 3 , 9} \%$ & $\mathbf{1 8 , 3} \%$ & $\mathbf{2 9 , 8} \%$ & $\mathbf{1 0 , 1} \%$ & $\mathbf{0 , 0 0}$ & $\mathbf{1 , 1 6 6}$ \\
\hline
\end{tabular}


Tabelle 7: Verbraucherverständnis Cappuccino ungesüßt

\begin{tabular}{|l|c|c|c|c|c|c|c|}
\hline $\begin{array}{l}\text { Ich verstehe unter dem } \\
\text { Aufdruck ,ungesüßt“, } \\
\text { dass... }\end{array}$ & $\begin{array}{l}\text { Ja, auf } \\
\text { jeden } \\
\text { Fall }\end{array}$ & Ja & $\begin{array}{c}\text { Bin } \\
\text { mir } \\
\text { un- } \\
\text { sicher }\end{array}$ & Nein & $\begin{array}{c}\text { Nein, } \\
\text { auf } \\
\text { keinen } \\
\text { Fall }\end{array}$ & $\begin{array}{c}\text { Mittel- } \\
\text { wert }\end{array}$ & $\begin{array}{c}\text { Stand- } \\
\text { ardab- } \\
\text { weich- } \\
\text { ung }\end{array}$ \\
\hline $\begin{array}{l}\text {...dem Produkt kein Zu- } \\
\text { cker zugesetzt ist. }\end{array}$ & $44,9 \%$ & $35,7 \%$ & $13,7 \%$ & $5,1 \%$ & $0,7 \%$ & 1,19 & 0,903 \\
\hline $\begin{array}{l}\text {...das Produkt einen nied- } \\
\text { rigen Gesamtzuckergeh- } \\
\text { alt aufweist. }\end{array}$ & $18,2 \%$ & $36,1 \%$ & $16,3 \%$ & $21,1 \%$ & $8,3 \%$ & 0,35 & 1,230 \\
\hline $\begin{array}{l}\text {...keine süßenden Zutaten } \\
\text { (z. B. Süßstoff) zugesetzt } \\
\text { sind. }\end{array}$ & $37,3 \%$ & $37,5 \%$ & $15,6 \%$ & $8,7 \%$ & $\mathbf{0 , 9} \%$ & 1,02 & $\mathbf{0 , 9 7 9}$ \\
\hline
\end{tabular}

Tabelle 8: Verbraucherverständnis Erdbeerjoghurt ohne Farbstoffe

\begin{tabular}{|l|c|c|c|c|c|c|c|}
\hline $\begin{array}{l}\text { Ich verstehe unter dem } \\
\text { Aufdruck ,ohne Farb- } \\
\text { stoffe“, dass... }\end{array}$ & $\begin{array}{l}\text { Ja, auf } \\
\text { jeden } \\
\text { Fall }\end{array}$ & Ja & $\begin{array}{c}\text { Bin } \\
\text { mir } \\
\text { un- } \\
\text { sicher }\end{array}$ & Nein & $\begin{array}{c}\text { Nein, } \\
\text { auf } \\
\text { keinen } \\
\text { Fall }\end{array}$ & $\begin{array}{c}\text { Mittel- } \\
\text { wert }\end{array}$ & $\begin{array}{c}\text { Stand- } \\
\text { ardab- } \\
\text { weich- } \\
\text { ung }\end{array}$ \\
\hline $\begin{array}{l}\text {... dem Erdbeerjoghurt } \\
\text { keine Farbstoffe zuge- } \\
\text { setzt wurden. }\end{array}$ & $42,2 \%$ & $37,9 \%$ & $13,9 \%$ & $5,2 \%$ & $0,8 \%$ & 1,16 & 0,903 \\
\hline $\begin{array}{l}\text {... keine färbenden Le- } \\
\text { bensmittel wie z. B. rote } \\
\text { Beete Saft zur Färbung } \\
\text { eingesetzt wurden. }\end{array}$ & $22,0 \%$ & $35,6 \%$ & $25,0 \%$ & $15,0 \%$ & $2,4 \%$ & 0,6 & 1,063 \\
\hline $\begin{array}{l}\text {... die Farbe nur aus Erd- } \\
\text { beeren stammt. }\end{array}$ & $25,2 \%$ & $35,2 \%$ & $21,9 \%$ & $15,3 \%$ & $2,4 \%$ & 0,65 & 1,088 \\
\hline
\end{tabular}

Tabelle 9: Verbraucherverständnis Label frei von Geschmacksverstärkern

\begin{tabular}{|l|c|c|c|c|c|c|c|}
\hline $\begin{array}{l}\text { Ich verstehe unter dem } \\
\text { Aufdruck ,frei von Ge- } \\
\text { schmacksverstärkern“, } \\
\text { dass das Produkt... }\end{array}$ & $\begin{array}{l}\text { Ja, auf } \\
\text { jeden } \\
\text { Fall }\end{array}$ & Ja & $\begin{array}{c}\text { Bin } \\
\text { mir } \\
\text { un- } \\
\text { sicher }\end{array}$ & Nein & $\begin{array}{c}\text { Nein, } \\
\text { auf } \\
\text { keinen } \\
\text { Fall }\end{array}$ & $\begin{array}{c}\text { Mittel- } \\
\text { wert }\end{array}$ & $\begin{array}{c}\text { Stand- } \\
\text { ardab- } \\
\text { weich- } \\
\text { ung }\end{array}$ \\
\hline $\begin{array}{l}\text {...kein Hefeextrakt en- } \\
\text { thält. }\end{array}$ & $\mathbf{1 8 , 8} \%$ & $\mathbf{3 1 , 1} \%$ & $\mathbf{3 0 , 0} \%$ & $\mathbf{1 7 , 0} \%$ & $\mathbf{3 , 0} \%$ & $\mathbf{0 , 4 6}$ & $\mathbf{1 , 0 7 2}$ \\
\hline ...keine Sojasauce enthält. & $\mathbf{1 7 , 5 \%}$ & $\mathbf{3 1 , 4} \%$ & $\mathbf{3 0 , 0} \%$ & $\mathbf{1 7 , 3} \%$ & $\mathbf{3 , 7} \%$ & $\mathbf{0 , 4 2}$ & $\mathbf{1 , 0 7 9}$ \\
\hline ...keine Aromen enthält. & $\mathbf{2 8 , 8} \%$ & $\mathbf{3 5 , 0} \%$ & $\mathbf{1 8 , 5} \%$ & $\mathbf{1 3 , 4} \%$ & $\mathbf{4 , 3} \%$ & $\mathbf{0 , 7 1}$ & $\mathbf{1 , 1 4 5}$ \\
\hline $\begin{array}{l}\text {...keinen Parmesankäse } \\
\text { enthält. }\end{array}$ & $\mathbf{1 7 , 4} \%$ & $\mathbf{3 1 , 3} \%$ & $\mathbf{2 7 , 8} \%$ & $\mathbf{1 9 , 4} \%$ & $\mathbf{4 , 0} \%$ & $\mathbf{0 , 3 9}$ & $\mathbf{1 , 1 0 3}$ \\
\hline
\end{tabular}


II.2 Verbraucherverständnis der Gestaltung von Lebensmittelverpackungen 


\section{Teil II: Consumer understanding of food packaging}

II.3 Zum Verbraucherverständnis von Alpen- und Weidemilch

Autoren: Ramona Weinrich, Sarah Kühl, Anke Zühlsdorf, Achim Spiller

Angenommen zur Veröffentlichung beim German Journal of Agricultural Economics, mit Erlaubnis des Deutschen Fachverlages 


\title{
Zusammenfassung
}

In den letzten Jahren wurden im Zuge der Produktdifferenzierung zunehmend Produkte auf dem Milchmarkt eingeführt, die Verbrauchern einen Zusatznutzen versprechen. Im vorliegenden Artikel wird das Verbraucherverständnis der Produktbezeichnungen Alpenmilch und Weidemilch auf Basis zweier empirischer Studien analysiert. Beide Produktbezeichnungen sind derzeit rechtlich ungeschützte Begriffe. Daher setzen Hersteller die Kennzeichnungen auf der Verpackung in der Praxis sehr unterschiedlich um. Vor diesem Hintergrund wird empirisch analysiert, was Verbraucher unter Alpen- und Weidemilch verstehen. Gehen sie davon aus, dass Kühe bei Alpenmilch in einem bestimmten geografischen Gebiet gehalten werden? Und bedeutet Weidemilch für sie, dass die Milchkühe Auslauf auf der Weide haben und wenn ja, über welchen Zeitraum? Die Ergebnisse der Fallstudien zeigen auf, dass das Verbraucherverständnis bei Produkten, die mit nicht beobachtbaren Prozesseigenschaften beworben werden, sehr weitreichend ist und so (Ent-) Täuschungspotenzial entstehen kann.

\section{Schlüsselwörter}

Milchwirtschaft; Tierwohl; Verbraucherschutz; Lebensmittelkennzeichnung

\begin{abstract}
In recent years in the course of product differentiation more and more dairy products have been introduced promising additional benefits for consumers. The present article analyses the consumers' understanding of the products alpine milk and pasture-raised milk by means of two empirical studies. Currently, both product names are legally unprotected terms. Therefore, producers and marketers implement the labelling on the food package very different. Against this background it is empirically analysed how consumers understand alpine milk and pasture-raised milk. Do consumers think that cows giving the milk for alpine milk are kept in a certain geographical region? And does pasture-raised milk mean to them that dairy cattle have access to pasture and if yes during which period? The results of the case studies show that consumer expectations with respect to products which are advertised with unobservable process characteristics are very high which could lead to consumer disappointment.
\end{abstract}

\section{Keywords}

Dairy industry; animal welfare; consumer protection; food labelling 


\section{Einleitung}

Im Zuge der Ausdifferenzierung von Lebensmittelmärkten werden zunehmend Produkte angeboten, die Verbrauchern einen Zusatznutzen versprechen. Dies ist insbesondere bei sonst eher homogenen, wettbewerbsintensiven Produkten wie Konsummilch attraktiv. Neben Produktvarianten wie Bio-Milch, GVO-freie Milch oder faire Milch finden sich derzeit auch Alpen- bzw. Weidemilch in den Regalen der Supermärkte. Rechtlich geschützt sind diese Begriffe nicht, es gibt lediglich Vorschriften für die Bezeichnungen Bio bzw. ökologisch hergestellt und GVO-frei. Es ist nicht spezifisch geregelt, welche geographischen Merkmale eine Alpenmilch aufweisen oder wie viele Stunden eine Milchkuh täglich auf der Weide stehen bzw. welches Futter sie aufnehmen sollte. Dementsprechend unterschiedlich sind die Qualitätsanforderungen und Kriterien der Hersteller. Es gibt Hersteller, die keine Spezifikationen nennen, andere schreiben einen Weidegang von mindestens sechs Stunden an 120 Tagen im Jahr vor. Die Haltungsform kann jedoch Einfluss auf die für Verbraucher wichtigen Aspekte wie Tierwohl nehmen (SCHLEYER et al., 2013). Aufgrund der Fütterung können auch die Milchzusammensetzung und damit die (sensorische) Qualität unterschiedlich sein (WEIß et al., 2006).

Bei Erzeugnissen wie Alpen- und Weidemilch stellt sich die Frage, wie Verbraucher die jeweiligen Bezeichnungen verstehen und ob sie ggf. enttäuscht sind, wenn ihre Vorstellungen vom tatsächlichen Produktionsprozess abweichen. Diese Fragen wurden bisher in der Forschung kaum berücksichtigt, sind aber aus verbraucher- und wettbewerbspolitischer Hinsicht sowie unter Nachhaltigkeitsaspekten relevant. Dass es hier Probleme geben könnte, deuten eine Reihe kritischer Medienberichte an (z. B. BRÜHL, 2011; KIRSCHEY und HAUER, 2012; Kuhlmann, 2012). Auch die Verbraucherzentralen bemängeln die Diskrepanz zwischen der werblichen Darstellung und den Produktionsrealitäten bei Molkereiprodukten (VERBRAUCHERZENTRALe HAmburg, 2013a und 2013b; Verbraucherzentrale Hessen, 2013). In der vorliegenden Studie wird deshalb anhand von Fallstudien analysiert, wie weitreichend das Verbraucherverständnis von Produktbezeichnungen bei nicht beobachtbaren Prozesseigenschaften ist. In grundsätzlicher Hinsicht leistet die Analyse damit einen Beitrag zur Qualitätskommunikation in Nischenmärkten mit hoher Informationsasymmetrie. 


\section{Milchkennzeichnung in Deutschland und Europa}

\subsection{Gesetzeslage in Deutschland und dem europäischen Ausland}

In Deutschland ist die Bezeichnung von Milch in der Verordnung über die Kennzeichnung wärmebehandelter Konsummilch (Konsummilch-Kennzeichnungs-Verordnung - MilchKennzV) und durch die Verordnung über Milcherzeugnisse (Milcherzeugnisverordnung MilchErzV) gesetzlich geregelt. Festgelegt wird hier z. B. das Inverkehrbringen von Konsummilch, der Fettgehalt von Buttermilch oder der Fettgehalt von Milchprodukten wie fettarmem Joghurt. Die Verordnungen beziehen sich auf Aspekte der Produktqualität, die am Produkt überprüfbar sind. Schwieriger wird es hingegen, wenn landwirtschaftliche Prozesseigenschaften Einfluss auf die Qualität haben und als Marketingmittel dienen, da Prozesseigenschaften am Produkt selber nicht nachvollzogen werden können. Derzeit gibt es in Deutschland lediglich für die Prozesseigenschaft der GVO-Freiheit (Art. 3 § 3a BGBl. 1 Nr. 12 2008) und für ökologisch erzeugte Milch gesetzliche Regelungen (EG-Öko-Basisverordnung [EG] Nr. 834/2007). Entsprechend finden sich immer wieder Diskussionen um Milchbezeichnungen, in denen Prozesseigenschaften angesprochen werden, die gesetzlich nicht festgelegt sind, denn eben solche gewinnen im Lebensmittelmarketing an Relevanz.

Auch innerhalb der EU gibt es bisher kaum gesetzliche Regelungen, die sich auf die Prozesseigenschaften von Milch und Milchprodukten beziehen. $\mathrm{Zu}$ den wenigen vorhandenen Regelungen gehört die EU-Verordnung Nr. 1151/2012, die u. a. das Ziel verfolgt, schwer zu bewirtschaftende Berggebiete für die Landwirtschaft attraktiver zu machen. Die für eine Kennzeichnung als Bergprodukt zu erfüllenden Kriterien beziehen sich auf die Höhen- und Hanglage des Ortes der landwirtschaftlichen Produktion (EUROPÄISCHE GEMEINSCHAFTEN, 2005: 5). Ebenfalls auf die Herkunftskennzeichnung ausgerichtet sind die Siegel zur geschützten Ursprungskennzeichnung (g. U.) und der geschützten geografischen Angabe (g. g. A.). Vorgaben zur Haltung und Fütterung der Kühe sind in den Produktspezifizierungen eher selten (vgl. im Register DOOR der EU aufgeführte Produkte; EUROPÄISCHE KOMMISSION, o. J.). Eine Ausnahme bildet z. B. die Herstellungsbeschreibung des französischen Käses „Emmental francais est-central“", welcher als g. g. A. gekennzeichnet ist. Hier ist neben der geografischen Herkunft auch festgelegt, dass die Kühe „nur mit Gras und Heu gefüttert“ werden und „mindestens 5 Monate auf der Weide [verbringen], damit der Zusammenhang mit den Wiesen im geografischen Gebiet erhalten wird“ (EUROPÄISCHE KOMMISSION, 2012).

Aufgrund der kaum vorhandenen gesetzlichen Regelungen gibt es in der EU bereits einige privatwirtschaftliche Standards, durch welche die Produktionsweise bzw. der Produktionsort 
für Milchprodukte genauer geregelt ist. Ein Beispiel dafür ist die silagefreie Heumilch aus Österreich. Im österreichischen Heumilchregulativ der ARGE Heumilch Österreich ist festgehalten, dass jegliche Silofuttermittel verboten sind. Zudem müssen bei der Herstellung die Richtlinien des Österreichischen Programms für umweltgerechte Landwirtschaft (ÖPUL) eingehalten werden (ARGE Heumilch ÖsterReICH, 2013), wodurch auch die Anzahl der Tiere auf den Weideflächen geregelt wird. Zusätzlich sind die herstellenden Betriebe zertifiziert und werden regelmäßig kontrolliert (ARGE HEUMILCH ÖSTERREICH, o. J.).

In den Niederlanden findet sich ein Programm zur Erhaltung des Weidegangs bei Milchkühen. Die Stiftung „Stichting Weidegang“, an der neben weiteren Unternehmen und Institutionen auch der niederländische Tierschutzverbund „Dieren bescherming“ und die Molkerei Friesland Campina beteiligt sind (Stichting Weidegang, 2014), hat die Mindestdauer des Weidegangs für Milchkühe auf „120 Tage im Jahr für mindestens sechs Stunden pro Tag“ festgelegt (FRIESLANDCAMPINA, 2013), um die Milch als Weidemilch vermarkten zu dürfen. Angaben zur Zufütterung finden sich nicht. Weiterhin muss der Weidegang auf niederländischen Weiden stattfinden (STICHTING WEIDEGANG, o. J.).

\subsection{Verwendung der Begriffe Alpen- und Weidemilch durch die Hersteller}

Aufgrund der fehlenden Regelungen ist der Umgang der Hersteller in Deutschland mit den Begriffen Alpen- und Weidemilch sehr unterschiedlich. Die für Milka Schokolade der Kraft Foods Deutschland GmbH verwendete Alpenmilch stammt „aus Tälern und Hochtälern des Alpenraums sowie aus den Ausläufern der Alpen“. Wie die Ausläufer der Alpen geografisch eingegrenzt werden, wird nicht angegeben (HEIMES, 2012). Der Schokoladenhersteller Ritter erklärt, die Herkunft der Milch für die Sorte Alpenmilch sei „geheim“ (ebd.). Die Schokolade Alpia der Firma Stollwerck GmbH mit Firmensitz in Köln stammt nach eigener Aussage der Firma fast ausschließlich aus Deutschland (ebd.). Eine weitere Einschränkung findet nicht statt, obwohl die Verbraucher aufgrund des Namens und des grafischen Bezugs auf der Verpackung das Produkt mit der Herkunft aus den Alpen assoziieren könnten. Die Molkerei Weihenstephan will den seit einigen Jahren verwendeten Begriff der Alpenmilch nicht weiter auf Verpackungen aufdrucken, obwohl in insgesamt 14 Prozessen gerichtlich entschieden wurde, dass die Bezeichnung verwendet werden darf. Einerseits wolle man so weiteren Anfeindungen vorgreifen und zudem der geplanten Unternehmensexpansion, die verbunden ist mit der Erweiterung des Rohstoffgebiets, gerecht werden (MERKUR-ONLINE, 2015).

Ähnliches zeigt sich bei der Weidemilch: Schwarzwaldmilch Freiburg bewirbt seine Schwarzwälder Weidemilch damit, dass den Kühen „ein Weidegang von Frühjahr bis Herbst 
sowie eine natürlich Futtergrundlage" zur Verfügung stehen (Echt-SCHWARZWALD, o. J.). Auf der Weidemilchverpackung von Hansano wird angegeben, dass die Kühe, die die Weidemilch geben, von Mai bis Oktober mindestens sechs Stunden täglich grasen, insgesamt an mindestens 120 Tagen im Jahr (HANSANO, 2012). Ob zusätzlich zum Weidegras zugefüttert wird, ist nicht angegeben. Ebenfalls hat Arla Foods Weidemilch im Programm. Arla gibt an, dass die Kühe von Frühjahr bis Herbst an mindestens 120 Tagen für mindestens sechs Stunden täglich auf der Weide sind und frisches Weidegras fressen. Im Winter bestehe das Futter weitgehend aus Weidegras (ARla Foods Deutschland GMBH, o. J.). Die Milchwerke Schwaben vermarkten unter der Marke Weideglück Milchprodukte ohne etwas zum Weidegang der Kühe auszusagen.

Es zeigt sich an diesen Beispielen, dass ein Teil der Hersteller Begriffe wie Alpen oder Weide sehr frei als Werbeclaim nutzt. Andere Hersteller haben eigene Kriterien entwickelt, die sich auf Herkunft, Haltung und / oder Futter beziehen. Für Konsumenten ist zumeist nicht oder nicht auf den ersten Blick ersichtlich, welches Produktversprechen mit den beiden Bezeichnungen verbunden ist.

\subsection{Alpen- und Weidemilch: Werbeclaim oder Vertrauensgut?}

Sowohl der gesundheitliche Nutzen von Milch für die menschliche Ernährung als auch Tierwohl- und Umweltaspekte können wichtige Kaufmotive für Milchprodukte darstellen (HELLBERG-BAHR et al., 2012). Der Gesundheitswert steht u. a. im Zusammenhang mit der Fettsäurezusammensetzung der Milch (WEISS, 2007: 83), welcher wiederum Rückschlüsse auf die Futtergrundlage zulässt. Je mehr Silage die Tiere aufnehmen, desto geringer ist der Anteil ungesättigter Fettsäuren in der Milch. Die Futtergrundlage ist für die Verbraucher selbst nicht überprüfbar, grundsätzlich können im Labor jedoch Rückschlüsse gezogen werden, über welche sich die Verbraucher zum Beispiel bei Ökotest informieren können (ÖKOTEST, 2014). Zudem kann eine Isotopenanalyse Aufschluss über die regionale Herkunft der Milch geben, was für die Alpenmilch von Bedeutung ist (vgl. CAMIN et al., 2004; PELLERANO et al., 2008; ZHAO et al., 2012). Solche Eigenschaften weisen damit Vertrauensgutcharakteristika im Sinne der Informationsökonomie auf: Sie sind für den einzelnen Konsumenten nicht direkt verifizierbar, können aber von externen Institutionen und Medien mit mehr oder weniger großem Aufwand offen gelegt werden (JAHN et al., 2005).

Das Haltungssystem, welches häufig direkten Einfluss auf das Tierwohl hat, gestaltet sich in der Überprüfung noch schwieriger. Auf welche Weise die Tiere gehalten werden, kann nur über ein Zertifizierungssystem überprüft werden. Es handelt sich daher um ein sogenanntes 
„Potemkin-Gut". Potemkin-Eigenschaften können nicht ohne weiteres im Nachhinein von Außenstehenden, weder durch die Verbraucher noch durch eine Testinstitution, überprüft werden, sondern verlangen einen Einblick in den Produktionsprozess (JAHN et al., 2005; TIETZEL und WEBER, 1991). Solche nicht beobachtbaren Prozesseigenschaften finden sich auch in anderen Bereichen der Lebensmittelproduktion wie z. B. bei regionalen bzw. ökologisch erzeugten Lebensmitteln oder Animal Welfare-Produkten.

StOCKEBRAND und SPILLER (2009) haben aufgezeigt, dass Verbraucher je nach Region den Begriff Regionalität unterschiedlich eng definieren. BECKER und BRENNER (2000) zeigten, dass es bei regionalen Angaben von Herstellerseite große Unterschiede in der Auslegung regionaler Herkunftsangaben geben kann. Weiterhin zeigte eine Befragung von Biolebensmitteln-Käufern, dass diese davon ausgehen, dass die Futtermittel von einem deutschen Betrieb kommen. Die Probanden waren allerdings negativ überrascht, dass auch Ökobetriebe Futtermittel importieren (WÄGELI und HAMM, 2013). Aus empirischen Studien ist bekannt, dass Verbraucher von ökologisch erzeugten Lebensmitteln erwarten, dass diese gesünder und umweltfreundlicher sind und eine höhere Lebensmittelsicherheit aufweisen, was gleichzeitig die Hauptmotive für den Kauf von ökologisch erzeugten Produkten sind (HARPER und MAKATOUNI, 2002; HERMANSEN, 2003). Somit werden hier ein sehr weites Begriffsverständnis bzw. eine entsprechend weitgehende Erwartungshaltung deutlich. Es gibt jedoch kaum Studien, die belegen, dass eine ökologische Produktion diese Vorteile mit sich bringt (BOURN und PRESCOTT, 2002; SMith-SPANGLER et al., 2012; WiLliAMS et al., 2010).

Eine weitreichende Erwartungshaltung der Verbraucher an die Begriffe Alpen- und Weidemilch kann ebenfalls vorliegen. Sollte das Verbraucherverständnis dieser Begriffe weit über die eigentlichen Produkt- bzw. Prozesseigenschaften hinausgehen, kann dies langfristig zu Problemen führen, wenn diese Diskrepanz auf Seiten der Verbraucher aufgedeckt wird (BOOGAARD et al., 2010; MACK und RossIER, 2009; WEINRICH et al., 2014).

Ohne die Möglichkeit der Überprüfung oder eine feste Regelung bleibt für Verbraucher ein Interpretationsspielraum, wie sie die Produktbezeichnungen Weidemilch oder Alpenmilch auffassen. Ein einfaches semantisches Verständnis der Bezeichnungen würde bedeuten, dass eine Alpenmilch aus dem benannten geografischen Gebiet kommt. Über Auslauf, Futtergrundlage oder Höhenlage wäre damit noch nichts ausgesagt. Bei einer Weidemilch beinhaltet die semantische Assoziation den Weidegang, wobei die Dauer des Weidegangs oder die Futteraufnahme offen bleiben. Aufgrund fehlender weiterer Forschungsergebnisse wird hieraus keine konkrete Hypothese, sondern folgende Forschungsfrage abgeleitet: Wie verstehen Ver- 
braucher die Begriffe Alpen- und Weidemilch? Konkret sollen anhand von empirischen Studien folgende Fragen beantwortet werden: Wie weitreichend ist die Verbrauchererwartung bei beiden Begriffen? Gehen die Verbraucher von einem eher engen Begriffsverständnis aus oder haben sie auch konkrete Erwartungen an die Futtergrundlage, die Dauer des Weidegangs oder die geografische Lage der Betriebe? Generell liefern die Ergebnisse damit einen wichtigen Beitrag, um zu erfassen, wie weitreichend das Begriffsverständnis von nicht beobachtbaren Prozesseigenschaften bei Verbrauchern ausgeprägt ist.

\section{Wahrnehmung durch die Verbraucher - Empirische Erhebungen}

Im Folgenden soll empirisch geklärt werden, wie deutsche Verbraucher die Begriffe Alpenmilch und Weidemilch verstehen. Es handelt sich um zwei verschiedene Studien, wobei die Stichproben aufgrund gesetzter Quoten jeweils hinsichtlich Alter, Geschlecht, Bildung und der regionalen Verteilung, für die erste Studie zusätzlich für Einkommen, ungefähr der Verteilung in der deutschen Bevölkerung entsprechen für die deutsche Bevölkerung. Daher können die Ergebnisse auf die Grundgesamtheit der deutschen Konsumenten übertragen werden. Die genauen Ergebnisse der soziodemografischen Daten sind den einzelnen Studien in 3.1 und $3.2 \mathrm{zu}$ entnehmen. Vor beiden Feldphasen wurden Pretests mit $\mathrm{n}=10$ durchgeführt, um die Verständlichkeit der Formulierungen für die Probanden sicherzustellen. Dabei wurde die Methode des Lauten Denkens verwendet, indem die Probanden ihre Gedanken während des Ausfüllens des Fragebogens verbalisiert haben (ERICSSON und SIMON, 1980).

Zunächst wird die Studie zu dem Begriff Alpenmilch vorgestellt, daran anschließend soll das Verbraucherverständnis des Begriffes Weidemilch analysiert werden.

\subsection{Studie 1: Alpenmilch}

Im Rahmen dieser Studie wurde im Jahr 2012 untersucht, wie Lebensmittelkennzeichnungen und Produktaufmachungen von Verbrauchern verstanden werden. Die Befragung wurde im Auftrag der Verbraucherzentrale Bundesverband e.V. im Rahmen des Projektes Lebensmittelklarheit durchgeführt, welches wiederum durch die Initiative „Klarheit und Wahrheit bei der Kennzeichnung und Aufmachung von Lebensmitteln“ vom Bundesministerium für Ernährung und Landwirtschaft gefördert wird (BMEL, 2014a).

Die Verbraucherbefragung wurde als standardisierte computergestützte persönliche Befragung (CAP-Interviews) durchgeführt. Die Studienteilnehmer waren deutsche Verbraucherinnen und Verbraucher ab 16 Jahren. Die Stichprobengröße beträgt $n=1.021$. 
Das Durchschnittsalter in der Stichprobe liegt bei 43 Jahren. 50,4\% der Teilnehmer sind männlich, dementsprechend sind 49,6\% weiblich. 43,5\% der Haushalte verfügen über ein Nettoeinkommen von bis zu $2.500 € .21 \%$ der Probanden leben in Ostdeutschland, 27,9\% in Süddeutschland, $35,5 \%$ in der Mitte und $15,6 \%$ sind in Norddeutschland wohnhaft. Über einen Hochschulabschluss oder (Fach-) Abitur verfügen 27,1 \% der Probanden. 99,1 \% kaufen „sehr häufig“, „häufig“ oder „manchmal“ Lebensmittel ein. Die ausführlichen soziodemografischen Daten und eine Gegenüberstellung der Ergebnisse und der amtlichen Statistik sind Anhang 1 zu entnehmen.

Das Fallbeispiel Alpenmilch greift den Ortsbezug zu einem natürlichen geografischen Gebiet auf. Hierbei soll untersucht werden, was Verbraucher unter einer solchen Produktbezeichnung verstehen und inwieweit sie weitere Prozessmerkmale mit der Herkunft verbinden. Zur Untersuchung des Herkunftsverständnisses wurde die Stichprobe in zwei Splits aufgeteilt. Die oben erwähnten Quoten wurden ebenfalls innerhalb der Splits gesetzt. 512 Probanden wurden gefragt, wo ihrer Meinung nach ein Produkt mit der Bezeichnung Alpenmilch herkommt, 509 Teilnehmer wurden gefragt, wo eine solche Milch ihrer Meinung nach herkommen sollte. Durch die unterschiedliche Wortwahl in den Splits wird zum einen untersucht, wo Alpenmilch geografisch verortet wird (,herkommt“), zum anderen wird die normative Vorstellung der Konsumenten erfasst, d. h. wo eine Alpenmilch „herkommen sollte“.

Der Unterschied zwischen beiden Fragen könnte Hinweise darauf zulassen, ob ein Misstrauen gegenüber den Herstellerangaben besteht. Ist die Antwortverteilung in beiden gleichverteilt, so würde dies bedeuten, dass die Produktbezeichnung von Verbrauchern nicht angezweifelt wird, was den Herkunftsort der Milch angeht. Als Antwortkategorien waren Deutschland, Süddeutschland, Bayern, Alpen und Alpenvorland und Alpen vorgegeben ${ }^{11}$. Tabelle 1 gibt die Verteilung der Antworten für den ersten Split wieder.

Tabelle 1. Herkunftsverständnis Alpenmilch, $\mathrm{n}=509$

\begin{tabular}{|c|c|c|c|c|}
\hline Aus Deutschland & Aus Süddeutschland & Aus Bayern & $\begin{array}{c}\text { Aus den Alpen und } \\
\text { dem Alpenvorland }\end{array}$ & Aus den Alpen \\
\hline $9,8 \%$ & $12 \%$ & $16,1 \%$ & $36,1 \%$ & $25,9 \%$ \\
\hline
\end{tabular}

Woher kommt Ihrem Verständnis nach eine deutsche Milch mit der Bezeichnung Alpenmilch?

\footnotetext{
11 Die Antwortskala ist als Ordinalskala konstruiert worden. Aus Gründen der Eindeutigkeit wurde im Fragetext nach einer deutschen Milch gefragt. Die Antwortkategorien wurden so programmiert, dass die Probanden die Kategorien nebeneinander - als Reihenfolge ersichtlich - vor Augen hatten.
} 
Insgesamt sind mit $62 \%$ mehr als die Hälfte der Teilnehmer der Auffassung, dass eine Alpenmilch aus den Alpen oder den Alpen und dem Alpenvorland kommt. Die zweite Hälfte der Teilnehmer wurde gefragt, wo eine Alpenmilch herkommen sollte. Hier sind 69,2 \% der Teilnehmer der Auffassung, dass eine Alpenmilch aus den Alpen oder aus den Alpen und dem Alpenvorland kommen sollte. Die genaue Prozentverteilung ist Tabelle 2 zu entnehmen. Bei der prozentualen Verteilung der Antworten beider Fragen lässt sich ein Unterschied feststellen, der auf dem $5 \%$ Niveau signifikant ist ( $\mathrm{t}-$ Test; $\mathrm{t}$-Wert $=-2,127 ; \mathrm{p}=0,034)$.

Tabelle 2. Herkunftsverständnis Alpenmilch, $\mathrm{n}=512$

\begin{tabular}{|c|c|c|c|c|}
\hline Aus Deutschland & Aus Süddeutschland & Aus Bayern & $\begin{array}{c}\text { Aus den Alpen und } \\
\text { dem Alpenvorland }\end{array}$ & Aus den Alpen \\
\hline $7,4 \%$ & $11,1 \%$ & $12,3 \%$ & $39,5 \%$ & $29,7 \%$ \\
\hline
\end{tabular}

Woher sollte Ihrem Verständnis nach eine deutsche Milch mit der Bezeichnung Alpenmilch kommen?

Tabelle 3 zeigt, wie weitreichend Verbraucher den Begriff Alpenmilch interpretieren und nicht allein auf die Herkunft der Milch beziehen. Der Großteil (56,4 \%) verbindet mit der Bezeichnung, dass die Kühe regelmäßig auf die Weide kommen. Ebenso geht über die Hälfte $(66,4 \%)$ davon aus, dass auch die verarbeitende Molkerei ihren Sitz im Alpenraum hat. Lediglich $27,7 \%$ vermuten, dass es sich bei der Produktbezeichnung um eine reine Werbeaussage handelt.

Tabelle 3. Produktionsverständnis Alpenmilch, $\mathrm{n}=1.021$, Angaben der Statements in Prozent, randomisiert

\begin{tabular}{|c|c|c|c|c|c|c|}
\hline $\begin{array}{c}\text { Ich verstehe unter der Bezeich- } \\
\text { nung „Alpenmilch“, dass... }\end{array}$ & $\begin{array}{c}\text { Ja, auf } \\
\text { jeden Fall }\end{array}$ & Ja & $\begin{array}{c}\text { Bin mir } \\
\text { unsicher }\end{array}$ & Nein & $\begin{array}{c}\text { Nein, auf } \\
\text { keinen Fall }\end{array}$ & $\begin{array}{c}\text { MW } \\
\text { (Stan- } \\
\text { dardab- } \\
\text { weichung) }\end{array}$ \\
\hline $\begin{array}{c}\text { f..die Molkerei, die die Milch ver- } \\
\text { arbeitet, ihren Sitz im Alpenraum } \\
\text { hat. }\end{array}$ & 18,3 & 48,1 & 17,6 & 13,3 & 2,6 & 0,18 \\
$(1,008)$
\end{tabular}

Systematische Zusammenhänge zu soziodemografischen Merkmalen zeigten sich nicht $\left(\mathrm{Chi}^{2}\right.$ Tests / Kreuztabellen). Demnach sind die Ergebnisse unabhängig von Geschlecht, Alter, Einkommen und Bildung gültig. 


\subsection{Studie 2: Weidemilch}

Die Datenerhebung der zweiten Studie fand im Juli 2013 statt und wurde im Auftrag eines Molkereiunternehmens durchgeführt. Als Methode zur Rekrutierung der Probanden wurde ein Online Access Panel gewählt. Die Stichprobengröße beträgt 1.009 Probanden.

Der Altersdurchschnitt liegt bei 41 Jahren. 49,4 \% der Teilnehmer sind männlich, 50,6 \% sind weiblich. 20,7\% leben in Ostdeutschland, 27,5\% im Süden, 36,1\% in der Mitte Deutschlands und 15,8 \% der Probanden sind in Norddeutschland wohnhaft. 26,4 \% haben das (Fach-) Abitur oder einen Hochschulabschluss erworben. 26,5\% leben alleine, 35,6 \% in einem zwei-Personen-Haushalt. Bei der Frage nach dem Haushaltsnettoeinkommen haben 17,9 \% keine Angabe gemacht. 57,9\% der Haushalte haben ein Nettoeinkommen von weniger als $2.500 €$ zur Verfügung, 24,0\% stehen $2.500 €$ oder mehr zur Verfügung. 17,9\% haben hier keine Angabe gemacht. 96,5 \% kaufen „sehr häufig“, „häufig“ oder „manchmal“ Lebensmittel ein. Die ausführlichen Ergebnisse der soziodemografischen Daten sind Anhang 2 zu entnehmen.

Tabelle 4 zeigt die Zustimmung von Verbrauchern zu verschiedenen Weidehaltungssystemen. Zuvor erhielten die Konsumenten einen Einleitungstext, der sie darüber aufklärte, dass die Handhabung der Landwirte bezüglich der Dauer des Weidegangs und der Zufütterung unterschiedlich ist (Befragungstext: Bei der Weidehaltung von Milchkühen gibt es unterschiedliche Formen. So wird es z. B. unterschiedlich gehandhabt, wie lange die Kühe draußen auf der Weide sind oder ob sie noch zusätzliches Futter erhalten. Die traditionelle Weidehaltung bedeutet, dass die Kühe im Sommer Tag und Nacht auf der Weide stehen und nur Gras zu fressen bekommen. Das können heute nicht mehr alle Landwirte so umsetzen. Was denken Sie: Sollte ein Produkt auch als Weidemilch bezeichnet werden dürfen, wenn dafür folgende Kriterien gelten?). Die Reihenfolge der Statements (Tab. 4) erfolgte in der Befragung randomisiert. 
Tabelle 4. Verbraucherauffassung Weidemilch, $\mathrm{n}=1.001$, Angaben der Statements in Prozent

\begin{tabular}{|c|c|c|c|c|c|c|}
\hline $\begin{array}{l}\text { Diese Haltungsform sollte als Wei- } \\
\text { demilch bezeichnet werden dürfen. }\end{array}$ & $\begin{array}{l}\text { Stimme } \\
\text { voll und } \\
\text { ganz zu }\end{array}$ & Stimme zu & $\begin{array}{l}\text { Bin mir } \\
\text { unsicher }\end{array}$ & $\begin{array}{l}\text { Stimme } \\
\text { nicht zu }\end{array}$ & $\begin{array}{l}\text { Stimme } \\
\text { über- } \\
\text { haupt } \\
\text { nicht zu }\end{array}$ & $\begin{array}{c}\text { MW } \\
\text { (Stan- } \\
\text { dardab- } \\
\text { weich- } \\
\text { ung) } \\
\end{array}$ \\
\hline $\begin{array}{l}\text { Die Kühe sind im Sommer Tag und } \\
\text { Nacht auf der Weide. Ihr Futter besteht } \\
\text { ausschließlich aus Weidegras. }\end{array}$ & 56,4 & 24,4 & 15,3 & 3,0 & 0,9 & $\begin{array}{c}1,32 \\
(0,902)\end{array}$ \\
\hline $\begin{array}{l}\text { Die Tiere sind im Sommer tagsüber } 12 \\
\text { Stunden auf der Weide. Ihr Futter be- } \\
\text { steht hauptsächlich aus Weidegras. } \\
\text { Ergänzend wird im Stall zugefüttert. }\end{array}$ & 21,7 & 49,6 & 22,3 & 5,1 & 1,4 & $\begin{array}{c}0,85 \\
(0,865)\end{array}$ \\
\hline $\begin{array}{l}\text { Die Kühe sind im Sommer täglich } 6 \\
\text { Stunden auf der Weide und fressen } \\
\text { dann Weidegras. Ihre Hauptfütterung } \\
\text { findet im Stall statt. }\end{array}$ & 5,8 & 22,2 & 41,3 & 25,8 & 5,0 & $\begin{array}{c}-0,02 \\
(0,955)\end{array}$ \\
\hline $\begin{array}{l}\text { Die Kühe sind im Sommer einmal am } \\
\text { Tag für 1-2 Stunden zum Auslauf auf der } \\
\text { Weide. Die Fütterung findet ausschließ- } \\
\text { lich im Stall statt. }\end{array}$ & 1,7 & 6,6 & 20,6 & 41,6 & 29,6 & $\begin{array}{c}-0,91 \\
(0,954)\end{array}$ \\
\hline $\begin{array}{l}\text { Die Kühe sind auch im Sommer ganztä- } \\
\text { gig im Stall. Ihr Hauptfutter besteht aus } \\
\text { Heu (abgemähtem Weidegras). }\end{array}$ & 2,4 & 6,2 & 17,5 & 21,8 & 52,1 & $\begin{array}{c}-1,15 \\
(1,067)\end{array}$ \\
\hline
\end{tabular}

Anmerkung: Die Mittelwerte wurden berechnet auf einer Skala von -2 (Stimme voll und ganz zu) bis +2 (Stimme überhaupt nicht zu)

Die Ergebnisse zeigen, dass die Zustimmung zur Deklarierung als Weidemilch abnimmt, je weniger Zeit die Tiere auf der Weide verbringen und je weniger Weidegras sie aufnehmen. Stimmen der Deklaration der ersten Haltungsform (ganztägige Weidehaltung im Sommer, Futter besteht nur aus Weidegras) noch $80,8 \%$ zu oder vollkommen zu, so sind es in der letzten Haltungsvariante (ganzjährige Stallhaltung) mit Heufütterung nur noch 8,6\%. Verbraucher, die der engsten Begriffsauffassung nicht zustimmen, antworten größtenteils mit „Bin mir unsicher“. Verursacht kann das dadurch sein, dass über Management und Haltungssysteme wenig Wissen besteht.

In einer zweiten Fragestellung ging es um die Akzeptanz einer Weidemilch mit einem geringen Grünfutteranteil. Die Probanden wurden darauf hingewiesen, dass häufig auch bei Weidehaltung eine $\mathrm{Zu}$ fütterung erfolgt und die Milchqualität je nach Futtergrundlage variiert. Anschließend wurde ihre $\mathrm{Zu}-$ stimmung zu je einem Pro- und einem Contra-Statement abgefragt. (Befragungstext: Kühe, die im Sommer auf der Weide stehen, bekommen neben Weidegras häufig zusätzliches Futter im Stall. Wenn der Grasanteil besonders groß ist, enthält die Milch mehr Omega-3-Fettsäuren als herkömmliche Milch. Nachfolgendend sehen Sie Pro- und Contra-Argumente, ob eine Milch von Kühen mit garantiertem täglichem Weidegang, die aber nur wenig Gras und dafür mehr herkömmliches Futter bekommen, als Weidemilch bezeichnet werden sollte. Wie stehen sie dazu? Statements vgl. Tabelle 5).

Es lässt sich in Tabelle 5 erkennen, dass ein Großteil der Verbraucher $(67,1 \%)$ die Bezeichnung auch bei einer Zufütterung im Stall befürwortet, wenn die Kühe den Tag auf der Weide 
verbringen. Nur 9,1\% finden diese Kennzeichnung falsch oder vollkommen falsch. Gleichzeitig fühlen sich 42,5 \% durch die Bezeichnung getäuscht, weil die Tiere ihren Energiebedarf nicht nur ausschließlich über Grünfutter auf der Weide decken. Der kleinere Teil der Probanden $(18,7 \%)$ fühlt sich durch die Bezeichnung nicht getäuscht. Die Überschneidungen im Antwortverhalten deuten auf die Verunsicherung der Probanden hin. Besonders ausgeprägt ist die Verunsicherung bei dem zweiten Statement in Tabelle 5. 38,8 \% können das Argument nicht einordnen. Da die Reihenfolge der Statements randomisiert war, dürften sich in dem teilweise widersprüchlichen Antwortverhalten widerspiegeln, dass die Einstellung zur Zufütterung bei Weidehaltung bisher wenig verfestigt und das Antwortverhalten überwiegend intuitiv erfolgt ist.

Tabelle 5. Verbraucherzustimmung zur Deklaration Weidemilch, $\mathrm{n}=1.000$, Angaben in Prozent

\begin{tabular}{|l|c|c|c|c|c|c|}
\hline \multicolumn{1}{|c|}{ Statement } & $\begin{array}{c}\text { Finde ich } \\
\text { vollkom- } \\
\text { men } \\
\text { richtig }\end{array}$ & $\begin{array}{c}\text { Finde ich } \\
\text { richtig }\end{array}$ & $\begin{array}{c}\text { Weiß } \\
\text { nicht }\end{array}$ & $\begin{array}{c}\text { Finde ich } \\
\text { falsch }\end{array}$ & $\begin{array}{c}\text { Finde ich } \\
\text { vollkom- } \\
\text { men } \\
\text { falsch }\end{array}$ & $\begin{array}{c}\text { MW } \\
\text { (Stan- } \\
\text { dardab- } \\
\text { weich- } \\
\text { ung) }\end{array}$ \\
\hline $\begin{array}{l}\text { Die Milch sollte Weidemilch heißen, } \\
\text { weil die Kühe ja den ganzen Tag Auslauf } \\
\text { auf der Weide haben. }\end{array}$ & 30,0 & 37,1 & 23,8 & 8,0 & 1,1 & 0,87 \\
\hline $\begin{array}{l}\text { Ich fühle mich durch die Bezeichnung } \\
\text { „Weidemilch“ getäuscht, weil die Tiere } \\
\text { nicht nur auf der Weide fressen. }\end{array}$ & 11,8 & 30,7 & 38,8 & 16,7 & 2,0 & 0,34 \\
\hline
\end{tabular}

Auch in der zweiten Studie konnten keine signifikanten Zusammenhänge zur Soziodemografie (Alter, Geschlecht, Einkommen, Bildung) aufgedeckt werden (Chi ${ }^{2}$-Tests / Kreuztabellen).

\section{Diskussion}

Für die Begriffe Alpen- und Weidemilch gibt es derzeit keine rechtliche Regelung. Bisher ist in keiner anderen Studie das Verständnis der beiden Bezeichnungen untersucht worden. Es konnte an den Umsetzungsbeispielen gezeigt werden, dass die Hersteller sehr unterschiedlich mit den Begriffen umgehen und dies wiederum oftmals nicht mit den anspruchsvollen Vorstellungen der Konsumenten konform geht.

Bei einem einfachen, semantischen Begriffsverständnis würde der Begriff Alpenmilch für eine Milch aus den Alpen stehen und eine Weidemilch würde von Kühen erzeugt werden, die Weidegang haben. Die Ergebnisse der Befragungen haben gezeigt, dass die Verbraucher diese Eigenschaften auch bei den abgefragten Produkten erwarten: 69,2 \% der Probanden gaben an, dass die Alpenmilch ihrem Verständnis nach aus den Alpen oder dem Alpenvorland kommen sollte, aber nur $62 \%$ glaubten, dass die Milch mit einer solchen Kennzeichnung auch tatsäch- 
lich von dort stammt. Hier deuten sich skeptische Erwartungshaltungen an, was mit einem generellen Misstrauen in die Agrar- und Ernährungswirtschaft in Verbindung gebracht werden kann (BMEL, 2014b; NESTLÉ, 2012).

Die Erwartungen und Vorstellungen der Verbraucher gehen jedoch weit über die rein semantischen Assoziationen hinaus. Die Ergebnisse der Fallstudie Alpenmilch zeigen, dass die Probanden neben der geografischen Einordnung davon ausgehen, dass die Kühe, die zur Erzeugung der Alpenmilch gehalten werden, auf einer Weide stehen (56,4\%). Dies kann dadurch erklärt werden, dass die Alpen als Naturraum mit besonders naturnaher Erzeugung und somit auch mit einer positiven Haltungsform assoziiert werden. Dies könnte dadurch erklärt werden, dass Verbraucher Milchviehhaltung generell mit Weidehaltung verbinden und sie auch in diesem Fall erwarten (SPILler, 2014; VAN DEN Pol-VAn DASSElaAR et al., 2002). Weiterhin können auch die Werbemaßnahmen der Hersteller ursächlich sein: Der Begriff Alpenmilch wird oft mit Bildern einer grasenden Kuh in den Bergen untermauert. Aber gerade in der Alpenregion stehen im Winter und teilweise auch im Sommer viele Tiere in Anbindehaltung im Stall, was diesem romantisierenden Bild widerspricht. In Bayern bspw. halten $65 \%$ der konventionellen Betriebe und $40 \%$ der ökologisch wirtschaftenden Betriebe ihre Milchkühe im Winter in Anbindehaltung (SPRENGEL, 2009). In der tierethologischen Forschung gilt Anbindehaltung als zentraler Risikofaktor für das Tierwohl (EFSA, 2009). Es kann davon ausgegangen werden, dass diese Tatsache den meisten Konsumenten nicht bekannt ist.

Auch bezüglich der Weidemilch wird deutlich, dass die meisten Verbraucher nicht nur Weidehaltung erwarten, sondern auch Anforderungen an die Dauer des Weidegangs und die Zufütterung stellen. Die Zustimmung zur Deklarierung einer Milch als Weidemilch nahm bei den Probanden mit zunehmender Dauer des Weidegangs pro Tag zu. Die Ergebnisse zeigen eine Bevorzugung der Vollweide, was heute für wenige Landwirte umsetzbar sein dürfte, da dies bedeutet, dass die Milchkühe im Sommer ganztägig auf der Weide stehen und ihren Energiebedarf ausschließlich über Weidegras decken. Es verfügen nur wenige Betriebe über genügend Flächen, die direkt an den Betrieb angrenzen, um dem gesamten Tierbestand eine Vollweide zur Verfügung zu stellen. Zudem nimmt mit zunehmender Herdengröße die Praktikabilität der Weidehaltung, aber insbesondere einer Vollweide, ab (SCHLEYER et al., 2013). Auch müssen weitere wichtige Aspekte wie Grundfutterleistungen, Genetik, Management oder die Arbeitszeit berücksichtigt werden: So gibt eine Milchkuh bei einer reinen grünfutterbasierten Ernährung weniger Milch als bei einer kraftfutterunterstützen Fütterung (ebd.). Die genetische Disposition beeinflusst wesentlich das Immunsystem und damit, wie resistent eine Kuh gegenüber Umwelteinflüssen ist. Management (Know-how des Tierhalters, z. B. über die 
Bedingungen, die für eine Weidehaltung essenziell sind) und Zeit (z. B. um die Kühe auf die Weide zu treiben bzw. wieder rein zu holen), die ein Tierhalter zur Verfügung hat, beeinflussen ebenfalls den Erfolg und die Praktikabilität einer Weidehaltung. Verbraucher verfügen i. d. R. nicht über das soeben beschriebene Wissen, was zu einem Problem bei der Kommunikation der verschiedenen Systeme in der Milchviehhaltung werden kann. Auf breiter Basis funktionierende und praktikable Lösungen sind daher unter deutschen Bedingungen eher Teilweidesysteme, wie sie derzeit von Arla (o. J.), Friesland CAMPInA (2013) und HansaNO (2012) festgelegt sind (120 Tage im Jahr für mindestens sechs Stunden pro Tag). De facto bedeutet die Zusicherung von sechs Stunden Weidegang an 120 Tagen pro Jahr auch, dass eine Milchkuh ggf. nur 8,2 \% ihrer Zeit auf der Weide verbringt (GAULY, 2015). Die vorliegenden Ergebnisse zeigen, dass ein solches System nur von einem geringen Anteil der Verbraucher unter dem Label Weidemilch akzeptiert wird (28,0 \%) - die größte Zustimmung erhält das Vollweidesystem (80,8 \%). Ein mehrstufiges Labelsystem kann eine Lösung darstellen, um diese unterschiedlichen Standards transparent zu kommunizieren.

Die Diskrepanz von Verbrauchererwartungen und Produktrealität kann zu einem Teil auch auf den sogenannten Halo-Effekt (vgl. THORNDIKE, 1920) zurückgeführt werden: Konsumenten ordnen einer Produktbezeichnung z. T. weit mehr Eigenschaften zu, als das Produkt tatsächlich erfüllt. MATSCHER und SCHERMER (2009) haben in einer Studie zu Bergprodukten festgestellt, dass nicht in erster Linie die Bergregion, sondern vor allem damit im Zusammenhang stehende Aspekte wie „ohne Zusatzstoffe“, „Regionalität“, „,Tiergerechtheit“ oder auch „Gesundheit" als Kaufargumente genannt werden. Die Konsumenten gehen davon aus, dass Produkte aus Bergregionen diese Qualitätseigenschaften besitzen, auch wenn dafür keine Anhaltspunkte gegeben werden. Ähnliches kann bei Bio-Lebensmitteln beobachtet werden: der Begriff Bio dient Verbrauchern als information chunk und ruft Assoziationen wie einen höheren Gesundheitswert im Vergleich zu konventionellen Lebensmitteln hervor, die diese aber nicht zwangsläufig besitzen (CANAVARI et al., 2009; LOBLEY et al., 2009; TORJUSEN et al., 2004; VON MEYER-HÖFER et al., 2013). Hier zeigt sich ein Spannungsfeld zwischen der reinen Begrifflichkeit und den darüber hinausgehenden Erwartungen der Verbraucher, wie sie oben beschrieben wurden. Etwas überspitzt ausgedrückt: Auf Seiten der Verbraucher existieren teilweise agrarromantische Vorstellungen, die in der heutigen, durch immer größere Betriebe gekennzeichneten Landwirtschaft so nicht immer umsetzbar sind - auch nicht in den spezifischen Qualitätssegmenten, bei denen die Verbraucher sich dies erhoffen. Ähnlich weit gefasste Begriffsverständnisse finden sich auch für die Bezeichnung Regionalität (STOCKEBRAND und SPILLER, 2009; WÄGELI und HAMM, 2013). Sind nur wenige verlässliche Qualitätssignale 
vorhanden, werden diese nicht selten von den Konsumenten überinterpretiert (BECKER und BRENNER, 2000).

Die Diskrepanz zwischen Verbrauchererwartungen und Produktrealität kann zu einem anderen Teil aber auch auf problematische, ggf. sogar irreführende Marketingmaßnahmen der Anbieterseite zurückgeführt werden. Wenn Begriffe wie Alpenmilch von einigen Herstellern als einfache Werbeclaims ohne jeden Bezug zur Herkunft der Milch genutzt werden und den Nachfragern klar wird, dass ihre Erwartungen nicht mit der Realität der Produktion überein stimmen (BOOGAARD et al., 2010; MACK und ROSSIER, 2010; WEINRICH et al., 2014), kann dies die verbreitete Ambivalenz und das teilweise geringe Vertrauen in die Ernährungsindustrie weiter fördern (DIE LEBENSMITTELWIRTSCHAFT, 2015).

Aus ökonomischer Sicht kann mangels geeigneten Bewertungsmaßstabs keine zusammenfassende Aussage darüber getroffen werden, ob die in der Befragung ermittelten Diskrepanzen als gering oder hoch einzuschätzen sind. Aus juristischer Sicht können die im Wettbewerbsrecht von den Gerichten verwendeten Irreführungsquoten herangezogen werden. Irreführungsquoten geben den prozentualen Anteil der Personen innerhalb der angesprochenen Verkehrskreise (der Zielgruppe) an, die durch eine bestimmte Werbeaussage irregeführt werden. In wettbewerbsrechtlichen Verfahren gehen die Gerichte i. d. R. davon aus, dass eine relevante Verbrauchertäuschung vorliegt, wenn ein erheblicher Teil (ca. ein Drittel) der Verbraucher die Aussage falsch versteht (VERGHO, 2015). In unserer Befragung zeigen sich folgende Anteile von Konsumenten, die einem der vorgelegten Standards widersprechen und diese damit falsch verstehen würden: Bei einem Vollweidesystem liegt die Ablehnungsquote nur bei 3,9\%, der Standard entspricht damit den Verbrauchererwartungen sehr weitgehend. Beim derzeit in der Praxis angewandten 120 / 6-Standard beträgt der Anteil der Ablehner 30,8 \%, liegt also im grenzwertigen Bereich. Klar abgelehnt als Weidemilch werden hingegen die Joggingweide (71,2 \%) und die Heufütterung im Stall (73,9\%), solche Systeme würden Verbraucher keiner Weidemilch zuordnen. Da in der Befragung allerdings nicht konkret nach ihrem Verständnis, sondern nach ihrer Bewertung gefragt wurde, sind alle genannten Werte nur erste Hinweise auf eine potenzielle Irreführung; es besteht weiterer Forschungsbedarf.

Aus den Befragungsergebnissen lassen sich daher keine einfachen, direkt umsetzbaren Schlussfolgerungen ziehen. Sie zeigen zunächst einen weitreichenden Erwartungshorizont an bestimmte werblich genutzte Begriffe, deren Hintergründe z. B. in Werbekampagnen, allgemeinen Vorstellungen über Landwirtschaft und Ernährungsindustrie und generellen gesellschaftlichen Werteentwicklungen, wie z. B. Tierschutz, liegen können. Für das Marketing ist 
der weitreichende Erwartungshorizont „Fluch und Segen“ zugleich. Eine positive Begriffsaufladung macht solche Bezeichnungen für die Werbung attraktiv. Sie erhöht das akquisitorische Potenzial solcher Label und erleichtert die für das Lebensmittelmarketing essentielle emotionale Kundenansprache. Zugleich aber - so die Ergebnisse insbesondere der Kundenzufriedenheitsforschung - führen größere Differenzen zwischen Erwartung und wahrgenommener Produktleistung zu Kundenenttäuschung (HomBurG, 2008; HolZING, 2008; ZüHLSDORF und SPILLER, 2013). Bei weitgehenden Fällen kann es sogar dazu kommen, dass sich Kunden getäuscht fühlen.

Die einleitend dargestellten Beispiele verdeutlichen, dass der derzeit ungeregelte Markt, in dem außer den grundsätzlichen Generalklauseln zum Irreführungsschutz im Lebensmittelund Wettbewerbsrecht (Art. 7 Abs. 1 LMIV; § 5 UWG) keine spezifischen Regelungen zu den Bezeichnungen vorliegen, die Gefahr unterschiedlicher und tendenziell niedriger Standards hoch ist. Angesichts der hohen Informationsasymmetrie ist es für einzelne Hersteller u. U. erfolgversprechend, die positiv besetzten Bezeichnungen zu nutzen, ohne weitreichende und kostenerhöhende Kriterien einzuhalten. Im Akerlofschen Sinne (AKERLOF, 1970) drohen damit Prozesse der Negativauslese oder anders ausgedrückt: Nicht erfüllte Erwartungen bieten Potenzial für kritische Medienberichte und schlagen sich in einem geringen Verbrauchervertrauen nieder (BRÜHL, 2011; KIRSCHEY und HAUER, 2012; KUHLMANN, 2012).

Vor diesem Hintergrund ist eine für Hersteller wie Kunden klare Begriffsfestlegung bei solchen Prozesseigenschaften sinnvoll. Diese kann von unterschiedlichen Institutionen ausgehen. Das Beispiel Weidemilch zeigt, dass derzeit bereits einzelne Hersteller Standards für sich festlegen und kommunizieren (siehe z. B. ARLA, o. J., HANSANO, 2012). In den Niederlanden ist über eine Stiftung ein privatwirtschaftlicher Weidemilchstandard für die gesamte Branche erarbeitet worden (STICHTING WEIDEGANG, 2014). Ähnliche Überlegungen gibt es auch für Deutschland beim Milchindustrieverband (mündliche Auskunft). Das Bundesland Niedersachsen hat die Universität Göttingen in einem Forschungsprojekt mit der Fragestellung eines geeigneten Standards betraut (UNIVERSiTÄT GÖTTINGEN, 2014). In anderen, ähnlichen Fallkonstellationen haben sich auch Multi-Stakeholder-Dialoge bewährt (z. B. bei der Entwicklung des MSC-Standards [Marine Stewardship Council] in der Fischwirtschaft, beim Roundtable on Sustainable Palm Oil [RSPO] oder bei Stichting Weidegang). Schließlich zeigt das Beispiel Bergprodukte, dass auch der Gesetzgeber auf nationaler wie internationaler Ebene solche Begriffe schützen kann. Er kann dabei mit dem Instrument der sog. vorbehaltenen Angabe (Begriff darf nur dann verwendet werden, wenn gesetzlich definierte Bedingungen eingehalten werden) Bezeichnungen definieren oder - weitergehend - ein eigenes Label zur 
Kennzeichnung bestimmter Herstellungsprozesse kreieren. Bei einem gesetzlichen Label ist zwischen freiwilliger (z. B. Bio-Siegel) und verpflichtender Kennzeichnung (z. B. Eierkennzeichnung) zu unterscheiden. Letzteres würde eine Vollkennzeichnung aller Milcherzeugnisse mit dem dahinter stehenden Haltungssystem bedeuten.

\section{Fazit}

Es kann vermutet werden, dass Konsumenten von missverständlichen Produktbezeichnungen enttäuscht sind, wenn ihre Erwartungen an ein Produkt nicht erfüllt werden (vgl. auch ZÜHLSDORF und SPILLER, 2012). Bei einer weiterhin ungeregelten Verwendung der Begriffe könnte eine gesamte Branche an Glaubwürdigkeit verlieren. Dann wären auch Kunden mit einer Mehrzahlungsbereitschaft nicht mehr gewillt, für Premiumprodukte einen Aufpreis zu bezahlen. Dies kann langfristig zu Marktversagen führen, wenn Landwirte, die ihren Tieren Weidehaltung ermöglichen bzw. deren Betrieb in der (hinsichtlich der Produktionskosten benachteiligten) Alpenregion liegt, den Mehraufwand finanziell nicht entlohnt bekommen. Langfristig werden sie dann auf andere Produktionsmethoden zurückgreifen, die gewinnbringender sind, oder sie werden die Milchviehhaltung gänzlich aufgeben (AKERLOF, 1970). In diesem Zusammenhang kann auch von einer Misstrauensspirale gesprochen werden, die den Qualitätswettbewerb bedroht (ZÜHLSDORF et al., 2013).

Zusammenfassend kann gesagt werden, dass eine fehlende bzw. intransparente Kommunikation mit den Verbrauchern aufgrund der daraus resultierenden hohen Erwartungen (Ent-)Täuschungspotenzial aufweist. Dies ist besonders kritisch einzuschätzen, da sich bei beiden Studien keine soziodemografischen Zusammenhänge gezeigt haben und somit davon auszugehen ist, dass diese Ergebnisse für die Gesamtbevölkerung gelten. Am Beispiel der Produktbezeichnungen Weidemilch und Alpenmilch konnte gezeigt werden, dass Verbraucher aus Produktbezeichnungen Rückschlüsse auf den Produktionsprozess ziehen. Gehen die Verbraucherassoziationen und Produktionsrealitäten zu weit auseinander, sind Konfliktpotential und Enttäuschung kaum zu vermeiden.

Übertragen auf andere, nicht durch den Konsumenten beobachtbare Prozesseigenschaften sollten Hersteller bei der Verpackungsaufmachung generell darauf achten, dass verbale oder grafische Aufmachungen nicht dazu geeignet sind, unerfüllbare Erwartungen bei Verbrauchern hervorzurufen. Andernfalls können unerfüllte Erwartungen zu Enttäuschung und damit zu Unzufriedenheit führen. Nur eine klare, eindeutige Kommunikation kann langfristig zu Kundenzufriedenheit und damit Kundenbindung führen, was die Etablierung verschiedener Markt- und Nischensegmente auf dem Lebensmittelmarkt ermöglicht. Es wird aber auch deut- 
lich, dass überschießende Erwartungen bei bestimmten Begriffen für die Hersteller nicht einfach zu vermeiden sind. Welche Rolle Wirtschaft, Stakeholder und/oder der Staat bei der notwendigen Definition von Werbebegriffen und dahinter stehenden Prozessstandards einnehmen sollen, kann der vorliegende Beitrag aber nicht beantworten.

Limitierend ist auch anzumerken, dass die Studien methodisch eine erste Annäherung an die Problematik darstellen. Weitere Studien sollten das Verbraucherverständnis von Alpen- und Weidemilch vertiefend aufgreifen. Zurzeit werden bereits mehrere thematisch verwandte Forschungsprojekte durchgeführt, um z. B. Zahlungsbereitschaften oder Verbrauchersegmente differenzierter zu erfassen.

\section{Literatur}

AKERLOF, G. A. (1970): The Market for "Lemons": Qualitative Uncertainty and the Market Mechanism. In: Quarterly Journal of Economics 84 (3):488-500

ARGE (ARBEITSGEMEINSCHAFT) HEUMILCH ÖSTERREICH (2013): Österreichisches Heumilchregulativ. In: http://www.heumilch.at/fileadmin/editors/download/Heumilchregulativ 01_2013_Nov.pdf. Abruf: 02. September 2013.

ARGE (ARBEITSGEMEINSCHAFT) HEUMILCH ÖSTERREICH (o. J.): Liste der akkreditierten Zertifizierungsstellen ARGE Heumilch. In: http://www.heumilch.at /fileadmin/editors/download/Liste_der_akkreditierten_Zertifizierungsstellen_ARGE_Heu milch Mai2009_.pdf. Abruf: 02. September 2013.

ARLA FoOds DeUtSchland GMBH (o. J.): Unser Arla Weidemilchversprechen. In: http://www.arlafoods.de/unsere-marken/marken/arla-weidemilch/arlaweidemilchver sprechen/. Abruf: 13. August 2015.

BECKER, T. und E. BENNER (2000): Zur Problematik der Herkunftsangabe im regionalen Marketing. Stuttgart, Hohenheimer Agrarökonomische Arbeitsberichte Nr. 1. In: https://www.uni-hohenheim.de/fileadmin/einrichtungen/marktlehre/Arbeitsberichte/haanr1.pdf. Abruf: 20. August 2015.

BMEL (BUNDESMINISTERIUM FÜR ERNÄHRUNG UND LANDWIRTSCHAFT) (2014a): Initiative „Klarheit und Wahrheit bei der Kennzeichnung und Aufmachung von Lebensmitteln“. Handlungsbedarf, Ziele und Maßnahmen. (Stand April 2014). In: http://www.bmel.de/SharedDocs/Downloads/Ernaehrung/KlarheitUndWahrheitInitiativeZu sammenstellung.pdf. Abruf: 30. August 2014 .

BMEL (BUNDESMINISTERIUM FÜR ERNÄHRUNG UND LANDWIRTSCHAFT) (2014b): Einkaufsund Ernährungsverhalten in Deutschland. TNS-Emnid-Umfrage des BMEL. In: http://www.bmel.de/SharedDocs/Downloads/Umfragen/TNS-Emnid-EinkaufsErnaehrungs VerhaltenInDeutschland.pdf?_blob=publicationFile. Abruf: 20. August 2014.

BoogaArd, B. K., Bock, B. B., OOsting, S. J., Wiskerke, J. S. C. und A. J. VAN DeR ZiJPP (2010): Social acceptance of dairy farming: the ambivalence between the two faces of modernity. In: Journal of Agricultural and Environmental Ethics 24 (3): 259-282.

Bourn, D. und J. PRESCOTT (2002): A comparison of the nutritional value, sensory qualities, and food safety of organically and conventionally produced foods. In: Critical Reviews in Food Science and Nutrition 42 (1): 1-34. 
BRÜHL, J. (2011): Irreführende Lebensmittelpackungen - Erdbeeren, mit Datteln gestreckt. Süddeutsche.de. Wirtschaft. In: http://www.sueddeutsche.de/wirtschaft/irrefuehrendelebensmittel packungen-erdbeeren-mit-datteln-gestreckt-1.1129249. Abruf: 15. Oktober 2013.

Camin, F., K. Wietzerbin, A. B. Cortes, G. Haberhauer, M. Lees und G. Versini (2004): Application of Multielement Stable Isotope Ratio Analysis to the Characterization of French, Italian, and Spanish Cheeses. In: Journal of Agricultural and Food Chemistry 52 (21): 6592-6601.

Canavari, M., D. Asioli, A. Bendini, N. Cantore, T. Gallina Toschi, A. Spiller, T. OBERMOWE, K. BUCHECKER und M. LOHMANN (2009): Summary report on sensory-related socioeconomic and sensory science literature about organic food products. In: http://orgprints.org/17208/2/ deliverable_1_2_sensory_literature.pdf. Abruf: 02. Februar 2015.

DIE LEBENSMITTELWIRTSCHAFT (2015): Transparenz und Verbraucher - ein Missverständnis? Wesentliche Studienergebnisse im Überblick. In: http://www.neue-verpackung.de/wpcotent/uploads/2014/11/Lebensmittelwirtschaft_2014_11_05_Whitepaper_Transparenzstu die.pdf. Abruf: 01. September 2015.

ECHT-SchwARZWALD (o. J.): Schwarzwälder Weidemilch von Schwarzwaldmilch Freiburg. In: http://www.echt-schwarzwald.de/weidemilch.html. Abruf: 14. August 2013.

EFSA (EUROPEAN FOOD SAFETY AUTHORITY) (2009): Scientific Opinion on the overall effects of farming systems on dairy cow welfare and disease. In: The EFSA Journal (2009) 1143: 8-38, doi: doi:10.2903/j.efsa.2009.1143.

ERICSSON, K. A., und H. A. SIMON (1980): Verbal reports as data. In: Psychological Review 87 (3): $215-251$.

EUROPÄISCHE GEMEINSCHAFTEN (2005): Berggebiete in der Europäischen Union. In: http://ec.europa.eu/agriculture/publi/rurdev/mountain2005_de.pdf. Abruf: 23. Februar 2015.

EUROPÄISCHE KOMMISSION (2012): Veröffentlichung eines Änderungsantrages nach Artikel 6 Absatz 2 der Verordnung (EG) Nr. 510/2006 des Rates zum Schutz von geografischen Angaben und Ursprungsbezeichnungen für Agrarerzeugnisse und Lebensmittel. In: http://eurlex.europa.eu/LexUriServ/LexUriServ.do?uri=OJ:C:2012:352:0017:0021:DE:PDF. Abruf: 04. September 2013.

EUROPÄISCHE KOMMISSION (2013): Geografische Angaben und traditionelle Spezialitäten. In: http://ec.europa.eu/agriculture/quality/schemes/index_de.htm. Abruf: 03. September 2013.

EUROPÄISCHE KOMMISSION (o. J.): Landwirtschaft und Ländliche Entwicklung - DOOR. In: http://ec.europa.eu/agriculture/quality/door/list.html. Abruf: 04. September 2013.

FRIESLANDCAMPINA (2013): Weidegang fördern. In: http://www.friesland campina.com/deutsch/responsibility/outdoor-grazing.aspx. Abruf: 28.10.2013.

Gauly, M. (2015). Was ist besser für das Tierwohl - Weide oder Stall? Vortrag im Rahmen des 7. Rinderworkshops der Deutschen Gesellschaft für Züchtungskunde, Uelzen, 18. Februar 2015.

HANSANO (2012): Hansano Koppelclaim. In: http://www.hansano.de/weidemilch/index2.php. Abruf: 24. Juli 2014.

HARPER, G. C. und A. MAKATOUNI (2002): Consumer perception of organic food production and farm animal welfare. In: British Food Journal 104 (3/4/5): 287 - 299. 
HeIMES, U. (2012): Stammt Alpenmilch von glücklichen Kühen? Beitrag im NDR Fernsehen vom 07.02.2013. In: http://www.ndr.de/ratgeber/verbraucher/lebensmittel/alpen milch101.html. Abruf: 23. Februar 2013.

Hellberg-BAhr, A., N. STEFFen und A. Spiller (2012): Marketingpotentiale für Weidemilch. In: Jahrbuch der Österreichischen Gesellschaft für Agrarökonomie 21 (1): 3-12.

Hermansen, J.E. (2003): Organic livestock production systems and appropriate development in relation to public expectations. In: Livestock Production Science 80 (1-2): 3-15.

Holzing, J. A. (2008): Die Kano-Theorie der Kundenzufriedenheitsmessung. Eine theoretische und empirische Überprüfung. Springer: Wiesbaden.

HomburG, C. (2008) (Hrsg.): Kundenzufriedenheit. Konzepte-Methoden-Erfahrungen. 8. überarbeitete Auflage. Springer: Wiesbaden.

JAhN, G., M. Schramm und A. SPILler (2005): The reliability of certification: Quality labels as a consumer policy tool. In: Journal of Consumer Policy 28 (1): 53-73.

KIRSCHEY, R. und T. HAUER (2012): Traumfabrik Essen - Das Märchen vom natürlichen Lebensmittel. 3 sat. Scobel. In: http://www.3sat.de/page/?source=/scobel/160819/index.html. Abruf: 15. Oktober 2013.

Kuhlmann, S. (2012): Irreführende Bezeichnungen von Lebensmitteln. Deutschlandfunk. Umwelt und Verbraucher. In: http://www.dradio.de/dlf/sendungen/umwelt/1960820/. Abruf: 15. Oktober 2013.

Lobley, M., Butler, A., Courtney, P., Ilbery, B, Kirwan, J. und D. Maye (2009): Analysis of socio-economic aspects of local and national organic farming markets. Final Report for Defra. CRPR Research Report No 29, Centre for Rural Policy Research, University of Exeter.

MACK, G. und R. Rossier (2010): Entspricht die Schweizer Milchproduktion bei erhöhtem Kraftfuttereinsatz noch den Erwartungen der Bevölkerung? In: Loy, J. P. und R. A. E. Müller (Hrsg.): Agrar- und Ernährungsmärkte nach dem Boom, Schriften der Gewisola 45, Landwirtschaftsverlag Münster-Hiltrup, Münster: 353-364.

MAtscher, A. und M. SCHERMER (2009): Zusatznutzen Berg? Argumente für den Konsum von Bergprodukten. In: Agrarwirtschaft 58 (2): 125 - 134.

MERKUR-ONLINE (2015). Keine „Alpenmilch“ mehr aus Freising. In: http://www.merkuronline.de/lokales/freising/freising/keine-alpenmilch-mehr-freising-4666269.html. Abruf: 27. Januar 2015.

Nestlé (2012). Das is(s)t Qualität. Auszüge aus der Nestlé Studie 2012. In: http://www.nestle.de/assetlibrary/documents/verantwortung/nestle\%20studie/executive_summary studie_2012.pdf. Abruf: 20. August 2014.

ÖKOTEST (2014): Milchprodukte - Weide weit weg. In: http://www.oekotest.de /cgi/index.cgi?artnr=102889\&bernr=04. Abruf: 16. Januar 2014.

Pellerano R. G., S. S. Mazza, R. A. Marigliano und E. J. MarchevsKy (2008): Multielement Analysis of Argentinean Lemon Juices by Instrumental Neutronic Activation Analysis and Their Classification According to Geographical Origin. In: Journal of Agricultural and Food Chemistry 56 (13): 5222-5225.

Schleyer, A., W. Lorleberg und M. Mergenthalter (2013): Steigerung der landwirtschaftlichen Wertschöpfung durch Produkte aus Weidehaltungssystemen. Final report 30. 
Fachhochschule Südwestphalen, Soest. In: http://www4.fhswf.de/media/downloads/fbaw_1/download_1/ lorleberg/Weidemilch_Abschlussbericht_131008_final.pdf. Abruf: 17. November 2013.

Smith-Spangler, C., Brandeau, M. L., Hunter, G. E., Bavinger, J. C., Pearson, M., Eschbach, P. J., Sundaram, V., Liu, H., Schirmer, P., Stave, C., Olkin, I. und D. M. BRAVATA (2012): Are Organic Foods Safer or Healthier Than Conventional Alternatives? A Systematic Review. In: Annals of Internal Medicine 157 (5): 348-366.

SPILLER, A. (2014): Gehören Kühe auf die Weide? Ein Denkanstoß zum Image der Haltungssysteme. Universität Göttingen. Diskussionsbeitrag 1402.

SPRENGEL, C. (2009): Anbindehaltung bei Milchkühen in Bayern. Landeskuratorium der Erzeugerringe für tierische Veredelung in Bayern e.V. In: http://www.lkv.bayern.de/media/ Aktuell/Anbindehaltung\%20in\%20Bayern.pdf. Abruf: 08. November 2013.

StATistisches Bundesamt (2012): "Statistisches Jahrbuch. Deutschland und Internationales“, Wiesbaden. In: https://www.destatis.de/DE/Publikationen/StatistischesJahrbuch/ StatistischesJahrbuch2012.pdf? blob=publicationFile. Abruf: 29 Juli 2013.

Stichting WeIDEgANG (2014): Over Stichting Weidegang. In: http://www.stichting weidegang.nl/over-stichting-weidegang.html. Abruf: 16. Januar 2014.

Stichting Weidegang (o. J.): Wat is weidemelk? In: http://www.weidemelk.nl/. Abruf: 13. August 2015.

Stockebrand, N. und A. SpILLER (2009): Regionale Lebensmittel: Sprechen Kunden und Unternehmen die gleiche Sprache? In: Mayer, J.; Alföldi, T.; Leiber, F.; Dubois, D.; Fried, P.; Heckendorn, F.; Hillmann, E.; Klocke, P.; Lüscher, A.; Riedel, S.; Stolze, M.; Strasser, F.; van der Heijden, M. und Willer, H. (Hrsg.) (2009): Werte - Wege - Wirkungen: Biolandbau im Spannungsfeld zwischen Ernährungssicherung, Markt und Klimawandel Beiträge zur 10. Wissenschaftstagung Ökologischer Landbau, ETH Zürich, 11.-13. Februar 2009.

ThORNDIKE, E. L. (1920): A constant error in psychological ratings. In: Journal of Applied Psychology 4 (1): 25-29.

TIETZEL, M. und M. Weber (1991): Von Betrügern, Blendern und Opportunisten. In: Zeitschrift für Wirtschaftspolitik 40: 109-137.

Torjusen, H., Sangstad, L., O'Doherty Jensen, K. und U. KJÆRnes (2004): European Consumers' Conceptions of Organic Food: A Review of Available Research. Report no. 4 - 2004. National Institute for Consumer Research, Oslo.

UNIVERSITÄT GÖTTINGEN (2014): Weideland Niedersachsen - Initiierung eines Weidemilchprogramms zum Schutz des Grünlandes für das Land Niedersachsen. In: http://www.unigoettingen.de/de/499646.html. Abruf: 18. November 2014.

Van den Pol-van Dasselaar, A., C.W. Corré, H. Hopster, G.C.P.M. van LaARhoven und C.W. ROUGOOR (2002): Belang van weidegang. In: http://edepot.wur.nl/34279. Abruf: 17. Februar 2015.

VERBRAUCHERZENTRALE HAMBURG (2013a): Schummelei im Kühlregal. In: http://www.vzhh.de/ernaehrung/255587/schummelei-im-kuehlregal.aspx. Abruf: 23. Februar 2015.

Verbraucherzentrale Hamburg, (2013b): Alles Lug und Trug! In: http://www.vzhh.de/ ernaehrung/306115/alles-lug-und-trug.aspx. Abruf: 23. Februar 2015. 
VerbrauCHERZENTRALE HeSSEN (2013): Wo weidende Kühe drauf sind, muss Weidemilch drin sein! In: http://www.verbraucher.de/wo-weidende-kuehe-drauf-sind--mussweidemilch-drin-sein--1. Abruf: 23. Februar 2015.

VERGHO, R. (2015): Der Maßstab der Verbrauchererwartung im Verbraucherschutzstrafrecht (Studien zum Wirtschaftsstrafrecht). Centaurus Verlag \& Media: Freiburg.

VON Meyer-HöFer, M., S. Nitzko und A. SpILler (2013): Expectation Gaps and HaloEffects in Organic Food Positioning: Characteristics of Organic Food from a Consumer's Point of View. GlobalFood Discussion Papers. No. 25. Universität Göttingen.

WäGELI, S. und U. HAMM (2013): Was heißt hier „regional“"? Verbrauchererwartungen an Öko-Lebensmittel aus tierischer Erzeugung. D. Neuhoff, C. Stumm, S. Ziegler, G. Rahmann, U. Hamm \& U. Köpke (Hrsg.) (2013): Ideal und Wirklichkeit - Perspektiven Ökologischer Landbewirtschaftung. Beiträge zur 12. Wissenschaftstagung Ökologischer Landbau, Bonn, 5. - 8. März 2013, 688-691.

WeInRICH, R., KÜHL, S., SPILlER, A. und A. ZüHLSDORF (2014): Consumer attitudes in Germany towards different dairy housing systems and their implications for the marketing of pasture-raised milk. In: International Food and Agribusiness Management Review (17) 4: 205-222

WEISS, D. (2007): Milch aus Gras - Milch mit Mehrwert für Verbraucher und Bauern? In: Der besondere Wert graslandbasierter Milch, Forschungsanstalt Agroscope LiebefeldPosieux ALP am 8. November 2007: 81 - 85.

Weiß, D., H. Kienberger und H. M. Eichinger (2006): Fettsäuremuster der Milch in Abhängigkeit praxisüblicher Fütterungsstrategien. In: Interdisziplinäres Symposium: Omega 3 Weidemilch - Chancen und Möglichkeiten für Milch- und Rindfleischerzeugnisse vom Grünland. muva Kempten. In: http://www.aktivdrei.de/files/tagungsband_kempten_1.pdf. Abruf: 23. Februar 2013.

Williams, P., Bos, C. und M. Shum (2010): Does Eating Organic Food Reduce Pesticide Exposures and Health Risks? National Collaborating Centre for Environmental Health. In: http://www.ncceh.ca/sites/default/files/Organic_Diet_May_2010.pdf. Abruf: 06. November 2015.

ZhaO H., B. GuO, Y. WeI und B. ZhANG (2012): Effects of Wheat Origin, Genotype, and Their Interaction on Multielement Fingerprints for Geographical Traceability. In: Journal of Agricultural and Food Chemistry 60 (44): 10957-10962.

ZÜHLSDORF, A. und A. SPILLER (2012): Grauzone Lebensmittelkommunikation: Empirische Studie zur Verbraucherwahrnehmung im Spannungsfeld von Informationsanforderungen und Aufmerksamkeitsregeln. Studie im Auftrag der Verbraucherzentralen, Göttingen.

ZüHLSDORF, A., NitZKO, S. und A. SPILlER (2013): Kennzeichnung und Aufmachung von Lebensmitteln aus Sicht der Verbraucher: Empirische Untersuchungsbefunde. Begleitende Verbraucherforschung zum Internetportal www.lebenmittelklarheit.de der Verbraucherzentralen und des Verbraucherzentrale Bundesverbands, Göttingen. 


\section{Anhang 1}

Tabelle 1. Stichprobenbeschreibung - Studie 1

\begin{tabular}{|c|c|c|c|}
\hline Variable & Ausprägung & $\begin{array}{c}\text { Häufigkeiten } \\
\text { (\%) Stich- } \\
\text { probe }\end{array}$ & $\begin{array}{c}\text { Häufigkeiten } \\
(\%) \\
\text { Deutscland }^{12}\end{array}$ \\
\hline Alter & $\begin{array}{l}16 \text { bis } 30 \\
31 \text { bis } 50 \\
\text { Älter als } 50\end{array}$ & $\begin{array}{l}25,0 \\
41,2 \\
33,8\end{array}$ & $\begin{array}{c}24,8 \\
41,2 \\
34\end{array}$ \\
\hline Geschlecht & $\begin{array}{l}\text { Männlich } \\
\text { Weiblich }\end{array}$ & $\begin{array}{l}50,4 \\
49,6\end{array}$ & $\begin{array}{l}49,6 \\
50,4\end{array}$ \\
\hline Region & $\begin{array}{l}\text { Nord } \\
\text { Schleswig-Holstein } \\
\text { Hamburg } \\
\text { Niedersachsen } \\
\text { Bremen } \\
\text { Süd } \\
\text { Baden-Württemberg } \\
\text { Bayern } \\
\text { Ost } \\
\text { Berlin } \\
\text { Brandenburg } \\
\text { Mecklenburg-Vorpommern } \\
\text { Sachsen } \\
\text { Sachsen-Anhalt } \\
\text { Thüringen } \\
\text { West } \\
\text { Nordrhein-Westfalen } \\
\text { Hessen } \\
\text { Rheinland-Pfalz } \\
\text { Saarland }\end{array}$ & $\begin{array}{c}15,6 \\
3,3 \\
2,1 \\
9,4 \\
0,8 \\
27,9 \\
13,2 \\
14,7 \\
21,0 \\
4,2 \\
3,0 \\
2,2 \\
5,4 \\
3,1 \\
3,1 \\
35,5 \\
21,6 \\
7,6 \\
4,7 \\
1,5\end{array}$ & $\begin{array}{c}- \\
3,4 \\
2,1 \\
9,5 \\
0,8 \\
- \\
13,0 \\
15,0 \\
- \\
4,2 \\
3,0 \\
2,1 \\
5,2 \\
3,0 \\
3,0 \\
- \\
21,9 \\
7,4 \\
4,7 \\
1,4\end{array}$ \\
\hline Bildungsabschluss & $\begin{array}{l}\text { Kein Abschluss / noch in der Schulausbildung } \\
\text { Volksschul-/ Hauptschulabschluss } \\
\text { Realschulabschluss / POS } \\
\text { (Fach-) Hochschulreife / (Fach-) Abitur } \\
\text { (Fach-) Hochschulabschluss }\end{array}$ & $\begin{array}{l}7,7 \\
36,3 \\
28,8 \\
13,6 \\
13,5\end{array}$ & $\begin{array}{l}7,8 \\
36,6 \\
28,8 \\
13,6 \\
13,2\end{array}$ \\
\hline $\begin{array}{l}\text { Nettohaushalts- } \\
\text { einkommen }\end{array}$ & Weniger als 1.500 & 15,3 & 15,2 \\
\hline & $\begin{array}{l}1.500-2.500 \\
\text { Mehr als } 2.500 \\
\text { Keine Angabe }\end{array}$ & $\begin{array}{c}28,2 \\
55,4 \\
1,1\end{array}$ & $\begin{array}{c}28,8 \\
55,6 \\
0,4\end{array}$ \\
\hline Haushaltsgröße & $\begin{array}{l}1 \text { Person } \\
2 \text { Personen } \\
3 \text { Personen } \\
4 \text { Personen } \\
\text { Mehr als } 4 \text { Personen }\end{array}$ & $\begin{array}{c}40,3 \\
33,8 \\
12,8 \\
9,6 \\
3,5\end{array}$ & $\begin{array}{l}- \\
- \\
- \\
-\end{array}$ \\
\hline
\end{tabular}

Quelle: eigene Berechnungen; Statistisches Bundesamt (2012); N = 1.021

\footnotetext{
${ }^{12}$ Verteilung in der deutschen Bevölkerung wird nur dort angegeben, wo auch Quoten gesetzt wurden (Alter, Geschlecht, Region, Bildungsabschluss, Nettohaushaltseinkommen); einzelne Summenabweichungen bei den Ausprägungen entstehen durch Ab- und Aufrundungen.
} 


\section{Anhang 2}

Tabelle 1. Stichprobenbeschreibung - Studie 2

\begin{tabular}{|c|c|c|c|}
\hline Variable & Ausprägung & $\begin{array}{c}\text { Häufigkeiten } \\
(\%) \\
\text { Stichprobe }\end{array}$ & $\begin{array}{l}\text { Häufigkeiten } \\
\qquad(\%) \\
\text { Deutscland }^{13}\end{array}$ \\
\hline Alter & $\begin{array}{l}16 \text { bis } 30 \\
31 \text { bis } 50 \\
\text { Älter als } 50\end{array}$ & $\begin{array}{l}26,1 \\
42,8 \\
31,4\end{array}$ & $\begin{array}{c}24,8 \\
41,2 \\
34\end{array}$ \\
\hline Geschlecht & $\begin{array}{l}\text { Männlich } \\
\text { Weiblich }\end{array}$ & $\begin{array}{l}49,4 \\
50,6\end{array}$ & $\begin{array}{l}49,6 \\
50,4\end{array}$ \\
\hline Region & $\begin{array}{l}\text { Nord } \\
\text { Schleswig-Holstein } \\
\text { Hamburg } \\
\text { Niedersachsen } \\
\text { Bremen } \\
\text { Süd } \\
\text { Baden-Württemberg } \\
\text { Bayern } \\
\text { Ost } \\
\text { Berlin } \\
\text { Brandenburg } \\
\text { Mecklenburg-Vorpommern } \\
\text { Sachsen } \\
\text { Sachsen-Anhalt } \\
\text { Thüringen } \\
\text { West } \\
\text { Nordrhein-Westfalen } \\
\text { Hessen } \\
\text { Rheinland-Pfalz } \\
\text { Saarland }\end{array}$ & $\begin{array}{c}15,8 \\
3,6 \\
1,9 \\
9,9 \\
0,4 \\
27,5 \\
12,6 \\
14,9 \\
20,7 \\
4,0 \\
3,2 \\
2,3 \\
5,2 \\
3,2 \\
3,0 \\
36,1 \\
22,0 \\
7,6 \\
5,1 \\
1,4\end{array}$ & $\begin{array}{c}- \\
3,4 \\
2,1 \\
9,5 \\
0,8 \\
- \\
13,0 \\
15,0 \\
- \\
4,2 \\
3,0 \\
2,1 \\
5,2 \\
3,0 \\
3,0 \\
- \\
21,9 \\
7,4 \\
4,7 \\
1,4\end{array}$ \\
\hline Bildungsabschluss & $\begin{array}{l}\text { Kein Abschluss / noch in der Schulausbildung } \\
\text { Volksschul-/ Hauptschulabschluss } \\
\text { Realschulabschluss / POS } \\
\text { (Fach-) Hochschulreife / (Fach-) Abitur } \\
\text { (Fach-) Hochschulabschluss }\end{array}$ & $\begin{array}{c}5,2 \\
40,2 \\
28,2 \\
13,0 \\
13,4\end{array}$ & $\begin{array}{l}7,8 \\
36,6 \\
28,8 \\
13,6 \\
13,2\end{array}$ \\
\hline $\begin{array}{l}\text { Nettohaushalts- } \\
\text { einkommen }\end{array}$ & $\begin{array}{l}\text { Weniger als } 1.500 \\
1.500-2.500 \\
\text { Mehr als } 2.500 \\
\text { Keine Angabe }\end{array}$ & $\begin{array}{l}29,8 \\
28,4 \\
24,0 \\
17,9\end{array}$ & $\begin{array}{l}- \\
- \\
-\end{array}$ \\
\hline Haushaltsgröße & $\begin{array}{l}1 \text { Person } \\
2 \text { Personen } \\
3 \text { Personen } \\
4 \text { Personen } \\
\text { Mehr als } 4 \text { Personen }\end{array}$ & $\begin{array}{c}26,5 \\
35,6 \\
16,8 \\
16,7 \\
4,4\end{array}$ & $\begin{array}{l}- \\
- \\
- \\
-\end{array}$ \\
\hline
\end{tabular}

Quelle: eigene Berechnungen; Statistisches Bundesamt (2012); N = 1.009

\footnotetext{
${ }^{13}$ Verteilung in der deutschen Bevölkerung wird nur dort angegeben, wo auch Quoten gesetzt wurden (Alter, Geschlecht, Region, Bildungsabschluss); einzelne Summenabweichungen bei den Ausprägungen entstehen durch Ab- und Aufrundungen.
} 
II.3 Zum Verbraucherverständnis von Alpen- und Weidemilch 


\section{Teil III: Marketing strategies for niche products}

III.1 Consumer attitudes in Germany towards different dairy housing systems and their implications for the marketing of pasture-raised milk

Autoren: Ramona Weinrich, Sarah Kühl, Anke Zühlsdorf, Achim Spiller

Reprinted with permission from the International Food and Agribusiness Management Review, Volume 17 Issue 4, November, 2014. Copyright held by the International Food and Agribusiness Management Association. 


\section{Introduction}

There is currently much debate surrounding housing systems for dairy cattle. At one end of the spectrum there are the purely indoor housing systems where cows are fed mainly with concentrated feed and that aim to maximize milk production per cow (high-output). At the other end of the spectrum there are cost-minimizing systems that focus on pasture-grazing (low-input) (Steinwidder et al. 2009, Baur et al. 2010). In addition, there are systems that combine free-range and fully housed systems: e.g., housed systems with principally concentrated feed but with access to an outdoor area or systems where cows are at pasture for at least some hours in the summer and have the opportunity of grazing as well as being provided with concentrated feed (Brade 2012). Each system has its advantages and can be cost efficient. This depends on various conditions, such as consolidated pasture, land costs or legal requirements. As a consequence, the focus of the different housing systems differs between countries: e.g., Sweden has regulations for dairy farms that require cows to have access to pasture for at least six hours per day in summer (Spörndly 2012). In countries like Ireland, much grassland is available so outdoor systems with grazing is the obvious choice (Laepple et al. 2012).

Besides some other North-western European countries like the Netherlands, Belgium, or Denmark, Germany is an example for a country with no legal regulations for dairy cow pasturing and an environment which allows different dairy systems. In Germany, the structure of the dairy farms has changed over the last decades. The number of dairy farms has decreased, whereas herd sizes have grown (Federal Statistical Office 2011). Due to the increasing number of cows per farm, the challenges of grazing in Germany are rising. If the farmer wishes to offer free-range for cows, the size of the pastureland has to be proportional to the increased number of cows. But not every farm has the possibility of obtaining more pastureland as the herd size grows (Ostermann-Pfalz and Stöcker 2013). In this case, farmers may opt for a fully housed system where it is possible to enlarge herd size and improve economies of scale. Genetic predisposition also needs to be taken into account as most breeds are bred for high milk performance and so require concentrated feed to achieve the required performance. When they are fully housed it is easier to provide the required feed. Another limiting factor is the workload involved in having to drive the cows to pasture that is far-removed from the farm, as a result of the insufficient number of contiguous parcels (Schleyer et al. 2013). Against these factors, Reijs et al. (2013) predict a decline in German dairy farms with pasturing for cows (regardless of the length of time of pasturing) from almost $50 \%$ in 2012 to less than $5 \%$ in 2025 leading to a new debate in the general public about animal welfare and product quality. 


\section{The Dairy Industry from the Consumer Viewpoint}

The fact that dairy cattle are visible is also important for the public's collective understanding of dairy farming and seems to have a positive influence on its image. At the very least, the image of the dairy industry is not as negative as the image of the meat industry (Schleyer et al. 2013). Several studies show that consumers generally prefer pasturing or at least an access to pasture for animals. Access to open-air is an important criterion for most consumers (Fearne and Lavelle 1996, Deimel et al. 2012). This factor could have an influence on the image of farming systems as well as on the food processing sector. How crucial public perception of a sector is can be seen in the case of battery cages for laying hens. The media debate and corresponding pictures regarding the keeping of laying hens in cages supports the consumer preference for free-range. Pressure from consumers is the reason why eggs from battery hens are now outlawed in Germany (Hörning 2009).

Nevertheless, as shown in the introduction, there is an opposing trend in the German dairy system towards housed dairy cow systems while from a social perspective pasturing should be maintained. On account of these antagonistic requirements, this paper focuses on the German market and consumer perception in Germany. No specific research has been carried out regarding approval of free-range systems (particularly not for the dairy sector). It thus seems very important to obtain deeper insights into consumer attitudes towards the different housing systems so as to avoid situations akin to that of the egg market.

Initial indications that consumers prefer pasture-based systems for dairy cattle is shown by the market share of over $20 \%$ in Denmark for milk which is advertised as pasture-raised milk (Heerwagen et al. 2013). Preliminary efforts are being made to launch pasture-raised milk in Germany. In other countries like the Netherlands (FrieslandCampina), Switzerland (Mirgros), USA (Sweet Meadows Farms) and, as mentioned above, Denmark (Arla Foods), premium products have already been established with the term "pasture" or "meadow" so that higher prices for pasture-raised milk can create an incentive for farmers to continue using pasturebased systems. Moreover, some studies have shown that there is a consumer segment that is willing to pay more for milk from cows that have access to pasture (e.g. Pirog 2004 [USA], Ellis et al. 2009 [UK], Hellberg-Bahr et al. 2012 [GER]). Concern for animal welfare (Ellis et al. 2009) and environmental aspects are identified as major reasons for buying pasture-raised milk, as well as the expectation of a healthier product (Hellberg-Bahr et al. 2012).

In general, quality considerations are important purchasing motivations for some consumers (Caswell and Joseph 2008). The food choice decisions that consumers currently face are al- 
ready very complex and include a wide variety of situational (e.g. time, price), egoistic (e.g. taste, health) and altruistic (e.g. environmental protection, animal welfare) motivations (e.g. Caswell and Joseph 2008, Tsakiridou et al. 2007). In their review Aertsens et al. (2009) describe the personal determinants of organic food consumption. These are actually more abstract values such as "safety", "hedonism", "universalism", "benevolence”, "stimulation", "self-direction" and "conformity". As well as attitude, subjective and personal as well as (perceived) behavioural norms influence the consumption of organic food. Today, milk and milk products are increasingly advertised with additional features for product differentiation. In Germany, the main focus is on GMO freeness, fair payment for dairy farmers, regional origin and the quality attributes of organic milk (Bickel et al. 2009, Zander and Hamm 2010). Environmental and animal welfare characteristics are also important to consumers (Zander and Hamm 2010). McGarry Wolf et al. (2009) showed that the purchasing interest for milk in American organic buyers centered around the qualities of fresh and aromatic taste, safety, high quality, healthy and high nutritional quality, a proper price-quality ratio and a subjectively appropriate price. Many of these aspects can be found in pasture-raised milk. However, pasture-raised milk is based on credence attributes (Akerlof 1970), as consumers must rely on the message that dairy cows have had access to pasture. The marketer therefore needs to provide a sign of reliability.

Although some consumers may show positive attitudes towards pasturing or the furthering of animal welfare issues, a higher price might still be a barrier to purchasing these products (McEachern and Schröder 2002, Padel and Foster 2005, Plaßmann and Hamm 2009). This phenomenon could be explained by the theory of the consumer-citizen-gap. This describes the gap between the attitudes of citizens and their actual behaviour as customers in their shopping situations (especially in respect to animal welfare aspects in food) (Coff et al. 2008, Harvey and Hubbard 2013). While citizens may state that they support pasturing, they may fail to follow through with it in their purchasing as consumers. This has already been shown with the organic food market: Pearson et al. (2011) come to the conclusion that a gap exists between positive attitude and actual behaviour when it comes to making decisions between organic and conventional foods.

This leads to the conclusion that, as well as analysing attitudes toward fully housed and pasturing systems, the survey presented in this paper should integrate the quality orientation of consumers, often related to a high involvement in the product. These consumers are thus more willing to purchase premium products (Aertsens et al. 2009). The assumption of this paper is therefore that there are consumers who prefer pasturing and who are also willing to purchase 
pasture-raised milk, since they are interested in quality. However, at the same time, there are people that, although they may prefer free-range systems as citizens, as consumers they block it out and so do not consider whether the milk originates from pasturing or from fully-housed systems; it is here that the consumer-citizen-gap is present.

In order to integrate the theory of the consumer-citizen-gap found in this literature, this analysis includes the quality aspect. The evidence encourages further research to be undertaken involving the separation of consumers according to their purchasing behaviour for foods, especially milk. This is of particular interest to the German market given the current small market share for pasture-raised products.

\section{Goals}

All the studies presented in the last section focus on whether consumers would purchase pasture-raised milk, on willingness to pay (WTP) analyses or the general preferences of consumers for free-range in livestock farming. The difference in housing systems is not the focal point in this research, or is not even included. What is important is learning more about attitudes towards housing systems and food quality for the purposes of strategic decision-making in the industry concerned.

This paper contributes important background information regarding the perception of housing systems. Due to the issues presented above, regarding decline in pasturing, consumer preference for free-range systems and quality as buying motives, the following three research questions shall be answered:

- How important is pasturing for consumers?

- What is the image of fully housed systems?

- Can the existence of the consumer-citizen-gap regarding quality be verified by different clusters?

It is important for farmers, agricultural technicians and animal shed builders to have this information, so they can be prepared for a possible development regarding consumer expectations. The following factor and cluster analysis have the advantage of combining consumer attitudes towards housed and outdoor systems, while simultaneously separating consumers into different groups depending on their quality orientation. The market potential for pastureraised milk and further manufactured products can thus be established. The results also lead to recommendations for strategic decision-making in the long run. The results are especially important for dairies and the dairy processing industry regarding their long-term business development. 


\section{Methodology}

The data collection took place in July 2013 via an online access panel. The sample size was 1,009. To obtain representative results for the German population, quotas were set for age, gender, education and regional distribution. The survey consisted of questions on milk purchasing frequency as well as the relevance of milk production and milk quality. The focus was on animal welfare aspects of pasture and indoor systems. Respondents scored their answers on a five-point Likert scale. The data was analysed using the statistical software IBM $^{\circledR}$ SPSS, version 21.

First, descriptive analyses showed the impression of consumers when prompted with images of indoor and outdoor systems. The association was measured by a semantic differential. One set of questions showed three images of modern indoor housing systems and another set displayed cows at pasture. Both sets were randomly presented in order to prevent sequence effects. Next, an explorative factor analysis was used to reduce complexity. Finally, a cluster analysis was conducted for the purpose of identifying different consumer clusters. The cluster analysis was performed in several steps to optimize results. Ward's method was used as a cluster method, and the squared Euclidean distance as an interval measure. K-means clustering was conducted to refine the solution. A discriminant analysis verified the goodness of separation of the K-means algorithm. An analysis of variance (ANOVA) was used to describe the clusters. Post-hoc tests were used to determine significant differences between the means of the ANOVA. Finally, cross tables identified the socio-demographical differences between the resulting clusters.

\section{Results}

Due to the quotas set, the survey approximately represents the German population. Average age is 41 years, $49.4 \%$ are male and $50.6 \%$ are female. Regional distribution and education levels correspond with the German population. $29.9 \%$ of the respondents have a net household income of less than $€ 1,500$ per month, $28.4 \%$ have between $€ 1,500$ and $€ 2,500$ per month and $24 \%$ have $€ 2,500$ or more per month. $17.9 \%$ did not specify. Table 1 shows the percentage share in relation to the sample and its given distribution in the German population. 
Table 1. Sample characterization

\begin{tabular}{llcc}
\hline Variable & Description & $\begin{array}{c}\text { Frequency (\%) } \\
\text { Sample }\end{array}$ & $\begin{array}{c}\text { Frequency (\%) } \\
\text { Germany }\end{array}$ \\
\hline Age & 16 to 30 & 26.1 & 24.8 \\
& 31 to 50 & 42.8 & 41.2 \\
Gender & Older than 50 & 31.4 & 34 \\
& Male & 49.4 & 49.6 \\
Region & Female & 50.6 & 50.4 \\
& North & 15.8 & 15.9 \\
& South & 27.5 & 28 \\
Education level & East & 20.7 & 20.5 \\
& West & 36.1 & 35.6 \\
& No qualification & 5.2 & 7.8 \\
& Primary school & 40.2 & 36.6 \\
\multirow{2}{*}{ Net household income } & 28.2 & 28.8 \\
& Secondary school & 13.0 & 13.6 \\
& A-level & 13.4 & 13.2 \\
& University or vocational qualification & 29.9 & - \\
& Less than 1,500 & 28.4 & - \\
& 1,500-2,500 & 24.0 & - \\
\hline Source. Author & 15.7 & 0.4 \\
\hline
\end{tabular}

Source. Authors' calculation; Federal Statistical Office (2012)

First of all, the respondents were asked to provide their semantic association to images of cows by means of a semantic differential (Figure 1 and Figure 2). The pictures were taken from typical farms and discussed with experts from the industry. Especially the indoor system is presented with images coming from newly built and modern farms using cubicle housing systems.
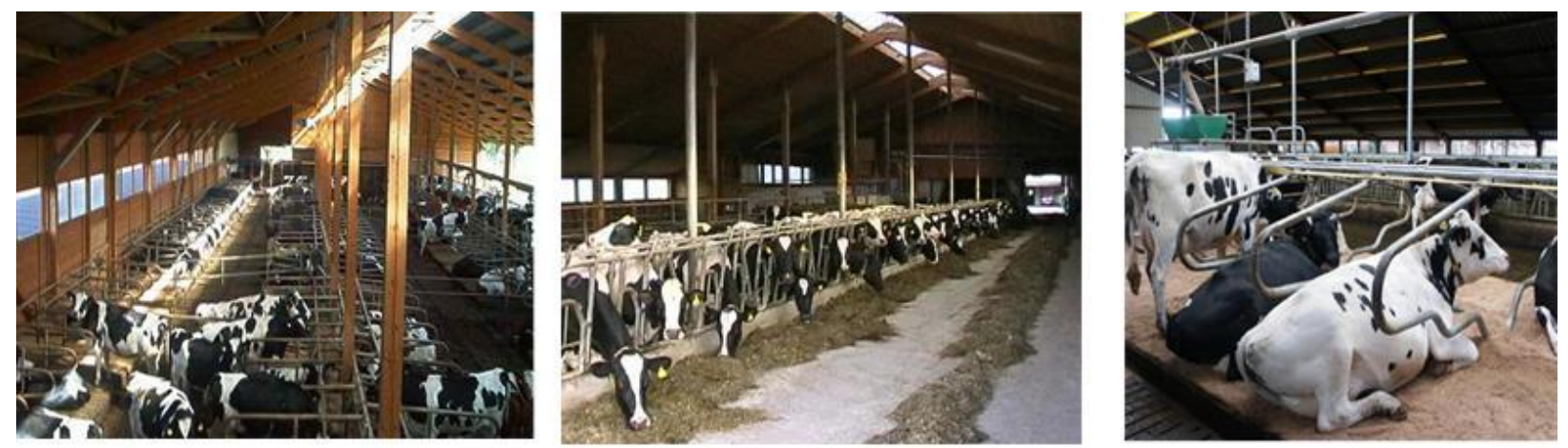

Figure 1. Presented pictures of housed systems

\footnotetext{
${ }^{14}$ Quota of the German population solely for the purposes of showing the quota (age, gender and region) used to build the sample.
} 

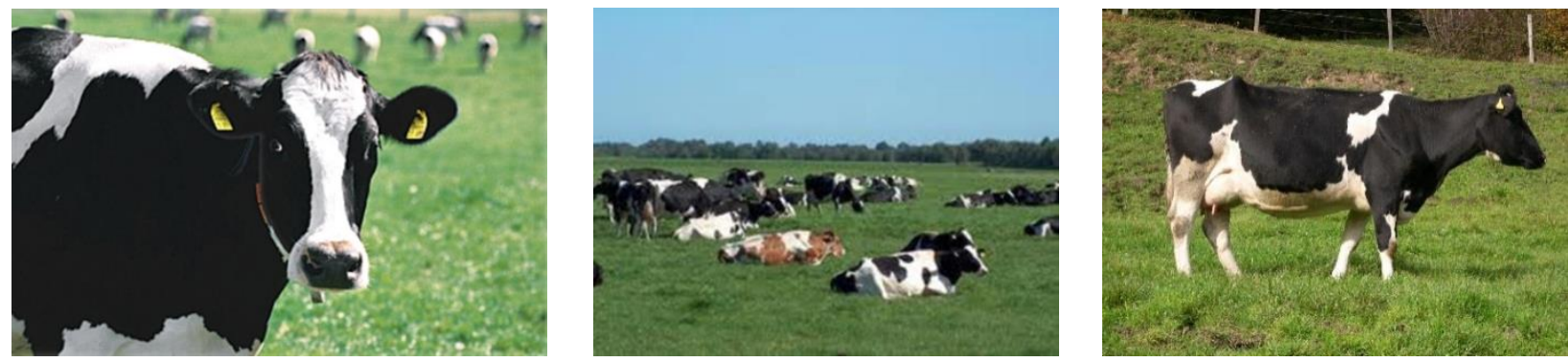

Figure 2. Images presented of outdoor systems

Tables 2 and 3 show higher mean values for positively connoted words connected with images of outdoor systems. As tables 2 and 3 show, the housed system evoked more negative connotations than did the images of the dairy cows outdoors.

Table 2. Semantic differential for fully housed systems (answers in \%)

\begin{tabular}{|c|c|c|c|c|c|c|c|}
\hline & $\begin{array}{l}\text { Very } \\
(2)\end{array}$ & $\begin{array}{l}\text { Slightly } \\
\text { (1) }\end{array}$ & $\begin{array}{c}\text { Partly / } \\
\text { partly (0) }\end{array}$ & $\begin{array}{c}\text { Slightly } \\
(-1)\end{array}$ & $\begin{array}{l}\text { Very } \\
(-2)\end{array}$ & & $\begin{array}{l}\text { Mean } \\
\text { value }\end{array}$ \\
\hline Animal friendly & 9.2 & 10.9 & 28.2 & 25.9 & 25.9 & $\begin{array}{r}\text { Cruel towards } \\
\text {-animals }\end{array}$ & -0.48 \\
\hline Healthy & 10.4 & 15.9 & 35.6 & 20.1 & 18.1 & Unhealthy & -0.20 \\
\hline Traditional & 11.0 & 14.4 & 22.8 & 20.1 & 31.8 & Industrial & -0.47 \\
\hline Modern & 26.2 & 30.4 & 28.0 & 7.1 & 8.3 & Old-fashioned & 0.59 \\
\hline $\begin{array}{r}\text { Environmentally } \\
\text {-friendly }\end{array}$ & 9.1 & 14.3 & 41.2 & 20.6 & 14.8 & $\begin{array}{r}\text { Environmentally } \\
\text {-harmful }\end{array}$ & -0.18 \\
\hline Caring & 7.4 & 10.3 & 25.0 & 27.1 & 30.1 & Loveless & -0.62 \\
\hline Close to nature & 6.9 & 7.6 & 20.6 & 24.9 & 40.0 & Unnatural & -0.84 \\
\hline
\end{tabular}

Table 3. Semantic differential for outdoor systems (answers in \%)

$\begin{array}{cccccc}\text { Very } & \text { Slightly } & \text { Partly / } & \text { Slightly } & \text { Very } & \text { Mean } \\ (2) & (1) & \text { partly (0) } & (-1) & (-2) & \text { value }\end{array}$

\begin{tabular}{|c|c|c|c|c|c|c|c|}
\hline Animal friendly & 70.4 & 17.2 & 10.0 & 1.8 & 0.6 & $\begin{array}{r}\text { Cruel towards } \\
\text { animals }\end{array}$ & 1.55 \\
\hline Healthy & 65.6 & 21.6 & 11.3 & 1.0 & 0.6 & Unhealthy & 1.5 \\
\hline Traditional & 65.2 & 21.6 & 10.3 & 2.1 & 0.9 & Industrial & 1.48 \\
\hline Modern & 27.5 & 21.4 & 34.6 & 12.5 & 4.0 & Old-fashioned & 0.56 \\
\hline $\begin{array}{r}\text { Environmentally } \\
\text {-friendly }\end{array}$ & 56.0 & 25.7 & 14.9 & 2.7 & 0.7 & $\begin{array}{r}\text { Environmentally } \\
\text {-harmful }\end{array}$ & 1.34 \\
\hline Caring & 54.7 & 26.9 & 15.4 & 2.2 & 0.8 & Loveless & 1.32 \\
\hline Close to nature & 74.2 & 15.2 & 8.9 & 0.9 & 0.8 & Unnatural & 1.61 \\
\hline
\end{tabular}

In a second step, attitudes towards the different systems were evaluated using likert scale questions, resulting in a factor analysis. According to the Kaiser-Meyer-Olkin criterion, the 
result of the factor analysis is excellent $(\mathrm{KMO}=0.929$; Kaiser 1974). Bartlett's test of sphericity is highly significant, which demonstrates that the variables are highly correlated (Backhaus et al. 2006). The survey had several goals. As the aim was to identify consumers' attitudes concerning fully housed systems and outdoor systems, the first two factors from Table 4 were chosen. As the third factor pertains to the consumer-citizen-gap, it was chosen as well. Thus, only the first three out of the resulting six factors in Table 4 entered the cluster analysis as the focal point. Adding the other factors to the cluster analysis might also have resulted in clusters being too complex. It is these three factors that are presented hereafter. The first factor includes eight items regarding pasture-raised milk. It is thus named pro pasturing. The second factor combines seven items that support fully housed systems and is thus named pro fully-housed systems. The third factor includes seven items regarding attitudes towards quality. Items loading on this factor refer to regional milk purchase, WTP for known brands, purchase of organic milk as well as environmentally-friendly and animal-friendly production standards, in addition to the items "Healthy nutrition is important to me" and "I like to try new things". All items and factor loadings are outlined in detail in Table 4. 
Table 4. Results of the factor analysis

\begin{tabular}{|c|c|}
\hline \multirow{2}{*}{\multicolumn{2}{|c|}{$\begin{array}{l}\text { Factors and Items } \\
\text { Pro pasturing }(\text { Cronbach's Alpha }=0.918 ; \% \text { of variance }=17.950)\end{array}$}} \\
\hline & \\
\hline Pasture grass is important for the good nutrition of animals. & 0.847 \\
\hline Outdoor exercise in the fresh air is important to make the animals feel comfortable. & 0.826 \\
\hline Pasture is important for our natural environment. & 0.802 \\
\hline Dairy cows at pasture are important in our agricultural landscape. & 0.799 \\
\hline Dairy cows need outdoor exercise in the fresh air. & 0.745 \\
\hline Fresh grass as feed makes animals healthier. & 0.744 \\
\hline For me, pasturing is the most natural form of dairy farming. & 0.681 \\
\hline I cannot imagine an agricultural landscape without grazing cows. & 0.666 \\
\hline \multicolumn{2}{|l|}{ Pro fully housed systems (Cronbach's Alpha $=0.833 ; \%$ of variance $=11.994)$} \\
\hline Dairy cows in indoor systems are better looked after. & 0.799 \\
\hline Animal illness will be noticed faster in indoor systems. & 0.746 \\
\hline Dairy cows in indoor systems can be fed according to requirements. & 0.741 \\
\hline Dairy cows in indoor systems are better protected against heat and cold. & 0.735 \\
\hline Dairy cows in indoor systems produce more milk and are therefore more climate-friendly. & 0.655 \\
\hline Milk can be produced more cost-effectively in indoor systems. & 0.631 \\
\hline I can understand that farmers these days do not want to push dairy cows onto pasturage every day. & 0.445 \\
\hline \multicolumn{2}{|l|}{ Quality orientation (Cronbach's Alpha $=0.809 ; \%$ of variance $=9.959)$} \\
\hline $\begin{array}{l}\text { While shopping I try to look out for products that were produced in an environmentally-friendly } \\
\text { way. }\end{array}$ & 0.703 \\
\hline While shopping I try to look out for products that were produced in an animal-friendly way. & 0.689 \\
\hline I mostly buy organic milk. & 0.680 \\
\hline For known brands, I would definitely pay a surcharge. & 0.609 \\
\hline I prefer buying milk from my region. & 0.565 \\
\hline Healthy nutrition is important to me. & 0.556 \\
\hline I like to try new things. & 0.519 \\
\hline \multicolumn{2}{|l|}{ Dairy company policy $($ Cronbach's Alpha $=0.898 ; \%$ of explained variance $=9.288$ ) } \\
\hline The dairy farm behaves in an environmentally-conscious way. & 0.814 \\
\hline The milk is from species-appropriate livestock farming. & 0.809 \\
\hline Fair milk prices for farmers in Germany. & 0.796 \\
\hline Milk in its natural state. & 0.710 \\
\hline \multicolumn{2}{|l|}{ Pragmatism $($ Cronbach's Alpha $=0.746 ; \%$ of explained variance $=6.971)$} \\
\hline If indoor housing means cheaper milk, it suits me. & 0.670 \\
\hline I have to do my shopping fast; I don't look out for differences in milk. & 0.655 \\
\hline I especially look out for low-priced milk prices. & 0.645 \\
\hline If the cows are well, indoor housing is fine. & 0.538 \\
\hline If modern cowsheds provide animals with plenty of exercise and fresh air, that is completely fine. & 0.508 \\
\hline \multicolumn{2}{|l|}{ Animal Welfare (Cronbach's Alpha $=\mathbf{0 . 6 4 4} \%$ of explained variance $=4.624)$} \\
\hline I cannot imagine that cows that are living only in a barn can feel well. & 0.669 \\
\hline For me, keeping cows indoors year-round is cruelty to animals. & 0.653 \\
\hline $\begin{array}{l}\text { KMO }(\text { Kaiser-Meyer-Olkin })=0.929 ; \text { explained total variance }=60.79 \% \\
\text { Bartlett-Test for sphericity }=16,946.484 ; \text { significance }=0.000 \\
n=1,009\end{array}$ & \\
\hline
\end{tabular}

Based on Ward's method, scree tests, a dendrogram and other practical considerations, a fourcluster solution was decided upon. K-means gave $\mathrm{F}$ values for all the cluster-forming variables that were significant at the $1 \%$ level, suggesting that the clusters were homogeneous. The average value for Eta is 0.726 , showing that there are significant differences between the cluster-forming factors and that the variance within the clusters, is negligible. Eta-squared is 0.529 ; therefore, $52.9 \%$ of the variance within the cluster-forming factors can be attributed to 
differences between the clusters. $96.8 \%$ of the cases were attributed to the same clusters by both K-means and discriminant analysis. Table 5 (see Appendix) contains the detailed results of the cluster analysis.

The four clusters can be characterized as follows: The first cluster is the second largest, with a total of 281 consumers. It has high mean values for the pro pasturing and quality orientation factors, whereas the pro fully housed systems factor has a negative mean value. This is therefore the "quality-conscious" cluster. The second cluster is the smallest one. It has no high values for any factor mean value. Therefore, it could be characterized as the "undecided" cluster. The third cluster has a size of 257 respondents. These consumers are less quality-orientated, but show positive values for the pro pasturing and pro fully housed systems factors. This cluster is named the "generalists". The fourth cluster is the largest one. It has a high value for the pro pasturing factor, but lower values for the pro fully housed systems and quality orientation factors. This is therefore the "pasture-supporters" cluster.

The results illustrate that clusters 1, 3 and 4 have the highest mean values for the pro pasturing factor. Since the third cluster also has a high score for the pro fully housed systems factor, this cluster is a less optimal target group for pasture-raised milk. The quality orientation factor is an important aspect for consumers in the first cluster, whereas consumers in the fourth cluster have less interest in the quality characteristics of the products. This difference could be explained by socio-demographic relationships. Whereas the first cluster contains significantly more women and more consumers with a higher education level, cluster number four is overrepresented by consumers from the lower income classes. This group also has significantly more consumers with only a secondary school education and significantly less with a university degree.

To discover whether the theory of the consumer-citizen-gap can be verified, the WTP for pasture-raised milk was requested in the survey. The respondents were asked to imagine that they were in front of a supermarket shelf. They saw four realistic offers of milk with the corresponding realistic prices, as follows: private label ( $€ 0.65)$, milk brand one ( $€ 0.95)$, organic milk ( $€ 1.05)$, milk brand two $(€ 1.25)$. The respondents were also shown a product dummy of pasture-raised milk. Then, they were requested to state their WTP for 1 litre pasture-raised milk. Outliers stating a WTP more than $30 \%$ of the average WTP or less than $30 \%$ of the average WTP were removed from the WTP calculation.

On average a WTP of $€ 1.04$ was stated. The WTP for 1 litre of pasture-raised milk was also calculated for each different cluster and compared to reference prices. The result for the first 
cluster is a WTP of $€ 1.13$, for the second $€ 0.98$ and respondents of the third and fourth cluster each stated a WTP of $€ 1.01$ for 1 litre of pasture-raised milk. The WTP of the first cluster is significantly higher than the WTP of the other clusters. Table 6 below gives an overview of the WTP for the four clusters.

Table 6. Results of the calculated WTP

\section{\begin{tabular}{llll}
\hline WTP for Cluster 1 & WTP for Cluster 2 & WTP for Cluster 3 & WTP for Cluster 4
\end{tabular}}
$€ 1.13$
$€ 0.98$
$€ 1.01$
$€ 1.01$

\section{Discussion}

The literature shows that there is a gap between the rising number of large farms that prefer housed systems for dairy cattle (Schleyer et al. 2013) and customers who demand milk from cows with access to pasture (Ellis et al. 2009, WSPA 2010). The survey presented reveals that consumers differ in their attitudes towards the different housing systems and in their quality orientation. As this was an approximately representative sample, the results can be transferred to the German population.

The semantic differential clearly gives an initial indication that free range systems have positive connotations whereas images of indoor systems evoke more negative emotions. A reason for that might be that grazing cows are firmly anchored images. As known in the literature, pictures can be recollected better than words (e.g. Paivio and Csapo 1973, Graber 1996). The factor analysis confirms a separated perception of housed and outdoor systems, by items loading on two different factors (pro fully housed system / pro pasturing). The positive associations of pasturing are therefore separate from the negative associations of a fully housed system. The items loading on the factor pro fully housed system are perceived primarily as technical advantages by farmers, as opposed to the emotional items loading on the factor pro pasturing.

A particularly suitable target group for pasture-raised milk is the first cluster of qualityconscious participants with a high education level, but the value attached to pasturing by the fourth cluster also makes it a suitable candidate. Both clusters demonstrate high levels of agreement with statements concerning cows having access to pasture and fresh air. They also both agree that they wish to retain dairy cattle in the landscape. 
There are, however, clear differences between the clusters regarding quality aspects. For the first cluster respondents it is important to know where the milk they purchase comes from. They have the highest agreement levels to the statements "While shopping I try to look out for products that were produced in an environmentally-friendly way." $(\mu=1.34)$ and "While shopping I try to look out for products that were produced in an animal-friendly way." $(\mu=1.38)$. These two statements are less distinctive for the fourth cluster $(\mu=0.06$ and $\mu=0.21$ ). Quality orientation is generally not an important aspect for pasture-supporters $(\mu=-0.15)$. This might be due to the fact that the fourth cluster has significantly more respondents with a lower education level and also a significantly higher proportion of respondents distributed across the two lowest income classes. Pasturing might be a very important aspect to these consumers, but when out shopping they pay less attention to food quality aspects. Price might be more important for this group as they show a lower WTP than the quality-conscious cluster. The first cluster of quality-conscious, which makes up $28.1 \%$ of the participants, can therefore be seen as the core target group. The results are congruent with the present market share of $20 \%$ of pasture-raised Danish milk (Heerwagen et al. 2013) and the calculated WTP: While the first and fourth clusters have similar attitudes towards the housing systems, the WTP of the quality-conscious cluster at $€ 1.13$ is 12 cents higher than the WTP of the pasture-supporters. The fourth cluster of pasture-supporters $(28.3 \%)$ can be seen as an extended target group given that they also prefer pasturing, but are not willing to pay such a high price for pasture-raised milk as the quality-conscious cluster. The differences between cluster 1 and cluster 4 are a strong indication of a possible consumer-citizen-gap. Both clusters show similar attitudes concerning the factor pro pasturing and pro fully housed system. But when comparing the WTP for pasture-raised milk between the clusters, it can be seen that the first cluster has a significantly higher WTP $(€ 1.13)$ than the fourth cluster $(€ 1.01)$. Thus, it can be assumed that for the fourth cluster pasturing is not an important buying motive and so does not influence the purchase decision what is also supported by their lower agreement to quality attributes.

The third cluster, the generalists $(25.7 \%)$, may also be an extended target group for pastureraised products. Statements about pasture-access are important for them, but they do not disapprove of indoor-housing as much as the other groups. In this respect they agree particularly with the statements in favour of the indoor-housing system that refer to advantages for animals (e.g., "In indoor systems, animal illness will be noticed faster."). In addition, they tend to look for animal-friendly produced products $(\mu=0.47)$. If they are informed about the gains of outdoor systems it might influence their purchasing decision. The positive attitude towards 
both housing systems $(\mu=1.18 ; \mu=1.04)$ confirms that indoor and outdoor systems are separately perceived. Consumers in the third cluster see positive aspects for indoor and outdoor systems. They seem to be open to arguments for both housing systems.

A clear market potential for pasture-raised milk is thus shown. Moreover, all groups would pay a surcharge for pasture-raised milk, which is consistent with results from previous surveys (Pirog 2004, Ellis et al. 2009, Hellberg-Bahr et al. 2012). The results mean that a financial incentive for producing pasture-raised milk and dairy products would be reasonable for producers, processors and marketers, as long as this aspect is highlighted and promoted on the product. Offering an incentive is important in developing a solid market. However, the calculated WTP has to be carefully considered due to the theory of the consumer-citizen-gap. A known gap exists between the attitudes of citizens and their actual behaviour as customers during their purchasing situations (especially with respect to animal welfare aspects in food) (Coff et al. 2008, Harvey and Hubbard 2013). Animal welfare aspects as well as environmental and quality aspects can be overlooked during the decision-making process in the supermarket as while completing the survey customers are answering as citizens who are presenting their general opinion. The two most promising target groups differ in their attitudes towards quality orientation ( $\mu=0.97$ for the first cluster and $\mu=-0.15$ for the fourth cluster) and their WTP ( $€ 1.13$ and $€ 1.01$ ), which might confirm that for some customers there is a gap in their behaviour as citizen and consumer. While the quality-conscious will also pay a premium, pasturing-supporters decide according to the price they see on the product shelf in the supermarket. This is confirmed by the significantly higher WTP of the first cluster.

\section{Conclusions}

As the results show, housed systems evoke negative connotations. In the semantic differential and the cluster analysis more than $50 \%$ of respondents consider fully housed systems problematic. Obviously, many consumers have clear preferences for pasturing. This attitude has already created a severe image problem for those keeping laying hens in cages. In Germany, today, legal guidelines forbid this type of livestock farming. In order to prevent a similar crisis, the dairy sector has to face up to this consumer perception.

At first thought it could be seen as a paradox to compensate by a higher price and used as a marketing tool the fact that dairy cattle have access to pasture, given that this was a norm only a few decades ago. The changes of structure in dairy farming (less use of grassland in dairy farming; see above) have caused the dairy sector to face up to these new issues. The conduct- 
ed consumer survey shows that pasturing is an important issue for customers and can be used as a sales argument for a relatively large group of customers.

Today, only a few countries, such as the Netherlands, have consistent standards for dairy products labelled as pasture-raised. But it is only fixed standards for the term pasture-raised milk that can guarantee consumers will not feel deluded. Honest and transparent standards and an appropriate labelling system for pasture-raised milk must therefore be built up in the near future to target cluster one: a consumer group of $28.1 \%$ of the German population which is characterized by a significantly higher WTP for pasture-raised milk. Otherwise, farmers may tend to give up on pasture-grazing for their dairy cattle due to higher economies of scale of indoor housing systems. Additionally, a study by Kehlbacher et al. (2012) examined the fact that information about certification has a positive influence on WTP. It is therefore important to live up to the demands of consumers and also to the practicalities of farmers. Taking all market participants into account, a solid system can be generated that retains and builds upon the grazing system. Given these conditions, the market potential demonstrated encourages farmers and the dairy-products sector to highlight and promote the positive aspects of pasture-raised milk.

\section{Limitations}

Due to the discrepancy between consumer and citizen, future WTP research has to be verified by demonstrated preferences, e.g., in a supermarket test. Furthermore, the results are only valid for the German population and no comparable research yet exists for housing systems. Further research in Germany and in additional countries therefore needs to be carried out to discover consistent or contrary results. The survey also provides hints on the importance of food source for dairy cows in terms of grass and the fatty acid composition of milk in terms of omega-3 fatty acids. More detailed research is necessary to evaluate their importance on a purchasing decision. For example, in Austria instead of pasture-raised milk, a prominent marketing trend in the milk sector is hay milk ("Heumilch"), a label that guarantees the abandonment of silage fodder.

\section{References}

Akerlof, G. A. 1970. The Market for Lemons, Quality Uncertainty and the Market Mechanism. Quarterly Journal of Economics 84 (3): 488-500.

Aertsens, J., W. Verbeke, K. Mondelaers and G. V. Huylenbroeck. 2009. Personal determinants of organic food consumption: a review. British Food Journal 111 (10): 1140-1167. 

implications for the marketing of pasture-raised milk

Backhaus, K., B. Erichson, W. Plinke and R. Weiber. 2006. Multivariate Analysemethoden: Eine anwendungsorientierte Einführung. 11th Edition. Springer, Berlin.

Baur, I., M. Dobricki and M. Lips. 2010. Einstellung zur Hochleistungs- und Vollweidestrategie. Agrarforschung Schweiz 1 (9): 326-333.

Bickel, M., Mühlraht, D. and K. Zander. 2009. Kaufmotive und Zahlungsbereitschaften für Erzeuger-Fair-Milch-Produkte der Upländer Baurnmolkerei. In: Mayer, J., T. Alföldi, F. Leiber, D. Dubois, P. Fried, F. Heckendorn, ... and H. Willer (Eds.). 10. Wissenschaftstagung Ökologischer Landbau: 10-14. Berlin.

Brade, W. 2012. Vor- und Nachteile der Weidehaltung von hochleistenden Milchkühen. Berichte über Landwirtschaft 90 (3): 447-466.

Caswell, J. A. and S. Joseph. 2008. Consumer Demand for Quality: Major Determinant for Agricultural and Food Trade in the Future? Journal of International Agricultural Trade and Development 4 (1): 99-116.

Coff, C., M. Korthals and D. Barling. 2008. Ethical Traceability and Informed Food Choice. Ethical Traceability and Communicating Food, edited by Coff, C., D. Barling, M. Korthals and N. Thorkild, 1-14. Springer, Dordrecht.

Deimel, I., S. Rumm and B. Schulze. 2012. Öffentlichkeitsarbeit der Verdelungsbranche: Eine empirische Analyse der Wahrnehmung von Landwirten und Verbrauchern im Licht der Konflikt- und Glaubwürdigkeitsforschung. Schriftenreihe der Rentenbank Band 28 - Veredelungsstandort Deutschland: 51-73.

Ellis, K. A., K. Billington, B. McNeil and D. E. F. McKeegan. 2009. Public opinion on UK milk marketing and dairy cow welfare. Animal Welfare 18 (3): 267-282.

FAO (Food and Agriculture Organization of the United States). No date. Chapter 2: Global Dairy Sector: Status and Trends. http://www.fao.org/docrep/012/i1522e/i1522e02.pdf [accessed November 16, 2013].

Fearne, A. and D. Lavelle. 1996. Segmenting the UK egg market: results of a survey of consumer attitudes and perceptions. British Food Journal 98 (1): 7-12.

Federal Statistical Office. 2011. Land- und Forstwirtschaft, Fischerei, Wirtschaftsdünger, Stallhaltung, Weidehaltung Landwirtschaftszählung/Agrarstrukturerhebung 2010. Statistisches Bundesamt Wiesbaden Fachserie 3, Book 6.

Federal Statistical Office. 2012. "Statistisches Jahrbuch. Deutschland und Internationales", Wiesbaden, available at: https://www.destatis.de/DE/Publikationen/StatistischesJahr buch/StatistischesJahrbuch2012.pdf?_blob=publicationFile. [accessed 29 July 2013].

Graber, D. 1996. Say it with pictures. American Academy of Political and Social Science 546 (1): 85-96.

Harvey, D. and C. Hubbard. 2013. Reconsidering the political economy of farm animal welfare: An anatomy of market failure. Food Policy 38 (2013): 105-114.

Heerwagen, L. R., T. Christensen, and P. Sandøe. 2013. The Prospect of Market-Driven Improvements in Animal Welfare: Lessons from the Case of Grass Milk in Denmark. Animals 3 (2): 499-512.

Hellberg-Bahr, A., N. Steffen and A. Spiller. 2012. Marketingpotentiale für Weidemilch. Jahrbuch der Österreichischen Gesellschaft für Agrarökonomie 21 (1): 3-12. 
Hörning, B. 2009. Beurteilung der Tiergerechtheit der ,Kleingruppenhaltung' von Legehennen. http://mulewf.rlp.de/fileadmin/mufv/img/inhalte/tiere/Gutachten_LH_ Hoering_2009.pdf [accessed July 22, 2014].

Kaiser, H. F. 1974. An index of simplicity. Psychometrika 39 (1): 31-36.

Kehlbacher, A., R. Bennett and K. Balcombe. 2012. Measuring the consumer benefits of improving farm animal welfare to inform welfare labelling. Food Policy 37 (6): 627-633.

Laepple, D., T. Hennessy and M. O’Donovan. 2012. Extended grazing: A detailed analysis of Irish dairy farms. Journal of Dairy Science 95 (1): 188-195.

McEachern, M.G. and M. J. A. Schröder. 2002. The Role of Livestock Production Ethics in Consumer Values towards Meat. Journal of Agricultural and Environmental Ethics 15 (2): 221-237.

McGarry Wolf, M., L. J. Butler, A. J. Martin and J. D. Foltz. 2009. Factors influencing the purchase decision for milk labelled rBST-free and organic. Journal of Food Distribution Research 40 (1): 187-191.

Ostermann-Palz, B. and C. Stöcker. 2013. Zurück ins Grüne? Elite 4: 12-15.

Padel, S. and C. Foster. 2005. Exploring the Gap between Attitudes and Behaviour: Understanding why consumers buy or do not buy organic food. British Food Journal 107 (8): 606-625.

Paivio, A. and K. Csapo. 1973. Picture superiority in free recall: Imagery or dual coding? Cognitive Psychology 5 (3): 176-206.

Pearson, D., Henryks, J. and H. Jones. 2011. Organic Food: What we know (and do not know) about consumers. Renwable Agriculture and Food Systems 26 (2): 171-177.

Pirog, R. 2004. Consumer Perceptions of Pasture-raised Beef and Dairy Products: An Internet Consumer Study. Leopold Center, Iowa State University.

Plaßmann, S. and U. Hamm. 2009. Kaufbarriere Preis? - Analyse von Zahlungsbereitschaft und Kaufverhalten bei Öko-Lebensmitteln. Final report 06OE119. Universität Kassel, Germany. http://orgprints.org/15745/1/15745-06OE119-uni_kassel-hamm-2009kaufbarriere_preis.pdf [accessed November 16, 2013].

Reijs, J. W., C. H. G. Daatselaar, J. F. M. Helming, J. Jager and A. C. G. Beldman. 2013. Grazing dairy cows in North-West Europe. LEI Report 2013-001. Wageningen.

Schleyer, A., W. Lorleberg and M. Mergenthalter. 2013. Steigerung der landwirtschaftlichen Wertschöpfung durch Produkte aus Weidehaltungssystemen. Final report 30. Fachhochschule Südwestfalen, Soest, Germany. http://www4.fhswf.de/media/downloads/fbaw_1/download_1/lorleberg/Weidemilch_Abschlussbericht_13 1008_final.pdf [accessed November 17, 2013].

Spörndly, E. 2012. Grazing - A challenge in intensive milk production. http://www.slu.se/ Documents/externwebben/vhfak/LEARN/e_sporndly_cdm_nov_2012.pdf [accessed November 20, 2013].

Steinwidder, A., W. Starz, L. Podstatzky, L. Kirner, E. M. Pötsch, R. Pfister and M. Gallnböck. 2009. Ergebnisse zur saisonalen Low-Input Vollweidehaltung von Milchkühen im österreichischen Berggebiet. Conference Proceeding of 10th Wissenschaftstagung Ökologischer Landbau 2: 62 - 65. 
Tsakiridou, E., C. Boutsouki, Y. Zotos and K. Mattas. 2007. Attitudes and behavior towards organic products: an exploratory study. International Journal of Retail \& Distribution Management 36 (2): 158 - 175.

WSPA (World Society for the Protection of Animals). 2010. Three in five milk buyers say they would never buy milk produced in large indoor dairies. http://www.ipsosmori.com/researchpublications/researcharchive/2653/

Three-in-five-milk-buyers-say-they-would-never-buy-milk-produced-in-large-indoordairies.aspx [accessed November 16, 2013].

Zander, K. and U. Hamm. 2010. Welche zusätzlichen ethischen Eigenschaften ökologischer Lebensmittel interessieren Verbraucher? German Journal of Agricultural Economics 59 (4): $246-257$. 


\section{Appendix}

Table 5. Results of the cluster analysis

\begin{tabular}{|c|c|c|c|c|c|}
\hline & $\begin{array}{c}\text { Cluster } 1 \\
\text { Quality-Conscious }\end{array}$ & $\begin{array}{c}\text { Cluster } 2 \\
\text { Undecided }\end{array}$ & $\begin{array}{c}\text { Cluster } 3 \\
\text { Generalists }\end{array}$ & $\begin{array}{c}\text { Cluster } 4 \\
\text { Pasture-Supporters }\end{array}$ & Sample \\
\hline Cluster size absolute and in \% & $\begin{array}{c}281(28.1 \%) \\
\text { Mean value } \\
(\mathrm{SD}) \\
\text { [factor value] }\end{array}$ & $\begin{array}{l}179(17.9 \%) \\
\text { Mean value } \\
\quad(\text { SD }) \\
\text { [factor value] }\end{array}$ & $\begin{array}{l}257(25.7 \%) \\
\text { Mean value } \\
\quad(\text { SD }) \\
\text { [factor value] }\end{array}$ & $\begin{array}{c}283(28.3 \%) \\
\text { Mean value } \\
(\mathrm{SD}) \\
\text { [factor value] }\end{array}$ & $\begin{array}{l}\text { Mean } \\
\text { value } \\
(\mathrm{SD})\end{array}$ \\
\hline Factor 1: Pro Pasturing ${ }^{1}$ & $\begin{array}{l}1.57 \\
(0.561) \\
{[0.36]} \\
\end{array}$ & \begin{tabular}{c|c|}
0.2 \\
$(0.855)$ \\
{$[-1.54]$} \\
\end{tabular} & $\begin{array}{l}1.18 \\
(0.711) \\
{[0.05]} \\
\end{array}$ & $\begin{array}{l}1.55 \\
(0.563) \\
{[0.57]} \\
\end{array}$ & \\
\hline $\begin{array}{l}\text { For me, pasturing is the most natural form } \\
\text { of dairy farming. } 4\end{array}$ & $\begin{array}{c}1.54^{\text {ad }} \\
(0.708)\end{array}$ & $\begin{array}{c}0.28 \\
(0.895)\end{array}$ & $\begin{array}{c}1.16 \\
(0.784)\end{array}$ & $\begin{array}{r}1.56^{\mathrm{ad}} \\
(0.633)\end{array}$ & $\begin{array}{l}1.22 \\
(0.880)\end{array}$ \\
\hline $\begin{array}{l}\text { I cannot imagine an agricultural landscape } \\
\text { without grazing cows. }\end{array}$ & $\begin{array}{l}1.4^{\mathrm{ad}} \\
(0.765)\end{array}$ & $\begin{array}{l}0.02 \\
(0.840)\end{array}$ & $\begin{array}{c}0.92 \\
(0.879)\end{array}$ & $\begin{array}{l}1.36^{\mathrm{ad}} \\
(0.766)\end{array}$ & $\begin{array}{l}1.02 \\
(0.955)\end{array}$ \\
\hline $\begin{array}{l}\text { Fresh grass as feed makes animals } \\
\text { healthier. }\end{array}$ & $\begin{array}{r}1.51^{\mathrm{ad}} \\
(0.683)\end{array}$ & $\begin{array}{l}0.15 \\
(0.771)\end{array}$ & $\begin{array}{l}1.2 \\
(0.693)\end{array}$ & $\begin{array}{r}1.42^{\mathrm{ad}} \\
(0.663)\end{array}$ & $\begin{array}{c}1.16 \\
(0.851)\end{array}$ \\
\hline $\begin{array}{l}\text { Dairy cows need outdoor exercise in the } \\
\text { fresh air. }{ }^{4}\end{array}$ & $\begin{array}{c}1.66^{\mathrm{ad}} \\
(0.632)\end{array}$ & $\begin{array}{c}0.26 \\
(0.833)\end{array}$ & $\begin{array}{l}1.18 \\
(0.690)\end{array}$ & $\begin{array}{c}1.6^{\mathrm{ad}} \\
(0.582)\end{array}$ & $\begin{array}{l}1.27 \\
(0.844)\end{array}$ \\
\hline $\begin{array}{l}\text { Outdoor exercise in the fresh air is } \\
\text { important to make the animals feel } \\
\text { comfortable. }\end{array}$ & $\begin{array}{c}1.7^{\mathrm{ad}} \\
(0.506)\end{array}$ & $\begin{array}{c}0.36 \\
(0.796)\end{array}$ & $\begin{array}{c}1.34 \\
(0.614)\end{array}$ & $\begin{array}{r}1.72^{\mathrm{ad}} \\
(0.489)\end{array}$ & $\begin{array}{l}1.37 \\
(0.771)\end{array}$ \\
\hline $\begin{array}{l}\text { Pasture grass is important for the proper } \\
\text { nutrition of animals. } 4\end{array}$ & $\begin{array}{l}1.69^{\mathrm{ad}} \\
(0.494)\end{array}$ & $\begin{array}{c}0.28 \\
(0.762)\end{array}$ & $\begin{array}{c}1.28 \\
(0.677)\end{array}$ & $\begin{array}{l}1.63^{\mathrm{ad}} \\
(0.539)\end{array}$ & $\begin{array}{l}1.32 \\
(0.794)\end{array}$ \\
\hline $\begin{array}{l}\text { Dairy cows at pasture are important in our } \\
\text { agricultural landscape. } 4\end{array}$ & $\begin{array}{c}1.5^{\mathrm{ad}} \\
(0.628)\end{array}$ & $\begin{array}{l}0.07 \\
(0.768)\end{array}$ & $\begin{array}{l}1.15 \\
(0.760)\end{array}$ & $\begin{array}{r}1.51^{\mathrm{ad}} \\
(0.662)\end{array}$ & $\begin{array}{l}1.16 \\
(0.877)\end{array}$ \\
\hline $\begin{array}{l}\text { Pasture is important for our natural } \\
\text { environment. }\end{array}$ & $\begin{array}{r}1.59^{\mathrm{ad}} \\
(0.633)\end{array}$ & $\begin{array}{l}0.2 \\
(0.794)\end{array}$ & $\begin{array}{c}1.22 \\
(0.744)\end{array}$ & $\begin{array}{r}1.59^{\mathrm{ad}} \\
(0.618)\end{array}$ & $\begin{array}{l}1.25 \\
(0.859)\end{array}$ \\
\hline Factor 2: Pro Fully Housed Systems ${ }^{2}$ & $\begin{array}{l}-0.58 \\
(0.706) \\
{[-0.62]} \\
\end{array}$ & $\begin{array}{c}-0.06 \\
(0.692) \\
{\left[-0.24^{\text {bd }}\right]}\end{array}$ & $\begin{array}{c}1.04 \\
(0.506) \\
{[1.27]} \\
\end{array}$ & $\begin{array}{c}-0.31 \\
(0.718) \\
{\left[-0.36^{\text {bd }}\right]}\end{array}$ & \\
\hline $\begin{array}{l}\text { Milk can be produced more cost-effectively } \\
\text { in indoor systems. }\end{array}$ & $\begin{array}{l}-0.36 \\
(1.247)\end{array}$ & $\begin{array}{l}0.22^{\text {bd }} \\
(1.083)\end{array}$ & $\begin{array}{c}1.25 \\
(0.979)\end{array}$ & $\begin{array}{l}0.17^{\mathrm{bd}} \\
(1.289)\end{array}$ & $\begin{array}{l}0.30 \\
(1.314)\end{array}$ \\
\hline $\begin{array}{l}\text { I can understand that farmers these days do } \\
\text { not want to push dairy cows onto pasturage } \\
\text { every day. }\end{array}$ & $\begin{array}{l}-0.27^{\mathrm{ab} ; \mathrm{ad}} \\
(1.050)\end{array}$ & $\begin{array}{l}-0.08^{\mathrm{ab} ; \mathrm{bc}} \\
(0.878)\end{array}$ & $\begin{array}{l}0.51^{\mathrm{bc}} \\
(0.977)\end{array}$ & $\begin{array}{l}-0.27^{\mathrm{ad}} \\
(1.059)\end{array}$ & $\begin{array}{l}-0.04 \\
(1.053)\end{array}$ \\
\hline $\begin{array}{l}\text { Dairy cows in indoor systems produce more } \\
\text { milk and are therefore more climate- } \\
\text { friendly. }\end{array}$ & $\begin{array}{l}-1.03^{\mathrm{ad}} \\
(0.862)\end{array}$ & $\begin{array}{l}-0.24 \\
(0.852)\end{array}$ & $\begin{array}{l}0.45 \\
(1.204)\end{array}$ & $\begin{array}{l}-0.96^{\mathrm{ad}} \\
(0.751)\end{array}$ & $\begin{array}{l}-0.50 \\
(1.115)\end{array}$ \\
\hline $\begin{array}{l}\text { Dairy cows in indoor systems are better } \\
\text { looked after. }\end{array}$ & $\begin{array}{l}-0.71 \\
(0.889)\end{array}$ & $\begin{array}{l}-0.03 \\
(0.885)\end{array}$ & $\begin{array}{c}1.33 \\
(0.966)\end{array}$ & $\begin{array}{l}-0.36 \\
(1.071)\end{array}$ & $\begin{array}{l}0.02 \\
(1.243)\end{array}$ \\
\hline $\begin{array}{l}\text { Dairy cows in indoor systems can be fed } \\
\text { according to requirements. }\end{array}$ & $\begin{array}{l}-0.86^{\mathrm{ad}} \\
(0.891)\end{array}$ & $\begin{array}{l}-0.22 \\
(0.872)\end{array}$ & $\begin{array}{l}0.91 \\
(1.128)\end{array}$ & $\begin{array}{l}-0.72^{\mathrm{ad}} \\
(0.951)\end{array}$ & $\begin{array}{l}-0.26 \\
(1.200)\end{array}$ \\
\hline $\begin{array}{l}\text { Dairy cows in indoor systems are better } \\
\text { protected against heat and cold. }{ }^{5}\end{array}$ & $\begin{array}{l}-0.51 \\
(1.014)\end{array}$ & $\begin{array}{l}-0.12^{\text {bd }} \\
(0.890)\end{array}$ & $\begin{array}{c}1.33 \\
(0.951)\end{array}$ & $\begin{array}{l}-0.02^{\text {bd }} \\
(1.159)\end{array}$ & $\begin{array}{c}0.16 \\
(1.239)\end{array}$ \\
\hline $\begin{array}{l}\text { Animal illness will be noticed faster in } \\
\text { indoor systems. } 5\end{array}$ & $\begin{array}{l}-0.35 \\
(1.118)\end{array}$ & $\begin{array}{l}0.04^{\text {bd }} \\
(0.982)\end{array}$ & $\begin{array}{l}1.46 \\
(0.877)\end{array}$ & $\begin{array}{l}-0.02^{\text {bd }} \\
(1.251)\end{array}$ & $\begin{array}{c}0.27 \\
(1.291)\end{array}$ \\
\hline
\end{tabular}


Table 5. Continued

\begin{tabular}{|c|c|c|c|c|c|}
\hline Factor 3: Quality Orientation ${ }^{3}$ & $\begin{array}{l}0.97 \\
(0.621) \\
{[0.93]}\end{array}$ & $\begin{array}{l}-0.09 \\
(0.808) \\
{\left[-0.1^{\mathrm{bc}}\right]}\end{array}$ & $\begin{array}{l}0.27 \\
(0.834) \\
{\left[0.06^{\mathrm{bc}}\right]}\end{array}$ & $\begin{array}{l}-0.15 \\
(0.675) \\
{[-0.88]}\end{array}$ & \\
\hline 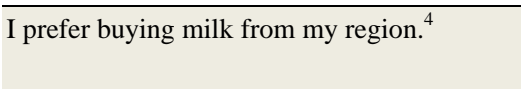 & $\begin{array}{l}0.78 \\
(1.030)\end{array}$ & $\begin{array}{l}-0.1^{\text {bd }} \\
(1.083)\end{array}$ & $\begin{array}{l}0.34 \\
(1.139)\end{array}$ & $\begin{array}{l}-0.29^{\text {bd }} \\
(1.133)\end{array}$ & $\begin{array}{l}0.20 \\
(1.178)\end{array}$ \\
\hline $\begin{array}{l}\text { For known brands, I would definitely pay a } \\
\text { surcharge. }{ }^{4}\end{array}$ & $\begin{array}{c}0.39 \\
(1.182)\end{array}$ & $\begin{array}{l}-0.52 \\
(1.088)\end{array}$ & $\begin{array}{l}-0.23 \\
(1.180)\end{array}$ & $\begin{array}{l}-0.84 \\
(1.015)\end{array}$ & $\begin{array}{l}-0.28 \\
(1.219)\end{array}$ \\
\hline I mostly buy organic milk. ${ }^{4}$ & $\begin{array}{l}-0.1 \\
(1.252)\end{array}$ & $\begin{array}{l}-0.74^{\mathrm{bc}} \\
(1.098)\end{array}$ & $\begin{array}{l}-0.94^{\mathrm{bc}} \\
(1.079)\end{array}$ & $\begin{array}{l}-1.49 \\
(0.698)\end{array}$ & $\begin{array}{l}-0.84 \\
(1.165)\end{array}$ \\
\hline Healthy nutrition is important to me. ${ }^{4}$ & $\begin{array}{l}1.6 \\
(0.533)\end{array}$ & $\begin{array}{l}0.31 \\
(0.749)\end{array}$ & $\begin{array}{l}0.96 \\
(0.706)\end{array}$ & $\begin{array}{l}0.73 \\
(0.836)\end{array}$ & $\begin{array}{l}0.95 \\
(0.844)\end{array}$ \\
\hline I like to try new things. ${ }^{4}$ & $\begin{array}{c}1.39 \\
(0.700)\end{array}$ & $\begin{array}{l}0.24 \\
(0.785)\end{array}$ & $\begin{array}{l}0.84 \\
(0.827)\end{array}$ & $\begin{array}{c}0.56 \\
(0.836)\end{array}$ & $\begin{array}{c}0.81 \\
(0.889)\end{array}$ \\
\hline $\begin{array}{l}\text { While shopping I try to look out for } \\
\text { products that were produced in an } \\
\text { environmentally-friendly way. }{ }^{4}\end{array}$ & $\begin{array}{c}1.34 \\
(0.700)\end{array}$ & $\begin{array}{l}0.12^{\mathrm{bd}} \\
(0.769)\end{array}$ & $\begin{array}{l}0.45 \\
(0.849)\end{array}$ & $\begin{array}{r}0.06^{\mathrm{bd}} \\
(0.823)\end{array}$ & $\begin{array}{c}0.53 \\
(0.947)\end{array}$ \\
\hline $\begin{array}{l}\text { While shopping I try to look out for } \\
\text { products that were produced in an animal- } \\
\text { friendly way. }{ }^{4}\end{array}$ & $\begin{array}{l}1.38 \\
(0.668)\end{array}$ & $\begin{array}{r}0.08^{\text {bd }} \\
(0.788)\end{array}$ & $\begin{array}{l}0.47 \\
(0.821)\end{array}$ & $\begin{array}{r}0.21^{\text {bd }} \\
(0.894)\end{array}$ & $\begin{array}{l}0.58 \\
(0.950)\end{array}$ \\
\hline
\end{tabular}

All results are significant at the $0.1 \%$ level; $\mathrm{n}=1,000 ; \mathrm{SD}=$ standard deviation

${ }^{1}$ Min. $=-5.32 ;$ Max. $=2.07$

${ }^{2}$ Min. $=-2.76 ;$ Max. $=2.35$

${ }^{3}$ Min. $=-3.95 ;$ Max. $=3.13$

${ }^{4}$ Scale from $+2=$ "I totally agree" to $-2=$ "I totally disagree"

${ }^{5}$ Scale from $+2=$ "I find it very convincing" to $-2=$ "I do not find it convincing at all"

${ }^{6} \mathrm{n}=1,000-1,009$

a, b, c, d: If the values in one row are marked with the same letters, the difference between the clusters is not significant (Tamhane's/Scheffe's post hoc test) 


\section{Teil III: Marketing strategies for niche products}

III.2 Consumer preferences for high welfare meat in Germany: self-service counter or service counter?

Autoren: Ramona Weinrich, Sarah Kühl, Annabell Franz, Achim Spiller

Veröffentlicht bei International Journal on Food Systems Dynamics, 2015: 6 (1), 32-49 

counter?

\begin{abstract}
Many people view animal welfare standards in the agricultural industry as critical and some consumers would prefer to buy high welfare meat. In order to successfully introduce high welfare meat products onto the market, some important marketing decisions must be made. Due to limited shelf space in retail outlets, niche products like high welfare meat cannot be placed both at the self-service counter and at the service counter. In order to analyze where to place it best an online survey of 642 German consumers was conducted. By means of factor and cluster analyses, consumers' animal welfare attitudes and their preference for a point of purchase were combined. The different target groups were joint using cross tabulation analysis. The results reveal that consumers in the target group show a more positive attitude to the service counter.
\end{abstract}

Keywords: Service counter; self-service counter; retail; animal welfare; consumer research

\title{
Introduction
}

Meat is one of the most important animal products throughout the world (Godfray et al., 2010). However, demand is not on the increase in Europe. In countries like Germany, France or Switzerland, the demand for meat has stagnated or is even decreasing (FAO 2009). Reasons may be, e.g. the perception of risk caused by several food scandals or an increasing awareness of animal welfare standards (Lippke and Sniehotta, 2003; Spiller and Schulze, 2008). Nowadays, consumers pay more attention to information such as the origin and processing details of the meat they buy (Röhr et al., 2005). Many people view animal welfare standards in the agricultural industry as critical (Verbeke and Viaene, 1999) and consumer surveys reveal that consumers would prefer to buy high welfare meat ${ }^{15}$, with some target groups willing to pay a supplement (Lagerkvist and Hess, 2011). Nevertheless, for most customers the main factor driving sales of fresh and processed meat such as ham or sausage is price and not quality (de Jonge and van Trijp, 2013). The high price of animal welfare or organic meat is often identified as being the main reason why the market share of these types of products is only marginal (McEachern and Schröder, 2002; Padel and Foster, 2005; Plaßmann and Hamm, 2009).

\footnotetext{
${ }^{15}$ By the term high welfare meat we mean meat that is produced to higher standards than legal minimum.
} 
High welfare meat is already available in the UK, the Netherlands, Switzerland, the USA and Germany and is distinguished by special labels but it is (still) a niche market in most countries. Looking more closely at Germany, the demand for such products is estimated at $20 \%$ by consumer surveys (Schulze et al., 2008a). There are already several different labels for high welfare meat, e.g. the label "Tierschutz-kontrolliert" (launched 2012) or the label "Für mehr Tierschutz" (launched 2013), but the market share is low - for both fresh meat and even lower for processed meat. This demonstrates how challenging it is to launch these kinds of products into retail.

In order to successfully introduce high welfare meat products onto the market, some important marketing decisions must be made to meet consumers' needs and thus establish an efficient range of products (Amine and Cadenat, 2003). One central question is that of where to best place high welfare meat: at the service counter or at the self-service counter? In most countries both of these distribution channels are employed in meat marketing, but due to limited shelf space in supermarkets, typically it is not possible to use both channels for the same product. Product proliferation results in a larger variety of products than is possible to accommodate on the shelves (Carlotti et al., 2006). Retailers must decide whether these products will improve profit margins; therefore, it is crucial to place products as good as possible (Hübner and Kuhn, 2012). Thus, it seems obvious that the retail sector will not be able to introduce high welfare meat products through both channels - especially because shelf space on a service counter or in refrigerated display cases is even more limited (FTC, 2003).

Consequently, retailers that offer meat and meat products via both counters must decide which channel will be more appropriate for high welfare fresh and processed meat. The two counters have different target groups and different images in the minds of consumers, with the service counter usually being associated with premium food quality (Schulze and Spiller, 2007). Furthermore, meat at the self-service counter is often less expensive than at the service counter. Another issue is that spoilage rates are higher at the self-service counter than at the service counter as sales figures are lower (personal communications with retailers).

This paper makes a particular contribution to improving the introduction of high welfare meat as a niche product in retail. An online survey of 642 German consumers was conducted to find out at which counter consumers would rather buy high welfare meat. Data on consumers' animal welfare attitudes and their preferred point of purchase were combined. This information will help retailers identify the optimal placement for high welfare meat in order to achieve the best sales. The results are not only important for German retailers and marketers 

counter?

as there are also both distribution channels in other countries as well. Furthermore, the introduction of animal welfare is a main topic in many Northwestern European countries.

After presenting the current state of research two hypotheses will be derived. In the then following sections, the methodology and the results will be described. Hereafter, a discussion section follows. The article ends with conclusions.

\section{State of research}

\section{Points of sale for meat}

Meat can be bought at different points of sale. In nearly all OECD countries, and also in more and more developing countries, the main distribution channel for fresh meat is the supermarket (Reardon, 2010): In Germany, the importance of supermarkets for meat sales is increasing steadily so that the share is now over $80 \%$. Specialized shops like butchers or open markets are decreasing (LfL 2013). A similar pattern can be seen in other countries. In Canada, supermarkets play even a more important role. $93 \%$ of Canadian meat consumers buy their meat mostly in supermarkets, while only $10 \%$ buy meat at the butcher's (ALMA, 2012). Roerink (2013) observes in her study of 1,452 respondents a similar pattern for consumers in the USA. Reasons for the trend towards buying meat from the supermarket are multidimensional. Many customers in different countries think meat from the butcher is more expensive and that a more limited range of cuts of meat is offered than at the supermarket (Ngapo et al., 2003). Additionally, it is quicker and more convenient to buy meat at the same establishment as the rest of the products (the "one stop shop", Maruyama and Wu, 2014). In many other countries, in particular developing countries, the supermarket is gaining more and more importance (cf. "supermarket revolution", Reardon et al., 2010), as seen e.g. in Argentina (Rodriguez et al., 2002), this supports the increasing importance of this distribution channel. Figure 1 shows the three most important points of sale for meat in most industrial countries the supermarket being the most important one, especially in Germany.

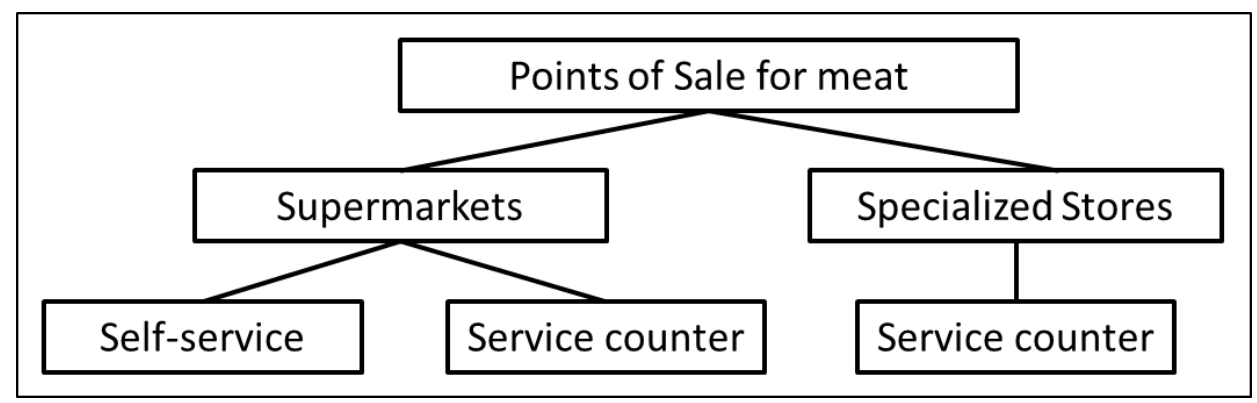

Figure 1. Points of sale for meat

Source: own presentation 
The differentiation of points of sale is driven by different types of consumers. Grunert (2006) analyzes different trends in the consumption of meat and buying behavior for meat and defines one trend as "fast and efficient shopping in supermarkets". Another mentioned trend is "the buying of information-intensive specialized products in specific retail outlets" (ibid). For consumers who do not attach high importance to the meat they purchase, who are primarily focused on price (de Jonge and van Trijp, 2013) and are guided by their own experience (Verbeke and Vackier, 2004), pre-packaged meat at the self-service counter is a good concept: Here, they can choose their meat on their own and do not have to wait for service staff. This kind of purchase is quicker, thus the most mentioned argument for the self-service counter is the economy of time (ALMA, 2012). Besides the time pressure factor, the lower price anticipated for meat, both fresh and processed meat, at the self-service counter is another argument for many consumers (Schulze and Spiller, 2007; Weyer, 2005).

In addition to a self-service counter, many supermarkets also offer meat at the service counter. In Germany, both channels are used and valued by customers but the proportion of service counter sales is declining. Nowadays, fresh meat from the service counter has a decreasing market share of $45 \%$. It is even less for processed meat (25\%) (LfL, 2013; Figure 2 and 3). A survey by Smith and Burns (1997) in the USA concluded that only $4 \%$ of respondents buy meat exclusively at the service counter. They therefore reason that self-service counters and service counters cannot be substituted for one another but that consumers use them in a supplementary manner. Schulze and Spiller (2007) characterized German consumers of meat into the typical self-service counter buyer and the typical service counter buyer. While the time factor is an argument for the self-service counter as already stated by ALMA (2012), the better quality of meat anticipated at the service counter (Weindlmaier, 1980) as well as advice from the counter staff (Balling, 1990), e.g. on how to prepare the meat, are motives for consumers to buy their meat at the service counter. Furthermore, Schulze and Spiller (2007) found that consumers in the service counter target group receive a higher income and are not as price-sensitive as respondents who prefer to shop at the self-service counter.

\section{Distribution of fresh and processed meat at retail counters}

Literature shows that a target group for animal welfare products exists and that consumers are willing to pay a supplement (Lagerkvist and Hess, 2011). Of course, not all consumers are willing and able to pay a higher price for meat. Therefore, supermarkets also need to offer conventional meat at lower prices and will not be able to concentrate on high welfare meat. If they did, they would run the risk of losing price-oriented consumers, as they would have to 

counter?

shift shelf space away from conventional products to accommodate high welfare meat products. Due to space constraints, in most cases supermarkets will also not offer high welfare meat products at two counters, the self-service counter and the service counter (Carlotti et al., 2006). Currently, neither fresh nor processed high welfare meat is sold at the service counter on a large scale in Germany.

So far, little research has been done to answer the question of where high welfare meat should be best placed. Only one survey from Germany by Beck et al. (2007) allowed some first conclusions to be drawn about buying behavior for a meat product that is higher in price and has undergone a special production process - in this case organic salted meat. The example demonstrates that customers prefer meat products that require some explanation to be placed at the service counter. A reason can be that they will indeed be more likely to be sold from there, as at the service counter the staff is able to promote the product by pointing out its advantages. This also corresponds with the observation that it is mainly consumers who are interested in higher quality and less in price who buy at the service counter (Schulze and Spiller, 2007).

As there is no data available concerning points of sale for high welfare meat, we focus on conventionally produced meat. There is little literature that focuses on the different approaches of how to place processed meat in retail. Nevertheless, some German surveys indicate consumers buy more fresh meat at the service counter than processed meat (LfL, 2013). The percentages of sales for the different channels for fresh and processed meat in Germany are shown in Figure 2 and 3: For fresh meat the supermarkets dominate with $82 \%$, while at least $13 \%$ of the meat is sold at a specialized store; $5 \%$ are sold at further market places like the internet or farm gates. Similar observations can be seen for processed meat, but the share of products sold at the service counter is lot less (25\%) than for fresh meat (45\%) (LfL, 2013).

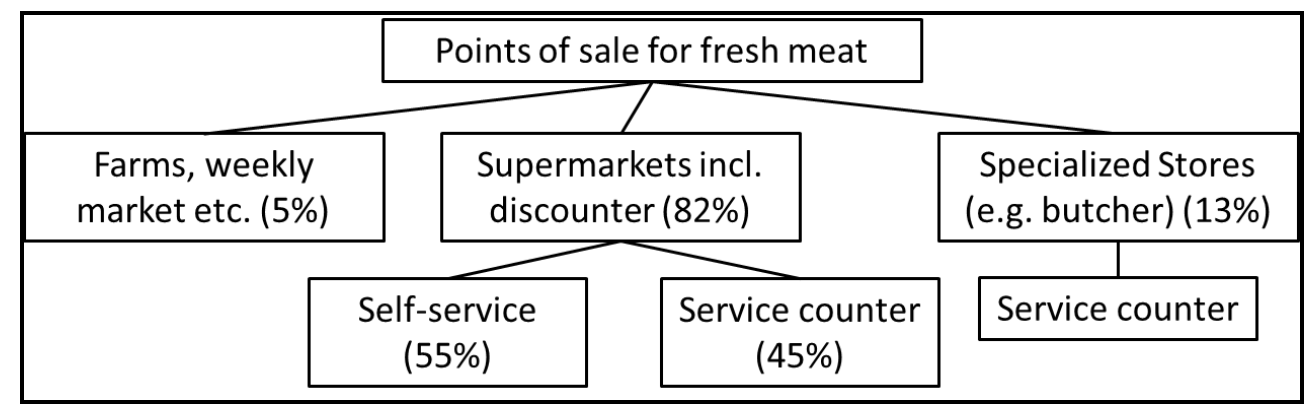

Figure 2. Market shares of the different points of sale for fresh meat in Germany, 2012

Source: Own presentation based on AMI, cited by LfL, 2013 


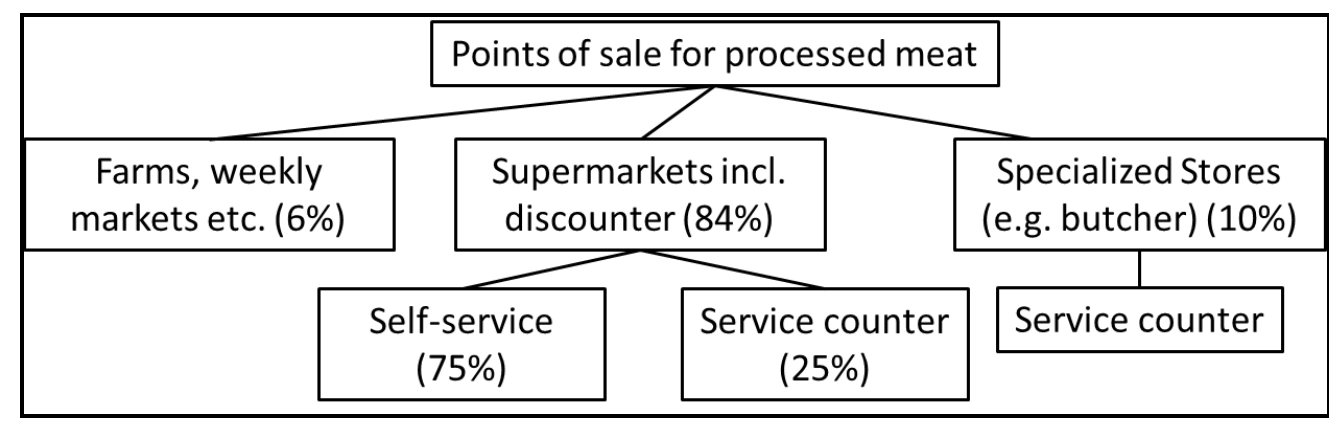

Figure 3. Market shares of the different points of sale for processed meat in Germany, 2012

Source: Own presentation based on AMI, cited by LfL 2013

Due to the lack of research concerning the best placement of high welfare meat in retail the aim of this paper is to examine where high welfare meat and high welfare processed meat should be placed in the supermarket: at the self-service counter or at the service counter. Based on the presented literature, the following hypotheses can be developed:

1. High welfare meat is better placed at the service counter (Beck et al., 2007).

2. For processed meat, the service counter is not as important as it is for fresh meat (LfL, 2013).

In order to test the two hypotheses, an online survey of German meat consumers was conducted. The aim was to get insights into attitudes and buying behaviors regarding labeled meat and labeled processed meat with the focus on animal welfare. Due to the fact that nearly no high welfare meat is available in German retail stores, we use stated preferences to answer these questions.

\section{Methodology}

In August 2012, 642 German household decision makers were asked about their buying behavior regarding meat and processed meat. Besides this, animal welfare and an animal welfare label for meat and processed meat products were also discussed. Responses to statements were given using 7-point $(-3$ to +3$)$ Likert scales. The statements were partly taken from a questionnaire by Schulze et al. (2008a) and partly developed by the authors, as there were no previously tested scales available. The participants were recruited with the help of an online access panel. The sample was subdivided into two groups: 318 respondents were surveyed about their fresh meat consumption, whereas the remaining 324 respondents received analogous questions relating to processed meat.

Quotas were set for age, income and sex of the household decision maker to represent the characteristics of the German population. 

counter?

SPSS Version 20 was used to carry out the data analysis. First, explorative factor analyses were conducted in order to reduce the complexity and number of statements concerning attitudes regarding animal welfare and the attitudes regarding service and self-service counter. Afterwards, four cluster analyses were carried out, two analogues for each sub-sample. Animal welfare cluster analyses were carried out in order to categorize consumers according to their interest in buying animal welfare products using the factors from the factor analyses concerning animal welfare. The other two cluster analyses had the aim of grouping the consumers regarding their preferred point of sale: service counter or self-service counter. For these two analyses, the factors from the factor analyses concerning the preferences for the counters and the single statement "How is your meat shopping split into service counter and self-service counter?" were used. Finally, the result of the two cluster analyses for animal welfare and for counter preferences were combined using cross tabulation analysis, resulting in one cross-tabulation for fresh meat and one for processed meat.

\section{Results of the factor and cluster analyses}

\section{Sample description}

The samples were representative for the quotas that were set to represent age and income characteristics. Additionally, the proportion of male to female household decision makers in the samples represented the distribution in Germany (men: fresh meat sub-sample: $28.9 \%$ [n=291], processed meat sub-sample: $29.6 \%[\mathrm{n}=289]$; women: fresh meat sub-sample: $71.1 \%$, processed meat sub-sample: $70.4 \%$ ). The average age was 46 years in both subsamples. $27.6 \%$ of the participants in the fresh meat sub-sample and $25.1 \%$ in the processed meat sub-sample had a university degree. Table 1 gives more detailed information about the characteristics of both sub-samples. 
Table 1. Characterization of the sub-samples

\begin{tabular}{c|c|c|c}
\hline Variable & $\begin{array}{c}\text { Share fresh meat } \\
\text { Sub-sample }\end{array}$ & $\begin{array}{c}\text { Share processed } \\
\text { meat sub-sample }\end{array}$ & Share Germany $^{\mathbf{1 6}}$ \\
\hline Sample size & 318 & 324 & \\
\hline $\begin{array}{c}\text { Gender of the household de- } \\
\text { cision maker }\end{array}$ & & & \\
\hline Male & $28.9 \%$ & $29.6 \%$ & $30 \%$ \\
\hline Female & $71.1 \%$ & $70.4 \%$ & $70 \%$ \\
\hline Age & & & $33 \%$ \\
\hline 18 to 39 years & $33.7 \%$ & $38.1 \%$ & $35 \%$ \\
\hline 41 to 59 years & $42.6 \%$ & $35.3 \%$ & $32 \%$ \\
\hline 60 years or older & $23.7 \%$ & $26.6 \%$ & $13 \%$ \\
\hline Income & & & $24 \%$ \\
\hline Net income $<900 € /$ month & $11.9 \%$ & $14.8 \%$ & $32 \%$ \\
\hline 901 to $1500 € /$ month & $24.8 \%$ & $22.5 \%$ & $23 \%$ \\
\hline 1501 to $2600 € /$ month & $32.8 \%$ & $30.9 \%$ & $8 \%$ \\
\hline 2601 to $4500 € /$ month & $22.5 \%$ & $23.8 \%$ & - \\
\hline Net income $>4500 € /$ month & $8.0 \%$ & $8.0 \%$ & - \\
\hline Children under 18 years & $75 \%$ & $79 \%$ & - \\
\hline Cohabiting / married & $64 \%$ & $67 \%$ & \\
\hline Education & $27.6 \%$ & $25.1 \%$ & \\
\hline University degree & \multicolumn{2}{|}{} \\
\hline \hline So
\end{tabular}

Source: authors' calculation; Federal Statistical Office (2011)

To ascertain that the panelists buy meat, they were asked about their buying and consumption habits. Vegetarians were screened out. All remaining respondents stated to consume meat at least once a week.

\section{Factor analyses}

To reduce the complexity of the results and to facilitate their interpretation, factor analyses for both sub-samples were carried out. Tables 2 and 3 show the results of the factor analyses sorted according to the survey on fresh meat and the survey on processed meat. The factor analyses for both sub-samples yielded respectively five factors:

- Animal welfare (AW)

- Perceived farm animal welfare situation

- Knowledge of and influence on livestock farming

- Pro service counter (SC)

\footnotetext{
${ }^{16}$ Data is provided for the set quotas.
} 
- Pro self-service counter (SSC)

The factor analyses of both sub-samples have a high Kaiser-Meyer-Olkin criterion $(\mathrm{KMO}=0.864$ for the fresh meat sub-sample and 0.829 for the processed meat sub-sample) (Kaiser, 1974). The Cronbach's alpha values for the fresh meat sub-sample are between 0.611 and 0.942. Overall, three factors have a Cronbach's alpha value $<0.7$ (see Tables 2 and 3). The explained total variance is $70.1 \%$ for the fresh meat sub-sample and $73.7 \%$ for the processed meat sub-sample respectively. Since the factors are comprised of similar constituents, the statements for both sub-samples can be considered to be comparable. The factors can therefore be used for further analogous analyses.

The statements relating to the factors "Perceived farm animal welfare situation" and "Knowledge of and influence on livestock farming" are identical for both sub-samples. Emotional statements dominate the factor "Animal welfare" in the processed meat sub-sample, whereas there are more items referring to buying behavior in the fresh meat sub-sample. The statements for this factor have higher loadings in the fresh meat sub-sample than in the processed meat survey. Two additional quality-based statements are loaded in the factor "Pro service counter", which are not found in the same factor in the fresh meat sub-sample. The factor "Pro self-service counter" focuses on shelf life and practicability for the fresh meat subsample, whereas for the processed meat sub-sample, this factor especially identifies a higher level of information to be important as well as the shelf life. 
Table 2. Results of the factor analysis of the fresh meat sub-sample

\begin{tabular}{|c|c|c|c|}
\hline Factors and the corresponding variables for fresh meat & Mean & Std.dev. & $\begin{array}{l}\text { Factor } \\
\text { loading }\end{array}$ \\
\hline \multicolumn{4}{|l|}{ Animal welfare (Cronbach's alpha: 0.942) } \\
\hline $\begin{array}{l}\text { I would like to have more information about livestock farming when purchasing } \\
\text { meat. }^{1}\end{array}$ & 1.01 & 1.550 & 0.775 \\
\hline I strongly disapprove of livestock farming in big factory farms. ${ }^{1}$ & 1.29 & 1.490 & 0.760 \\
\hline If I knew which meat originates from happy animals I would only buy this meat. ${ }^{1}$ & 1.02 & 1.587 & 0.722 \\
\hline It makes me angry when thinking about how animals are kept in agriculture today. ${ }^{1}$ & 0.94 & 1.691 & 0.706 \\
\hline For me, animal welfare is a selection criterion when buying meat. ${ }^{1}$ & 0.69 & 1.581 & 0.700 \\
\hline I have a problem with meat that originates from factory farms. ${ }^{1,4}$ & 1.02 & 1.558 & 0.683 \\
\hline $\begin{array}{l}\text { I would change my buying behavior if there was meat on offer that was labeled } \\
\text { with an animal welfare label as well as the usual meat on offer in my supermarket. }{ }^{1}\end{array}$ & 0.75 & 1.632 & 0.681 \\
\hline 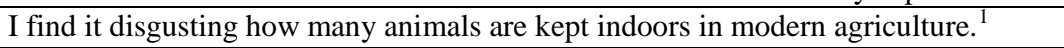 & 1.09 & 1.566 & 0.654 \\
\hline Factory farming is bad for animal welfare. ${ }^{1}$ & 1.62 & 1.433 & 0.648 \\
\hline When doing the shopping, I think about animal welfare. ${ }^{1,4}$ & 0.13 & 1.665 & 0.638 \\
\hline $\begin{array}{l}\text { I am interested in the living conditions of the animals that provide the meat I pur- } \\
\text { chase. } 1\end{array}$ & 0.61 & 1.498 & 0.619 \\
\hline To be honest, I spend a lot of time thinking about animal welfare. ${ }^{1,4}$ & -0.32 & 1.703 & 0.616 \\
\hline $\begin{array}{l}\text { I would like to buy more meat from livestock reared in appropriate conditions, but I } \\
\text { can seldom find any. }\end{array}$ & 0.73 & 1.543 & 0.588 \\
\hline If possible, I buy meat from animals that are treated properly. ${ }^{1}$ & 1.08 & 1.374 & 0.584 \\
\hline In order to buy "high welfare meat" I would also go to the service counter. ${ }^{1}$ & 1.11 & 1.632 & 0.584 \\
\hline \multicolumn{4}{|l|}{ Perceived farm animal welfare situation (Cronbach's alpha: 0.797) } \\
\hline In agriculture, animal welfare has greatly improved in recent years. ${ }^{1}$ & 0.48 & 1.292 & 0.857 \\
\hline In this country, sufficient attention is paid to animal welfare in livestock farming. ${ }^{1}$ & -0.23 & 1.403 & 0.773 \\
\hline \multicolumn{4}{|l|}{ Knowledge of and influence on livestock farming (Cronbach's alpha: 0.611) } \\
\hline $\begin{array}{l}\text { I am knowledgeable about the conditions in which animals are kept in German } \\
\text { agriculture. }{ }^{1}\end{array}$ & 0.48 & 1.350 & 0.789 \\
\hline $\begin{array}{l}\text { Through my buying behavior, I have an influence on the mode of production in } \\
\text { agriculture. }\end{array}$ & 0.59 & 1.636 & 0.643 \\
\hline \multicolumn{4}{|l|}{ Pro service counter (Cronbach's alpha: 0.869) } \\
\hline ...higher quality ${ }^{2}$ & 0.96 & 1.004 & 0.754 \\
\hline$\ldots$ fresher $^{2}$ & 1.00 & 1.108 & 0.732 \\
\hline ...healthier ${ }^{2}$ & 0.53 & 0.894 & 0.687 \\
\hline ...more trustworthy ${ }^{2}$ & 0.93 & 1.094 & 0.678 \\
\hline ....tastier ${ }^{2}$ & 0.86 & 1.050 & 0.659 \\
\hline \multicolumn{4}{|l|}{ Pro self-service counter (Cronbach’s alpha: 0.767 ) } \\
\hline Self-service counter meat has a longer shelf life. ${ }^{3}$ & -0.08 & 1.434 & 0.833 \\
\hline ...more likely to have a longer shelf life. ${ }^{2}$ & -0.30 & 1.178 & 0.776 \\
\hline It's good that self-service counter meat has a longer shelf life. $^{1}$ & 0.10 & 1.426 & 0.545 \\
\hline The packaging of self-service counter meat is more practical. ${ }^{3}$ & 0.40 & 1.509 & 0.521 \\
\hline
\end{tabular}

"Scale from -3 "Not correct at all" to +3 "Fully correct"

${ }^{2}$ Scale from -3 "Self-service counter meat is much..." to 0 "Indifferent" to +3 "Service counter meat is much..."

${ }^{3}$ Scale from -3 "I totally disagree" to +3 "I totally agree"

${ }^{4}$ Statement was recoded

$\mathrm{n}=324$

Source: authors' calculation 
Table 3. Results of the factor analysis of the processed meat sub-sample

\begin{tabular}{|c|c|c|c|}
\hline Factors and the corresponding variables for processed meat & Mean & Std.dev. & $\begin{array}{l}\text { Factor } \\
\text { loading }\end{array}$ \\
\hline \multicolumn{4}{|l|}{ Animal welfare (Cronbach's alpha: 0.939) } \\
\hline It makes me angry when thinking about how animals are kept in agriculture today. ${ }^{1}$ & 0.73 & 1.546 & 0.822 \\
\hline $\begin{array}{l}\text { I find it sad that nowadays so many animals are kept in the narrowest of spaces so } \\
\text { that we can buy cheap meat. }{ }^{1}\end{array}$ & 1.42 & 1.404 & 0.809 \\
\hline Factory farming is bad for animal welfare. ${ }^{1}$ & 1.42 & 1.441 & 0.792 \\
\hline I strongly disapprove of livestock farming in big factory farms. ${ }^{1}$ & 1.01 & 1.596 & 0.784 \\
\hline I find it disgusting how many animals are kept indoors in modern agriculture. ${ }^{1}$ & 0.93 & 1.538 & 0.749 \\
\hline For me, animal welfare is a selection criterion when buying meat. ${ }^{1}$ & 0.36 & 1.571 & 0.725 \\
\hline $\begin{array}{l}\text { If I knew which processed meat originates from happy animals I would only buy } \\
\text { this processed meat. }{ }^{1}\end{array}$ & 0.72 & 1.616 & 0.714 \\
\hline $\begin{array}{l}\text { In order to buy "high welfare processed meat" I would also go to the service coun- } \\
\text { ter. }{ }^{1}\end{array}$ & 0.93 & 1.743 & 0.640 \\
\hline I have a problem with processed meat that originates from factory farms. ${ }^{1,4}$ & 0.72 & 1.487 & 0.609 \\
\hline $\begin{array}{l}\text { I would like to have more information about livestock farming when purchasing } \\
\text { processed meat. }\end{array}$ & 0.51 & 1.616 & 0.600 \\
\hline $\begin{array}{l}\text { I would change my buying behavior if there was processed meat on offer that was } \\
\text { labeled with an animal welfare label as well as the usual processed meat on offer in } \\
\text { my supermarket. }{ }^{1}\end{array}$ & 0.25 & 1.597 & 0.597 \\
\hline To be honest, I spend a lot of time thinking about animal welfare. ${ }^{1,4}$ & -0.28 & 1.685 & 0.558 \\
\hline \multicolumn{4}{|l|}{ Perceived farm animal welfare situation (Cronbach's alpha: 0.668 ) } \\
\hline In agriculture, animal welfare has greatly improved in recent years. ${ }^{1}$ & 0.49 & 1.142 & 0.8672 \\
\hline In this country, sufficient attention is paid to animal welfare in livestock farming. ${ }^{1}$ & -0.21 & 1.278 & 0.8672 \\
\hline \multicolumn{4}{|l|}{ Knowledge of and influence on livestock farming (Cronbach's alpha: 0.620) } \\
\hline $\begin{array}{l}\text { I am knowledgeable about the conditions in which animals are kept in German } \\
\text { agriculture. }{ }^{1}\end{array}$ & 0.45 & 1.369 & 0.8523 \\
\hline $\begin{array}{l}\text { Through my buying behavior, I have an influence on the mode of production in } \\
\text { agriculture. }\end{array}$ & 0.73 & 1.546 & 0.8523 \\
\hline \multicolumn{4}{|l|}{ Pro service counter (Cronbach's alpha: 0.858$)$} \\
\hline ...higher quality ${ }^{2}$ & 0.85 & 1.072 & 0.794 \\
\hline$\ldots$ fresher $^{2}$ & 0.98 & 1.254 & 0.788 \\
\hline ...more trustworthy ${ }^{2}$ & 0.58 & 1.227 & 0.732 \\
\hline$\ldots$ tastier $^{2}$ & 0.82 & 1.152 & 0.720 \\
\hline $\begin{array}{l}\text { Processed meat from the service counter looks more delicious than processed meat } \\
\text { from the self-service counter. }\end{array}$ & 0.34 & 1.324 & 0.666 \\
\hline$\ldots$ healthier ${ }^{2}$ & 0.33 & 0.908 & 0.649 \\
\hline Processed meat from the self-service counter often contains flavor enhancer. ${ }^{3}$ & 0.66 & 1.254 & 0.606 \\
\hline \multicolumn{4}{|l|}{ Pro self-service counter (Cronbach's alpha: 0.710 ) } \\
\hline I can examine self-service counter processed meat products more carefully. ${ }^{3}$ & 0.50 & 1.298 & 0.824 \\
\hline There is useful information on the packaging of processed meat products. ${ }^{3}$ & 0.52 & 1.286 & 0.770 \\
\hline Self-service counter processed meat products have a longer shelf life. ${ }^{3}$ & 0.58 & 1.429 & 0.649 \\
\hline \multicolumn{4}{|l|}{ KMO $($ Kaiser-Meyer-Olkin $)=0.829 ;$ explained total variance $=73.70 \%$} \\
\hline $\begin{array}{l}{ }^{1} \text { Scale from }-3 \text { "Not correct at all" to }+3 \text { "Fully correct" } \\
{ }^{2} \text { Scale from }-3 \text { "Self-service counter meat is much..." to } 0 \text { "Indifferent" to }+3 \text { "Servic } \\
{ }^{3} \text { Scale from }-3 \text { "I totally disagree" to }+3 \text { "I totally agree" } \\
{ }^{4} \text { Statement was recoded } \\
n=318\end{array}$ & ounter $\mathrm{m}$ & is much..." & \\
\hline
\end{tabular}

Source: authors' calculation

In the next step, two cluster analyses were conducted for each sub-sample, one for "Attitudes towards animal welfare" and one for "Attitudes towards the preference for service counter or self-service counter". The results of these analyses are presented separately. 


\section{Cluster analysis "Attitudes towards animal welfare"}

As suggested by Schulze et al. (2008b), the cluster analysis was conducted in several steps. First, the single linkage method was used. In each data set, three outliers were identified and deleted. Next, the optimal number of clusters was determined using Ward's hierarchical clustering method. Based on the results of a scree test, a dendrogram and plausibility considerations, a three cluster solution was chosen. The solution was refined by applying the K-means algorithm. $8.25 \%$ of the fresh meat buyers and $12.97 \%$ of the processed meat buyers were reassigned. The F-values for all factors are significant at the $1 \%$ level. For eta, there was an average value of 0.716 ( 0.73 for the animal welfare clusters of the processed meat subsample) and for eta-squared 0.51 ( 0.53 for the processed meat sub-sample). Hence, $51.47 \%$ and $53.33 \%$ of the variance can be explained by differences between the clusters.

Next, a discriminant analysis was conducted. It showed that $98.1 \%$ of cases in the fresh meat sub-sample and $96.2 \%$ in the processed meat sub-sample were correctly allocated. Analysis of variance (ANOVA) was used to describe the clusters. Finally, post hoc tests were carried out in order to identify significant differences between the means of the clusters (Everitt, 1998). The results of these last analyses are presented in Tables A and B in the appendix. They show the results of the cluster analyses for "Attitudes towards animal welfare" including the variables describing the factors. For simplification, these clusters are named "AW clusters". The first cluster is the largest. In the fresh meat sub-sample, 133 cases (42.6\%) are allocated to this cluster, while 148 cases $(53.1 \%)$ are allocated in the processed meat subsample. Animal welfare is not an important issue for this group $\left(\mu_{\mathrm{FM}}=-0.84 ; \mu_{\mathrm{PM}}=-0.69\right)^{17}$. For the factor "Perceived farm animal welfare situation" there is only a slight positive tendency $\left(\mu_{\mathrm{FM}}=0.13 ; \mu_{\mathrm{PM}}=0.04\right)$. The factor "Knowledge of and influence on livestock farming" is assigned the most negative attitude $\left(\mu_{\mathrm{FM}}=-0.73 ; \mu_{\mathrm{PM}}=-0.55\right)$. Hence, this cluster can be named the "AW indifferent".

The second cluster contains 88 cases $(28.2 \%)$ for the fresh meat sub-sample and 64 cases $(22.9 \%)$ for processed meat sub-sample, making it the smallest cluster. For the factor "Animal welfare" there is a general attitude of agreement $\left(\mu_{\mathrm{FM}}=0.39 ; \mu_{\mathrm{PM}}=0.42\right)$. The factor "Perceived farm animal welfare situation" achieves the highest factor mean values for both sub-samples $\left(\mu_{\mathrm{FM}}=0.74 ; \mu_{\mathrm{PM}}=0.91\right)$. The respondents in this cluster are most likely to be of the opinion that the level of animal welfare in German agriculture is high and that it is given

\footnotetext{
${ }^{17} \mathrm{FM}=$ fresh meat survey; $\mathrm{PM}=$ processed meat survey
} 
enough attention. The members of this cluster also think they have good knowledge about livestock farming in Germany which results in the highest factor mean value in both subsamples $\left(\mu_{\mathrm{FM}}=0.85 ; \mu_{\mathrm{PM}}=0.8\right)$. Thus, this cluster can be characterized as the "AW pragmatists".

The last cluster contains 91 (29.2\%) cases for the fresh meat sub-sample and 67 (24\%) cases for the processed meat sub-sample. It contains the highest mean values for "Animal welfare" with $\mu_{\mathrm{FM}}=0.88$ for the fresh meat sub-sample and $\mu_{\mathrm{PM}}=1.07$ for the processed meat subsample. The cluster members regard "Perceived farm animal welfare situation" with skepticism, which results in the most negative factor mean values $\left(\mu_{\mathrm{FM}}=-0.98 ; \mu_{\mathrm{PM}}=-0.88\right)$. The factor "Knowledge of and influence on the livestock farming" is less pronounced than in cluster two $\left(\mu_{\mathrm{FM}}=0.29 ; \mu_{\mathrm{PM}}=0.48\right)$ and the statements relating to "Perceived farm animal welfare situation" have the most negative factor mean values. Hence, this cluster can be named "AW enthusiasts".

\section{Cluster analysis for "Attitudes towards the preference for service counter or self-service counter"}

In addition to the cluster analyses for "Attitudes towards animal welfare", the respondents were divided into groups in order to find out if they prefer buying meat and processed meat at the self-service counter or at the service counter. The approach was analogous to the cluster analyses described previously. By means of the single-linkage method, three outliers were deleted from the fresh meat sub-sample and twelve from the processed meat sub-sample. A scree test, a dendrogram and plausibility considerations, together with Ward's method, yielded an optimal cluster number of three for each analysis. K-means cluster analysis led to a regrouping of $7.39 \%$ of all cases in the fresh meat sub-sample, while in the processed meat subsample one case was regrouped. The F-values of all factors and the single statement "How is your meat shopping split into service counter and self-service counter?" are highly significant at the $1 \%$-level. An eta value of 0.66 ( 0.63 for the processed meat sub-sample) was calculated. Based on eta-squared, $47.5 \%$ (52.63\% for the processed meat sub-sample) of the cluster factors and the single statement can be explained by differences between the clusters. A discriminant analysis shows that $96.5 \%$ or $98.5 \%$ of all cases are correctly allocated. An ANOVA and post-hoc tests were also conducted analogously to the first two cluster analyses. Tables 6 and 7 show the results of these analyses. 
Table 6. Food counter clusters for the fresh meat sub-sample

\begin{tabular}{|c|c|c|c|}
\hline & $\begin{array}{l}\text { Cluster } \mathbf{A}_{\mathrm{FM}} \text { : } \\
\text { SSC fans }\end{array}$ & $\begin{array}{c}\text { Cluster B } \text { FM: }_{\text {Combiners }} \\
\text { Comb }\end{array}$ & $\begin{array}{c}\text { Cluster } \mathrm{C}_{\mathrm{FM}} \text { : } \\
\mathrm{SC} \text { fans }\end{array}$ \\
\hline Cluster size (n) & 69 & 147 & 68 \\
\hline in $\%$ & 24.3 & 51.8 & 23.9 \\
\hline Factor $1_{\mathrm{FM}}$ : Pro service counter $* * *$ & $\begin{array}{l}-0.70^{b c} \\
(0.624) \\
\end{array}$ & $\begin{array}{c}-0.06^{\mathrm{ac}} \\
(0.78)\end{array}$ & $\begin{array}{c}0.91^{\text {ab }} \\
(0.999) \\
\end{array}$ \\
\hline ...higher quality ${ }^{1} * * *$ & $\begin{array}{c}0.46^{\mathrm{bc}} \\
(0.759)\end{array}$ & $\begin{array}{l}0.93^{\mathrm{ac}} \\
(1.05)\end{array}$ & $\begin{array}{c}1.65^{\mathrm{ab}} \\
(1.062)\end{array}$ \\
\hline ...fresher ${ }^{1} * * *$ & $\begin{array}{l}0.41^{\mathrm{bc}} \\
(0.754)\end{array}$ & $\begin{array}{l}0.93^{\mathrm{ac}} \\
(1.05)\end{array}$ & $\begin{array}{l}1.85^{a b} \\
(0.966)\end{array}$ \\
\hline ...healthier ${ }^{1} * * *$ & $\begin{array}{c}0.19^{\mathrm{bc}} \\
(0.493)\end{array}$ & $\begin{array}{l}0.41^{\mathrm{ac}} \\
(0.73)\end{array}$ & $\begin{array}{c}1.16^{\mathrm{ab}} \\
(1.192)\end{array}$ \\
\hline ...more trustworthy ${ }^{1} * * *$ & $\begin{array}{l}0.25^{\mathrm{bc}} \\
(0.695)\end{array}$ & $\begin{array}{l}0.87^{\mathrm{ac}} \\
(0.97)\end{array}$ & $\begin{array}{l}1.84^{\mathrm{ab}} \\
(1.002)\end{array}$ \\
\hline ...tastier ${ }^{1} * * *$ & $\begin{array}{c}0.30^{\mathrm{bc}} \\
(0.692)\end{array}$ & $\begin{array}{l}0.80^{\mathrm{ac}} \\
(0.96)\end{array}$ & $\begin{array}{l}1.59^{\mathrm{ab}} \\
(1.054)\end{array}$ \\
\hline Factor $2_{\mathrm{FM}}$ : Pro self-service counter*** & $\begin{array}{c}0.55^{b c} \\
(0.905) \\
\end{array}$ & $\begin{array}{l}0.14^{\mathrm{ac}} \\
(0.78) \\
\end{array}$ & $\begin{array}{l}-0.80^{\mathrm{ab}} \\
(1.007) \\
\end{array}$ \\
\hline SSC meat has a longer shelf life. ${ }^{2} * * *$ & $\begin{array}{c}0.23^{\mathrm{c}} \\
(1.487)\end{array}$ & $\begin{array}{l}0.16^{\mathrm{c}} \\
(1.26)\end{array}$ & $\begin{array}{l}-0.85^{\mathrm{ab}} \\
(1.448)\end{array}$ \\
\hline ...is more likely to have a longer shelf life $\mathrm{e}^{1 * * *}$ & $\begin{array}{c}0.97^{\mathrm{bc}} \\
(0.939)\end{array}$ & $\begin{array}{l}0.52^{\mathrm{ac}} \\
(1.11)\end{array}$ & $\begin{array}{l}-0.16^{\mathrm{ab}} \\
(1.300)\end{array}$ \\
\hline It's good that self-service counter meat has a longer shelf life. ${ }^{3} * * *$ & $\begin{array}{l}0.86^{\mathrm{bc}} \\
(1.309)\end{array}$ & $\begin{array}{c}0.26^{\mathrm{ac}} \\
(1.135)\end{array}$ & $\begin{array}{l}-0.99^{\mathrm{ab}} \\
(1.419)\end{array}$ \\
\hline The packaging of self-service counter meat is more practical. ${ }^{2} * * *$ & $\begin{array}{l}1.17^{\mathrm{bc}} \\
(1.306)\end{array}$ & $\begin{array}{l}0.52^{\mathrm{ac}} \\
(1.32)\end{array}$ & $\begin{array}{l}-0.60^{\mathrm{ab}} \\
(1.517)\end{array}$ \\
\hline $\begin{array}{l}\text { How is your meat shopping split into service counter and self- } \\
\text { service counter? }{ }^{4} * *\end{array}$ & $\begin{array}{l}-1.96^{\mathrm{bc}} \\
(0.716)\end{array}$ & $\begin{array}{l}0.10^{\mathrm{ac}} \\
(0.565)\end{array}$ & $\begin{array}{r}2.25^{\mathrm{ab}} \\
(0.608)\end{array}$ \\
\hline
\end{tabular}

Significance level: $* * *=p \leq 0.001, * *=p \leq 0.01, *=p \leq 0.05$; mean value; (standard deviation); letters mark a significant difference between groups (Tamhane's post hoc test T2 at a significance level 0.05)

${ }^{1}$ Scale from -3 "Not correct at all" to +3 "Fully correct"

${ }^{2}$ Scale from -3 "I totally disagree" to +3 "I totally agree"

${ }^{3}$ Scale from -2 "No, never" to +2 "Yes, definitely"

${ }^{4}$ Scale from -3 "Always self-service counter" to +3 "Always service counter"

$\mathrm{SSC}=$ Self-service counter

$\mathrm{SC}=$ Service counter

$\mathrm{FM}=$ Fresh meat

Source: authors' calculation 

counter?

Table 7. Food counter clusters for the processed meat sub-sample

\begin{tabular}{|c|c|c|c|}
\hline & $\begin{array}{l}\text { Cluster } A_{P M}: \\
\text { SSC fans }\end{array}$ & $\begin{array}{c}\text { Cluster B } \text { BM }_{\text {PM }} \\
\text { Combiners }\end{array}$ & $\begin{array}{c}\text { Cluster } \mathbf{C}_{\mathbf{P M}} \\
\text { SC fans }\end{array}$ \\
\hline $\begin{array}{l}\text { Cluster size (n) } \\
\text { in } \%\end{array}$ & $\begin{array}{l}108 \\
41.1\end{array}$ & $\begin{array}{c}96 \\
36.5\end{array}$ & $\begin{array}{c}59 \\
22.4\end{array}$ \\
\hline Factor $1_{\mathrm{PM}}$ : Pro service counter*** & $\begin{array}{l}-0.40^{\mathrm{bc}} \\
(0.607)\end{array}$ & $\begin{array}{l}-0.16^{\mathrm{ac}} \\
(0.702) \\
\end{array}$ & $\begin{array}{c}1.11^{\mathrm{ab}} \\
(0.739)\end{array}$ \\
\hline ...higher quality ${ }^{1} * * *$ & $\begin{array}{l}0.46^{\mathrm{bc}} \\
(0.759)\end{array}$ & $\begin{array}{l}0.93^{\mathrm{ac}} \\
(1.05)\end{array}$ & $\begin{array}{l}1.65^{\mathrm{ab}} \\
(1.062)\end{array}$ \\
\hline ...fresher ${ }^{1} * * *$ & $\begin{array}{l}0.41^{\text {bc }} \\
(0.754)\end{array}$ & $\begin{array}{l}0.93^{\mathrm{ac}} \\
(1.05)\end{array}$ & $\begin{array}{l}1.85^{\mathrm{ab}} \\
(0,966)\end{array}$ \\
\hline ...healthier ${ }^{1} * * *$ & $\begin{array}{c}0.19^{\mathrm{bc}} \\
(0.493)\end{array}$ & $\begin{array}{l}0.41^{\mathrm{ac}} \\
(0.73)\end{array}$ & $\begin{array}{l}1.16^{\mathrm{ab}} \\
(1.192)\end{array}$ \\
\hline ...more trustworthy ${ }^{1} * * *$ & $\begin{array}{l}0.25^{\text {bc }} \\
(0.695)\end{array}$ & $\begin{array}{l}0.87^{\text {ac }} \\
(0.97)\end{array}$ & $\begin{array}{c}1.84 \\
(1.002)\end{array}$ \\
\hline$\ldots \operatorname{tastier}^{1 * * *}$ & $\begin{array}{l}0.30^{\mathrm{bc}} \\
(0.692)\end{array}$ & $\begin{array}{l}0.80^{\mathrm{ac}} \\
(0.96)\end{array}$ & $\begin{array}{l}1.59^{\mathrm{ab}} \\
(1.054)\end{array}$ \\
\hline Factor $2_{\mathrm{PM}}$ : Pro self-service counter*** & $\begin{array}{c}0.37^{\text {bc }} \\
(0.819)\end{array}$ & $\begin{array}{c}0.03^{\text {ac }} \\
(0.866)\end{array}$ & $\begin{array}{l}-0.61^{\mathrm{ab}} \\
(0.855)\end{array}$ \\
\hline $\begin{array}{l}\text { I can examine self-service counter processed meat products more } \\
\text { carefully. }{ }^{2 * * *}\end{array}$ & $\begin{array}{c}0.99^{\mathrm{bc}} \\
(1.106)\end{array}$ & $\begin{array}{c}0.46^{\mathrm{ac}} \\
(1.187)\end{array}$ & $\begin{array}{l}-0.24^{\mathrm{ab}} \\
(1.150)\end{array}$ \\
\hline $\begin{array}{l}\text { There is useful information on the packaging of processed meat } \\
\text { products. }{ }^{2 * * *}\end{array}$ & $\begin{array}{l}0.79^{c} \\
(1.144)\end{array}$ & $\begin{array}{c}0.65^{\mathrm{c}} \\
(1.170)\end{array}$ & $\begin{array}{l}-0.12^{\mathrm{ab}} \\
(1.205)\end{array}$ \\
\hline $\begin{array}{l}\text { Self-service counter processed meat products have a longer shelf } \\
\text { life. }\end{array}$ & $\begin{array}{l}1.12^{\mathrm{bc}} \\
(1.125)\end{array}$ & $\begin{array}{c}0.70^{\mathrm{a}} \\
(1.282)\end{array}$ & $\begin{array}{c}0.19^{\mathrm{a}} \\
(1.420)\end{array}$ \\
\hline $\begin{array}{l}\text { How is your meat shopping split into service counter and self- } \\
\text { service counter? }\end{array}$ & $\begin{array}{l}-1.92^{\text {bc }} \\
(0.699)\end{array}$ & $\begin{array}{c}0.13^{\text {ac }} \\
(0.548)\end{array}$ & $\begin{array}{l}1.73^{\text {ab }} \\
(0.691)\end{array}$ \\
\hline
\end{tabular}

Significance level: $* * *=p \leq 0.001, * *=p \leq 0.01, *=p \leq 0.05$; mean value; (standard deviation); letters mark a significant difference between groups (Tamhane's post hoc test T2 at a significance level 0.05 )

${ }^{1}$ Scale from -3 "Not correct at all" to +3 "Fully correct"

${ }^{2}$ Scale from -3 "I totally disagree" to +3 "I totally agree"

${ }^{3}$ Scale from -3 "Always self-service counter" to +3 "Always service counter"

$\mathrm{SSC}=$ Self-service counter

$\mathrm{SC}=$ Service counter

$\mathrm{PM}=$ Processed meat

Source: authors' calculation

The three clusters differ in the factors "Pro service counter" and "Pro self-service counter" as well as the single statement "How is your meat shopping split into service counter and selfservice counter?" (7-point Likert scale from 3 "Always service counter" to 0 "Equally divided" to +3 "Always self-service counter", henceforth named "Distribution SSC / SC") differed significantly in their mean values. The first cluster has a significantly negative mean value for the factor "Pro service counter" $\left(\mu_{\mathrm{FM}}=-0.7 ; \mu_{\mathrm{PM}}=-0.4\right)$ and the single statement "Distribution SSC / SC" $\left(\mu_{\mathrm{FM}}=-1.96 ; \mu_{\mathrm{PM}}=-1.92\right)$. For the factor "Pro self-service counter" this cluster has positive values $\left(\mu_{\mathrm{F}}=0.55 ; \mu_{\mathrm{W}}=0.37\right)$ for both fresh and processed meat sub-samples. Hence, the first cluster can be characterized as "Self-service counter (SSC) fans".

The second cluster shows no clear tendency towards a positive or negative pronouncement for both these factors as well as for the single statement ("Pro service counter": $\mu_{\mathrm{FM}}=-0.06 ; \mu_{\mathrm{PM}}$ 
$=-0.16$; "Pro self-service counter": $\mu_{\mathrm{FM}}=0.14 ; \mu_{\mathrm{PM}}=-0.03$; "Distribution SSC / SC": $\left.\mu_{\mathrm{FM}}=0.1 ; \mu_{\mathrm{PM}}=0.13\right)$. Thus, this cluster is named "Combiners".

The third cluster shows a tendency towards the service counter for both fresh and processed meat sub-samples. The statement "Distribution SSC / SC" reveals that the respondents preferably buy at the service counter $\left(\mu_{\mathrm{FM}}=2.25 ; \mu_{\mathrm{PM}}=1.73\right)$. The third cluster also has the most negative factor mean values for the factor "Pro self-service counter" $\left(\mu_{\mathrm{FM}}=-0.80 ; \mu_{\mathrm{PM}}=-\right.$ 0.61), while having the most positive factor mean values with the factor "Pro service counter" $\left(\mu_{\mathrm{FM}}=0.91 ; \mu_{\mathrm{PM}}=1.11\right)$. Therefore, this cluster will be described as "Service counter (SC) fans".

\section{Cross tabulation analyses for fresh and processed meat consumers}

Cross tabulations were calculated in order to compare the results of the "Attitudes towards animal welfare" and the "Attitudes towards the preference for service counter or self-service counter". The aim of the cross tabulation analysis was to detect at which counter potential high welfare meat buyers can be targeted more effectively. This was done twice: once for the fresh meat sub-sample and once for the processed meat sub-sample. The resulting relationships between the animal welfare clusters and the food counter clusters are shown in Tables 8 and 9.

Table 8. Results of cross tabulation for the animal welfare and counter clusters (fresh meat)

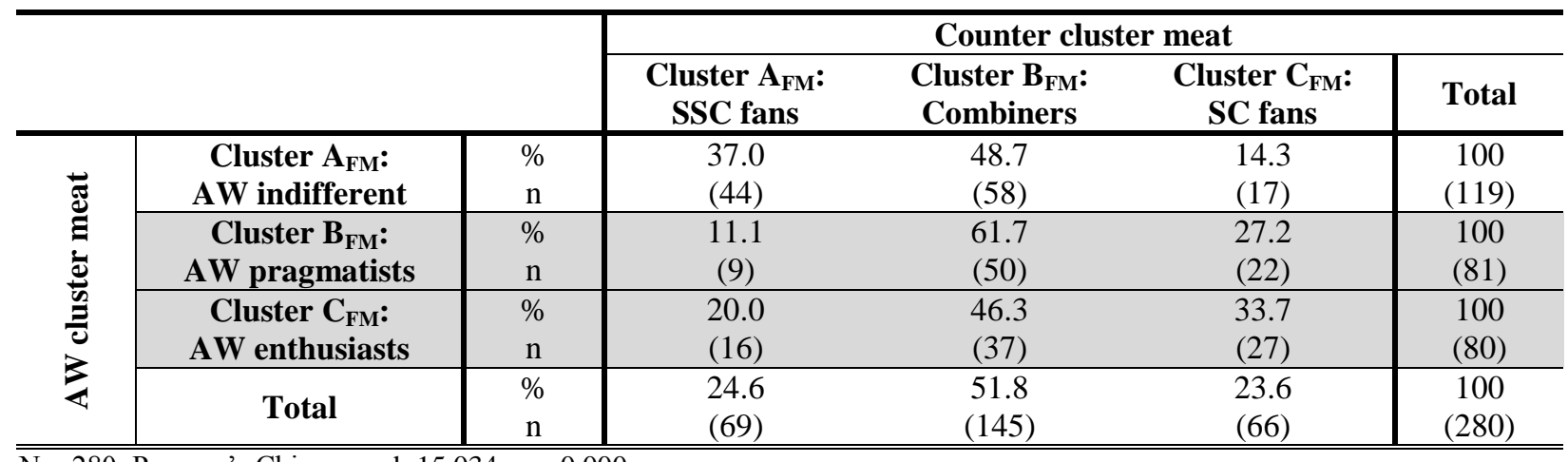

N = 280; Pearson's Chi-squared: 15.034; $\mathrm{p}=0.000$

$\mathrm{AW}=$ animal welfare; $_{\mathrm{FM}}=$ fresh meat; target groups are highlighted in grey

Source: authors' calculation

For the fresh meat sub-sample, the target group consists of the AW pragmatists and AW enthusiasts (highlighted in grey in Table 8). This group has 161 consumers in total (81 plus 80) which is $57.5 \%$ of the total number of 280 consumers. This share is considered as $100 \%$ in the following calculations. Thus, averages are calculated for each counter cluster. On average, $15.5 \%$ (calculation example: $11.1 \%$ of the AW pragmatists plus $20.0 \%$ of the AW enthusiast divided by two) of the AW pragmatists and AW enthusiasts preferably buy meat from the 

counter?

self-service counter. $54.0 \%$ buy at both the service counter and self-service counter on average. $30.5 \%$ buy their meat at the service counter. The results are significant on the $0.1 \%$ level $(\mathrm{p}=0.000)$.

Table 9. Results of cross tabulation for the animal welfare and counter clusters (processed meat)

\begin{tabular}{|c|c|c|c|c|c|c|}
\hline & & & & unter cluster & sed meat & \\
\hline & & & $\begin{array}{l}\text { Cluster } A_{P M}: \\
\text { SSC fans }\end{array}$ & $\begin{array}{c}\text { Cluster B } \text { BM }_{\text {PM }} \\
\text { Combiners }\end{array}$ & $\begin{array}{c}\text { Cluster } C_{P M}: \\
\text { SC fans }\end{array}$ & Total \\
\hline & $\begin{array}{c}\text { Cluster } A_{P M}: \\
\text { AW indifferent }\end{array}$ & $\begin{array}{l}\% \\
\mathrm{n}\end{array}$ & $\begin{array}{l}48.0 \\
(60)\end{array}$ & $\begin{array}{l}35.2 \\
(44)\end{array}$ & $\begin{array}{l}16.8 \\
(21)\end{array}$ & $\begin{array}{c}100 \\
(125)\end{array}$ \\
\hline పี & $\begin{array}{c}\text { Cluster } B_{\mathrm{PM}} \text { : } \\
\text { AW pragmatists }\end{array}$ & $\begin{array}{l}\% \\
\mathrm{n}\end{array}$ & $\begin{array}{l}29.8 \\
(17)\end{array}$ & $\begin{array}{l}40.4 \\
(23)\end{array}$ & $\begin{array}{l}29.8 \\
(17)\end{array}$ & $\begin{array}{l}100 \\
(57)\end{array}$ \\
\hline 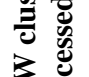 & $\begin{array}{c}\text { Cluster } \mathbf{C}_{\mathbf{P M}} \text { : } \\
\text { AW enthusiasts }\end{array}$ & $\begin{array}{l}\% \\
\mathrm{n}\end{array}$ & $\begin{array}{l}41.1 \\
(23) \\
\end{array}$ & $\begin{array}{l}33.9 \\
(19) \\
\end{array}$ & $\begin{array}{l}25.0 \\
(14) \\
\end{array}$ & $\begin{array}{l}100 \\
(56) \\
\end{array}$ \\
\hline & Total & $\begin{array}{l}\% \\
\mathrm{n}\end{array}$ & $\begin{array}{l}42.0 \\
(100)\end{array}$ & $\begin{array}{l}36.2 \\
(86)\end{array}$ & $\begin{array}{l}21.8 \\
(52)\end{array}$ & $\begin{array}{c}100 \\
(238)\end{array}$ \\
\hline
\end{tabular}

$\mathrm{N}=$ 238; Pearson's Chi-squared: $3.669 ; \mathrm{p}=0.144$

$\mathrm{AW}=$ animal welfare; ${ }_{\mathrm{PM}}=$ processed meat; target groups are highlighted in grey

Source: authors' calculation

For the processed meat sub-sample, the results are less clear and cannot be considered significant $(\mathrm{p}=0.144)$. Again, the AW pragmatists together with the AW enthusiasts form the target group for high welfare processed meat (highlighted in grey in Table 9) with 113 consumers (57 plus 56) which is $47.5 \%$ of the total number of 238 consumers. This target group is considered as $100 \%$ hereafter. On average, $35.5 \%$ of the AW pragmatists and AW enthusiasts prefer the self-service counter (calculation example: $29.8 \%$ plus $41.1 \%$ divided by two). $37.1 \%$ buy at both the self-service and the service counter. $27.4 \%$ opt mostly for the service counter.

\section{Discussion}

\section{Discussion of the animal welfare clusters}

This present study identifies strong differences in the attitudes of consumers that help classifying the target group for high welfare fresh and processed meat: We find that consumers can be clustered into sub-groups which we describe as "AW indifferent", "AW pragmatists" and the "AW enthusiasts". The AW indifferent account for $42.6 \%$ in the fresh meat sub-sample and for $53.1 \%$ in the processed meat sub-sample. The AW indifferent are less suitable as target group for high welfare meat as they show a negative attitude towards animal welfare $\left(\mu_{\mathrm{FM}}\right.$ $\left.=-0.84 ; \mu_{\mathrm{PM}}=-0.69\right)$. On contrary, the AW pragmatists and the AW enthusiasts are very suitable as target group for high welfare meat. In both sub-samples, the cluster have high factor mean values for the attitude towards animal welfare $\left(\mu_{\mathrm{FM}}=0.39\right.$ and $\mu_{\mathrm{PM}}=0.42$ for the AW 
pragmatists; $\mu_{\mathrm{FM}}=0.88$ and $\mu_{\mathrm{PM}}=1.07$ for the AW enthusiasts). Nevertheless, both clusters in both sub-samples differ in their perceived farm animal welfare situation. The AW perceive the farm animal welfare positively $\left(\mu_{\mathrm{FM}}=0.74 ; \mu_{\mathrm{PM}}=0.91\right)$ whereas the AW enthusiast have a negative perception $\left(\mu_{\mathrm{FM}}=-0.98 ; \mu_{\mathrm{PM}}=-0.88\right)$. The AW pragmatists and the AW enthusiasts have in common that they have positive factor mean values for the knowledge and influence on livestock farming in both sub-samples welfare $\left(\mu_{\mathrm{FM}}=0.85\right.$ and $\mu_{\mathrm{PM}}=0.80$ for the AW pragmatists; $\mu_{\mathrm{FM}}=0.29$ and $\mu_{\mathrm{PM}}=0.48$ for the AW enthusiasts) with respect to the $\mathrm{AW}$ indifferent who have negative factor mean values in both sub-samples $\left(\mu_{\mathrm{FM}}=-0.73 ; \mu_{\mathrm{PM}}=-\right.$ 0.55). Due to these results, the AW pragmatists and the AW enthusiast are the target group for high welfare processed and fresh meat.

\section{Discussion of the counter clusters}

The cluster analyses for the food counter show strong differences concerning consumer attitudes as well. We find in both sub-samples the "SSC fans", the "Combiners" and the "SC fans". The SSC fans have a share of $24.3 \%$ in the fresh meat sub-sample and $41.1 \%$ in the processed meat sub-sample. In both sub-samples, the SSC fans prefer the self-service counter $\left(\mu_{\mathrm{FM}}=0.55 ; \mu_{\mathrm{PM}}=0.37\right)$ most strongly compared to the other clusters, show the most negative factor mean values for the service counter $\left(\mu_{\mathrm{FM}}=-0.70 ; \mu_{\mathrm{PM}}=-0.40\right)$ and do their shopping at the self-service counter $\left(\mu_{\mathrm{FM}}=-1.96\right.$ and $\mu_{\mathrm{PM}}=-1.92$ for the cluster forming statement "How is your meat shopping split into service counter and self-service counter?"). Results fit to the market data presented in Figures 2 and 3. The Combiners account for the biggest group with $51.8 \%$ (fresh meat sub-sample) and $36.5 \%$ (processed meat sub-sample) do not reveal a specific preference for any of the counters $\left(\mu_{\mathrm{FM}}=-0.06\right.$ and $\mu_{\mathrm{PM}}=-0.16$ for factor 1 ; $\mu_{\mathrm{FM}}=0.14$ and $\mu_{\mathrm{PM}}=0.03$ for factor $2 ; \mu_{\mathrm{FM}}=0.10$ and $\mu_{\mathrm{PM}}=0.13$ for the cluster forming statement "How is your meat shopping split into service counter and self-service counter?"). The SC fans have the opposite results compared to the SSC fans: They show the most positive factor mean values for the service counter $\left(\mu_{\mathrm{FM}}=0.91 ; \mu_{\mathrm{PM}}=1.11\right)$ and the most negative values for the self-service counter $\left(\mu_{\mathrm{FM}}=-0.80 ; \mu_{\mathrm{PM}}=0.61\right)$. They do their shopping preferably at the service counter $\left(\mu_{\mathrm{FM}}=2.25 ; \mu_{\mathrm{PM}}=1.73\right)$. The SC fans have the smallest share of $23.9 \%$ in the fresh meat sub-sample and also the smallest share in the processed meat subsample with $22.4 \%$. These shares are in line with data collected by LfL (2013) who state a share of $25 \%$. 

counter?

\section{Discussion of the cross tabulations and hypotheses}

The results of the cross tabulation analysis show that the self-service counter and the service counter are both the places where the target group for high welfare meat can be found doing their shopping. $54.0 \%$ of the fresh meat buyers buy both at the service counter and at the selfservice counter and $30.5 \%$ only buy fresh meat at the service counter. For the processed meat sub-sample, these proportions are $37.1 \%$ and $27.4 \%$. Finally, it can be seen that the target group for high welfare meat can be achieved better with a presentation on the service counter as more consumers of the target group can be found there due to the cluster of the Combiners. The results concerning the fresh meat sub-sample presented here are in line with Beck et al. (2007) and Schulze and Spiller (2007) and confirm the first hypothesis: High welfare meat should be sold at the service counter, while the sales potential at self-service counter appears less promising.

For processed meat, there are slightly different results as the self-service counter appears to be an option as point of sale as well. Thus, also the second hypothesis - that, according to LfL (2013), for processed meat, the service counter is not as important as it is for fresh meat - has also been verified through these results. The results of the cross tabulation for the processed meat sub-sample are not significant as well $(\mathrm{p}=0.144)$ and thus, no clear conclusion can be drawn. Selling the animal welfare products at the service counter and at the self-service counter might be an appropriate solution initially, as a clear preference could not be found for either one of the two counters. Regarding the processed meat buyers, $27.4 \%$ can be directly targeted at the service counter. High welfare processed meat can also be placed successfully at the self-service counter where $35.5 \%$ of the target group can be addressed. But according to Schulze and Spiller (2007) as well as Weyer (2005), consumers expect to find cheaper meat at the self-service counter. Therefore, placing high welfare processed meat products at the service counter is recommended as well.

An important requirement to sell high welfare processed and fresh meat is that the staff must be trained properly in order to meet the demand for an explanation of the product (Schulze and Spiller, 2007).

\section{Conclusions}

Although in the literature a demand for high welfare meat has been shown to exist (Lagerkvist and Hess, 2011), there remains the challenge of improving the availability of these products. In order to optimize the distribution of high welfare meat, it is important to find out at which kind of counter such products are best placed. In many countries like Germany, the USA and 
Canada, retailers have both a self-service counter and a service counter for fresh and processed meat. Due to space limitations, it is often not possible for retailers to place conventionally produced meat and high welfare meat at both. Thus, supermarkets with both kinds of counters have to decide at which counter they should best place high welfare meat. This paper aims to find out where high welfare meat should best be placed in Germany.

As the results of the present study show, at the self-service counter, high welfare meat might not be sold very successfully. This is supported by the fact that currently, in Germany products carrying the animal welfare label "Für mehr Tierschutz" are not sold well on a large scale at the self-service counter. The label was introduced in some stores in January 2013 and sold at the self-service counter ending in disappointing results. In 2014, most retailers stopped the introduction of high welfare meat and delisted labelled products.

There might be several reasons for this. Firstly, customers who buy their meat at the selfservice counter do not attach much importance to the meat they purchase. Secondly, customers expect low prices at the self-service counter (Schulze and Spiller, 2007; Weyer, 2005). Finally, lower quality is also expected (Schulze and Spiller, 2007). Consequently, to achieve long-term success and to establish the German animal welfare label we recommend that high welfare meat should be placed at the service counter as the results of the present research show that this is where the target group can be addressed best. So far, the service counter has not been tested for high welfare meat on a large scale.

Transferring these results to a broader context reveals that the introduction of niche products in retail in general is a challenge. As conventional products will not be substituted by the retailer, space restrictions in the supermarkets become problematic. The introduction of, e.g. products free of lactose or gluten and organic products, means doubling the shelf space which, of course, is not possible for each product. Products must be placed to maximize profit, or else the product will be delisted. Thus, market research is necessary in order to reduce the risks for retailers such as, for instance, a low turnover rate. Therefore, the introduction of those niche products needs to be prepared carefully concerning the whole marketing. Store tests would be an appropriate tool.

Apart from the question at which counter the products should be placed it is also necessary to communicate the advantages of the niche products, either by food package labelling at the self-service counter or by the staff at the service counter. Furthermore, advertising is crucial to boost the awareness of newly introduced products. The slow diffusion process of many sustainability labels is also due to low marketing budgets. Last but not least it is important to 

counter?

evaluate the willingness to pay in order to know at which price level the product can be sold successfully. Therefore, the results of this paper are an example for important marketing failures in the case of third party labels. Further research should analyze why marketing for label products can often be observed as unprofessional (e.g. free rider behaviors, small enterprises etc.).

\section{Acknowledgements}

The publication was funded as part of the project „Animal welfare label - Setup of an animal welfare programme in the pig meat chain adjusted to market conditions" by the Federal Ministry for Nutrition and Agriculture and the Federal Institute for Agriculture and Nutrition (BLE).

\section{References}

ALMA (Alberta Livestock and Meat Agency Ltd.) (2012). Canadian Consumer Retail Meat Study. Available at: http://alma.alberta.ca/cs/groups/alma/documents/document/ (accessed 23 July 2014)

Amine, A., Cadenat, S. (2003). Efficient retailer assortment: a consumer choice evaluation perspective. International Journal of Retail \& Distribution Management 31 (10), 486-497

Balling, R. (1990). Marketing-Konzeption für einen Markenartikel Rindfleisch. Agrarwirtschaft, Special Issue 125

Beck, A., Dylla R., Euen S., Hamm, U., Lücke, F.-K., Marx, B., Wild, S. (2007). Pökelstoffe in Öko-Fleischwaren, available at: http://orgprints.org/10466/ (accessed 23 October 2013)

Becker, T., Benner, E., Glitsch, K. (2000). Consumer perception of fresh meat quality in Germany. British Food Journal 102 (3), 246-266

Carlotti Jr S., Coe M.E., Perrey J. (2006). Designing and managing winning brand portfolios: profiting from proliferation. McKinsey Quarterly

Deimel, I., Franz, A., Frentrup, M., von Meyer, M., Spiller, A., Theuvsen, L. (2010). Perspektiven für ein Europäisches Tierschutzlabel, available at: download.ble.de/08HS010.pdf. (accessed 30 November 2012)

de Jonge J., van Trijp, H.C.M. (2013). Meeting Heterogeneity in Consumer Demand for Animal Welfare: A Reflection on Existing Knowledge and Implications for the Meat Sector. Journal of agricultural and environmental ethics 26 (3), 629-661

Everitt B.S. (1998). The Cambridge Dictionary of Statistics. Cambridge, Cambridge University Press

FAO (2009). The state of food and agriculture. Livestock in the balance. Rome, available at: http://www.fao.org/docrep/012/i0680e/i0680e.pdf (accessed 23 October 2013) 
Federal Statistical Office (2011). Statistical Yearbook 2011, available at: https://www.destatis.de/DE/Publikationen/StatistischesJahrbuch/StatistischesJahrbuch2011 .pdf?_blob=publicationFile (accessed 24 July 2014)

FTC (Federal Trade Commision) (2003). Slotting Allowances in the Retail Grocery Industry: Selected Case Studies in Five Product Categories. FTC Stuff Study, available at: http://www.ftc.gov/sites/default/files/documents/reports/use-slotting-allowances-retailgrocery-industry/slottingallowancerpt031114.pdf (accessed 20 July 2014)

Glitsch, K. (2000). Consumer perceptions of fresh meat quality: cross-national comparison. British Food Journal 102 (3), 177-194

Godfray, H.C.J., Beddington, J.R., Crute, I.R., Haddad, L., Lawrence, D., Muir, J.F. (2010). Food security: The challenge of feeding 9 billion people. Science 327 (5967), 812-818

Grunert, K.G. (2006). Future Trends and Consumer Lifestyles with respect to Meat Consumption, available at: http://www.icomst.helsinki.fi/previous_congress/ICoMST\%2052/ICoMST presentations/Thursday/Grunert.pdf (accessed 14 April 2014).

Hübner, A. H., Kuhn, H. (2012). Retail category management: State-of-the-art review of quantitative research and software applications in assortment and shelf space management. Omega 40 (2), 199-209

Kaiser, H.F. (1974). An index of factorial simplicity. Psychometrika, 39 (1), 31-36

Lagerkvist, C.J., Hess, S. (2011). A meta-analysis of consumer willingness to pay for farm animal welfare. European Review of Agricultural Economics, 38 (1), 55-78

LfL (Bayrische Landesanstalt für Landwirtschaft) (2013). Folienvorlage zum Kapitel 8 "Vieh und Fleisch" der Agrarmärkte 2013. Available at:

http://www.lfl.bayern.de/mam/cms07/iem/dateien/foliensatz_vieh_und_fleisch.pdf (accessed 14 February 2013)

LfL (Bayrische Landesanstalt für Landwirtschaft) / LEL (Schwäbisch Gmünd) (2013). Agrarmärkte 2013. Available at: http://www.lfl.bayern.de/mam/cms07/ publikationen/daten/schriftenreihe/agrarmaerkte2013.pdf (accessed 13 February 2014)

Lippke, S., Sniehotta, F.F. (2003). Ernährungsverhalten aus handlungsorientierter Sicht: Das Multistadienmodell der Gesundheitsverhaltensänderung. Zeitschrift für Gesundheitspsychologie, 11 (4), 143-152

Maruyama, M., Wu, L. (2014). Quantifying barriers impeding the diffusion of supermarkets in China: The role of shopping habits. Journal of Retailing and Consumer Services, 21 (3), 383-393

McEachern, M.G. and Schröder, M.J.A. (2002), "The role of livestock production ethics in consumer values towards meat", Journal of Agricultural and Environmental Ethics, Vol. 15 No. 2, pp. 221-237

Ngapo, T.M., Dransfield, E., Martin, J.-F., Magnusson, M., Bredahl, L., Nute, G.R. (2003). Consumer perceptions: pork and pig production. Insights from France, England, Sweden and Denmark. Meat Science, 66 (1), 125-134.

Padel, S., Foster, C. (2005). Exploring the Gap between Attitudes and Behaviour: Understanding why consumers buy or do not buy organic food", British Food Journal, Vol. 107 No 8, pp. 606-625 
Plaßmann, S., Hamm, U. (2009). Kaufbarriere Preis? - Analyse von Zahlungsbereitschaft und Kaufverhalten bei Öko-Lebensmitteln. Final report 06OE119. Universität Kassel, Germany, available at: http://orgprints.org/15745/1/15745-06OE119-uni_kassel-hamm-2009kaufbarriere_preis.pdf (accessed November 16, 2013)

Reardon, T., Timer, C.P., Minten, M. (2010). Supermarket revolution in Asia and emerging development strategies to include small farmers. Proceedings of the National Academy of Science of the United States of America, 109 (31), 12332-12337

Rodriguez, E., Berges, M., Casellas, K., Di Paola, R., Lupin, B., Garrido, L., Gentile, N. (2002). Consumer behavior and supermarkets in Argentina. Development Policy Review, $20(4), 429-439$

Roerink, A.-M. (2013). The Power of Meat 2013, available at: http://www.meatami. com/ht/a/GetDocumentAction/i/85035 (accessed 13 April 2014)

Röhr, A., Lüddecke, K., Drusch, S., Müller, M.J., von Alvensleben, R. (2005). Food quality and safety - consumer perception and public health concern. Food Control, 16 (8), 649-655

Schulze, B., Spiller, A. (2007). Wer geht noch an die Theke? Ergebnisse einer Verbraucherbefragung zu SB-Fleisch. Paper prepared for presentation at the 47th annual conference of the GEWISOLA (German Association of Agricultural Economists) and the 17th annual conference of the ÖGA (Austrian Association of Agricultural Economists), 'Changing Agricultural and Food Sector', Freising/Weihenstephan, Germany, September 26-28, 2007

Schulze, B., Spiller, A., Lemke, D. (2008a). Glücksschwein oder arme Sau? Die Einstellung der Verbaucher zur modernen Nutztierhaltung. In Spiller, A., Schulze, B. (Eds.). Zukunftsperspektiven der Fleischwirtschaft - Verbraucher, Märkte Geschäftsbeziehungen, pp. 465489. Göttingen, Universitätsverlag Göttingen

Schulze, H., Albersmeier, F., Gawron, J.-C., Spiller, A., Theuvsen, L. (2008b), Heterogeneity in the Evaluation of Quality Assurance Systems: The International Food Standard (IFS) in European Agribusiness. International Food and Agribusiness Management Review, 11 (3), 99-139

Smith, P.W. , Burns, D.J. (1997). Use of the service meat counter: an exploration. British Food Journal, 99 (7), 244-248

Spiller, A., Schulze, B. (2008). Trends im Verbraucherverhalten: Ein Forschungsüberblick zum Fleischkonsum. In: Spiller, A. and Schulze, B. (2008), Zukunftsperspektiven der Fleischwirtschaft - Verbraucher, Märkte Geschäftsbeziehungen. Göttingen, Universitätsverlag: Göttingen

Verbeke, W., Vackier, I. (2004). Profile and effects of consumer involvement in fresh meat. Meat Science, 67 (1), 159-168

Verbeke, W., Viaene, J. (1999). Ethical Challenges for Livestock Production: Meeting Consumers Concerns about Meat Safety and Animal Welfare. Journal of Agricultural and Environmental Ethics, 21 (2), 141-151

Weindlmaier, H. (1980). Verbrauchereinstellungen zu Fleisch und Fleischwaren sowie zu den Formen ihres Angebots. Ergebnisse einer Verbraucherbefragung in Bonn. Working Report No. 3 of Institut of Agricultural Business Operations, Bonn.

Weyer, F. (2005), Verbraucherverhalten und -einstellungen gegenüber Theken- und Selbstbedienungsware unter besonderer Berücksichtigung des Involvements und der Informationsnutzung. Eine theoriegeleitete, empirische Analyse am der Lebensmittel Wurst und Käse, Europäische Hochschulschriftenreihe 05, Vol. 3120, Frankfurt am Main a.o. 


\section{Appendix}

\section{Appendix A: Animal welfare clusters for the fresh meat sub-sample}

\begin{tabular}{|c|c|c|c|}
\hline & $\begin{array}{c}\text { Cluster } \mathbf{A}_{\mathrm{FM}} \text { : } \\
\text { AW indiffer- } \\
\text { ent }\end{array}$ & $\begin{array}{l}\text { Cluster } B_{\mathrm{FM}} \text { : } \\
\text { AW pragma- } \\
\text { tists }\end{array}$ & $\begin{array}{c}\text { Cluster } C_{F M} \\
A W \text { enthusi- } \\
\text { asts } \\
\end{array}$ \\
\hline $\begin{array}{l}\text { Size of the cluster, absolute and } \\
\text { in }(\%)\end{array}$ & $\begin{array}{c}133 \\
(42.6)\end{array}$ & $\begin{array}{c}88 \\
(28.2)\end{array}$ & $\begin{array}{c}91 \\
(29.2)\end{array}$ \\
\hline Factor $1_{\mathrm{FM}}$ : Attitude towards animal welfare ${ }^{* * *}$ & $\begin{array}{l}-0.84^{\text {bc }} \\
(0.647)\end{array}$ & $\begin{array}{l}0.39^{\mathrm{ac}} \\
(0.580)\end{array}$ & $\begin{array}{l}0.88^{\mathrm{ab}} \\
(0.564)\end{array}$ \\
\hline $\begin{array}{l}\text { I would like to have more information about livestock farming when } \\
\text { purchasing meat. }{ }^{1 * * *}\end{array}$ & $\begin{array}{l}-0.10^{\mathrm{bc}} \\
(1.313)\end{array}$ & $\begin{array}{l}1.48^{\mathrm{ac}} \\
(1.104)\end{array}$ & $\begin{array}{l}2.21^{\mathrm{ab}} \\
(0.888)\end{array}$ \\
\hline I strongly disapprove of livestock farming in big factory farms. ${ }^{1 * * *}$ & $\begin{array}{l}0.26^{\text {bc }} \\
(1.199)\end{array}$ & $\begin{array}{l}1.84^{\text {ac }} \\
(1.092)\end{array}$ & $\begin{array}{l}2.33^{\text {ab }} \\
(0.989)\end{array}$ \\
\hline $\begin{array}{l}\text { If I knew which meat originates from happy animals I would only buy } \\
\text { this meat. }{ }^{1 * * *}\end{array}$ & $\begin{array}{l}-0.16^{\mathrm{bc}} \\
(1.211)\end{array}$ & $\begin{array}{l}1.70^{\mathrm{ac}} \\
(1.176)\end{array}$ & $\begin{array}{l}2.13^{\mathrm{ab}} \\
(1.013)\end{array}$ \\
\hline $\begin{array}{l}\text { It makes me angry when thinking about how animals are kept in agricul- } \\
\text { ture today. }{ }^{1 * * *}\end{array}$ & $\begin{array}{l}-0.14^{\mathrm{bc}} \\
(1.393)\end{array}$ & $\begin{array}{c}1.26^{\mathrm{ac}} \\
(1.360)\end{array}$ & $\begin{array}{c}2.25^{\mathrm{ab}} \\
(1.131)\end{array}$ \\
\hline For me, animal welfare is a selection criterion when buying meat. ${ }^{1} * * *$ & $\begin{array}{l}-0.35^{\mathrm{bc}} \\
(1.231)\end{array}$ & $\begin{array}{l}1.35^{\mathrm{a}} \\
(1.194)\end{array}$ & $\begin{array}{c}1.58^{\mathrm{a}} \\
(1.375)\end{array}$ \\
\hline I have a problem with meat that originates from factory farms. ${ }^{1,4 * * *}$ & $\begin{array}{l}0.26^{\mathrm{bc}} \\
(1.235)\end{array}$ & $\begin{array}{l}1.16^{\mathrm{ac}} \\
(1.625)\end{array}$ & $\begin{array}{l}2.02^{\mathrm{ab}} \\
(1.238)\end{array}$ \\
\hline $\begin{array}{l}\text { I would change my buying behavior if there was meat on offer that was } \\
\text { labeled with an animal welfare label as well as the usual meat on offer in } \\
\text { my supermarket. }{ }^{1} * *\end{array}$ & $\begin{array}{l}-0.34^{\text {bc }} \\
(1.353)\end{array}$ & $\begin{array}{l}1.23^{\mathrm{ac}} \\
(1.311)\end{array}$ & $\begin{array}{l}1.93^{\mathrm{ab}} \\
(1.083)\end{array}$ \\
\hline $\begin{array}{l}\text { I find it disgusting how many animals are kept indoors in modern agri- } \\
\text { culture. }{ }^{1 * * *}\end{array}$ & $\begin{array}{c}0.36^{\mathrm{bc}} \\
(1.305)\end{array}$ & $\begin{array}{c}1.28^{\mathrm{ac}} \\
(1.389)\end{array}$ & $\begin{array}{l}2.08^{\mathrm{ab}} \\
(1.376)\end{array}$ \\
\hline Factory farming is bad for animal welfare. ${ }^{1} * * *$ & $\begin{array}{l}0.90^{\text {bc }} \\
(1.331)\end{array}$ & $\begin{array}{l}1.90^{\mathrm{ac}} \\
(1.305)\end{array}$ & $\begin{array}{l}2.43^{\mathrm{ab}} \\
(1.024)\end{array}$ \\
\hline When doing the shopping, I think about animal welfare. ${ }^{1,4 * * *}$ & $\begin{array}{l}-0.73^{\mathrm{bc}} \\
(1.219)\end{array}$ & $\begin{array}{c}0.28^{\mathrm{ac}} \\
(1.546)\end{array}$ & $\begin{array}{l}1.22^{\mathrm{ab}} \\
(1.569)\end{array}$ \\
\hline $\begin{array}{l}\text { I am interested in the living conditions of the animals that provide the } \\
\text { meat I purchase. }{ }^{1 * *}\end{array}$ & $\begin{array}{l}-0.41^{\mathrm{bc}} \\
(1.088)\end{array}$ & $\begin{array}{l}1.32^{\mathrm{a}} \\
(1.150)\end{array}$ & $\begin{array}{l}1.43^{\mathrm{a}} \\
(1.318)\end{array}$ \\
\hline To be honest, I spend a lot of time thinking about animal welfare. ${ }^{1,4 * * *}$ & $\begin{array}{l}-0.61^{\mathrm{bc}} \\
(1.341)\end{array}$ & $\begin{array}{c}0.56^{\mathrm{ac}} \\
(1.609)\end{array}$ & $\begin{array}{l}1.52^{\mathrm{ab}} \\
(1.368)\end{array}$ \\
\hline $\begin{array}{l}\text { I would like to buy more meat from livestock reared in appropriate } \\
\text { conditions, but I can seldom find any. } \text {. }_{* * *}\end{array}$ & $\begin{array}{l}-0.15^{\mathrm{bc}} \\
(1.145)\end{array}$ & $\begin{array}{c}1.11^{\mathrm{ac}} \\
(1.368)\end{array}$ & $\begin{array}{l}1.75^{\mathrm{ab}} \\
(1.305)\end{array}$ \\
\hline If possible, I buy meat from animals that are treated properly. ${ }^{1 * * *}$ & $\begin{array}{l}0.13^{\mathrm{bc}} \\
(1.131)\end{array}$ & $\begin{array}{c}1.80 \mathrm{a} \\
(1.052)\end{array}$ & $\begin{array}{c}1.78 \mathrm{a} \\
(0.998)\end{array}$ \\
\hline $\begin{array}{l}\text { In order to buy "high welfare meat" I would also go to the service coun- } \\
\text { ter. }{ }^{1 * * *}\end{array}$ & $\begin{array}{l}-0.05 \\
1.389 \\
\end{array}$ & $\begin{array}{c}1.78 \\
1.254\end{array}$ & $\begin{array}{c}2.20 \\
0.980\end{array}$ \\
\hline
\end{tabular}


counter?

\section{Appendix A continued}

\begin{tabular}{|c|c|c|c|}
\hline Factor $2_{\mathrm{FM}}$ : Perceived farm animal welfare situation $* * *$ & $\begin{array}{l}0.13^{\text {bc }} \\
(0.695)\end{array}$ & $\begin{array}{l}0.74^{\text {ac }} \\
(0.646)\end{array}$ & $\begin{array}{l}-0.98^{\mathrm{ab}} \\
(0.834)\end{array}$ \\
\hline In agriculture, animal welfare has greatly improved in recent years. ${ }^{1} * * *$ & $\begin{array}{l}0.53^{\mathrm{bc}} \\
(0.974)\end{array}$ & $\begin{array}{l}1.36^{\mathrm{ac}} \\
(0.925)\end{array}$ & $\begin{array}{l}-0.53^{\mathrm{ab}} \\
(1.294)\end{array}$ \\
\hline $\begin{array}{l}\text { In this country, sufficient attention is paid to animal welfare in livestock } \\
\text { farming. }{ }^{1 * * *}\end{array}$ & $\begin{array}{l}0.04^{\text {bc }} \\
(0.965)\end{array}$ & $\begin{array}{c}0.72^{\mathrm{ac}} \\
(1.039)\end{array}$ & $\begin{array}{l}-1.65^{\mathrm{ab}} \\
(1.079)\end{array}$ \\
\hline Factor $3_{\mathrm{FM}}$ : Knowledge and influence on livestock farming $* * *$ & $\begin{array}{l}-0.73^{\text {bc }} \\
(0.733)\end{array}$ & $\begin{array}{l}0.85^{\mathrm{ac}} \\
(\mathbf{0 . 6 3 3 )}\end{array}$ & $\begin{array}{l}0.29^{\text {ab }} \\
(0.750)\end{array}$ \\
\hline $\begin{array}{l}\text { Through my buying behavior, I have an influence on the mode of pro- } \\
\text { duction in agriculture. }{ }^{1} * * *\end{array}$ & $\begin{array}{l}-0.56^{\mathrm{ab}} \\
(1.189)\end{array}$ & $\begin{array}{c}1.91^{\text {ac }} \\
(0.967)\end{array}$ & $\begin{array}{c}1.07^{\mathrm{ab}} \\
(1.459)\end{array}$ \\
\hline $\begin{array}{l}\text { I am knowledgeable about the conditions in which animals are kept in } \\
\text { German agriculture. }{ }^{1} * *\end{array}$ & $\begin{array}{l}-0.23^{\mathrm{ab}} \\
(1.230)\end{array}$ & $\begin{array}{l}1.35^{\mathrm{ac}} \\
(1.051)\end{array}$ & $\begin{array}{c}0.76^{\mathrm{ab}} \\
(1.119)\end{array}$ \\
\hline
\end{tabular}

Significance level: $* * *=p \leq 0.001, * *=p \leq 0.01, *=p \leq 0.05$; factor mean values (standard deviation ); mean value; (standard deviation); letters mark a significant difference between groups, e. g., "Factory farming is bad for animal welfare": group A is significantly different to group B ("b") and group C ("c") (Tamhane's post hoc test T2 at a significance level 0.05)

${ }^{1}$ Scale from -3 "Not correct at all" to +3 "Fully correct"

${ }^{4}$ Statement was recoded

Source: authors' calculation 
Appenix B: Animal welfare clusters for the processed meat sub-sample

\begin{tabular}{|c|c|c|c|}
\hline & $\begin{array}{c}\text { Cluster } A_{P M}: \\
\text { AW indiffer- } \\
\text { ent }\end{array}$ & $\begin{array}{c}\text { Cluster } B_{\mathrm{PM}} \text { : } \\
\text { AW pragma- } \\
\text { tists }\end{array}$ & $\begin{array}{c}\text { Cluster } C_{P M} \text { : } \\
\text { AW enthusi- } \\
\text { asts }\end{array}$ \\
\hline $\begin{array}{l}\text { Size of cluster, absolute and } \\
\text { in }(\%)\end{array}$ & $\begin{array}{c}148 \\
(53.1)\end{array}$ & $\begin{array}{c}64 \\
(22.9)\end{array}$ & $\begin{array}{c}67 \\
(24.0)\end{array}$ \\
\hline Factor $1_{\mathrm{PM}}$ : Attitude towards animal welfare $* * *$ & $\begin{array}{l}-0.69^{\text {bc }} \\
(0.682)\end{array}$ & $\begin{array}{l}0.42^{\mathrm{ac}} \\
(0.618)\end{array}$ & $\begin{array}{l}1.07^{\mathrm{ab}} \\
(0.487)\end{array}$ \\
\hline $\begin{array}{l}\text { It makes me angry when thinking about how animals are kept in agricul- } \\
\text { ture today. }{ }^{* * *}\end{array}$ & $\begin{array}{l}-0.05^{\mathrm{bc}} \\
(1.271)\end{array}$ & $\begin{array}{c}0.92^{\mathrm{ac}} \\
(1.349)\end{array}$ & $\begin{array}{c}2.13^{\mathrm{ab}} \\
(1.072)\end{array}$ \\
\hline $\begin{array}{l}\text { I find it sad that nowadays so many animals are kept in the narrowest of } \\
\text { spaces so that we can buy cheap meat. }{ }^{1 * * *}\end{array}$ & $\begin{array}{l}0.56^{\mathrm{bc}} \\
(1.252)\end{array}$ & $\begin{array}{l}1.95^{\text {ac }} \\
(0.999)\end{array}$ & $\begin{array}{l}2.69^{\mathrm{ab}} \\
(0.608)\end{array}$ \\
\hline Factory farming is bad for animal welfare. ${ }^{1 * * *}$ & $\begin{array}{l}0.68^{\mathrm{bc}} \\
(1.247)\end{array}$ & $\begin{array}{l}1.92^{\mathrm{ac}} \\
(1.212)\end{array}$ & $\begin{array}{c}2.58^{\mathrm{ab}} \\
(0.924)\end{array}$ \\
\hline I strongly disapprove of livestock farming in big factory farms. ${ }^{1} * * *$ & $\begin{array}{l}0.18^{\mathrm{bc}} \\
(1.340)\end{array}$ & $\begin{array}{l}1.70^{\mathrm{a}} \\
(1.341)\end{array}$ & $\begin{array}{c}2.15^{\mathrm{a}} \\
(1.317)\end{array}$ \\
\hline $\begin{array}{l}\text { I find it disgusting how many animals are kept indoors in modern agri- } \\
\text { culture. }{ }^{1 * * *}\end{array}$ & $\begin{array}{c}0.22^{\mathrm{bc}} \\
(1.368)\end{array}$ & $\begin{array}{l}1.25^{\mathrm{ac}} \\
(1.553)\end{array}$ & $\begin{array}{c}2.06^{\mathrm{ab}} \\
(1.099)\end{array}$ \\
\hline $\begin{array}{l}\text { For me, animal welfare is a selection criterion when buying processed } \\
\text { meat. }{ }^{* * *}\end{array}$ & $\begin{array}{l}-0.51^{\mathrm{bc}} \\
(1.259)\end{array}$ & $\begin{array}{c}0.84^{\mathrm{ac}} \\
(1.158)\end{array}$ & $\begin{array}{l}1.67^{\mathrm{ab}} \\
(1.211)\end{array}$ \\
\hline $\begin{array}{l}\text { If I knew which processed meat products originates from happy animals } \\
\text { I would only buy this processed meat products. }{ }^{1 * * *}\end{array}$ & $\begin{array}{l}-0.22^{\mathrm{bc}} \\
(1.232)\end{array}$ & $\begin{array}{l}1.38^{\mathrm{ac}} \\
(1.303)\end{array}$ & $\begin{array}{l}2.21^{\mathrm{ab}} \\
(0.946)\end{array}$ \\
\hline $\begin{array}{l}\text { In order to buy "high welfare processed meat" I would also go to the } \\
\text { service counter. }{ }^{1 * * *}\end{array}$ & $\begin{array}{l}0.12^{\mathrm{c}} \\
1.730\end{array}$ & $\begin{array}{c}0.57 \\
1.561\end{array}$ & $\begin{array}{l}1.21^{\mathrm{a}} \\
1.760\end{array}$ \\
\hline I have a problem with meat that originates from factory farms. ${ }^{1,4 * * *}$ & $\begin{array}{c}0.21^{\mathrm{bc}} \\
(1.090)\end{array}$ & $\begin{array}{c}1.22^{\mathrm{ac}} \\
(1.588)\end{array}$ & $\begin{array}{c}2.24^{\mathrm{ab}} \\
(1.156)\end{array}$ \\
\hline $\begin{array}{l}\text { I would like to have more information about livestock farming when } \\
\text { purchasing processed meat product. }^{1} * * *\end{array}$ & $\begin{array}{l}-0.24^{\mathrm{bc}} \\
(1.296)\end{array}$ & $\begin{array}{l}1.59^{\mathrm{a}} \\
(1.205)\end{array}$ & $\begin{array}{c}2.00^{\mathrm{a}} \\
(1.000)\end{array}$ \\
\hline $\begin{array}{l}\text { I would change my buying behavior if there was processed meat on offer } \\
\text { that was labeled with an animal welfare label as well as the usual meat } \\
\text { on offer in my supermarket. }{ }^{1 * * *}\end{array}$ & $\begin{array}{l}-0.32^{\mathrm{bc}} \\
(1.289)\end{array}$ & $\begin{array}{l}1.06^{\mathrm{ac}} \\
(1.296)\end{array}$ & $\begin{array}{l}1.82^{\mathrm{ab}} \\
(1.127)\end{array}$ \\
\hline To be honest, I spend a lot of time thinking about animal welfare. ${ }^{1,4 * * *}$ & $\begin{array}{l}-0.60^{\mathrm{bc}} \\
(1.329)\end{array}$ & $\begin{array}{c}0.58^{\mathrm{ac}} \\
(1.499)\end{array}$ & $\begin{array}{c}1.81^{\mathrm{ab}} \\
(1.282)\end{array}$ \\
\hline Factor $2_{\mathrm{PM}}$ : Perceived farm animal welfare situation (conf.) ${ }^{* * *}$ & $\begin{array}{l}0.04^{\text {bc }} \\
(0.772)\end{array}$ & $\begin{array}{l}0.91^{\text {ac }} \\
(0.631)\end{array}$ & $\begin{array}{l}-0.88^{a b} \\
(0.824)\end{array}$ \\
\hline In agriculture, animal welfare has greatly improved in recent years. ${ }^{1} * * *$ & $\begin{array}{l}0.43^{\mathrm{bc}} \\
(0.919)\end{array}$ & $\begin{array}{l}1.44^{\mathrm{ac}} \\
(0.852)\end{array}$ & $\begin{array}{l}-0.25^{\mathrm{ab}} \\
(1.146)\end{array}$ \\
\hline $\begin{array}{l}\text { In this country, sufficient attention is paid to animal welfare in livestock } \\
\text { farming. }{ }^{1 * * *}\end{array}$ & $\begin{array}{l}-0.05^{\text {bc }} \\
(0.999)\end{array}$ & $\begin{array}{c}0.73^{\mathrm{ac}} \\
(1.043)\end{array}$ & $\begin{array}{l}-1.31^{\mathrm{ab}} \\
(1.003)\end{array}$ \\
\hline $\begin{array}{l}\text { Factor } 3_{\mathrm{PM}}: \text { Knowledge and influence on livestock farming } \\
\text { (conf.) } *_{* *}\end{array}$ & $\begin{array}{l}-0.55^{\text {bc }} \\
(0.773)\end{array}$ & $\begin{array}{l}0.80^{\mathrm{a}} \\
(0.698)\end{array}$ & $\begin{array}{c}0.48^{\mathrm{a}} \\
(0.777)\end{array}$ \\
\hline $\begin{array}{l}\text { Through my buying behavior, I have an influence on the mode of pro- } \\
\text { duction in agriculture. }{ }^{1 * * *}\end{array}$ & $\begin{array}{l}-0.41^{\mathrm{bc}} \\
(1.256)\end{array}$ & $\begin{array}{c}1.45 \mathrm{a} \\
(1.246) \\
\end{array}$ & $\begin{array}{c}1.15 \mathrm{a} \\
(1.209)\end{array}$ \\
\hline $\begin{array}{l}\text { I am knowledgeable about the conditions in which animals are kept in } \\
\text { German agriculture. }{ }^{1} * *\end{array}$ & $\begin{array}{l}-0.12^{\mathrm{bc}} \\
(1.256)\end{array}$ & $\begin{array}{l}1.38^{\mathrm{ac}} \\
(0.900)\end{array}$ & $\begin{array}{l}0.88^{\mathrm{ab}} \\
(1.200)\end{array}$ \\
\hline
\end{tabular}

Significance level: $* * *=p \leq 0.001, * *=p \leq 0.01, *=p \leq 0.05$; factor mean values (standard deviation ); mean value; (standard deviation); letters mark a significant difference between groups, e. g., "Factory farming is bad for animal welfare": group A is significant different to group B ("b") and group C ("c") (Tamhane's post hoc test T2 at a significance level 0.05)

${ }^{1}$ Scale from -3 "Not correct at all" to +3 "Fully correct"

${ }^{4}$ Statement was recoded

Source: authors' calculation 


\section{Abschlussbetrachtung}

Das Verbraucherinteresse an Qualität und bestimmten Produkteigenschaften steigt, ebenso die Verunsicherung der Konsumenten (BVE 2011, Caswell und Joseph 2008, Grunert 2005, Zühlsdorf et al. 2013). Gründe dafür sind in den einleitend genannten Lebensmittelskandalen, aber auch in der Globalisierung und politischen Maßnahmen (TTIP) zu finden. Für viele Verbraucher ist es daher wichtig, Produktinformationen über die Lebensmittelkennzeichnung zu erhalten (Fernqvist und Ekelund 2014). Bei der Kennzeichnung von Produkten spielen Vertrauenseigenschaften wie Produkt- oder Prozesseigenschaften eine wichtige Rolle und können ein wesentliches Einkaufskriterium sein (ebd.). Daher haben einige Elemente der Lebensmittelkennzeichnung das Ziel, Vertrauenseigenschaften in Sucheigenschaften umzuwandeln (Caswell and Mojduszka 1996, Caswell und Padberg 1992, Jahn et al. 2005), um so Informationsasymmetrien abzubauen (Antle 2001, Darby und Karni 1973, Nelson 1970) und Produktbesonderheiten hervorzuheben. Für einen funktionierenden Markt ist es notwendig, dass unterschiedliche Qualitäten deutlich gemacht werden. Nur so kann gewährleistet werden, dass lautere Wettbewerber nicht aus dem Markt verdrängt werden (Akerlof 1970) und dass unterschiedliche Qualitäten angeboten werden.

Vor diesem Hintergrund war es das Ziel der Dissertation, verschiedene Labelling Policies, die bisher nicht im Fokus der Konsumentenforschung standen, mittels empirischer Verbraucherbefragungen zu untersuchen, zu bewerten und daraus Empfehlungen für Entscheidungsträger in der Lebensmittelkennzeichnung abzuleiten. Die drei zu untersuchenden Forschungsfragen lauteten daher:

- Wie können Prozess- und Produkteigenschaften differenziert auf Lebensmittelverpackungen kommuniziert werden? (Teil I: Potentials for multi-level food labelling systems)

- Wie werden verschiedene Kennzeichnungselemente auf Produktverpackungen, die Schlüsselinformationen für Verbraucher darstellen, von Verbrauchern wahrgenommen und beurteilt? (Teil II: Consumer understanding of food packaging)

- Welches sind für die Nischenprodukte Tierschutzfleisch und Weidemilch geeignete Marketingstrategien und durch welche Merkmale lassen sich Zielgruppen charakterisieren? (Teil III: Marketing strategies for niche products)

Kapitel 1 beschäftigte sich daher zunächst mit der Entwicklung einfacher und transparenter Kennzeichnungssysteme, die Verbrauchern wichtige Produktinformationen liefern. Ist eine 
Abweichung von Verbraucherwahrnehmung der Kennzeichnung und der tatsächlichen Beschaffenheit und Qualität eines Produktes zu erkennen, ist eine Änderung der Kennzeichnungsregulierung bzw. der Kennzeichnungspraxis von Nöten (Palma et al. 2014). Dieses zweite Erfordernis wurde in Kapitel 2 aufgegriffen. Zudem sind als dritter Faktor Marketingstrategien essenziell, um Produkte am Lebensmittelmarkt erfolgreich etablieren zu können. Kapitel 3 diente daher der Ermittlung und Charakterisierung der Zielgruppen, wodurch Marketingstrategien entwickelt bzw. angepasst werden können.

In den nächsten drei Abschnitten werden die Kernergebnisse der Studien in Zusammenhang mit den entsprechenden Schlussfolgerungen dargestellt. Zudem werden jeweils Implikationen für die Agrar- und Ernährungswirtschaft abgeleitet und Politikempfehlungen gegeben. Ergänzend werden Limitationen der Arbeit angeführt sowie ein Überblick über weiteren Forschungsbedarf gegeben. Die Dissertation schließt mit einem Fazit und Ausblick auf zukünftige Entwicklungen des Lebensmittelmarktes.

\section{Potentials for multi-level food labelling systems}

Die vorliegende Dissertation analysiert in Teil 1 die Potenziale von mehrstufigen Lebensmittelkennzeichnungssystemen und dient damit der Beantwortung der ersten Forschungsfrage. Derzeit dominieren binäre Labelsysteme wie z. B. das Bio-Siegel den Lebensmittelmarkt. Viele Prozesseigenschaften sind allerdings nicht durch binäre, sondern kontinuierliche Eigenschaften gekennzeichnet, wie bspw. Tierschutz oder Umweltverträglichkeit. Daher ist ein mehrstufiges Label aus wissenschaftlicher Sicht besser geeignet, solche Charakteristika zu kommunizieren. Ein mehrstufiges Labelsystem liefert für Verbraucher mehr Informationen, ist aber zugleich komplexer. Bisher wurde die Wirkung mehrstufiger Label auf Konsumenten nicht untersucht. Deshalb wurden im ersten Teil der Dissertation Potenziale für mehrstufige Labelsysteme in der Lebensmittelkennzeichnung herausgearbeitet.

Für die Etablierung eines mehrstufigen Labelsystems ist es zunächst wichtig zu untersuchen, ob ein solches System funktionieren kann. Die signifikant unterschiedlichen Zahlungsbereitschaften für ein zweistufiges Label, die im ersten Artikels (I.1: Multi-level labelling: too complex for consumers?) festgestellt wurden, lassen den Schluss zu, dass ein mehrstufiges System erfolgsversprechend ist, wenn ausführliche Informationen geliefert werden. Mit steigendem Standard der Haltungsbedingungen für ein Tierschutzlabel steigt auch die hypothetische Zahlungsbereitschaft der Konsumenten.

Es ist anzumerken, dass die Einführung eines mehrstufigen Labelsystems nur sinnvoll ist, wenn es gegenüber einem binären System Vorteile aufweist. Artikel I.2 (Developing food 
labellig strategies with the help of extremeness aversion) untersuchte daher Relationen von Marktanteilen gelabelter Tierschutzprodukte zwischen einem binären und einem mehrstufigen Labelsystem. Die Ergebnisse zeigen, dass die Einführung eines mehrstufigen Labelsystems, zu einem geringeren Anteil der Nicht-Käufer gelabelter Produkte im Vergleich zu einem binären Label geringer führt. Vor allem das Preiseinstiegssegment generiert einen relativ hohen Marktanteil. Die Ergebnisse lassen sich mit der Theorie zur Extremeness Aversion erklären: Eine Ausweitung des Produktangebots führt dazu, dass der Marktanteil für Produkte im mittleren Preissegment zunimmt (Simonson und Tversky 1992, Smith und Nagle 1995, Tversky und Simonson 1993). Beträgt der Nicht-Käufer-Anteil bei dem binären Labelsystem knapp $70 \%$, so liegt dieser Anteil bei einem mehrstufigen System bei etwa $24 \%$. Darüber hinaus zeigen eigene Marktanteilsberechnungen, dass der Tierschutz mit einem mehrstufigen Labelsystem stärker verbessert werden kann als mit einem binären Label. Zusätzlich kann der Gesamtumsatz bei dem mehrstufigen System deutlich gesteigert werden, wie die Berechnungen offen legen.

Es besteht weiterer Forschungsbedarf, der das Labeldesign betrifft. Die Darstellung der Stufigkeit, z. B. durch Sterne oder Balken, hat einen entscheidenden Einfluss auf das Verbraucherverständnis der Stufigkeit (Heinzle and Wüstenhagen 2010), weshalb vertiefende Studien erforderlich sind. Es ist zudem limitierend anzumerken, dass in der Studie ein zweistufiges Label analysiert wurde und daher Rückschlüsse für ein bspw. fünfstufiges Labelsystem nur bedingt zulässig sind. Auch hier besteht weiterer Forschungsbedarf der systematischen Analyse verschiedener Abstufungen.

$\mathrm{Zu}$ Bedenken ist bei der Einführung eines mehrstufigen Labellingsystems, dass für die verschiedenen Stufen differenzierte Zahlungsbereitschaften bei den Verbrauchern erzielt werden müssen. Für die hypothetisch ermittelten Zahlungsbereitschaften der Studien kann trotz angewandten Cheap Talk Skripts nicht ausgeschlossen werden, dass die Zahlungsbereitschaften auf Grund sozialer Erwünschtheit überschätzt wurden (Johansson-Stenman und Svedsäter 2012, Vlaev 2012). Nur wenn die Mehrkosten, die durch höhere Produktionsstandards entstehen, monetär ausgeglichen werden, können sich mehrere Stufen eines Labels etablieren. Zudem ist es notwendig zu ermitteln, an welchen Stellen der Supply Chain welche zusätzlichen Kosten durch die höheren Standards entstehen, um Marktpreise anpassen zu können. Auf Grund der fehlenden Datengrundlage können zum Deckungsbeitrag keine Angaben gemacht werden. Dies sollte in weiteren Studien berücksichtigt werden. 
Die Ergebnisse der ersten drei Artikel decken Potenziale für mehrstufige Labelsysteme auf, die für Entscheidungsträger in der Politik wichtige Erkenntnisse aufzeigen: Im Vergleich zu einstufigen Systemen können verschiedene Prozessstandards differenzierter kommuniziert werden, wodurch Marktversagen verhindert werden kann. Zudem können ethische Aspekte wie Tierschutz gesamtgesellschaftlich verbessert werden, indem höhere Marktanteile für gelabelte Produkte realisiert werden können. Für Produzenten, die höhere Produktionsstandards haben, ist eine mehrstufige Labelstrategie vorteilhaft, da sie bei höheren Standards auch einen finanziellen Mehrwert erzielen können. Dies schafft zusätzlich Anreize, Produktionsstandards anzuheben. Zudem ist die Eintrittsbarriere für Produzenten geringer, wenn für das Labelsegment mit den niedrigsten Anforderungen zunächst weniger Investitionen getätigt werden müssen.

\section{Consumer understanding of food packaging}

Im zweiten Teil der Arbeit standen ergänzend zu den Potenzialen von Labellingsystemen weitere Kennzeichnungselemente im Fokus, die Schlüsselinformationen für Verbraucher beim Einkauf darstellen können. Nur wenn Schlüsselinformationen Produktvorstellungen bei Verbrauchern hervorrufen, die der Produktbeschaffenheit entsprechen, sind diese geeignet, um das Produkt von anderen unterscheiden und abgrenzen zu können. Die Ergebnisse der vor diesem Hintergrund durchgeführten Studien beantworten die zweite Forschungsfrage.

Bisher ist die Wirkung einer Vielzahl von Kennzeichnungselementen wie z. B. Produktbezeichnungen oder Clean Label nicht empirisch untersucht worden. Die Ergebnisse des zweiten Kapitels deuten darauf hin, dass die derzeitig existierende Kennzeichnungspraxis Schwächen aufweist. Die ersten beiden Beiträge (II.1: Verbraucherverständnis von Verkehrsbezeichnungen und II.2: Verbraucherverständnis der Gestaltung von Lebensmittelverpackungen) legen offen, dass die analysierten Verkehrsbezeichnungen, Produkt- und Zutatenabbildungen in Zusammenhang mit dem Zutatenverzeichnis und Clean Labeln zu einer Fehleinschätzung der Produktbeschaffenheit bei Verbrauchern führen. Dies birgt die Gefahr, dass Verbraucher enttäuscht werden, wenn sie die Abweichung zwischen ihrer Auffassung und der tatsächlichen Produktbeschaffenheit wahrnehmen (Oakes und Slotterback 2001). Bei weitgehenden Fällen des Enttäuschungsempfindens können Verbraucher sich sogar getäuscht fühlen. Neben den Abweichungen zwischen Produktbeschaffenheit und Kennzeichnung ist es vermehrt zu beobachten, dass Verbrauchererwartungen oft über die zu Grunde liegenden Produkt- und Prozesseigenschaften hinausgehen (Canavari et al. 2009, Chandon und Wansink 2007, von Meyer-Höfer et al. 2013). Dies bestätigen die Ergebnisse in Artikel II.3 (Zum Ver- 
braucherverständnis von Alpen- und Weidemilch). Verbraucher haben an eine Weidemilch nicht nur die Erwartung, dass die Kühe auf der Weide stehen, sondern auch konkrete Anforderungen an die Fütterung. Dies ist ebenfalls in der Forschung bei der Kennzeichnung von Nährwerten (Chandon und Wansink 2007: erwartet wird, dass ein gesundes Lebensmittel zugleich wenig Kalorien enthält) und ökologischen Produkten (Canavari et al. 2009: erwartet wird, dass biologisch erzeugte Lebensmittel besser schmecken, obwohl dies wissenschaftlich nicht eindeutig belegt ist) bekannt und wird als „Halo-Effekt“ bezeichnet. Gefördert wird der Halo-Effekt durch ein entsprechendes Marketing wie z. B. eine romantisierende Verpackungsgestaltung. Ist bspw. im Hintergrund auf einer Schokoladenverpackung ein Gebirgszug zu erkennen, können Verbraucher diese Abbildung mit den Alpen und der Verwendung von Alpenmilch assoziieren. Daher sollten sich Hersteller und Verarbeiter auch mit „impliziter Konsumententäuschung“ (Gröppel-Klein 2012) beschäftigen, die bei Verbrauchern zu Enttäuschung führen kann.

Im Sinne des Ziels eines zufriedenen und loyalen Kundenstamms ist es für Hersteller empfehlenswert, sich bei der Auslobung von Produkt- oder Prozesseigenschaften auf Lebensmittelverpackungen mit den Verbrauchererwartungen auseinanderzusetzen. Bei Weide- und Alpenmilch sollten Branchenstandards festgelegt werden, damit keine Verdrängung lauterer Wettbewerber aus dem Markt stattfindet. Ist es jedoch Branchen wie dem Milchsektor nicht möglich, selbst Standards festzusetzen, die den fairen Wettbewerb regeln, sollte der Staat regulierend eingreifen und dies übernehmen. Ähnliches ist in der Bio-Branche in den 1980er Jahren zu beobachten gewesen. Auf Grund des zu erzielenden Mehrpreises für Bioprodukte wurden „Pseudo-Bioprodukte“ (Dabbert 2001) angeboten, was in Intransparenz für Verbraucher resultierte. Um einen funktionierenden Markt mit einem Mindestmaß an Transparenz zu gewährleisten, wurde 1991 die EU-Bio-Kennzeichnungsverordnung eingeführt (ebd.).

Es ist zu befürworten, dass von der Gesetzgebung, aber auch in der Rechtsprechung und auf Herstellerseite häufiger demoskopische Untersuchungen eingesetzt werden, um die Verbraucherauffassung von Schlüsselinformationen festzustellen und die Kennzeichnungspraxis bzw. Regulierungen anzupassen. Denkbar wäre auch die Entwicklung eines Standardtools, mit dem Verbraucherauffassungen von Kennzeichnungen systematisch und repräsentativ abgefragt werden. Das Tool wäre zunächst teuer in der ersten Entwicklung, für Folgebefragungen würden allerdings deutlich geringere Kosten anfallen. Das Argument einer hohen finanziellen Belastung ist nachvollziehbar, aber auf Grund der Verbraucherverunsicherung zu vernachlässigen. Zudem entstehen durch gerichtliche Wettbewerbsverfahren (§ 5 UWG) Kosten in nicht unerheblichem Ausmaß. 
Da die Arbeit der Deutschen Lebensmittelbuch-Kommission (DLMBK) während der Erstellung der Artikel II.1 und II.2 weniger im Fokus stand als aktuell, werden in diesem Abschnitt umstrittene Aspekte die DLMBK betreffend diskutiert. Die DLMBK legt in ihren Leitsätzen für viele Lebensmittel Herstellung, Beschaffenheit und Merkmale fest (§ 15 LFGB). Das Gremium besteht aus 32 Mitgliedern, die sich paritätisch aus dem Kreis von Wissenschaftlern, Verbrauchervertretern, Vertretern der Lebensmittelwirtschaft und der Lebensmittelüberwachung zusammensetzen. Stimmen alle Vertreter der Lebensmittelwirtschaft gegen einen Beschluss, kann dieser auf Grund der Geschäftsordnung der DLMBK verhindert werden $(\S 12$ der Geschäftsordnung der Deutschen Lebensmittelbuchkommission). Damit können materiell Private die Änderung von Leitsätzen verhindern, da Privatpersonen in dem Gremium nicht ausschließlich konsultativ wirken. Rechtswissenschaftler stellen daher die demokratische Legitimation der DLMBK in Frage, da die Leitsätze wie Gesetze wirken, ohne solche zu sein (Rixen 2014). Es bleibt jedoch an dieser Stelle offen, ob die Rechtsprechung zu dem Schluss kommt, dass es der DLMBK an Legitimation mangelt. Dies würde zu einer erheblichen Umstrukturierung im Lebensmittelrecht führen, da die Leitsätze in Produktverordnungen überführt werden müssten oder die DLMBK als ein staatlich legimitiertes Gremium institutionalisiert werden muss. Zweifellos ist die angestoßene Debatte dahingehend positiv zu bewerten, dass die derzeitig geltenden Vorschriften diskutiert werden. Zudem haben die Fachausschüsse der DLMBK, die für die Erstellung und Überarbeitung der Leitsätze für Lebensmittel zuständig sind, inzwischen begonnen, die Leitsätze zu überarbeiten (Rehlender 2012). Inzwischen wurde auch eine wissenschaftliche Evaluierung der Leitsätze des Deutschen Lebensmittelbuches und der DLMBK durch das Bundesministerium für Ernährung und Landwirtschaft veranlasst und durchgeführt. Nach öffentlicher Ausschreibung des Auftrags erhielt das Bonner Beratungsunternehmen AFC Public Services den Zuschlag. Ergebnisse sind bisher nicht veröffentlicht. $^{18}$

Einschränkend ist für Kapitel 2 anzuführen, dass in den Artikeln II.1 und II.2 Fallbeispiele analysiert werden, die nicht die Breite möglicher Probleme der Aufmachung und Bezeichnung von Produkten berücksichtigen. Die Artikel dienen vielmehr der grundsätzlichen Beleuchtung der Problematik. Zur systematischen Analyse von Kennzeichnungselementen sollte wie oben beschrieben ein Standardtool entwickelt werden, mit dem das Verbraucherverständ-

\footnotetext{
${ }^{18}$ Stand: 11.03.2015. http://www.topagrar.com/news/Home-top-News-Lebensmittelbuch-Kommission-Vorwurfder-Geheimhaltung-unhaltbar-1722793.html
} 
nis für eine Vielzahl problematischer Aufmachungen und Bezeichnungen analysiert werden kann.

Interessant wäre es, in weiteren Studien zu untersuchen, welche Elemente von Verbrauchern realiter im Supermarkt bei der Kaufentscheidung herangezogen werden und an welcher Stelle die Informationen platziert sein sollten, um die Aufmerksamkeit der Verbraucher zu erwirken. Derzeit ist bspw. nicht geregelt, an welcher Stelle die Verkehrsbezeichnung platziert sein muss.

Abschließend ist für Kapitel 2 anzumerken, dass die Lebensmittelkennzeichnung zwar die Interessen von Verbrauchern, Produzenten und Herstellern gleichermaßen schützen muss, im Zweifel wiegen jedoch die Verbraucherinteressen stärker, da diese vor Irreführung und Täuschung geschützt werden müssen (Hohmann 1994). Maßgeblich für die Verkehrsauffassung ist der „durchschnittlich informierte, situationsadäquat aufmerksame und verständige Verbraucher“ (z. B. GRUR Int. 1999, 354ff, „Sektkellerei Kessler“). Eine Rückkehr zum Leitbild des flüchtigen Verbrauchers (BGH GRUR 1959, 366 - Englisch Lavendel), das bis Mitte der 1990er Jahre Anwendung fand, ist auf Grund der Europäisierung nicht zu erwarten. Es kann letztlich nicht geklärt werden, inwieweit Verbraucher selbst verantwortlich sind, Informationen einzuholen und wie Hersteller Informationen bereitstellen sollten. Derzeit verwendete Schlüsselinformationen auf Lebensmittelverpackungen sollten jedoch auf Grund der Ergebnisse des zweiten Kapitels von rechtlicher, aber auch von Hersteller- bzw. Produzentenseite überdacht werden.

\section{Marketing strategies for niche products}

Während Teil 2 der Dissertation aufgezeigt hat, dass die derzeitige Lebensmittelkennzeichnungspraxis Defizite aufweist, stehen im dritten Teil der Arbeit exkursartig Marketingstrategien für die Nischenprodukte Weidemilch und Tierschutzfleisch vertiefend im Fokus. Dies ist besonders vor dem Hintergrund wichtig, dass Prozess- und Produkteigenschaften, die als Vertrauenseigenschaften charakterisiert sind, für Verbraucher immer wichtiger werden (Caswell und Joseph 2008, Fernqvist und Ekelund 2014). Neben der Herausforderung, unterschiedliche Prozess- und Produktqualitäten durch Lebensmittelkennzeichnung zu verdeutlichen (Akerlof 1970), ist es ebenfalls wichtig herauszufinden, wie entsprechende Produkte erfolgreich auf dem Markt eingeführt und etabliert werden können. Die Ergebnisse des dritten Teils der Dissertation beantworten die dritte Forschungsfrage.

Artikel III.1 lässt den Schluss zu, dass sich die Diskussion um Weidemilch verstärken wird. Die sich intensivierende Debatte um Stallhaltungssysteme bei Milchkühen liegt darin begrün- 
det, dass die Anzahl der Milchviehbetriebe abnimmt, aber die Herdengrößen zunehmen (Statistisches Bundesamt 2011). Bei einer zunehmenden Anzahl an Milchkühen ist es für Betriebe schwierig, genügend arrondierte Weideflächen zu finden, die eine Weidehaltung im Sommer ermöglichen. Die Ergebnisse von Art III.1 legen offen, dass Weidehaltung für viele Verbraucher sehr wichtig und positiv konnotiert ist. Es ist zu vermuten, dass vielen Konsumenten nicht bewusst ist, dass der Großteil der Milchkühe in Deutschland keine extensive Weidehaltung erfahren (Sprengel 2009). Es wird ein deutliches Marktpotenzial für die Vermarktung von Weidemilch aufgedeckt. 28,1\% der deutschen Konsumenten weisen eine signifikant höhere Zahlungsbereitschaft für Weidemilch auf. Doch nur eine transparente Kennzeichnung der zu Grunde liegenden Prozessstandards wird dazu beitragen, dass höhere Preise für Weidemilch erzielt werden (Kehlbacher 2012). Ein finanzieller Anreiz ist jedoch Voraussetzung, damit Landwirte weiterhin bereit sind, den aus der Weidehaltung resultierenden Mehraufwand zu betreiben.

Derzeit hat die Milchbranche ein gutes Image (Schleyer 2013). Es ist jedoch nicht ausgeschlossen, dass sich ähnliche Entwicklungen abzeichnen wie in der Fleischbranche, deren Image derzeit eher negativ geprägt ist derzeit ein eher negatives Image hat (ebd.). Daher ist es von Vorteil, wenn die Milchbranche auf die Bedürfnisse der Konsumenten reagiert und eine Weidemilch anbietet, deren Prozessstandards transparent sind. Die Haltungspraxis sollte auf Verpackungen realitätsnah kommuniziert und romantisierende Bilder (z. B. weidende Kühe) vermieden werden, wenn diese nicht dem Herstellungsprozess entsprechen. Ist es der Milchbranche in Deutschland nicht möglich, einheitliche Standards für Weidemilch festzulegen, sollte im Sinne eines fairen Wettbewerbes und funktionierenden Marktes der Staat regulierend eingreifen und das Produkt „Weidemilch“ gesetzlich definieren.

Das zweite betrachtete Nischenprodukt ist Tierschutzfleisch. Mehrere wissenschaftliche Untersuchungen belegen, dass es eine relativ große Zielgruppe für Tierschutzfleisch gibt (Harper und Makatouni 2002, Kehlbacher et al. 2012, Lagerkvist und Hess 2011, Schulze et al. 2008). Obwohl die Nachfrage mittels Konsumentenbefragungen in Deutschland auf etwa $20 \%$ geschätzt wird (Schulze et al. 2008), ist das Angebot derzeit marginal.

Bei der Einführung eines Angebotes von Tierschutzfleisch stellt sich für Einzelhändler die Frage, ob die Produkte über die Bedienungs- oder die Selbstbedienungstheke vertrieben werden sollen. Die Platzierung an beiden Theken ist aus Platzgründen und den zu berücksichtigenden Opportunitätskosten i. d. R. nicht möglich (Hübner und Kuhn 2012). Die Ergebnisse in Artikel III.2 zeigen auf, dass Tierschutzfleisch am erfolgversprechendsten an der Bedie- 
nungstheke $\mathrm{zu}$ vertreiben ist, da hier die Zielgruppe im quantitativ größten Umfang erreicht werden kann. Eine entsprechende Erhebung ist auch für andere Nischenprodukte sinnvoll und liefert wichtige Erkenntnisse für eine erfolgversprechende Produktvermarktung. Die Einführung von bspw. laktose- oder glutenfreien Produkten stellt Einzelhändler vor ähnliche Herausforderungen. Der Regalplatz ist begrenzt, weshalb eine Verdopplung der Produktauswahl nicht zu realisieren ist. Zudem entstehen Opportunitätskosten, wenn andere Produkte ausgelistet werden. Diese Herausforderungen zeigen, dass die Einführung von Nischenprodukten aus Marketingsicht solide vorbereitet werden muss. Der geringe Marktanteil der Tierschutzprodukte mit dem Label „Für mehr Tierschutz“ ist auf Grund der Forschungsergebnisse auch z. T. auf die Platzierung in der Selbstbedienungstheke zurückzuführen. Der Handel sollte reagieren und die Produkte über die Bedienungstheke vertreiben, da die Zielgruppe hier besser erreicht werden kann. Da der Wunsch der Verbraucher nach Tierschutzfleisch in verschiedenen Studien nachgewiesen wurde (Harper und Makatouni 2002, Kehlbacher et al. 2012, Lagerkvist und Hess 2011, Schulze et al. 2008), sollten Hersteller, Verarbeiter und Vermarkter das Potenzial nutzen und Tierschutzfleisch und -produkte durch ein ausgereiftes Marketingkonzept etablieren.

\section{Fazit}

Die in den Studien angeführten Limitationen und der weitere Forschungsbedarf zeigen auf, dass die Thematik der Labelling Policies sehr umfassend und komplex ist. Die Dissertation liefert einen wichtigen Beitrag zur Konsumentenforschung. Es werden Ergebnisse vorgestellt, die Potenziale für eine mehrstufige Lebensmittelkennzeichnung ermitteln. Bisher wurde dieses Kennzeichnungstool in der Literatur mit Ausnahme der Nährwertkennzeichnung nicht analysiert, was den Innovationsgrad der Dissertation begründet. Es zeigt sich, dass ein mehrstufiges Kennzeichnungssystem am Lebensmittelmarkt umsetzbar ist und dass der Umsatz mittels gelabelter Produkte erhöht werden kann. Zudem hat ein mehrstufiges Labelsystem Potenzial zur Verbesserung von ethischen Aspekten wie z. B. Tierwohl.

Das Verbraucherverständnis vieler weiterer Kennzeichnungselemente hat bisher in der Literatur kaum Beachtung gefunden. Daher liefert Kapitel 2 wichtige innovatie Ergebnisse für die Konsumentenforschung. Die Herausforderung für Politik und Wirtschaft besteht darin, die Lebensmittelkennzeichnung an das Verbraucherverständnis anzugleichen. Es wurde aufgezeigt, dass zwischen der Produktbeschaffenheit bzw. Prozesseigenschaft und dem Verbraucherverständnis nicht immer Kongruenz besteht. Daher sollten sowohl Hersteller und Produ- 
zenten, aber auch die Gesetzgebung bei der Erstellung und Verwendung von Schlüsselinformationen das Verbraucherverständnis der Produktaufmachung einbeziehen.

Ergänzend geben die abgeleiteten Marketingstrategien für Tierwohlprodukte der Wirtschaft wichtige Impulse, wie ethische (Nischen-)Produkte am besten vermarktet werden können. Im Zusammenhang mit der Wahl eines geeigneten Labellingsystems und der verbrauchergerechten Produktaufmachung können Lebensmittel erfolgreich am Markt etabliert werden.

Letztlich liegt allen Kapiteln die Theorie zu Grunde, dass die kognitive Aufnahmekapazität und Verarbeitungsfähigkeit limitierend sind (Verbeke 2005). Dies beschränkt die Informationsverarbeitung und steht in engem Zusammenhang mit begrenzter Rationalität (bounded rationality) (Simon 1955 und 1959). Der Rationalität bei Einkaufsentscheidungen stehen z. B. Informationsbeschaffungskosten (Zeit der Inforamtionssuche) und Unsicherheit (Informationsasymmetrie) gegenüber. Daher beruhen Verbraucherentscheidungen beim Lebensmitteleinkauf oft auf Heuristiken (Bettman et al. 1998). Diese vereinfachten Entscheidungsregeln können an Schlüsselinformationen wie Label oder Prozesseigenschafen gebunden sein. Basierend auf diesen Theorien wird die Notwendigkeit verstärkt, prägnante Informationen zu geben, um verzerrte Kauf- oder Nichtkaufentscheidungen zu vermeiden.

\section{Ausblick}

Die Einführung eines mehrstufigen Labelsystems kann Unterschiede in Prozess- und Produktstandards transparent kommunizieren. Zugleich würde jedoch auch die Labelflut verstärkt. Das zusätzliche Kennzeichnungselement würde Verpackungen noch überladener wirken lassen. Zudem weisen viele Label auch Jahre nach der Einführung einen sehr geringen Bekanntheitsgrad auf (Buxel 2010, von Meyer-Höfer und Spiller 2012). Die Einführung eines mehrstufigen Labelsystems erfordert daher die Begleitung von einer zielgerichteten Marketingkampagne. Eine langfristige Strategie wäre die Einführung eines zu konzipierenden Dachlabelkonzepts, wie es von Wissenschaftlichen Beiräten für Verbraucher- und Ernährungspolitik sowie Agrarpolitik (2011) vorgeschlagen wird. Verschiedene Kennzeichnungselemente wie z. B. Tierschutz oder Umweltwirkungen können in einem Label getrennt ausgewiesen werden, sodass eine leichte Erkennbarkeit gegeben ist und sich die Suchkosten für Konsumenten reduzieren. Eine praxisgeeignete Lösung wäre die Integration derzeit verwendeter binärer Label. Dies würde zu einer Reduktion der Vielzahl z. T. relativ unbekannter Label und damit auch zur Reduktion der Informationsüberlastung führen. Eine Einbeziehung von Stakeholdern kann zu einer gesteigerten Akzeptanz und damit auch zu einer intensiven Nutzung eines fakultativen, mehrstufigen Labels führen. 
Weiterhin ist anzumerken, dass ein mehrstufiges Kennzeichnungssystem auf Grund der Komplexität und der bewertend wirkenden Stufigkeit kaum durch die Marktteilnehmer selbst zu initiieren und umzusetzen sein wird. Daher sollte der Träger eines mehrstufigen Labelsystems staatlich sein, wie es auch von den Wissenschaftlichen Beiräten für Verbraucher- und Ernährungspolitik sowie Agrarpolitik (2011) empfohlen wird. Ein staatlich getragenes Label kann zudem mit einer Marketingkampagne ähnlich zum Bio-Label einen höheren Bekanntheitsgrad erreichen als einzelne, privatwirtschaftlich getragene Label. Weiterhin ist aus der Literatur bekannt, dass staatlich kontrollierte Label mehr Vertrauen und Glaubwürdigkeit hervorrufen (Sønderkov and Daugberg 2011).

Es ist zu erwarten, dass die Kennzeichnung von Lebensmitteln in den kommenden Jahren ein viel diskutiertes Thema bleiben wird. Nicht zuletzt vor dem Hintergrund, dass die Agrar- und Ernährungsindustrie ein wichtiger Wirtschaftsfaktor in Deutschland ist (BVE 2014, IVV und WZW 2010), sollte daher weitere Forschung - von staatlicher aber auch von privater Seite gefördert werden, die eine verbrauchergerechte Lebensmittelkennzeichnung zum Ziel hat. Eine verbesserte Kennzeichnung, die qualitative Unterschiede deutlich macht, setzt zudem ökonomische Anreize zur Qualitätssteigerung. Die Ernährungsindustrie kann durch eine verständliche Kennzeichnung sowie transparente, nachvollziehbare Kriterien das derzeit geringe Vertrauen in die Lebensmittelindustrie erhöhen (BMEL 2014, Nestlé 2012). Anbieter sollten die Lebensmittelkennzeichnung als ein wertvolles Instrument schätzen, Produktvorteile herauszustellen. Wenn Kunden mit ihrer Produktwahl zufrieden sind und keine Enttäuschung erleben, weil sich ein Produkt anders erweist, als sie es im Supermarkt erwartet haben, führt dies zum Wiederkauf. Zudem kann so die Reputation des Herstellers gestärkt werden.

Gelingt es dem Markt nicht, Vertrauenseigenschaften einheitlich und verständlich zu kommunizieren, sollte der Staat im Akerlof'schen Sinne regulierend eingreifen und Standards festlegen. Die Gesetzgebung ist ebenso gefordert, lebensmittelrechtliche Kennzeichnungsvorschriften an sich verändernde Technologien und Verbraucherbedürfnisse anzupassen. Im Rahmen der Europäisierung ist den Herausforderungen der Lebensmittelkennzeichnung langfristig auf multinationaler Ebene zu begegnen. Labelling Policies müssen auf langfristige Sicht dem gesamteuropäischen Markt gerecht werden. Vorbereitend sind nationale Labelling Policies unabdingbar, die in der Praxis etabliert werden müssen, bevor sie ausgeweitet werden. Jedoch sind Labelling Policies nur dann erfolgreich, wenn die Kennzeichnungstools bekannt und verständlich sind. 


\section{Literatur}

Akerlof, G.A. (1970). The market for "Lemons": qualitative uncertainty and the market mechanism. Quarterly Journal of Economics. 84 (3), 488-500.

Antle, J.M. (2001). Economic analysis of food safety. In: Gardner, B., Rausser, G. (Hrsg.), Handbook of agricultural economics, 1B Amsterdam, S. 1084-1136.

Bettman, J.R., Luce, M.F., Payne, J.W. (1998). Constructive consumer choice processes. Journal of Consumer Research 25 (3), 187-217.

BMEL Bundesministerium für Ernährung und Landwirtschaft (2014). Einkaufs- und Ernährungsverhalten in Deutschland. TNS-Emnid-Umfrage des BMEL. URL: http://www.bmel.de/SharedDocs/Downloads/Umfragen/TNS-Emnid-EinkaufsErnaehrungs VerhaltenInDeutschland.pdf?_blob=publicationFile. Abrufdatum: 20.08.2014.

Buxel, H. (2010). Akzeptanz und Nutzung von Güte- und Qualitätssiegeln auf Lebensmitteln. Fachhochschule Münster. URL: https://www.fh-muenster.de/fb8/downloads/ buxel/10_Studie_Lebensmittelsiegel.pdf. Abrufdatum: 02.02.2015.

BVE (2014). Ernährungsindustrie. URL: file:///C:/Users/rwe/Downloads/bvestatistikbroschuere2014-1.pdf. Abrufdatum: 02.02.2015.

BVE (Hrsg.) (2011). Consumers' Choice '11 - Lebensmittelqualität im Verbraucherfokus: Chancen für Ernährungsindustrie und Handel, Nürnberg.

Canavari, M., Asioli, D., Bendini, A., Cantore, N., Gallina Toschi, T., Spiller, A., Obermowe, T., Buchecker, K., Lohmann, M. (2009). Summary report on sensory-related socioeconomic and sensory science literature about organic food products. URL: http://orgprints.org/17208/2/deliverable_1_2_sensory_literature.pdf. Abrufdatum: 02.02.2015.

Caswell, J.A., Joseph, S. (2008). Consumer demand for quality: major determinant for agricultural and food trade in the future? Journal of International Agricultural Trade and Development 4 (1), 99-116.

Caswell, J.A., Mojduszka, E.M. (1996). Using informational labeling to influence the market for quality and in food products. American Journal of Agricultural Economics 78 (5), 1248-1253.

Caswell, J.A., Padberg, D.I. (1992). Toward a more comprehensive theory of food labels. American Journal of Agricultural Economics. 74 (2), 460-468.

Chandon, P., Wansink, B. (2007). The biasing health halos of fast-food restaurant Health Claims: lower calorie estimates and higher side-dish consumption intentions. Journal of Consumer Research 34 (3), 301-314.

Dabbert, S. (2001). Der Öko-Landbau als Objekt der Politik. Beitrag präsentiert bei der Konferenz: 6. Wissenschaftstagung zum Ökologischen Landbau - Von LeitBildern zu LeitLinien, Freising-Weihenstephan, 06.-08.03.2001. In Reents, H.-J. (Hrsg.) Beiträge zur 6. Wissenschaftstagung zum Ökologischen Landbau, 39-43. Verlag Dr. Köster, Berlin.

Darby, M.R., Karni, E. (1973). Free competition and the optimal amount of fraud. Journal of Law and Economics. 16 (1), 67-88.

Dutra de Barcellos, M., Krystallis, A., Stela de Melo Saab, M., Kügler, J.O., Grunert, K.G. (2011). Investigating the gap between citizens' sustainability attitudes and food purchasing behaviour: empirical evidence from Brazilian pork consumers. International Journal of Consumer Studies 35 (4), 391-402. 
Fernqvist, F., Ekelund, L. (2014). Credence and the effect of consumer liking on food. Food Quality and Preference 32 (Part C), 340-353.

Giesler, M., Veresui, E. (2014). Creating the responsible consumer: moralistic governance regimes and consumer subjectivity. Journal of Consumer Research 41 (3), 840-857.

Gröppel-Klein, A. (2012). Aktuelle Forschungsergebnisse zum Konsumentenverhalten bei Lebensmitteln. In BMELV: Fachtagung Täuschungsschutz bei Lebensmitteln - Erfahrungen - Herausforderungen - Lösungsansätze.

Grunert, K.G (2005). Food quality and safety: consumer perception and demand. European Review of Agricultural Economics 32 (3), 369-391.

Grunert, K.G., Hieke, S., Wills, J. (2014). Sustainability labels on food products: Consumer motivation, understanding and use. Food Policy 44, 177-189.

Harper, G., Makatouni, A. (2002). Consumer perception of organic food production and farm animal welfare. British Food Journal 104 (3), 287-299.

Heinzle, S., Wüstenhagen, R., (2010). Disimproving the European energy label's value for consumers? - Results of a consumer survey. St. Gallen.

Hohmann, G., (1994). Die Verkehrsauffassung im deutschen und europäischen Lebensmittelrecht. P.O.C.-Verlag, Bayreuth.

Hübner, A. H., Kuhn, H. (2012). Retail category management: State-of-the-art review of quantitative research and software applications in assortment and shelf space management. Omega 40 (2), 199-209.

IVV (Frauenhofer-Institut für Verfahrenstechnik und Verpackung) und WZW (TU München Wirtschaftszentrum Weihenstephan) (2010). Studie zum Innovationssektor Lebensmittel und Ernährung. URL: http://www.bmbf.de/pubRD/studie_ernaerungsforschung.pdf. Abrufdatum: 02.02.2015.

Jahn, G., Schramm, M., Spiller, A. (2005). The reliability of certification : quality labels as a consumer policy tool. Journal of Consumer Policy 28(1), 53-73.

Johansson-Stenman, O., Svedsäter, (2012). Self-image and valuation of moral goods: Stated versus actual willingness to pay. Journal of Economic Behavior \& Organization 84 (3), 879-891.

Kehlbacher, A., Bennett, R., Balcombe, K. (2012). Measuring the consumer benefits of improving farm animal welfare to inform welfare labelling. Food Policy 37 (6), 627-633.

Lagerkvist, C.J., Hess, S. (2011). A meta-analysis of consumer willingness to pay for farm animal welfare. European Review of Agricultural Economics 38 (1), 55-78.

Nelson, P. (1970). Information and consumer behaviour. Journal of Political Economy 78 (2), 311-329.

Nestlé (2012). Das is(s)t Qualität. Auszüge aus der Nestlé Studie 2012. URL: http://www.nestle.de/asset-library/documents/verantwortung/nestle\%20studie/executive_ summary_studie_2012.pdf. Abrufdatum: 20.08.2014.

Oakes, M.E., Slotterback, C.S. (2001). What's in a name? A comparison of men's and women's judgements about food names and their nutrient contents. Appetite 36, 29-40.

Palma, M.A., Collart, A.J., Chammoun, C.J. (2014). Information asymmetry in consumer perceptions of quality-differentiated food products. Journal of Consumer Affairs. Article first published online: 27 Nov 2014. DOI: 10.1111/joca.12053 
Rehlender, B. (2012). Die Leitsätze des Deutschen Lebensmittelbuches als wichtige Orientierungshilfe. In BMELV: Fachtagung Täuschungsschutz bei Lebensmitteln - Erfahrungen Herausforderungen - Lösungsansätze.

Rixen, S. (2014). Legitimationsdefizite des Lebensmittelrechts - Zur demokratischen Legitimation der Deutschen Lebensmittelbuch-Kommission. Das Deutsche Verwaltungsblatt 15, 949-958.

Schleyer, A., W. Lorleberg and M. Mergenthalter (2013). Steigerung der landwirtschaftlichen Wertschöpfung durch Produkte aus Weidehaltungssystemen. Final report 30. Fachhochschule Südwestfalen, Soest, Germany. URL: http://www4.fhswf.de/media/downloads/fbaw_1/download_1/lorleberg/Weidemilch_Abschlussbericht_1 31008_final.pdf. Abrufdatum: 17.11.2013.

Schulze, B., Spiller, A., Lemke, D. (2008). Glücksschwein oder arme Sau? Die Einstellung der Verbaucher zur modernen Nutztierhaltung. In Spiller, A., Schulze, B. (Hrsg.). Zukunftsperspektiven der Fleischwirtschaft - Verbraucher, Märkte Geschäftsbeziehungen, S. 465-489. Göttingen, Universitätsverlag Göttingen

Simon, H.A. (1955). A behavioral model of rational choice. The Quarterly Journal of Economics 69 (1), 99-118.

Simon, H.A. (1959). Theories in decision-making in economics and behavioral science. The American Economic Review 49 (3), 253-283.

Simonsons, I., Tversky, A. (1992). Choice in context: Tradeoff contrast and extremeness aversion. Journal of Marketing Research 29 (3), 281-295.

Smith, G.E., Nagle, T.T. (1995). Frames of reference and buyers' perception of price and value California Management Review 38 (1), 98-116.

Sønderkov, K.M., Daugbjerg, C. (2011). The state and consumer confidence in eco-labeling: organic labeling in Denmark, Sweden, The United Kingdom and The United States. Agriculture and Human Value 28 (4), 507-517.

Sprengel, D. (2009). Anbindehaltung bei Milchkühen in Bayern. Landeskuratorium der Erzeugerringe für tierische Veredelung in Bayern e.V., München.

Statistisches Bundesamt (2011). Land- und Forstwirtschaft, Fischerei, Wirtschaftsdünger, Stallhaltung, Weidehaltung Landwirtschaftszählung/Agrarstrukturerhebung 2010. Statistisches Bundesamt Wiesbaden Fachserie 3, Buch 6.

Tversky, A., Simonson, I. (1993). Context-depended differences. Management Science 39 (10), 1179-1189.

Verbeke, W. (2005). Agriculture and the food industry in the information age. European Review of Agricultural Economics 32 (3), 347-368.

Vlaev, I. (2012). How different are real and hypothetical decisions? Overestimation, contrast and assimilation in social interaction. Journal of Economic Psychology 33 (5), 963-972.

von Meyer-Höfer, M., Nitzko, S., Spiller, A. (2013). Expectation Gaps and Halo-Effects in Organic Food Positioning: Characteristics of Organic Food from a Consumer's Point of View. GlobalFood Discussion Papers. No. 25. Universität Göttingen.

von Meyer-Höfer, M., Spiller, A. (2012). Anforderungen an eine nachhaltige Land- und Ernährungswirtschaft : Die Rolle des Konsumenten. KTBL-Schrift 500 Steuerungsinstrumente für eine nachhaltige Land- und Ernährungswirtschaft - Stand und Perspektiven, S. 1-9. 
Wissenschaftliche Beiräte für Verbraucher- und Ernährungspolitik sowie Agrarpolitik des Bundesministeriums für Ernährung, Landwirtschaft und Verbraucherschutz (2011). Politikstrategie Food Labelling. URL: http://www.bmel.de/Shared Docs/Downloads/Ministerium/Beiraete/Verbraucherpolitik/2011_10_PolitikstrategieFood Labelling.pdf?_blob=publicationFile. Abrufdatum: 02.02.2015.

Zühlsdorf, A., Nitzko, S., Spiller, A. (2013). Kennzeichnung und Aufmachung von Lebensmitteln aus Sicht der Verbraucher: Empirische Untersuchungsbefunde, Göttingen. 


\section{Danksagung}

Eine ereignis- und lehrreiche Promotionszeit neigt sich dem Ende zu. An dieser Stelle möchte ich mich bei allen bedanken, die zum Gelingen meiner Promotion beigetragen und mich in dieser Zeit begleitet haben.

Mein besonderer Dank gilt meinem Doktorvater Prof. Dr. Achim Spiller, der mir die Möglichkeit gegeben hat, meinen wissenschaftlichen Forschungsschwerpunkt selbstständig zu wählen und der mir mit seinem Rat immer eine große Hilfe war. Weiterhin geht mein Dank an PD Dr. Gunnar Breustedt für die Übernahme des Zweitgutachtens. An dieser Stelle auch vielen Dank an dich für die Betreuung meiner Abschlussarbeiten an der Universität Kiel, die maßgeblich dazu beigetragen hat, mich für ein Promotionsstudium zu entscheiden. Mein Dank geht ebenso an PD Dr. Thomas Ellrott, der das Prüfungskomitee komplettiert.

Ein weiteres Dankeschön geht an die Agrifood Consulting, insbesondere an Prof. Dr. Julian Voss und Dr. Anke Zühlsdorf, die mir die Möglichkeit gegeben haben, neben der beruflichen Tätigkeit wissenschaftlich zu arbeiten und diese Dissertationsschrift anzufertigen.

Ebenso ein dickes Dankeschön an meine Kollegen im Blauen Turm und bei der Agrifood Consulting, die immer ein offenes Ohr für Probleme hatten. Ein großer Dank geht auch die gute Seele des Lehrstuhls, Petra Geile, für zahlreiches Korrekturlesen und die Unterstützung bei organisatorischen Tätigkeiten. Ein besonderer Dank gilt meinen Koautorinnen, die zum Gelingen der Beiträge in dieser Doktorarbeit durch die hervorragende Zusammenarbeit und den wissenschaftlichen Austausch maßgeblich beigetragen haben.

Des Weiteren geht mein Dank an alle meine Freunde in und außerhalb von Göttingen. Vielen Dank für die vielen aufmunternden Worte, zahlreiche Telefonate und gemeinsam verbrachte Abende und Wochenenden! Ihr habt mir aus jeglichem Motivationstief herausgeholfen.

$\mathrm{Zu}$ besonderem Dank bin ich ebenfalls meinen Eltern verpflichtet. Ihr habt mir meinen Weg geebnet, mich unterstützt und hattet zu jeder Tages- und Nachtzeit gute Ratschläge bei jeglichen Sorgen, Problemen und Ängsten.

Das größte Dankschön gilt dir, Timo. Ohne dich und deine liebevolle, positive Art und deine aufbauenden Worte wäre meine Promotionszeit nicht halb so schön gewesen. 


\section{Erklärungen}

1. Hiermit erkläre ich, dass diese Arbeit weder in gleicher noch in ähnlicher Form bereits anderen Prüfungsbehörden vorgelegen hat.

Weiter erkläre ich, dass ich mich an keiner anderen Hochschule um einen Doktorgrad beworben habe.

Göttingen, den 15. März 2015

(Unterschrift)

2. Hiermit erkläre ich eidesstattlich, dass diese Dissertation selbständig und ohne unerlaubte Hilfe angefertigt wurde.

Göttingen, den 15. März 2015

(Unterschrift) 